\title{
Laser-Interference Surface Preparation for Enhanced Coating Adhesion and Adhesive Joining of Multi-Materials
}

Laser-interference principle, optical setup, and large-scale processing
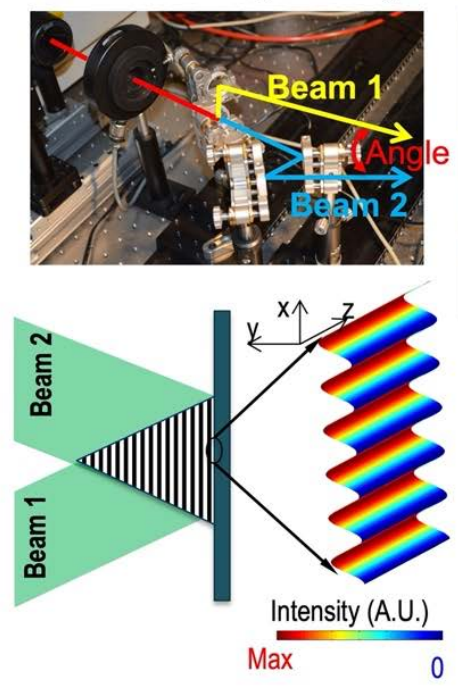

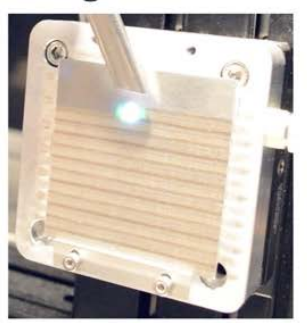

Excellent coating adhesion

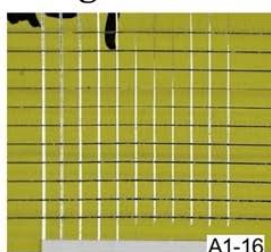

Good corrosion resistance

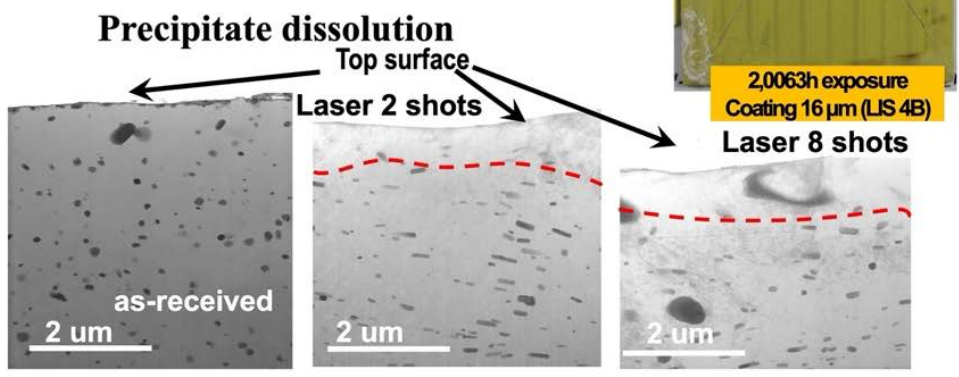

Adrian S. Sabau

Harry Meyer III

Jiheon Jun

Jianlin Li

Donovan Leonard

Approved for public release.

Distribution is unlimited.

September 2021

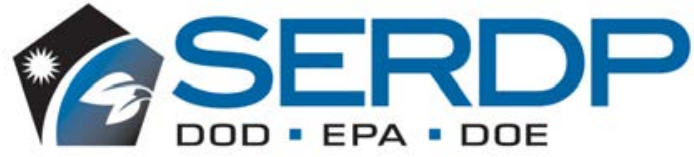




\section{DOCUMENT AVAILABILITY}

Reports produced after January 1, 1996, are generally available free via US Department of Energy (DOE) SciTech Connect.

Website www.osti.gov

Reports produced before January 1, 1996, may be purchased by members of the public from the following source:

National Technical Information Service

5285 Port Royal Road

Springfield, VA 22161

Telephone 703-605-6000 (1-800-553-6847)

TDD 703-487-4639

Fax 703-605-6900

E-mail info@ntis.gov

Website http://classic.ntis.gov/

Reports are available to DOE employees, DOE contractors, Energy Technology Data Exchange representatives, and International Nuclear Information System representatives from the following source:

Office of Scientific and Technical Information

PO Box 62

Oak Ridge, TN 37831

Telephone 865-576-8401

Fax 865-576-5728

E-mail reports@osti.gov

Website https://www.osti.gov/ 
Computational Science and Engineering Division

\title{
FINAL PROJECT REPORT - LASER-INTERFERENCE SURFACE PREPARATION FOR ENHANCED COATING ADHESION AND ADHESIVE JOINING OF MULTI-MATERIALS
}

\author{
Adrian S. Sabau (Lead Principal Investigator) \\ Harry Meyer III \\ Jiheon Jun \\ Jianlin Li \\ Donovan Leonard
}

September 2021

SERDP project WP-2743 “Laser-Interference Surface Preparation for Enhanced Coating Adhesion and Adhesive Joining of Multi-Materials"

Performing organization: Oak Ridge National Laboratory

Prepared by

OAK RIDGE NATIONAL LABORATORY

Oak Ridge, TN 37831-6283

managed by

UT-BATTELLE LLC

for the

US DEPARTMENT OF ENERGY

under contract DE-AC05-00OR22725 


\begin{tabular}{|c|c|c|c|c|c|}
\hline \multicolumn{4}{|c|}{ REPORT DOCUMENTATION PAGE } & \multicolumn{2}{|c|}{$\begin{array}{l}\text { Form Approved } \\
\text { OMB No. 0704-0188 }\end{array}$} \\
\hline \multicolumn{2}{|l|}{$\begin{array}{l}\text { 1. REPORT DATE } \\
09 / 30 / 2021\end{array}$} & \multicolumn{2}{|l|}{$\begin{array}{l}\text { 2. REPORT TYPE } \\
\text { Final research }\end{array}$} & \multicolumn{2}{|c|}{$\begin{array}{l}\text { 3. DATES COVERED (From - To) } \\
\text { March } 2017 \text { - Sept. } 2021\end{array}$} \\
\hline \multirow{3}{*}{\multicolumn{4}{|c|}{$\begin{array}{l}\text { 4. TITLE AND SUBTITLE } \\
\text { LASER-INTERFERENCE SURFACE PREPARATION FOR } \\
\text { ENHANCED COATING ADHESION AND ADHESIVE JOINING OF } \\
\text { MULTI-MATERIALS }\end{array}$}} & \multicolumn{2}{|c|}{$\begin{array}{l}\text { 5a. CONTRACT NUMBER } \\
\text { DE-AC05-00OR22725 }\end{array}$} \\
\hline & & & & \multicolumn{2}{|c|}{ 5b. GRANT NUMBER } \\
\hline & & & & \multicolumn{2}{|c|}{ 5c. PROGRAM ELEMENT NUMBER } \\
\hline \multirow{3}{*}{\multicolumn{4}{|c|}{$\begin{array}{l}\text { 6. AUTHOR(S) Sabau, Adrian, S.; Meyer, Harry, III; Jun, Jiheon; Li, Jianlin } \\
\text { and Leonard, Donovan }\end{array}$}} & \multicolumn{2}{|c|}{$\begin{array}{l}\text { 5d. PROJECT NUMBER } \\
\text { WP-2743 }\end{array}$} \\
\hline & & & & \multicolumn{2}{|c|}{$\begin{array}{l}\text { 5e. TASK NUMBER } \\
\text { N/A }\end{array}$} \\
\hline & & & & \multicolumn{2}{|c|}{$\begin{array}{l}\text { 5f. WORK UNIT NUMBER } \\
\text { N/A }\end{array}$} \\
\hline \multicolumn{4}{|c|}{$\begin{array}{l}\text { 7. PERFORMING ORGANIZATION NAME(S) AND ADDRESS(ES) } \\
\begin{array}{l}\text { OAdrian S. Sabau RIDGE NATIONAL LABORATORY } \\
\text { Oak Ridge, TN } 37831-6283\end{array}\end{array}$} & \multicolumn{2}{|c|}{$\begin{array}{l}\text { 8. PERFORMING ORGANIZATION } \\
\text { REPORT } \\
\text { ORNËLISPR-2021/2257 }\end{array}$} \\
\hline \multirow{2}{*}{\multicolumn{4}{|c|}{$\begin{array}{l}\text { 9. SPONSORING / MONITORING AGENCY NAME(S) AND ADDRESS(ES) } \\
\begin{array}{ll}\text { Robin Nissan } & \text { Strategic Environmental Research and Development } \\
& 4800 \text { Mark Center Drive, Suite 17D03, Alexandria, VA } \\
& 22350\end{array}\end{array}$}} & \multicolumn{2}{|c|}{$\begin{array}{l}\text { 10. SPONSOR/MONITOR'S } \\
\text { ACRONYM(S) } \\
\text { SERDP }\end{array}$} \\
\hline & & & & \multicolumn{2}{|c|}{$\begin{array}{l}\text { 11. SPONSOR/MONITOR'S } \\
\text { REPORT NUMBER(S) } \\
\text { WP- } 7743\end{array}$} \\
\hline \multicolumn{6}{|c|}{$\begin{array}{l}\text { 12. DISTRIBUTION / AVAILABILITY STATEMENT } \\
\text { Approved for public release; distribution is unlimited }\end{array}$} \\
\hline \multicolumn{6}{|c|}{$\begin{array}{l}\text { 13. SUPPLEMENTARY NOTES } \\
N / A\end{array}$} \\
\hline \multicolumn{6}{|c|}{$\begin{array}{l}\text { 14. ABSTRACT } \\
\text { This report investigates a laser-interference surface treatment as a non-contact, i.e., without major solid/liquid } \\
\text { medium application or abrasion, and non-chemical surface preparation method for aerospace coating systems. It } \\
\text { was proposed to use the laser-interference technique to structure surfaces of Al and/or Ti, creating "rough" surfaces } \\
\text { with pre-engineered series of ridges and valleys at submicron scale. The science goal of this study was to develop } \\
\text { an understanding of the surface microstructure, topology, and physical mechanisms that would improve adhesion } \\
\text { and corrosion protection of Al2024-T3. Microstructure analysis indicates that the laser-interference structuring (LIS) } \\
\text { was found to reduce the formation of CuMn-rich precipitates in Al } 2024-T 3 \text { over a } 500-800 \mathrm{~nm} \text { depth from top } \\
\text { surface. The X-cut and cross-hatch coating adhesion ratings indicate that the LIS specimens meet the performance } \\
\text { requirements in the coating adhesion specifications by having a higher or identical ranking to those specimens } \\
\text { prepared with current state-of-the-art chemical conversion or sulfuric acid anodizing. After the ASTM B117 } \\
\text { corrosion exposure, it was found that the laser processed specimens exhibited only few blisters. It was found } \\
\text { that the corrosion damage was minimized at a laser rastering speed of } 4 \mathrm{~mm} / \mathrm{s} \text {, for which only } 33 \% \text { of specimens } \\
\text { developed very minor corrosion damage. The ASTM D1654 creepage ratings, used to evaluatecorrosion } \\
\text { damage along the scribe lines, were found to be at least nine for all coated panels. These results indicate that } \\
\text { the laser-interference technique with the additional acetone wiping has the potential to be further developed as } \\
\text { a minor chemical surface preparation technique for chromate-containing epoxy primers coatings. }\end{array}$} \\
\hline \multicolumn{6}{|c|}{$\begin{array}{l}\text { 15. SUBJECT TERMS } \\
\text { corrosion, laser, interference, surface treatment, aluminum, primer, precipitates, dissolution }\end{array}$} \\
\hline \multicolumn{3}{|c|}{ 16. SECURITY CLASSIFICATION OF: } & $\begin{array}{l}\text { 17. LIMITATION } \\
\text { OF ABSTRACT }\end{array}$ & \multirow{2}{*}{$\begin{array}{l}\text { 18. NUMBER } \\
\text { OF PAGES } \\
118\end{array}$} & $\begin{array}{l}\text { 19a. NAME OF } \\
\text { RESPONSIBLE } \\
\text { PERSON } \\
\text { Adrian Sabau }\end{array}$ \\
\hline $\begin{array}{r}\text { a. REPORT } \\
\text { U }\end{array}$ & $\begin{array}{l}\text { b. ABSTRACT } \\
U\end{array}$ & $\begin{array}{l}\text { c. THIS PAGE } \\
\text { U }\end{array}$ & UU & & $\begin{array}{l}\text { 19b. TELEPHONE } \\
\text { NUMBER } \\
865241-5145\end{array}$ \\
\hline
\end{tabular}




\section{CONTENTS}

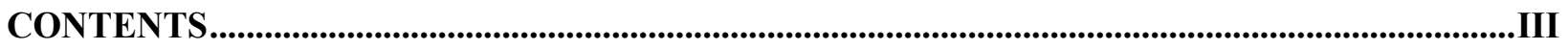

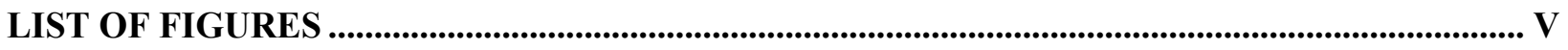

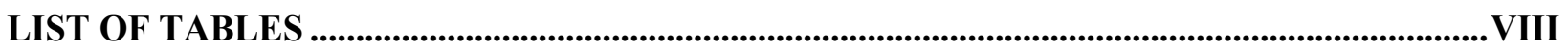

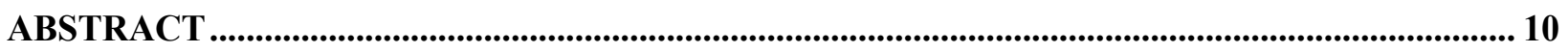

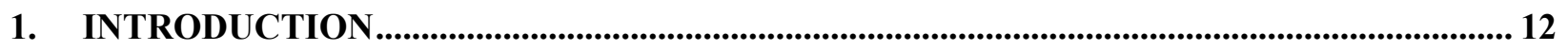

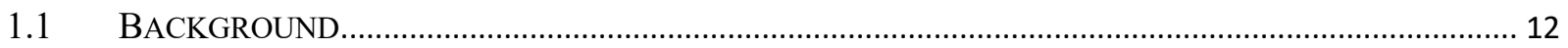

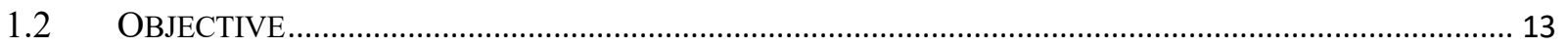

2. MATERIALS, METHODS, AND TECHNICAL APPROACH ............................................ 15

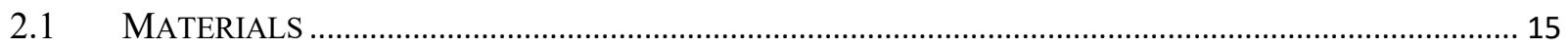

2.1 INTRODUCTION OF LASER-INTERFERENCE STRUCTURING ........................................................... 16

2.2 PHYSICAL PHENOMENA FOR LASER-INTERFERENCE STRUCTURING OF METALS .......................... 17

2.3 QUALITY OF LASER-INTERFERENCE STRUCTURING OF METALS ……........................................ 19

\section{PROCESSING EQUIPMENT AND EXPERIMENTAL PROCEDURES FOR SURFACE}

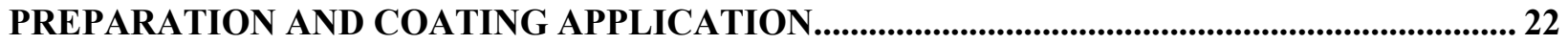

3.1 LASER EQUIPMENT AND OPTICAL SETUP FOR LASER-INTERFERENCE STRUCTURING ................... 22

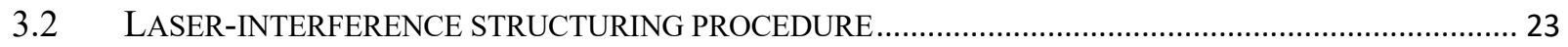

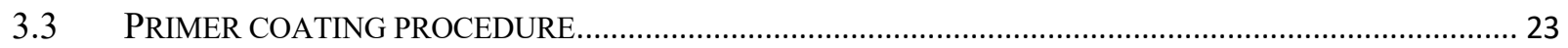

4. MATERIALS CHARACTERIZATION EQUIPMENT AND PROCEDURES ........................ 26

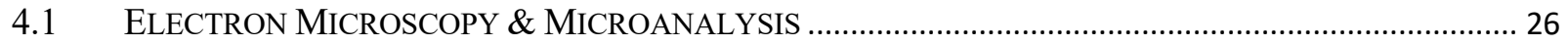

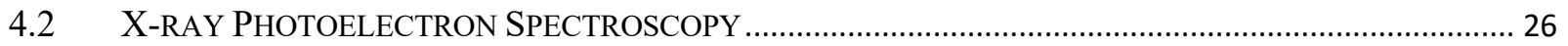

4.3 PROFILOMETRY AND ROUGHNESS ANALYSIS PROCEDURES ............................................................ 27

4.4 COATING ADHESION TEST PROCEDURES AND PROCESS VARIABLES ................................................ 28

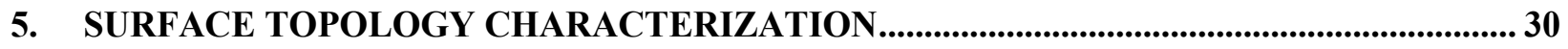

5.1 SURFACE MORPHOLOGY OF AL 5182 OBTAINED BY SPOT-BY-SPOT LASER-INTERFERENCE

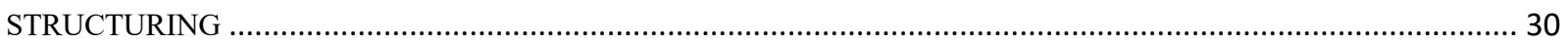

5.2 SURFACE MORPHOLOGY OF AL2024-T3 OBTAINED BY SPOT-BY-SPOT LASER-INTERFERENCE

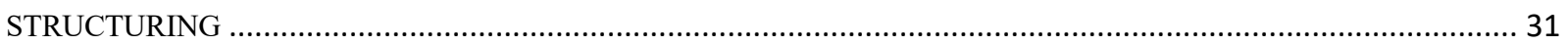

5.3 PROFILOMETRY OF AL2024-T3 SURFACES OBTAINED BY SPOT-BY-SPOT LASER-INTERFERENCE

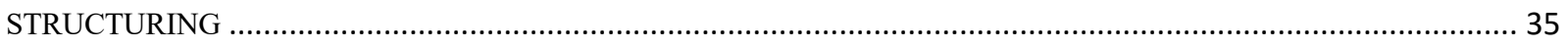

5.4 PROFILOMETRY OF AL2024-T3 SURFACES OBTAINED BY RASTER LASER-INTERFERENCE

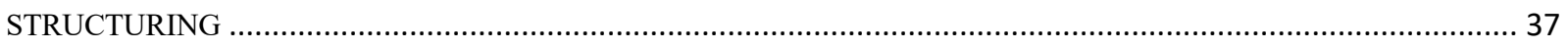

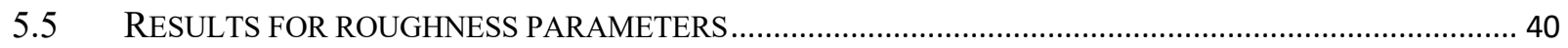

5.6 RESULTS FOR SURFACE AREA OF LASER STRUCTURED SPECIMENS............................................ 41

5.7 VERTICAL SUB-SURFACE MiCROSTRUCTURE OF AL2024-T3 OBTAINED BY SPOT-BY-SPOT

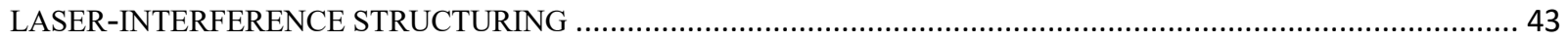

5.8 ELEMENTAL MAPS IN SUBSURFACE CROSS-SECTIONS OF AL 2024 OBTAINED BY SPOT-BY-SPOT

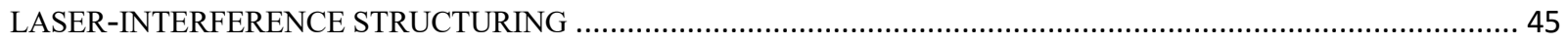

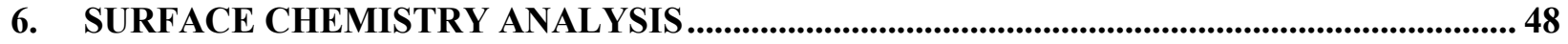

6.1 XPS SURFACE ANALYSIS OF AA 2024-T3 SURFACES: AS-RECEIVED, UNPROCESSED................ 48 
6.2 XPS SURFACE ANALYSIS OF AA 2024-T3 SURFACES LASER-INTERFERENCE PROCESSED

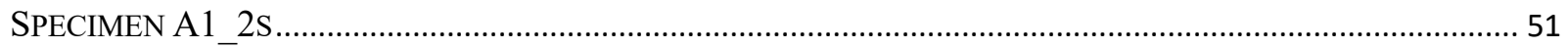

6.3 XPS SURFACE ANALYSIS OF AA 2024-T3 SURFACES LASER PROCESSED SPECIMEN A1_8S .... 54

6.4 EFFECT OF LASER BEAM ANGLE ON SURFACE COMPOSITION ................................................. 59

7. WETTING BEHAVIOR AND SURFACE ENERGIES OF SPOT-BY-SPOT LASER-

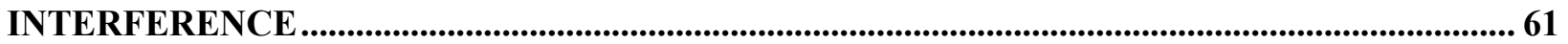

7.1 EXPERIMENTAL PROCEDURE FOR CONTACT ANGLE MEASUREMENT …........................................ 61

7.2 CONTACT ANGLE MEASUREMENTS FOR SPOT-BY-SPOT LASER INTERFERENCE PROCESSED

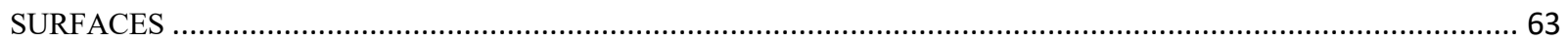

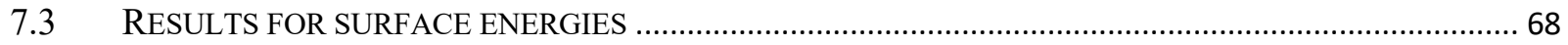

8. RESULTS FOR COATING ADHESION TESTING OF RASTER SPECIMENS.................... 72

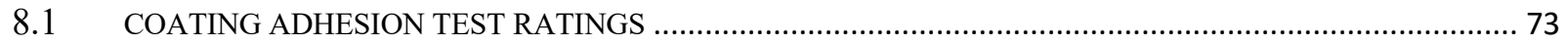

8.2 DISCUSSION OF COATING ADHESION TESTING RESULTS ............................................................. 79

8.3 EFFECT OF OPEN-TIME AND COATING THICKNESS EFFECT ON ADHESION RATING......................... 82

9. LASER TREATMENT FOR CORROSION PROTECTION..................................................... 84

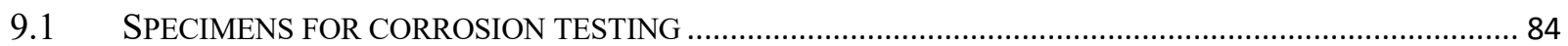

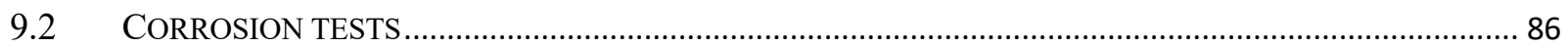

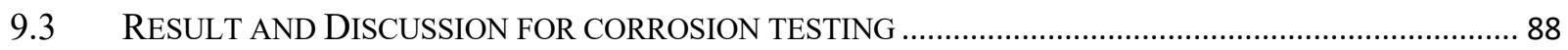

9.3.1 Electrochemical measurement results for uncoated specimens ....................................... 88

9.3.2 Salt spray test results for batch A of coated specimens ................................................... 91

9.3.3 Salt spray test results for batch B of coated specimens ..................................................... 94

9.3.4 Results for roughness parameters: corrosion paper...................................................... 99

9.3.5 Chemical analysis of water used in salt spray tests and evolution of corrosion damage..... 101

9.3.6 X-ray photoelectron spectroscopy of blister regions ..................................................... 102

10. FATIGUE TESTING NEEDED FOR DEMONSTRATION/IMPLEMENTATION ........... 105

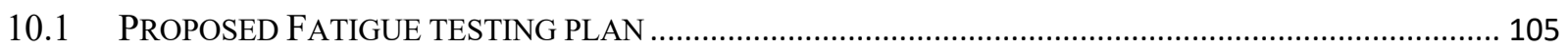

11. CONCLUSIONS AND IMPLICATIONS FOR FUTURE RESEARCH ............................ 107

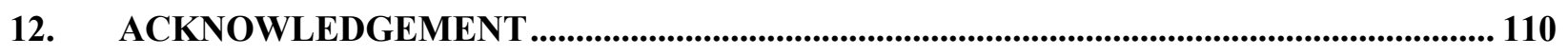

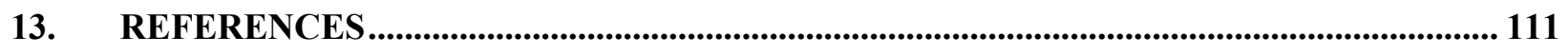

14. APPENDIX A. LIST OF SCIENTIFIC/TECHNICAL PUBLICATIONS ........................... 115 


\section{LIST OF FIGURES}

Figure 1. Schematic of the setup of laser interference system.

Figure 2. Schematic of laser-interference induced structuring patterns as a function of laser

fluence $(\mathrm{F})$.

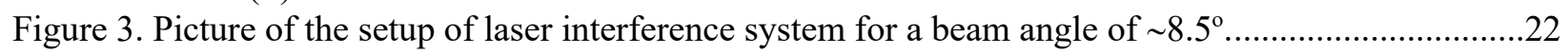

Figure 4. Optical images of as-received Al 5182......

Figure 5. Optical micrograph of laser-interference structured AA5128 surface with a spot size of $4 \mathrm{~mm}$, laser fluence of $1.05 \mathrm{~J} / \mathrm{cm}^{2}$ and the following pulses per spot: (a) 1, (b) 2, and (c) $6 \ldots \ldots \ldots . . .31$

Figure 6. SEM micrograph showing distribution of voids (black, rounded shapes) and precipitates (white, irregular shapes) in vertical cross-section near the top surface.

Figure 7. Top view FESEM images for the as-received condition and four laser-structured conditions at a spatial period of $1.7 \mathrm{~m}$ with $2,4,6$, and 8 laser shots/spot (A0, A1_2s, A1_4s, A1_6s, and A1_8s).

Figure 8. Top view FESEM images for laser-structured specimens at a spatial period of $\sim 0.6 \mathrm{~m}$ with 2, 4, 6, and 8 laser shots/spot (A2_2s, A2 4s, A2_6s, and A2 8s).....

Figure 9. Surface profiles for: (a) as-received specimen (A0) and (b, c) 8 shots/spot specimen A1_8s at a spatial period of $\sim 1.7 \mathrm{~m}$.

Figure 10. Surface profiles at a laser fluence of $F 1=1.238 \mathrm{~J} / \mathrm{cm}^{2}$ per pulse for $(\mathrm{a}, \mathrm{b})$ the asreceived specimens and in typical centerlines of a laser scan for raster speeds of: (c) 2 $\mathrm{mm} / \mathrm{s}$, (d) $4 \mathrm{~mm} / \mathrm{s}$, (e) $6 \mathrm{~mm} / \mathrm{s}$, and (f) $8 \mathrm{~mm} / \mathrm{s}$.

Figure 11. Optical micrographs acquired by the profilometer. The corresponding location of the quarter area to the profilometry images shown in Figure 10 is indicated in parenthesis: (a, b) the as-received specimens (TL, BL) and in the typical centerline of a laser scan for raster speeds of: (c) $2 \mathrm{~mm} / \mathrm{s}$ (BL), (d) $4 \mathrm{~mm} / \mathrm{s}$ (TR), (e) $6 \mathrm{~mm} / \mathrm{s}$ (BL), and (f) $8 \mathrm{~mm} / \mathrm{s}$ (TL). Laser fluence was $F_{1}=1.238 \mathrm{~J} / \mathrm{cm}^{2}$ per pulse.

Figure 12. Schematic of: (a) LIS surface topology and (b) simplified surface profile.

Figure 13. STEM micrographs showing distribution of precipitates (black or dark gray colors) in vertical cross-sections near the top surface for A12024-T3 specimens: (a) as-received and laser-interference processed with (b) 2 shots-per-spot (A1_2s) and (c) 8 shots-per-spot (A1_8s).

Figure 14. Maps for HAADF STEM image for elements $\mathrm{Al}, \mathrm{Cu}, \mathrm{Fe}, \mathrm{Mg}, \mathrm{Mn}$, and $\mathrm{O}$. Data for the vertical cross-section as-received specimen (without any laser processing).

Figure 15. Maps for HAADF STEM image for elements $\mathrm{Al}, \mathrm{Cu}, \mathrm{Fe}, \mathrm{Mg}, \mathrm{Mn}$, and $\mathrm{O}$. Data for the vertical cross-section of the laser-interference processed specimen A1_8s with 8 shots per spot.

Figure 16. Ar-ion depth profile for AA2024 baseline specimen (A0) (without any laser processing, the as-received condition: $(\mathrm{a}, \mathrm{b}) \mathrm{C} 1 \mathrm{~s}, \mathrm{O} 1 \mathrm{~s}, \mathrm{Al} 2 \mathrm{p}$ (peak fit to give both Aloxide and $\mathrm{Al}-\mathrm{metal}), \mathrm{Mg}$, and the $\mathrm{Cu} 2 \mathrm{p}$ signal (multiplied by 5 ) on a scale of $0-100$ at. $\%$ and (c) $\mathrm{Mn} 2 \mathrm{p}, \mathrm{Na} 1 \mathrm{~s}$, and $\mathrm{Cl} 2 \mathrm{p}$ profiles.

Figure 17. Ar-ion depth profile for A AA2024 laser treated material with 2-shots/spot (A1_2s): (a, b) C 1s, O 1s, Al 2p (peak fit to give both Al-oxide and Al-metal), $\mathrm{Mg}$, and the $\overline{\mathrm{Cu}} 2 \mathrm{p}$ signal (multiplied by 5) and (c) $\mathrm{Mn} 2 \mathrm{p}, \mathrm{Na} 1 \mathrm{~s}$, and $\mathrm{Cl} 2 \mathrm{p}$ profiles.

Figure 18. Ar-ion depth profile for A AA2024 laser treated material with 8-shots/spot (A1_8s): (a, b) $\mathrm{C} 1 \mathrm{~s}, \mathrm{O} 1 \mathrm{~s}, \mathrm{Al} 2 \mathrm{p}$ (peak fit to give both Al-oxide and Al-metal), $\mathrm{Mg}$, and the $\overline{\mathrm{Cu}} 2 \mathrm{p}$ signal (multiplied by 5) and (c) $\mathrm{Mn} 2 \mathrm{p}, \mathrm{Na} 1 \mathrm{~s}$, and $\mathrm{Cl} 2 \mathrm{p}$ profiles ....

Figure 19. Al-oxide depth profiles (at. \%) for baseline and laser-interference processed specimens A1_2s and A1_8s. 
Figure 20. Depth profiles for Al-oxide (at. \%) for laser-interference structured specimens with laser-beam angles of $12^{\circ}, 36^{\circ}$ and $72^{\circ}$ for (a) 2 shots/spot (specimens A1_2s, A3_2s, and A2_2s) and (b) 8 shots/spot (specimens A1_8s, A3_8s, and A2_8s).

Figure 21. Examples of contact angle measurement on spot-by-spot treated samples (a) laser spot center locations, (b) 2 spot overlap region, and (c) 3 spot overlap region.

Figure 22. Images of a typical liquid drop (left) and a profile-based contact angle measurement (right).

Figure 23. Contact angle for baseline specimens and in the laser-spot center for specimens processed with beam angle of $72^{\circ}$.

Figure 24. Contact angle for laser-spot center for specimens processed with beam angle of $72^{\circ}$ : (a) dual-overlap and (b) tri-overlap regions.

Figure 25. Deviation [\%] of contact angles: (a) dual-overlap regions and (b) tri-overlap regions with respect to the laser spot center (beam angle of $72^{\circ}$ ).

Figure 26. Contact angle in the laser-spot center for specimens processed with beam angles of: (a) $12^{\circ}$ and (b) $36^{\circ}$......

Figure 27. Pictures of X-cut tested laser-structured Al panels at rastering speeds of (a-c) $4 \mathrm{~mm} / \mathrm{s}$ and (d) $10 \mathrm{~mm} / \mathrm{s}$ at a laser fluence of $F_{1}=1.238 \mathrm{~J} / \mathrm{cm}^{2}$ per pulse. Coating thickness and $X$ cut test score according to ASTM D3359, section 7, are indicated for each figure.

Figure 28. Pictures of X-cut tested Al panels prepared with (a-c) CCC and (d) SAA treatments. Coating thickness and X-cut test score according to ASTM D3359, section 7, are indicated for each figure.

Figure 29. Pictures of cross-hatch tested laser-structured Al panels, test scores (ASTM D3359, section 12), and coating thickness at rastering speed of (a-c) $6 \mathrm{~mm} \cdot \mathrm{s}^{-1}$ and (d-f) $8 \mathrm{~mm} \cdot \mathrm{s}^{-1}$ at a laser fluence of $F_{1}=1.782 \mathrm{~J} / \mathrm{cm} 2$ per pulse. Grid size is $1 \mathrm{~mm} \times 1 \mathrm{~mm}$.

Figure 30. Pictures of cross-hatch tested Al panels test scores (ASTM D3359, section 12), and coating thickness for $(a, b) C C C$ and $(c, e)$ SAA treatments. Grid size is $1 \mathrm{~mm} \times 1 \mathrm{~mm}$.

Figure 31. Histograms for coating adhesion rating for LIS, CCC, and SAA coated panels for the: (a) X-cut and (b) cross-hatch tests. The solid symbols and empty symbols indicate data obtained at fluences $F_{1}$ of 1.238 and $1.782 \mathrm{~J} / \mathrm{cm}^{2}$ per pulse, respectively.

Figure 32. Average adhesion ratings from (a) X-cut and (b) cross-hatch tests for different coating thickness for laser-structured at a laser fluence of $\mathrm{F} 1=1.238 \mathrm{~J} / \mathrm{cm} 2$ per pulse, CCC, and SAA panels.

Figure 33. Laser-structured surfaces of Al 2024 panels with different raster speeds and beam spot sizes: (a) $8 \mathrm{~mm} / \mathrm{s}$ with $\varnothing=5 \mathrm{~mm}$, (b) $4 \mathrm{~mm} / \mathrm{s}$ with $\varnothing=5 \mathrm{~mm}$, (c) $10 \mathrm{~mm} / \mathrm{s}$ with $\varnothing=6 \mathrm{~mm}$

Figure 34. Potentiodynamic curves of (a) a SAA specimen and (b) ABR, CCC, and two LIS specimens at a fluence of $F 1=1.782 \mathrm{~J} / \mathrm{cm}^{2}$ per pulse (LIS 4A and LIS $10 \mathrm{~A}$ ) obtained with a working electrode area of $14.8 \mathrm{~cm}^{2}$. Zero current and breakdown potentials are denoted as $E_{z c}$ and $E_{b}$, respectively. For the SAA specimen, a sharp current increase is considered to indicate a local insulation failure.

Figure 35. Comparison of (a) $E_{z c}$ and $E_{b}$ values and (b) $i_{c-50}$ values for ABR, CCC, and two LIS specimens at a fluence of $F_{1}=1.782 \mathrm{~J} / \mathrm{cm}^{2}$ per pulse (LIS 4A and LIS 10A).

Figure 36. Potentiodynamic (a) anodic curves and (b) cathodic curves of ABR, CCC, and two LIS specimens at a fluence of $F_{l}=1.782 \mathrm{~J} / \mathrm{cm}^{2}$ per pulse (LIS4A and LIS10A) obtained with a working electrode area of $0.49 \mathrm{~cm}^{2}$.

Figure 37. Pictures of coated panels with $\mathrm{CCC}(\mathrm{a}, \mathrm{b})$ and SAA $(\mathrm{c}, \mathrm{d})$ pre-treatments indicating minor corrosion damage after $500 \mathrm{~h}$ or $1000 \mathrm{~h}$ exposures.

Figure 38. Pictures of coated LIS test panels indicating minor or no corrosion damage along the scribe line and one blister after $500 \mathrm{~h}$ exposure for the conditions: (a) LIS 4A, (b) LIS6A, and (c) LIS10A. LIS_A batch was processed at a fluence of $F_{1}=1.782 \mathrm{~J} / \mathrm{cm}^{2}$ with no additional wiping after LIS. 
Figure 39. Pictures of selected LIS test panels with the most corrosion damage after $1000 \mathrm{~h}$ exposure for conditions: (a) LIS 4A, (b) LIS 6A, and (c) LIS 10A. LIS A batch was processed at a fluence of $F_{1}=1.782 \mathrm{~J} / \mathrm{cm}^{2}$ with no additional wiping after LIS.

Figure 40. Evolution of minor blister growth during ASTM B117 salt-spray test for the same panel $(5.08 \mathrm{~cm} \times 7.62 \mathrm{~cm})$ with processing condition LIS 4A and coating thickness 15 $\mathrm{m}$.

Figure 41. Evolution of minor blister growth during ASTM B117 salt-spray test for the same panel $(5.08 \times 7.62 \mathrm{~cm})$ with processing condition LIS2A and coating thickness $21 \mathrm{~m}$.

Figure 42. Pictures of selected LIS test panels showing no corrosion damage after $409 \mathrm{~h}$ exposure for the conditions: (a) LIS 2B, (b) LIS 4B, (c) LIS 6B, and (d) LIS 8B. The second batch was processed at a fluence of $F_{1}=1.238 \mathrm{~J} / \mathrm{cm}^{2}$ and acetone wiped right after LIS.

Figure 43. Pictures of selected LIS test panels with the most corrosion damage after $2063 \mathrm{~h}$ exposure for the conditions: (a) LIS2B, (b) LIS4B, (c) LIS6B, and (d) LIS8B. The second batch was processed at a fluence of $\mathrm{F} 1=1.238 \mathrm{~J} / \mathrm{cm}^{2}$ and acetone wiped right after LIS. The initial appearance of blisters at the off-time inspection is presented in Figure 45 for each coated LIS panel in (a-d).

Figure 44. Pictures of selected LIS test panels with the least corrosion damage after $2063 \mathrm{~h}$ exposure for the conditions: (a) LIS2B, (b) LIS4B, (c) LIS6B, and (d) LIS8B. The second batch was processed at a fluence of $\mathrm{F} 1=1.238 \mathrm{~J} / \mathrm{cm}^{2}$ and acetone wiped right after LIS.

Figure 45. Evolution of the initial blister during ASTM B117 salt-spray test for the coated panels $\left(5.08 \times 7.62 \mathrm{~cm}^{2}\right)(\mathrm{a}-\mathrm{d})$ in this figure correspond to (a-d) in Figure 43.

Figure 46 . Surface profiles at a laser fluence of $F 1=1.782 \mathrm{~J} / \mathrm{cm} 2$ per pulse for $(a, b)$ the as-
received specimens and in typical centerlines of a laser scan for raster speeds of: (c) 4 $\mathrm{mm} / \mathrm{s}$, (d) $6 \mathrm{~mm} / \mathrm{s}$, (e) $8 \mathrm{~mm} / \mathrm{s}$, and (f) $10 \mathrm{~mm} / \mathrm{s}$. 


\section{LIST OF TABLES}

Table 1. Features and differences of proposed technique from conventional laser ablation techniques

Table 2. Survey of structure depth at various periodicities and fluences for one-pulse laser-

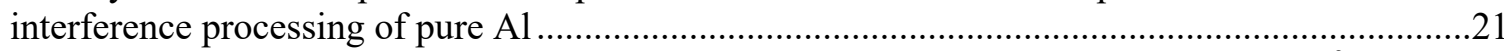

Table 3. Energy metrics for several raster speeds for laser fluences $F_{1}$ of 1.782 and $1.238 \mathrm{~J} / \mathrm{cm}^{2}$ and identification of specimen process conditions (process ID) for corrosion testing ..................23

Table 4. Surface roughness for specimen A1_8s at a spatial period of $\sim 1.7 \mathrm{~m}$.....................................37

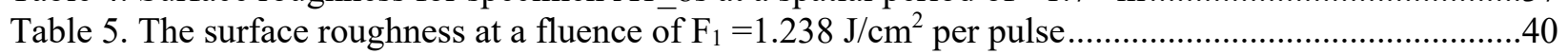

Table 6. The percentage increase in surface roughness metrics with respect to those for the asreceived condition at a fluence of $\mathrm{F} 1=1.238 \mathrm{~J} / \mathrm{cm}^{2}$ per pulse

Table 7. Estimated LIS height, h, and roughness factor, $r L I S$, for several raster speeds .........................43

Table 8. Overall composition (at.\%) in three areas for the Al-2024 baseline specimen (A0), without any laser processing, in the as-received condition...

Table 9. Surface composition (at.\%), without any Ar+ etch, for the laser processed specimen A1_2s (2-shots/spot, laser beam angle of $\left.12^{\circ}\right)$.........................................................................

Table 10. Surface composition (at.\%), without any Ar+ etch, for the laser processed specimen A1_8s $\left(8\right.$-shots/spot, laser beam angle of $\left.12^{\circ}\right)$..........................................................................56

Table 11. Surface Composition (at.\%), without any Ar+ etch, for 2 -shot/spot and 8-shot/spot specimens processed with all three beam angle considered ......................................................59

Table 12. Laser processing conditions for spot-by-spot for contact angle measurements.........................61

Table 13. Surface tension of liquids used for contact angle measurements ...........................................62

Table 14. Mean contact angle $\left[^{\circ}\right]$ and its standard deviation $\left[{ }^{\circ}\right]$ for the two baseline alloys .....................64

Table 15. Contact angle $\left[^{\circ}\right]$ its standard deviation $\left[^{\circ}\right]$ for A1 samples, LIS with $12^{\circ}$ laser beam angle

Table 16. Contact angle $\left[^{\circ}\right]$ its standard deviation $\left[^{\circ}\right]$ for A2 samples, LIS with $72^{\circ}$ laser beam angle.

Table 17. Contact angle $\left[{ }^{\circ}\right]$ its standard deviation $\left[{ }^{\circ}\right]$ for A3 samples, LIS with $36^{\circ}$ laser beam angle

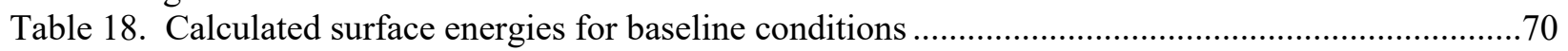

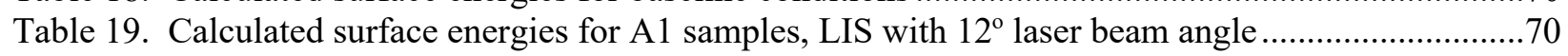

Table 20. Calculated surface energies for A2 samples, LIS with $72^{\circ}$ laser beam angle ...........................70

Table 21. Calculated surface energies of A3 samples, LIS with $36^{\circ}$ laser beam angle .............................72

Table 22. Selection of process parameters for three coating adhesion analyses conducted .......................73

Table 23. Statistical results from Student t-Tests for individual pairs (LIS-LIS) of cross-hatch ratings at fluences of $1.78 \mathrm{~J} / \mathrm{cm} 2$ and $1.24 \mathrm{~J} / \mathrm{cm} 2$ at each rastering speed.

Table 24. Statistical results from Student t-Tests for individual pairs (SAA-LIS) of cross-hatch ratings. LIS data was at fluences of $1.78 \mathrm{~J} / \mathrm{cm} 2$

Table 25. Average, minimum, and lower confidence limit adhesion ratings from X-cut and crosshatch tests for LIS, CCC, and SAA panels coated with CA7233 primer ....

Table 26. The average cross-hatch rating for both $4 \mathrm{~mm} / \mathrm{s}$ and $6 \mathrm{~mm} / \mathrm{s}$ laser raster speeds at two fluences and several open times

Table 27. Energy metrics for several raster speeds for laser fluences $F_{1}$ of 1.782 and $1.238 \mathrm{~J} / \mathrm{cm}^{2}$ and identification of specimen process conditions (process ID).

Table 28. Number of panels per each condition for the ASTM B117 salt spray exposure test.

Table 29. Blister initiation time $\left(\mathrm{t}_{\mathrm{I}}\right)$ for the coated panels in batch B with the percentage of blistered panels per each LIS condition. Refer to Table 27 for the details of LIS conditions

Table 30. The surface roughness at a fluence of $F_{1}=1.238 \mathrm{~J} / \mathrm{cm}^{2}$ per pulse 
Table 31. Chemical analysis on the tap water used in salt spray tests .......

Table 32. Surface composition (at.\%) nominal coating, back-surface of the blister coating, and metal surface below the blister area.

Table 33. Fatigue testing options and costs. 


\begin{abstract}
Introduction: Aluminum-skinned aircraft are typically chemical conversion coated or anodized prior to application of coating systems or adhesive bonding. The environmental risks and costs of managing millions of gallons of spent chemical processing solutions, which often contain hexavalent chromium, other hazardous air pollutants, and/or cyanide as hazardous waste, need to be reduced.
\end{abstract}

The main objective of this project is to demonstrate the use of a one-step process at ambient conditions based on laser-interference structuring (LIS) techniques to replace the chemical conversion coating or anodization prior to the application of coating systems or adhesive bonding for $\mathrm{Al}$ and Ti aircraft components. The science goal of this study was to develop an understanding of the effects of laserinterference structuring on the surface microstructure, topology, and physical mechanisms that would improve adhesion and corrosion protection of A12024-T3.

The technical approach was based on the use of the laser-interference structuring technique as a noncontact, i.e., without major solid/liquid medium application or abrasion, and non-chemical surface preparation method for aerospace coating systems. Specifically this project investigated the effect of laser-interference structuring on: (a) enhancement of coating/paint adhesion and (b) corrosion resistance of the coated or bonded substrate. The laser-interference technique was used to structure surfaces of Al and/or Ti, creating periodic "rough" surfaces with pre-engineered series of ridges and valleys at submicron scale. The laser interference power profile is created by splitting the beam and guiding those beams to the sample surface by overlapping each other with defined angles to each other.

Microstructure analysis indicates that the laser-interference structuring (LIS) was found to reduce the formation of CuMn-rich precipitates in Al 2024-T3 over a 500-800 nm depth from top surface. The precipitate dissolution is expected to lead to an increase in corrosion protection of the laser-interference treated surface as the localized corrosion would be reduced. The ASTM D3359 X-cut and cross-hatch coating adhesion ratings indicate that the LIS specimens meet the performance requirements in the coating adhesion specifications by having a higher or identical ranking to those specimens prepared with current state-of-the-art chemical conversion or sulfuric acid anodizing. After the ASTM B117 corrosion exposure, it was found that the laser processed specimens exhibited only few blisters. It was found that the corrosion damage was minimized at a laser rastering speed of $4 \mathrm{~mm} / \mathrm{s}$, for which only $33 \%$ of specimens developed very minor corrosion damage. The ASTM D1654 creepage ratings, used to evaluate corrosion damage along the scribe lines, were found to be at least nine for all coated panels. 
These results indicate that the laser-interference technique with the additional acetone wiping has the potential to be further developed as a minor chemical surface preparation technique for chromate-containing epoxy primers coatings.

Benefits: The proposed single-step and non-chemical laser-interference processing has the potential to drastically reduce the environmental impact of chemical surface treatments used in the manufacture and maintenance of DoD weapons systems. Moreover, the environmental risks and costs of managing millions of gallons of spent chemical processing solutions as hazardous waste also will be greatly reduced. The wealth of data generated in this project provides the DoD community the basis for developing, optimizing, and transitioning these non-chemical, laser-based surface treatments. Specifically, the extensive microstructural characterization of surfaces/sub-surface, coating adhesion testing results, and corrosion resistance testing results which were obtained for a surface structure periodicity of 1.7 micron provides a strong scientific basis for understanding the specific chemical, morphological, and microstructural changes induced by the laser-interference that affect the surface adhesion and enhance corrosion protection. Additional work is needed to fully utilize the knowledge generated in this project by exploring other structure periodicities that that explored in this pioneering study using novel, much more powerful, and high-productivity $+50 \mathrm{kHz}$ lasers.

Keywords: laser; interference; precipitates; dissolution; microstructure; aluminum; primers; coating adhesion; surface treatment; corrosion 


\section{INTRODUCTION}

Aluminum alloys need protective coatings to prevent corrosion-induced material failures (Kendig and Buchheit 2003; Nardeli et al. 2016; Nguyen and Pébère 2016; Nardeli et al. 2020). To achieve robust and functional coating system on $\mathrm{Al}$ alloys, it is essential to employ proper surface pretreatments prior to the primer application. Aluminum surfaces contain native oxides and lubricant oils, which are residual from their forming/molding operations but are detrimental to coating and adhesive joining. Surface treatments aim to modify the Al surface to attain contaminant removal, wettability with either primer or adhesive, and highly roughened or textured surfaces.

\subsection{BACKGROUND}

Aluminum-skinned aircraft are typically chemical conversion coated (MIL-PRF-5541) or anodized prior to application of coating systems or adhesive bonding. Chromate conversion coating (CCC) process is widely used as a pretreatment of Al surfaces mainly due to its strong corrosion inhibition, which is attributed to hexavalent chromium (Kendig and Buchheit 2003; Zhao et al. 2001; Laget et al. 2003; Ilevbare and Scully 2001b; Iannuzzi and Frankel 2007; Ilevbare et al. 2000; Ilevbare and Scully 2001a; Scully and Jakab 2019; Yu et al. 2000), which are highly toxic. Even when trivalent chromium is used (Type II) the clean-up and disposal of process waste is an added expense. Anodizing, in which a relatively thick alumina layer with unique columnar pores is formed (Renshaw 1961), is also used as an $\mathrm{Al}$ surface pretreatment. One common anodizing process used for $\mathrm{Al}$ is sulfuric acid anodizing (SAA). Anodization processes are much more environmentally friendly, but because they are electrochemical processes, they are still expensive to maintain and operate. Although costly, the anodized layer is highly insulating and acts as an excellent corrosion barrier. Moreover, the sealing of the anodized layer with corrosion inhibitors was found to further improve the corrosion resistance of treated Al alloys (Kalantary, Gabe, and Ross 1992; Mansfeld et al. 1998; González et al. 2000; Boisier et al. 2008; Arenas, Conde, and de Damborenea 2010; Thompson et al. 1999).

The environmental risks and costs of managing millions of gallons of spent chemical processing solutions, which often contain hexavalent chromium, other hazardous air pollutants, and/or cyanide as hazardous waste, need to be reduced. The process to prepare the substrate surface requires multiple steps including cleaning, degreasing, deoxidizing, and ending with either anodizing or chemical conversion coating. These immersion or spray processes result in long cycle times, exposure of workers to hazardous and toxic fumes, and require significant amounts of energy for spraying, agitating, and/or heating. 
Immersion tanks range from 1,000 to 40,000 gallons depending on part size and volume of work. Many of these baths contain hexavalent chromium, other hazardous air pollutants, and/or cyanide. Millions of gallons of spent chemical solutions are disposed of annually as hazardous waste. This results in a significant cost to the Department of Defense (DoD) and consumes valuable landfill space. Thus, the use of toxic chemicals during CCC and SAA processes needs to be reduced. Accordingly, chemical-free or chemical-lean pretreatment processes are now sought to replace chromate conversion coating and anodizing that require chromates and strong acids.

Laser pre-treatment of surfaces is now a widely accepted technique. Laser treatments have been successfully used on glass, ceramic (i.e., oxide), polymer, and metal surfaces (Costil et al. 2014). These treatments have been used for a variety of applications, including surface texturing (dimpling), surface roughening (via ablation), increasing bio-compatibility, alteration of wettability, tribology, modification of microstructure, and creating surfaces with reduced reflection (Berger et al. 2011); (McDaniel et al. 2015). Most of these applications have been pursued with the goal of replacing more expensive traditional surface treatments, like chemical conversion coatings and anodization.

The use of traditional, one-beam, lasers was shown to (a) be effective for the removal of contaminants (grease, oils, native oxides, etc.), thus eliminating the use of solvents (Walters 1997) and (b) enhance the adhesive bonding (Critchlow et al. 1995). However, the use of traditional lasers in surface preparation was found to yield melt cavities with micro-cracks on aluminum alloy surfaces. Advances in laser technology over the past decade have overcome some of the melt cavity and micro-crack issues by using shorter pulse durations (Jeong, Choe, and Brantley 2011).

Recently, a high-productivity laser-interference technique was used at Oak Ridge National Laboratory (ORNL) for structuring Al 5182 alloy and Carbon Fiber Polymer Composite (CFPC) surfaces (Sabau, Warren, et al. 2016) with much finer feature sizes (Daniel, Mücklich, and Liu 2003)( $1 \mu \mathrm{m}$ dia, $<3 \mu \mathrm{m}$ depth). This technique was based on harnessing the inherent constructive and destructive interference from two-or-more laser coherent beams to create an interference pattern that results in making 200 to 20,000 ridges, (pits, wells) at once, i.e., per laser spot.

\subsection{OBJECTIVE}

The main objective of this project is to demonstrate the use of a one-step process at ambient conditions based on laser-interference structuring techniques to replace the chemical conversion coating or anodization prior to the application of coating systems or adhesive bonding for Al and Ti aircraft 
components. This work addressed the SERDP WPSON-17-03 of developing a surface morphology modification technique that will enhance coating adhesion and mechanical bonding of metal surfaces by employing a newly developed laser-interference technique. This surface morphology changes brought by laser-interference are produced in one-step, without involving any additional chemical treatment, as requested by the WPSON-17-03. Laser-interference structuring (LIS) techniques was used in this project as a surface preparation method to investigate: (a) enhancement of coating/paint adhesion and (b) improvement of corrosion resistance of the coated or bonded substrate. The science goal of this study was to develop an understanding of the physical mechanisms that would improve adhesion and corrosion protection by altering the surface microstructure and topology through laser-interference structuring of A12024-T3.

To the authors' best knowledge, this study is the first to investigate the corrosion performance of primercoated LIS surfaces. The technical questions answered by this research effort were: (1) What is the optimum laser-interference induced surface morphology for enhancing coating adhesion? (2) How effective is this laser technique at cleaning the sample surface, especially in those regions where the destructive interference yields no power at all? (3) What is the surface chemistry following laser treatment? (4) What is the extent of removal of the initial native oxide? (5) What type of oxide grows subsequent to laser-interference treatment, (5) What are the sub-surface microstructural changes?, and (6) what is the corrosion resistance of coated specimens that were prepared using laser-interference techniques? This understanding is necessary to effectively transition non-chemical methods. 


\section{MATERIALS, METHODS, AND TECHNICAL APPROACH}

\subsection{MATERIALS}

For microstructural analysis, eight samples of rolled AA 2024-T3 sheet material (nominal composition of $\mathrm{Cu} 1.9$ at\%, Mg 1.52 at\%, Fe 0.12 at\%, Mn 0.27 at $\%$, Zn 0.04 at\%, bal. Al) were shear cut into 5 x $5 \mathrm{~cm}$ sized samples. The 2024-T3 0.032" gauge sheet material was certified to meet the ASTMB209, AMSQQA250/4, and AMS4037 standards by the supplier. No cleaning was performed before or after cutting the samples. Samples were examined in the as-received condition after cutting and again after laser processing.

For coating adhesion and corrosion testing, bare Al 2024-T3 panels with the surface area of $50 \mathrm{~mm} \times 50$ $\mathrm{mm}$ or $75 \mathrm{~mm} \times 75 \mathrm{~mm}$ and the thickness of $0.81 \mathrm{~mm}(0.032 \mathrm{in})$ were obtained from an external vendor. All of the Al 2024-T3 with control pretreatments, namely CCC and SAA, were obtained from the Q-LAB vendor (Westlake, $\mathrm{OH}$ ). According to the vendor's report, $\mathrm{CCC}$ treatment was completed using a hexavalent chromium agent according to MIL-DTL-5541 type I (with hexavalent Cr), and SAA panels were sealed using hot water without corrosion inhibitors according to MIL-DTL-8625 type II, Class 1. For the laser structuring, the 2024-T3 $0.813 \mathrm{~mm}$ (0.032 in) gauge sheet material was certified to meet the ASTM B209, AMS-QQA250/4, and AMS4037 standards by the supplier. The thicknesses of CCC and SAA Al $100 \mathrm{~mm} \times 150 \mathrm{~mm}$ panels were also $0.813 \mathrm{~mm}$ (0.032 in). The CCC and SAA panels were cut to the size of $50 \mathrm{~mm} \times 75 \mathrm{~mm}$ using a shear cutter. The thickness of chromated and anodized layers was measured at the center and four spots diagonally $1 \mathrm{~cm}$ away from each corner (total five spots) per panel. A commercial thickness gage (DeFelsko) using ultrasonic reflection for the thickness measurement was used. In 15 CCC panels, the average thickness of chromate layer was below the detection limit, i.e., 0.5 $\mu \mathrm{m}$. In 15 SAA panels, the average thickness of anodized layer was approximately $4 \mu \mathrm{m}$. A commercially available CA7233 primer kit, compliant to MIL-PRF-23377 Type I Class C2 specification, was selected in this work. Desoprime ${ }^{\text {TM }}$ HS CA7233 Military Epoxy Primer was prepared by mixing by volume one part of CA 7233A base and one part of CA 7233B activator. Primers for this military specification were manufactured by PPG Industries, Inc. This primer product contained strontium chromate as corrosion inhibitor. 


\subsection{INTRODUCTION OF LASER-INTERFERENCE STRUCTURING}

In this study, a new laser technique is proposed as a surface preparation technique not only to attain cleaning but also surface structuring at a much finer spatial resolution than those attained by the traditional one-beam laser setup. The one-beam laser techniques produce geometrical features of sizes that are given by the laser spot size. For example, the line scan width is given by the laser spot diameter for scanning. Also, the size of dimples for single laser shots is also given by the laser spot diameter. The proposed laser-interference technique offers the possibility of structuring the specimen surface with much finer spatial resolution. The main size features are summarized in Table 1 for both the one-beam laser technique and for laser interference techniques. The beam size dependence of the spatial resolution of the one-beam techniques severely limits their application to small beam sizes, usually between 0.08 and 1 $\mathrm{mm}$, in order to attain a structuring with a sub $\mathrm{mm}$ resolution. The laser interference power profile was created by splitting the beam and guiding those beams to the sample surface by overlapping each other with defined angles to each other. The schematic from Figure 1 indicates the system components and illustrates the optical setup used in this project to create laser interference on the specimen surface. As needed, the laser pulse fluence (pulse energy per unit area) was increased by using two identical focal lenses in each path of the beams to focus them to a smaller spot from its original size of $8 \mathrm{~mm}$ (Figure 1). The coherent beams create an interference pattern instead of just adding their intensity. This allows a microscopic modulation and creates a light pattern without loss of energy during the interference process. The periodicity between power peaks and laser-interference induced undulations is defined by the

wavelength, $\lambda$, and the angle, $\alpha=\beta+\beta_{1}$, between the two laser beams, as: ${ }^{d=\lambda} /(2 \sin (\alpha / 2))$. Assuming wavelengths of 355 to $1,064 \mathrm{~nm}$ and beam angles of 6 to $72^{\circ}$, other features of the structured surface morphology than those included in Table 1, include: (a) undulation spacing of $0.4-20 \mathrm{um}$, (b) structured area of $0.27 \mathrm{~cm}^{2} / \mathrm{shot}$ (area of a $6 \mathrm{~mm}$ diameter spot) and scanning speed of up to $162 \mathrm{~cm}^{2} / \mathrm{min}$ [12].

Table 1. Features and differences of proposed technique from conventional laser ablation techniques

\begin{tabular}{|l|l|l|}
\hline Feature & One-beam laser & Advantages of laser-interference \\
\hline Patterns and shape & $\begin{array}{l}\text { Shape of patterns identical to the } \\
\text { laser spot shape }\end{array}$ & $\begin{array}{l}\text { New patterns can be dot- or line- } \\
\text { shaped }\end{array}$ \\
\hline $\begin{array}{l}\text { Geometrical feature } \\
\text { size }\end{array}$ & $\begin{array}{l}0.5 \text { to } 1 \mathrm{~mm} \text { identical to the laser } \\
\text { spot size }\end{array}$ & $\begin{array}{l}\text { Feature size: 1 to } 500 \mathrm{~nm} \\
\text { Feature density: 20 to 2,000 per } \mu \mathrm{m}\end{array}$ \\
\hline
\end{tabular}




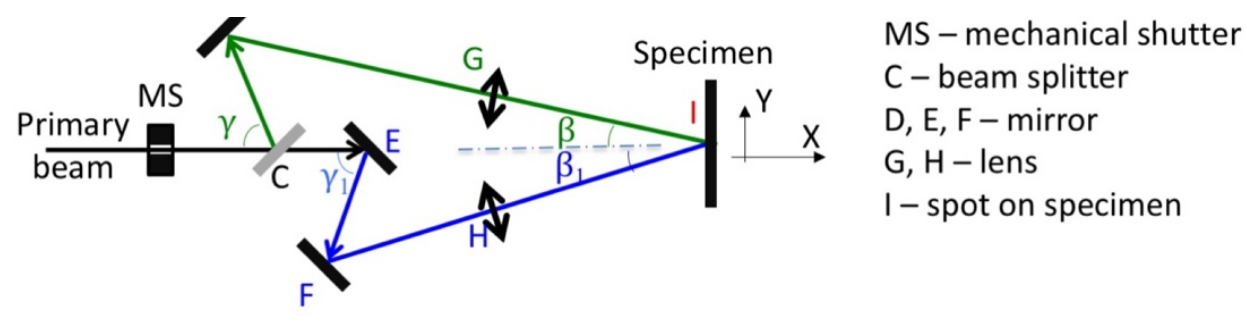

Figure 1. Schematic of the setup of laser interference system.

\subsection{PHYSICAL PHENOMENA FOR LASER-INTERFERENCE STRUCTURING OF METALS}

Briefly, the laser-interference structuring of the Al alloys can be explained by the induced photothermal effect that creates a periodic heat treatment according to the spatial power distribution due to the waveinterference (Sabau, Greer, et al. 2016). As a result of photon absorption and metal properties, Al will melt and evaporate when its temperature rises above its melting point and saturation temperature at atmospheric pressure, respectively. (Lasagni et al. 2007) conducted a combined experimental and computational study of the laser-interference for steel, $\mathrm{Cu}$, and $\mathrm{Al}$ and investigated the role of fluid dynamics and thermal conduction in the formation of interference-based structuring of the surface. Numerical simulations were conducted for the heat transfer by considering simplifying assumptions on the heat required for vaporization and melting, without a detailed treatment of the free-surface flows induced by melting. It was found that three parameters (Lasagni et al. 2007), namely: (1) the thermal diffusion length, (2) thermal gradient between locations at maximum and minimum power distribution, and (3) residence time of the Marangoni flow of molten metal, govern the type of surface morphology that would be induced by the power interference distribution on the metal surface. The results presented by (Lasagni et al. 2007) indicate that the structuring is produced by a surface tension driven mechanism induced by the thermal gradient between maxima- and minima-interference induced and are summarized in the remainder of this section. The thermal diffusion length, $L_{t h}$, as computed using the time-scale given by the pulse duration $\left(\tau_{p}\right)$ and thermal conductivity $(k)$, as $L_{t h}=\sqrt{k \tau_{p}}$, was found to limit both the height/depth of interference-induced structures (for 1 pulse) and the minimal achievable periodic length, without loss uniformity, of interference-induced structures. For $\mathrm{Al}$ exposed to a $10 \mathrm{~ns}$ pulse of energy $L_{t h}$ was estimated to be 0.96 microns. The minimal achievable periodic length was found to be higher than $2 L_{t h}$. Another important factor governing the structuring mode for metals, is the effective convection time, defined as the time available for the fluid dynamics due to Marangoni forces to drive the molten metal over half of the interference periodicity. For 
a laser fluence of $1.6 \mathrm{~J} / \mathrm{cm}^{2}$, the effective convection time was estimated to be approximately $25 \mathrm{~ns}$ (Lasagni et al. 2007). Based on the experimental and model simulation results (Lasagni et al. 2007), the surface morphology due to laser-interference processing is dependent on the laser fluence as indicated in Figure 2 (Sabau, Greer, et al. 2016), as follows: (a) at low laser fluences, the material melts and stays at maxima locations as the temperature gradients are small and Marangoni forces are small, (b) at moderate laser fluences, the material starts to be removed from interference maxima locations resulting in a double-peak geometry, and (c) at high fluences, the residence time of the metal is increased and the two fluid fronts coming from the two maxima-interference coalesce (and it could also be affected by the evaporation and vapor pressure).

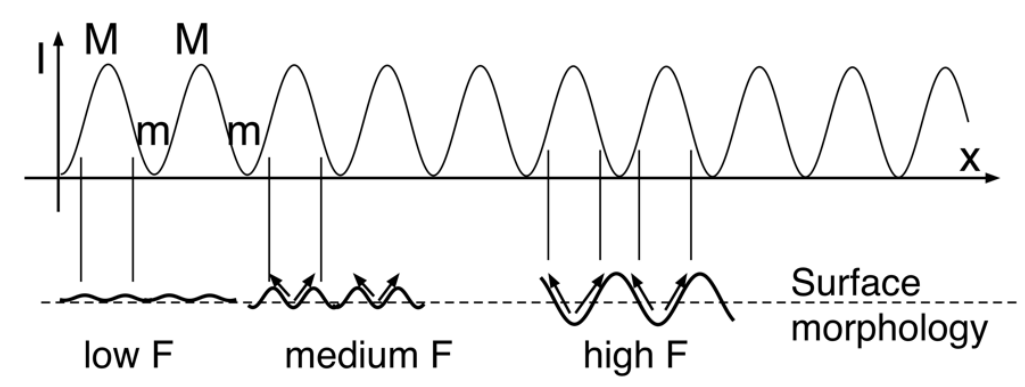

Figure 2. Schematic of laser-interference induced structuring patterns as a function of laser fluence (F).

The major gap in the current-state-of-the art lays in the application of the laser-interference technique to the as-received $\mathrm{Al}$ and $\mathrm{Ti}$ surfaces. In addition to the basic physical phenomena, which were reviewed in the above paragraph for the laser-interference structuring of Al with well-polished surfaces, it has to be mentioned that additional phenomena take place during the laser-interference structuring of the $\mathrm{Al}$ in the as-received condition. For the as-rolled A15182 sheet material, the resulting surface morphologies, which were noted at ORNL (Sabau, Greer, et al. 2016)due to the melting and subsequent fluid dynamics at the microscale, were:

(a) At medium laser fluences and or medium number of pulses, regularly spaced pits (or pin-holes) embedded within a woven type of network were observed. These regularly spaced pin-holes combined with woven network structures were found to occur at a much larger scale than the periodic spacing expected due to the energy interference.

(b) At high fluences and/or large number of pulses per spot, significant melting was evidenced by wavy or drop-like formations, which were very different from the surface topology of the asreceived condition. 
Additional phenomena include the energy absorption and the evaporation of surface contaminants, such as lubrication oils from prior sheet fabrication processes. This film evaporation, which requires latent heat input, would have the effect of decreasing the local temperatures in the metal surface and inducing a non-uniform temperature distribution, at least for the first pulses. The (non)uniformity of the lubrication films would affect the overall energy available locally for the surface treatment. In addition, there is a possibility of chemical reactions between the surface contaminants and substrate. Finally, the subsequent oxidation of the laser-interference treated Al may be non-uniform, depending on the local temperature evolution during laser surface treatment.

\subsection{QUALITY OF LASER-INTERFERENCE STRUCTURING OF METALS}

Concerning the quality and metrics of the laser-interference structures, the following considerations can be made. (Lasagni et al. 2007) and (Bieda, Beyer, and Lasagni 2010) investigated the physical phenomena that lead to laser-interference structuring for stainless steel and pure metals $(\mathrm{Al}, \mathrm{Cu}$, and $\mathrm{Ti})$ by employing an energy transport model that included simplified assumptions for heat losses due to vaporization and melting for pure metals. (Lasagni et al. 2007) employed lubrication flow correlations, without a detailed treatment of the free-surface flows induced by melting, to illustrate the effect of Marangoni forces during laser-interference structuring. Lasagni et al. (2007), (Bieda, Beyer, and Lasagni 2010), and D'Alessandria et al. (2008) It was indicated that the following factors may govern the type of surface morphology that would be induced by the laser-interference for a single laser pulse (Lasagni et al. 2007; D’alessandria, Lasagni, and Mücklich 2008):

(1) temperatures at interference maxima and minima, $\mathrm{T}_{\mathrm{Max}}$ and $\mathrm{T}_{\mathrm{Min}}$, respectively,

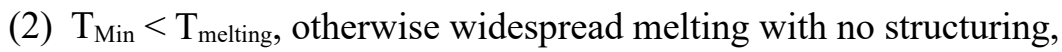

(3) $\mathrm{T}_{\mathrm{Max}}<\mathrm{T}_{\text {vap }}$, otherwise evaporation may reduce the structures depth $\left(\mathrm{T}_{\mathrm{vap}}\right.$ is the evaporation temperature). Bieda et al. (2010) indicated that a for a periodicity of $5 \mu \mathrm{m}$ there would be a decrease in the structuring quality at fluences $>1.3 \mathrm{~J} / \mathrm{cm}^{2}$ due to the evaporation effects expected at the maxima interference.

(4) if $T_{M a x}>T_{\text {vap}}$, the recoil pressure may depress further down the surface of molten metal, increasing the structure depth,

(5) the thermal diffusion length, $L_{t h}$, which can be estimated using the time-scale given by the pulse duration $\left(\tau_{p}\right)$ and thermal diffusivity $(\alpha)$, as $L_{t h}=\sqrt{\alpha \tau_{p}}$, was considered as a measure to quantify the "heat localization"; minimum periodicity of the structuring was estimated to be $d_{\text {min }}=2 L_{t h}$. For a $10 \mathrm{~ns}$ pulse of energy, $L_{t h}$ was estimated to be $L_{t h}=0.96 \mu \mathrm{m}$, yielding $d_{\text {min }} \sim 2 \mu \mathrm{m}$ (Lasagni et al. 2007) 
(6) appropriate thermal gradient over half the interference periodicity, $\left(\mathrm{T}_{\mathrm{Max}}-\mathrm{T}_{\mathrm{Min}}\right) /(0.5 \mathrm{~d})$ to establish appropriate Marangoni flow, and

(7) appropriate residence time of the Marangoni flow of molten metal.

An analysis is presented in the remainder of this section to infer structuring characteristics for laserinterference processing of A12024, such as fluence thresholds and minimum periodicity, based on the data and correlations developed for one laser pulse pure Al (Lasagni et al. 2007) and (D'alessandria, Lasagni, and Mücklich 2008). It is worth noting that although a similar laser to that used in our study was used in (Lasagni et al. 2007) and (D'alessandria, Lasagni, and Mücklich 2008), multiple laser shots were used in our study. For one laser pulse, the structure depth, $h$, was found to vary with fluence and periodicity. For polished pure Al specimens and a periodicity of $4.7 \mu \mathrm{m}$, the structure depths would increase from $\sim 50 \mathrm{~nm}$ to $700 \mathrm{~nm}$ and 1,270 $\mathrm{nm}$ at laser fluences of 1.5, 1.68, and $1.8 \mathrm{~J} / \mathrm{cm}^{2}$, respectively (Lasagni et al., 2007). For degreased, rough, as-received cold-rolled, specimens and a periodicity of $2.2 \mu \mathrm{m}$, the structure depths would increase from $\sim 259 \mathrm{~nm}$ to $480 \mathrm{~nm}$ and $700 \mathrm{~nm}$ at laser fluences of $0.9,1.12$, and $1.57 \mathrm{~J} / \mathrm{cm}^{2}$, respectively (D'Alessandria et al., 2008). D'Alessandria et al., 2008 found that the structuring was attained at lower fluences for rougher surfaces than for polished surfaces. This was explained by the intrinsic smaller reflectivity for rougher surfaces than that for the polished surfaces. Analyzing this type of data from (Lasagni et al. 2007) and (D'alessandria, Lasagni, and Mücklich 2008), the variation of structure depth with laser fluence can analytically be described with a hyperbolic tangent function, i.e., exhibiting a sharp increase over a small fluence range of $\sim 0.3 \mathrm{~J} / \mathrm{cm}^{2}$, as:

$h(F, d, S C)=0.5\left(h_{\max }+h_{\min }\right)+0.5\left(h_{\max }-h_{\min }\right) \tanh \left[16\left(F-F_{t h}(d, S C)\right)\right]$,

where the $F_{t h}$ indicate a threshold fluence, at which $h \sim 0.5\left(h_{\min }+h_{\max }\right)$, and SC indicate a surface condition (e.g., polished, rough, etc.), respectively. An effective structuring, with $h \sim 0.5 h_{\max }$ to $h_{\max }$, would be attained for fluences larger than $F_{t h}$. As indicated in Table 2, the threshold fluence, $F_{t h}$, was found to vary with the structure depth and surface condition. Based on minimum fluence reported, inferred threshold fluence, depth of structures $\left(h_{\min }, h_{\max }\right)$, the following observation can be made that can be applicable to our study, in which rough surfaces and small periodicities were considered: (a) rougher surfaces would require smaller laser energy for interference structuring, (b) smaller periodicities would require larger laser energy for structuring, and (c) smaller $h_{\text {max }}$ are expected for smaller periodicities. 
Table 2. Survey of structure depth at various periodicities and fluences for one-pulse laserinterference processing of pure Al

\begin{tabular}{|c|c|c|c|c|c|}
\hline $\begin{array}{c}\mathrm{d}_{\exp } \\
{[\mu \mathrm{m}]}\end{array}$ & $\begin{array}{c}\mathrm{h}_{\min } \\
{[\mu \mathrm{m}]}\end{array}$ & $\begin{array}{c}F_{\min } \\
{\left[\mathrm{J} / \mathrm{cm}^{2}\right]}\end{array}$ & $\begin{array}{c}F_{t h} \\
{\left[\mathrm{~J} / \mathrm{cm}^{2}\right]}\end{array}$ & $\begin{array}{c}\text { Surface } \\
\text { condition }\end{array}$ & Reference \\
\hline 2.2 & 0.29 & 0.9 & 1.2 & rough & \multirow{3}{*}{$\begin{array}{l}\text { (D’alessandria, } \\
\text { Lasagni, and } \\
\text { Mücklich 2008) }\end{array}$} \\
\hline 3.4 & 0.43 & 0.97 & 1.37 & rough & \\
\hline 3.4 & 0.28 & 1.42 & 1.75 & polished & \\
\hline 4.7 & 0.05 & 1.5 & 1.68 & polished & $\begin{array}{c}\text { (Lasagni et al. } \\
\text { 2007) }\end{array}$ \\
\hline
\end{tabular}

The thermophysical properties for the A12024 alloy were obtained based on thermodynamic simulation using the Al material database Computherm and microstructure model in ProCAST, as shown in (Sabau, Meyer, and Leonard 2020). The thermophysical property data indicate that A12024 undergoes phase changes over a very wide range of temperatures $\left(\mathrm{T}_{\text {Solidus }}=508{ }^{\circ} \mathrm{C}\right.$ and $\mathrm{T}_{\text {Liquidus }}=640{ }^{\circ} \mathrm{C}$ for the Scheil model). The thermal diffusivity for the A12024 was estimated to be $5.25 \mathrm{e}-5 \mathrm{~m}^{2} / \mathrm{s}$, which is in excellent agreement with experimental data. For an $8 \mathrm{~ns}$ laser pulse, $L_{t h} \sim 0.65 \mu \mathrm{m}$ and hence $d_{\min } \sim 1.3 \mu \mathrm{m}$ would be expected. The spectral optical properties of the surface A12024 could be different than those of pure Al. The reflectivity at a wavelength of $355 \mathrm{~nm}$ was measured to be $\sim 70 \%$ for the rough sample and $\sim 92 \%$ for the polished sample (D'Alessandria et al., 2008). The reflectivity of $92 \%$ for pure $\mathrm{Al}$ is in excellent agreement to that published. The normal spectral reflectivity for cold-rolled A12024 specimens was not measured. However, the normal spectral reflectivity for A12024 specimens with a surface roughness of $\sim 0.18 \mu \mathrm{m}$ was measured to be $75-80 \%$ (Marshall 1963), which is close to that of $\sim 70 \%$ measured for the cold-rolled Al (D’Alessandria et al., 2008). 


\section{PROCESSING EQUIPMENT AND EXPERIMENTAL PROCEDURES FOR SURFACE PREPARATION AND COATING APPLICATION}

\subsection{LASER EQUIPMENT AND OPTICAL SETUP FOR LASER-INTERFERENCE STRUCTURING}

A 10Hz Q-switched Nd:YAG laser (Quanta-Ray PRO 230, Spectra Physics) was used in this study. The fundamental laser emission with the wavelength of $1064 \mathrm{~nm}$ was transformed to $355 \mathrm{~nm}$ using non-linear crystals. At $355 \mathrm{~nm}$ wavelength, the laser pulse duration was $\sim 8 \mathrm{~ns}$. The laser interference power profile was created by splitting the main laser beam and guiding the two beams to the sample surface by overlapping each other with defined angles (Meyer, Sabau, and Daniel 2019). The coherent laser beams create an interference pattern in the overlap spot. As shown in Figure 3, The laser pulse fluence (pulse energy per unit area) was increased by using identical lenses in each beam path in order to decrease the spot size to $5 \mathrm{~mm}$ or $6 \mathrm{~mm}$ from the original beam size of $8 \mathrm{~mm}$ (Sabau, Greer, et al. 2016; Meyer, Sabau, and Daniel 2019). The periodicity between power peaks and the resulting laser-interference induced undulations $(d)$, is defined by the wavelength, $\lambda$, and the angle, $\alpha$, between the two beams, as $d=$ $\lambda / 2 \sin (\alpha / 2)$.

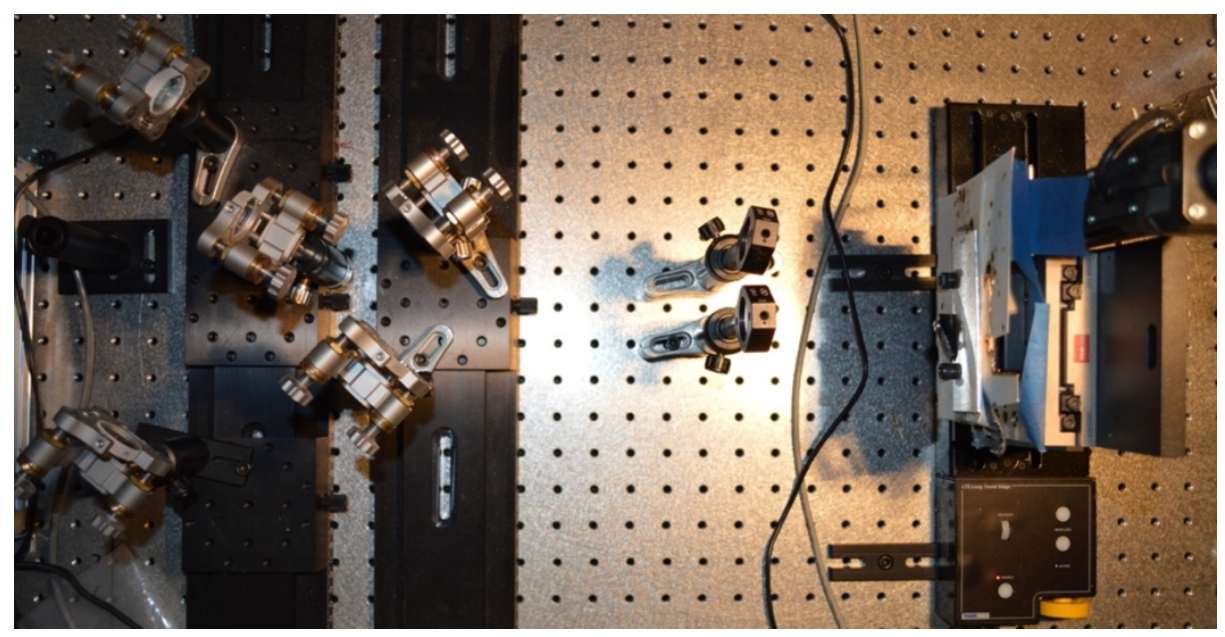

Figure 3. Picture of the setup of laser interference system for a beam angle of $\sim 8.5^{\circ}$. 


\subsection{LASER-INTERFERENCE STRUCTURING PROCEDURE}

As-received Al panels, which contain lubrication oil residues from prior rolling operations and ensuing surface contaminants, were laser processed without any prior surface cleaning. The specimens were processed in raster mode by translating the sample while the laser fired pulses at $10 \mathrm{~Hz}$ frequency. At the end of each line scan, the specimen was then positioned straight up from last spot such that the next line scan will be laser processed. The overlap between adjacent rows of scans was $1 \mathrm{~mm}$. The scan direction was identical to that of the rolling direction. The beam angle was $12^{\circ}$, for a theoretical periodicity of the structures of $\sim 1.7 \mu \mathrm{m}$. In this work, two laser fluences of $F_{1}=1.238$ and $1.782 \mathrm{~J} / \mathrm{cm}^{2}$ per pulse were used by varying the laser spot size ( $d_{b}=6$ and $5 \mathrm{~mm}$, respectively) while keeping the same average power of $3.5 \mathrm{~W}$. The pulse repetition rate was $f_{L}=10 \mathrm{~Hz}$. To quantify the effect rastering speed, $U$, of the laser beam on the energy deposited on the specimen surface, the two process variables were introduced: (a) the number of pulses that a local area is exposed, $N_{P}(U)=d_{b} \cdot f_{L}{ }_{U}$, and (b) the accumulated fluence on the specimen surface, $F_{A}=N_{P} \cdot F_{1}$, where the fluence of each shot is $F_{1} . N_{P}$ and $F_{A}$ are given in Table 3 for all laser interference structuring (LIS) conditions considered in this study. The specimen labels for each LIS condition, which were used for the corrosion study, are also shown in Table 3. As the raster speed is increased, the surface is exposed to a smaller number of shots and smaller accumulated fluences.

Table 3. Energy metrics for several raster speeds for laser fluences $F_{1}$ of 1.782 and $1.238 \mathrm{~J} / \mathrm{cm}^{2}$ and identification of specimen process conditions (process ID) for corrosion testing

\begin{tabular}{|c|c|c|c|c|c|c|}
\hline$F_{1}\left[\mathrm{~J} / \mathrm{cm}^{2}\right]$ & \multicolumn{3}{|c|}{1.782} & \multicolumn{3}{c|}{1.238} \\
\hline$U[\mathrm{~mm} / \mathrm{s}]$ & Sample ID & $N_{P}$ & $F_{A}\left[\mathrm{~J} / \mathrm{cm}^{2}\right]$ & $*$ Sample ID & $N_{P}$ & $F_{A}\left[\mathrm{~J} / \mathrm{cm}^{2}\right]$ \\
\hline 2 & - & - & - & LIS 2B & 30 & 37.14 \\
\hline 4 & LIS 4A & 13 & 22.28 & LIS 4B & 15 & 18.57 \\
\hline 6 & LIS 6A & 8 & 14.85 & LIS 6B & 10 & 12.38 \\
\hline 8 & - & - & - & LIS 8B & 7 & 9.28 \\
\hline 10 & LIS 10A & 5 & 8.91 & - & - & - \\
\hline
\end{tabular}

* Samples LIS 2B, 4B, 6B and 8B were acetone wiped and dry-wiped with lint-free wipes immediately after the LIS.

\subsection{PRIMER COATING PROCEDURE}

Both the laser-structured specimens and the controlled specimens were stored in plastic cases in order to avoid air contaminants and humidity effects for several days, weeks, and even months prior to coating 
application. Each specimen was laid in its own plastic case with the LIS surface facing up such that the LIS surface did not touch the plastic case surface. The storage duration of the LIS specimens prior to coating application is often referred to in industry as open-time, $t_{o}$. The open-time was recorded for all LIS specimens in order to study any possible degradation of the coating adhesion with the open-time. The procedure for the primer coating operation on Al panels was conducted as follows. For pre-cleaning of control specimens, the Al surface was rinsed with ethanol and gently wiped with a dust-free cloth. The surface of the laser structured specimens was not cleaned at all prior to the laser processing. The cleaned control panels and "uncleaned" laser structured panels were loaded on a paint rack and spray-coated with CA7233 primer using a spray gun operated with the nozzle pressure of $0.138 \mathrm{MPa}$. The painting of primer was conducted within the allowable temperature range $\left(20-30^{\circ} \mathrm{C}\right)$ and below the upper humidity limit of $65 \%$. The coated $\mathrm{Al}$ panels were then dried in a fume hood for 24 hours to allow evaporation of volatile species and subsequently cured in an oven at $60^{\circ} \mathrm{C}$ for 24 hours.

All LIS panels were stored in plastic cases in order to minimize airborne contaminants and humidity effect prior to coating application for up to 70 days. The plastic cases were not airtight, and no desiccant was used. Each specimen was placed in its own plastic case with the LIS surface facing up such that the LIS surface would not touch the plastic case surface, in order to reduce further surface contamination during storage. The storage duration of the LIS specimens prior to coating application was found to have a negligible effect on coating adhesion (Sabau, Jun, and McClurg 2020), hence its effect was not further investigated in this study.

The procedure for the application of the primer coating on all pretreated Al panels was conducted as follows. The CCC and SAA Al panels were rinsed with ethanol and gently wiped with dust-free cloth prior to the primer application. Two batches of LIS specimens were prepared: (A) without any additional wiping/cleaning, and (B) with additional acetone cleaning right after the laser structuring followed by dry-wipe with lint-free wipes. LIS panels from both batches were not cleaned by any means prior to the primer application. The panels were then loaded on a mesh rack to allow adequate air flow during the coating and spray-coated with primer using a paint gun operated with the nozzle pressure of $0.138 \mathrm{MPa}$ (20 psi) at the allowable temperature range of $20-30^{\circ} \mathrm{C}$ and below the humidity limit of $65 \%$. The coated Al panels were then dried in a fume hood for 24 hours to allow evaporation of volatile species and subsequently cured in an oven at $60^{\circ} \mathrm{C}$ for 24 hours. After this oven curing, the coating thickness of primer on Al panels was measured using the same thickness gage and procedure described in the materials section, and the average coating thickness was obtained from five measurements per panel. It 
should be noted that the coating thickness on the SAA panels includes both primer and $\sim 4 \mu \mathrm{m}$ thick anodized layer. The target thickness of primer coating was below $23 \mu \mathrm{m}$. 


\section{MATERIALS CHARACTERIZATION EQUIPMENT AND PROCEDURES}

\subsection{ELECTRON MICROSCOPY \& MICROANALYSIS}

A field emission scanning electron microscope (Hitachi S4800 FE-SEM) was used to obtain data on morphology and elemental composition changes of laser processed surfaces of Al 2024-T3 specimens. To minimize the interaction volume for secondary electron imaging the accelerating voltage for scanning electron microscopy (SEM) imaging of the surface was chosen to be $5 \mathrm{kV}$. The interaction volume depth

in $\mathrm{Al}$ at $30 \mathrm{kV}$ has a $\mathrm{z}$-depth of $\sim 1.5 \mu \mathrm{m}$ versus $\sim 300 \mathrm{~nm}$ at $5 \mathrm{kV}$. The Hitachi S4800 was also equipped with an energy dispersive spectrometer (EDS) for X-ray mapping, which was used in this study to provide data on near-surface elemental composition. The accelerating voltage was changed to $30 \mathrm{kV}$ for additional SE imaging and EDS analysis.

Focused ion beam (FIB) milling was used to prepare site specific electron transparent scanning transmission electron microscopy (STEM) specimens similar to specimen preparation reported previously (reference Leonard and Hellmann). A Hitachi NB5000 FIB/SEM with ion beam column operated at $40 \mathrm{kV}$ and various probe currents for material removal and final thinning. STEM and EDS were performed with a Thermo Talos F200X operated at $200 \mathrm{keV}$. STEM micrographs from the high angle annular detector produce atomic number contrast and are termed 'Z-contrast' images. Bright field (BF) micrographs were also acquired during the STEM and EDS analysis.

\subsection{X-RAY PHOTOELECTRON SPECTROSCOPY}

Chemical changes to the AA 2024-T3 surface induced by laser treatment were examined in detail in this study using X-ray photoelectron spectroscopy (XPS) performed with a Thermo Scientific Model K-Alpha XPS instrument. This instrument uses a monochromated, micro-focusing, $\mathrm{A} 1 \mathrm{~K}_{\alpha} \mathrm{X}$-ray source (1486.6 $\mathrm{eV})$ with a variable $\mathrm{X}$-ray spot size $(30-400 \mu \mathrm{m})$. Analyses of all samples used the largest X-ray spot size $(400 \mu \mathrm{m})$ for maximum signal and to obtain an average surface composition over the largest possible area. Photoelectrons were energy analyzed and detected with a hemispherical analyzer equipped with a 128-channel detector system. The base pressure in the analysis chamber is typically $3 \times 10^{-10} \mathrm{mbar}$. Al alloy samples were mounted to the sample platen using metal clips. Wide energy range survey spectra $(0-$ $1350 \mathrm{eV}$ ) were acquired for qualitative and quantitative analysis using a pass energy setting on the analyzer of $200 \mathrm{eV}$. For assessing chemical bonding of identified elements, narrow energy range core 
level spectra were acquired with a pass energy setting of $50 \mathrm{eV}$. For depth profiling, an Ar-ion gun operated at $2 \mathrm{kV}$ was employed. In separate experiments, the $2 \mathrm{kV} /$ high current setting of the gun was calibrated at $10 \mathrm{~nm} / \mathrm{min}$ using $100 \mathrm{~nm}$ thick standard $\mathrm{SiO}_{2}$ films. Depth profiling experiments were run by alternately sputtering the sample for a given time, blanking the ion gun, and then collecting spectra of the elements of interest. Depth scales on the profiles were determined using the calibrated etch rate of 10 $\mathrm{nm} / \mathrm{min}$ and the etch times measured during the experiment. The depth scales used in the figures are only accurate if the predominantly $\mathrm{Al}$ - and $\mathrm{Mg}$-oxide-rich surfaces sputter at the same rate as the $\mathrm{SiO}_{2}$ standard films. The actual depth is likely greater than or less than the depth scale shown but is good for sample-tosample comparisons in this report. Spectra for depth profiles are collected using "snapshot" mode where the entire width of the 128-channel detector acquires the full core level spectrum. In snapshot mode data acquisition time is greatly reduced at the expense of some energy resolution. Data were collected and processed using the Thermo Scientific Avantage XPS software package (v 4.61). When necessary, spectra were charge corrected by setting the main C 1s core level peak to $284.8 \mathrm{eV}$. Peak fitting of the Al $2 p$ depth profile data was employed to separate the contributions of Al-metal (binding energy $72 \mathrm{eV}$ ) and Al-oxide (binding energy $74.5 \mathrm{eV}$ ).

Precision and accuracy of XPS surface analysis are frequently discussed. Most reviews over the last 30 years indicate that while in terms of quantitative accuracy, XPS can be off 5-15\% (depending on the core level being monitored and concentration of the element), its precision is less than $2 \%$. When comparing the same type of measurement made on the same material using the same instrument, high precision can be achieved. In this chapter, all the XPS measurements were made on the same lot of material (i.e., a single rolled sheet of Al 2024) using the same experimental conditions on the same instrument; hence the sample-to-sample differences observed are significant. This can be supported by two other observations.

First, on a previous analysis of a different but similar Al/Mg alloy (Meyer et al., 2019), several repeats on the baseline material were run and gave almost identical results for the depth profiles. Second, in an extended study of this alloy, but not reported in this study, two depth profiles, which were obtained at two different locations on the same sample, showed insignificant differences, aside from the data that was obtained at the top 1-5 nm of the samples since slightly different amounts of carbon were on the outer surface (due to the different packaging of the second specimen).

\subsection{PROFILOMETRY AND ROUGHNESS ANALYSIS PROCEDURES}

A WYKO NT9100 surface profilometer was used to obtain surface roughness data for the alloy surfaces in the as-received condition, i.e., without any laser processing, and after laser-interference processing. 
Wyko optical surface profilometer systems, which were supplied by Veeco Instruments Inc., are noncontact optical profilers that can be used to measure a wide range of surface topographical features. This surface profiler is a non-contact optical profiler based on light interferometry that can be used to measure a wide range of surface topographical features. The vertical resolution was $<0.1 \mathrm{~nm}$ with the Root-meansquare (RMS) repeatability of $0.05 \mathrm{~nm}$, as indicated in the instrument brochure based on phase-shifting interferometry difference measurement on a SiC reference mirror. For a field of view of 174 x $130 \mu \mathrm{m}^{2}$, the resolution in the lateral directions was $\sim 130 \mathrm{~nm}$. The area chosen for surface profiling was located along the centerline of a laser scan. A magnification of 50X was used for surface profiling. The average roughness, or arithmetical mean deviation of the roughness profile, $R_{a}$, root-mean-square deviation of the roughness profile, $\mathrm{R}_{\mathrm{q}}$, maximum roughness, $\mathrm{R}_{\mathrm{z}}$, and maximum height of the roughness profile, $\mathrm{R}_{\mathrm{t}}$, were included in the analysis. $\mathrm{R}_{\mathrm{a}}$ was calculated by averaging the absolute height variation within the sampling length by excluding a few outlying points so that the extreme points have no significant impact on the final results. By contrast, profile extremes have a much greater influence on $\mathrm{R}_{\mathrm{z}}$, which is an absolute vertical variation between the maximum profile peak height and the maximum profile valley depth along the sampling length. Usually, $R_{z}$ averages the five highest peaks and the five deepest valleys. $R_{t}$ is height variation between the maximum profile peak height and the maximum profile valley depth along the sampling length.

\subsection{COATING ADHESION TEST PROCEDURES AND PROCESS VARIABLES}

Following oven curing, the thickness of the primer on Al panels was measured using the same DeFelsko thickness gage that was used for measuring the anodized thickness layer. For each panel, the primer thickness was measured at the center and 4 spots diagonally $1 \mathrm{~cm}$ away from each corner for a total of five measurements per panel. The average Coating Thickness (CT) was obtained from these five measurements. It should be noted that the coating thickness of SAA panels includes both that of the primer and $\sim 4 \mu \mathrm{m}$ thick anodized layer. Per manufacturer specifications, the recommended thickness of the primer coating is 15 to $30 \mu \mathrm{m}$.

The adhesion performance of primer coating was tested and assessed according to American Society for Testing and Materials (ASTM) ASTM D3359 standard which specifies X-cut and cross-hatch scratch test procedures and the criteria of adhesion rating. The adhesion rating of both scratch tests is based on 0-5 scale increasing by 1 with 0 and 5 assigned for the lowest and highest adhesion, respectively. The X-cut tape adhesion testing was conducted according to procedures from section seven of ASTM D3359. An Xcut was made through the coating to the substrate using a pencil type scribing tool with carbide stylus, 
then a pressure-sensitive tape was applied over the cut. Within 60 to $120 \mathrm{~s}$ of tape application, tape was removed by pulling it off rapidly back upon itself at as close to an angle of $180^{\circ}$ as possible. The coating adhesion rating was assigned by examining the $\mathrm{X}$-cut coating area, which was removed from the substrate or previous coating, in accordance with the scale classification shown from ASTM D3359 (section 7). The X-cut test is easier to perform than the cross-hatch test.

The cross-hatch adhesion tests were conducted according to ASTM D3359, Section 12. A Paint Adhesion Test (PAT) kit was purchased from Paul N. Gardner Company, Inc., including the PA-2000 handle and the cutter PA-2053 (1.0 mm blade spacing with 11 teeth). The coating adhesion rating for the cross-hatch test was evaluated by examining the grid area for removal of coating from the substrate using a magnifier, and rating the adhesion for according with the scale classification. The X-cut test is considered in industry as a less severe test than the cross-hatch test. Thus, more specimens were tested for the cross-hatch test than for the X-cut test, once the X-cut tests indicated that the laser structured specimens would pass this test. 


\section{SURFACE TOPOLOGY CHARACTERIZATION}

\subsection{Surface morphology of AL 5182 obtained by spot-by-spot laser-interference structuring}

In the SEM micrograph of Figure 4 the typical topography features of the as-received unprocessed aluminum surface are shown, such as rolling marks in the vertical direction and a high density of microcracks. It should be mentioned again that the as-received Al surface was laser processed without any additional surface preparation. In order to illustrate the laser structuring capability with the current optics setup with increasing number of pulses per spot, optical micrographs for three AA 5128 specimens are shown Figure 5(a, b, and c), for number of pulses per spot of 1, 2, and 6, respectively. These surface profiles were obtained using a Keyence VHX-2000 digital microscope and indicate a periodic spacing at the micron length scales and a sub-micron height of the laser-induced structures. At this fluence of 1.05 $\mathrm{J} / \mathrm{cm}^{2}$, the structuring starts to emerge even after 1 pulse per spot ( Figure 5a), although the structuring is not consistent across the entire area imaged. After 2 pulses per spot ( Figure 5 b), the laserinterference structuring becomes more evident, while after 6 pulses per spot ( Figure 5c), the quality of the structuring decreases due to significant melting.

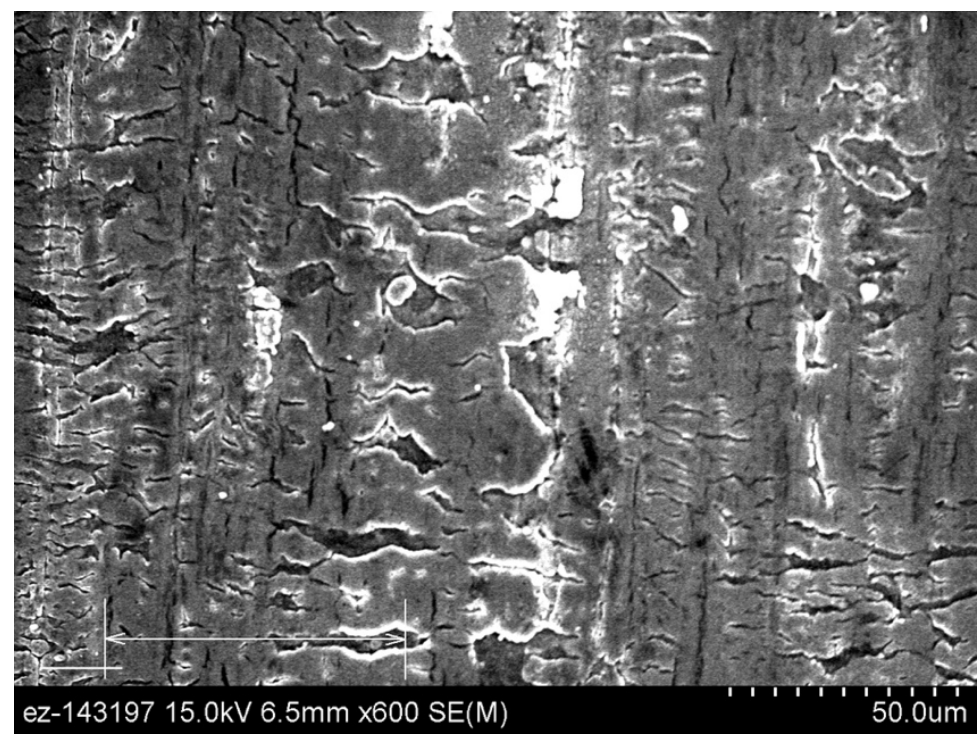

Figure 4. Optical images of as-received Al 5182. 


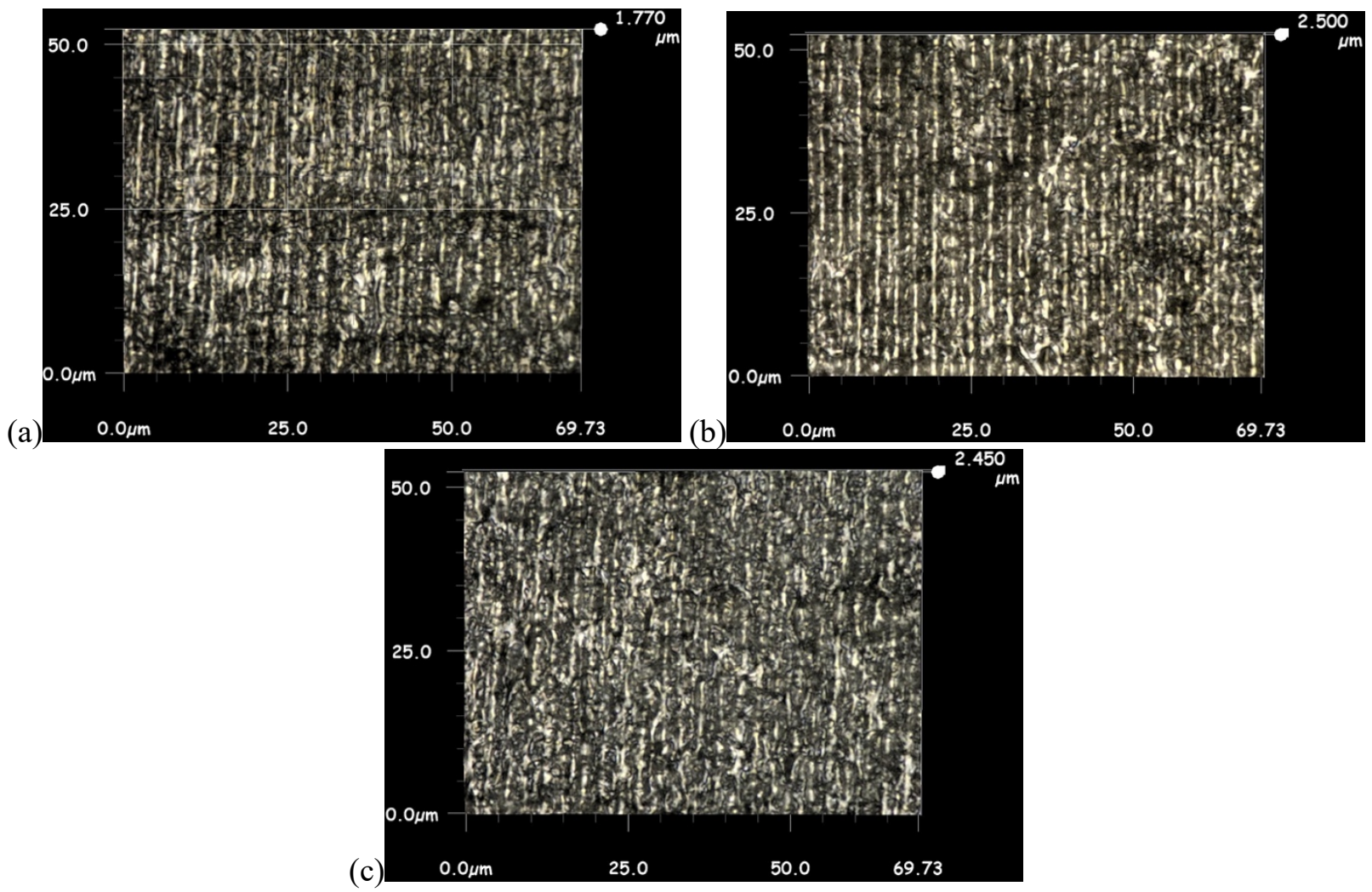

Figure 5. Optical micrograph of laser-interference structured AA5128 surface with a spot size of $4 \mathrm{~mm}$, laser fluence of $1.05 \mathrm{~J} / \mathrm{cm}^{2}$ and the following pulses per spot: (a) 1, (b) 2, and (c) 6 .

\subsection{SURFACE MORPHOLOGY OF AL2024-T3 OBTAINED BY SPOT-BY-SPOT LASER- INTERFERENCE STRUCTURING}

An SEM micrograph of a typical vertical cross-section of as-received material is shown in Figure 6. Aside from the precipiates, which are shown as white irregular shaped spots, the micrograph shows the presence of rounded voids, which appear black. The voids seem to be present throughout the entire sheet thickness.

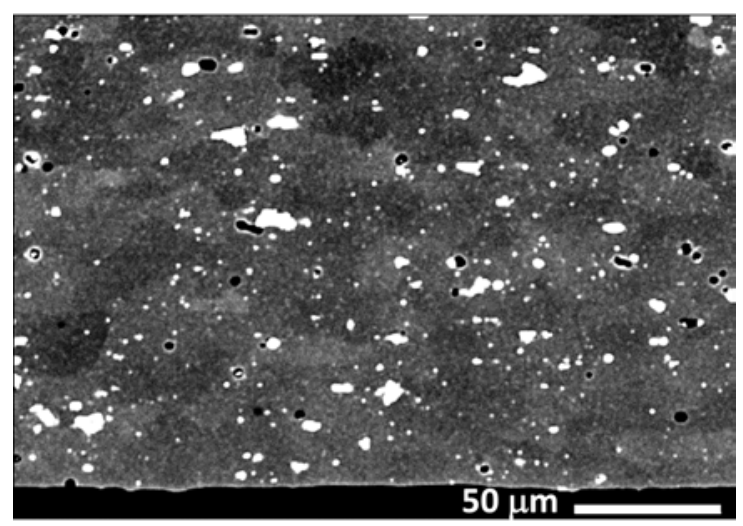


Figure 6. SEM micrograph showing distribution of voids (black, rounded shapes) and precipitates (white, irregular shapes) in vertical cross-section near the top surface.

In Figure 7, top view FE-SEM images are shown for five specimens: the as-received, (unprocessed) specimen and four specimens (A1_2s, A1_4s, A1_6s, and A1_8s) processed with 2, 4, 6, and 8 laser shots-per-spot, respectively, at a spatial period of $\sim 1.7 \mu \mathrm{m}$. The rolling direction is indicated with an arrow for the as-received specimen, which was not laser processed. The grooves, surface depressions, and other surface nonuniformities from prior rolling operations are quite evident. All the other four micrographs were taken with the same specimen orientation, i.e., and rolling direction is identical for all the micrographs. For the 2 shots/spot micrograph, the rolling grooves appeared smoothed out with several pinholes and many shallow crater-like round features. No structuring was present for this condition. In SEM images for the laser-interference structured specimens, blister-like features and holes can be seen. These blister-like features and the tiny holes, which are contributing to roughness and increase in surface area, are likely due to the melting of the wall of the top-most sub-surface voids (Figure 6), exposing them to the ambient. For the 4 shots/spot micrograph, a very shallow structuring appears in a direction almost normal to the rolling direction in most of the image; the structuring is more evident in several large surface depressions. The very shallow structuring is also seen in the 6 shots/spot micrograph, with further smoothing of the rolling grooves and other depressions in the surface, with the exception of the large conglomerate of pinholes in the lower right corner of the image. The 8 shots/spot micrograph exhibit very sharp structuring features. In some areas (e.g., upper left corner) the structuring lines are not continuous with drop-like features aligned in straight lines. In other areas, such as the upperright corner and lower-right corner, the structuring lines are continuous. Due to the relatively rough original surface, the structuring by the laser-interference is expected to be somewhat nonuniform. This observation is consistent with that reported by Sabau et al. (2016) for the interference-induced structuring of A15182, where the variation in the structuring quality included considerations related to the energy absorption and evaporation of surface contaminants, such as lubrication oils, in addition to the optical phenomena related to the non-smooth original surfaces. FE-SEM images show that laser-interference technique "smoothed-out" all the sharp features from the as-received rolling surface (Fig. 1a), minimizing surface defects.

The increased sharpness structuring with the number of shots, $N$, reflects the increase in the structuring depth. Several observations can be made to offer a glimpse in the variation of h with respect $N$. The depth of the first "interference" mound, i.e., after one laser shot, $h_{l}$, depends on the fluence. For subsequent laser shots, the same Marangoni forces as those at previous shots would move less and less molten metal over the uphill slope of the mound; hence the incremental increase in the mound depth, $\Delta h_{i}$, 
for each shot, $i$, would decrease with each subsequent shot. Also, the larger $h_{1}$, the smaller $\Delta h_{i}$ is expected to be, as it more kinetic energy would be required to push the liquid metal uphill for larger mounds. Moreover, if the temperature at the maxima is above the evaporation point, similar amount of evaporation would be expected for each subsequent shot, which would also lead to an increase in the structuring depth. The reflectivity of the surface would change with structuring with each subsequent shot. As the structuring depth would increase, surface reflectivity would decrease, increasing the absorbed energy at the maxima and hence an enhancement in melting and evaporation for the current shot. Without a direct measurement of the structure depth for a fluence of $1.24 \mathrm{~J} / \mathrm{cm}^{2}$, based on data on Table $2 \mathrm{~h}_{1}$ may be considered to be smaller than $0.29 \mu \mathrm{m}$ (or even $0.05 \mu \mathrm{m}$ ). This small depth may explain why the structuring is not evident in the SEM micrographs for the 2 shots/spot case.
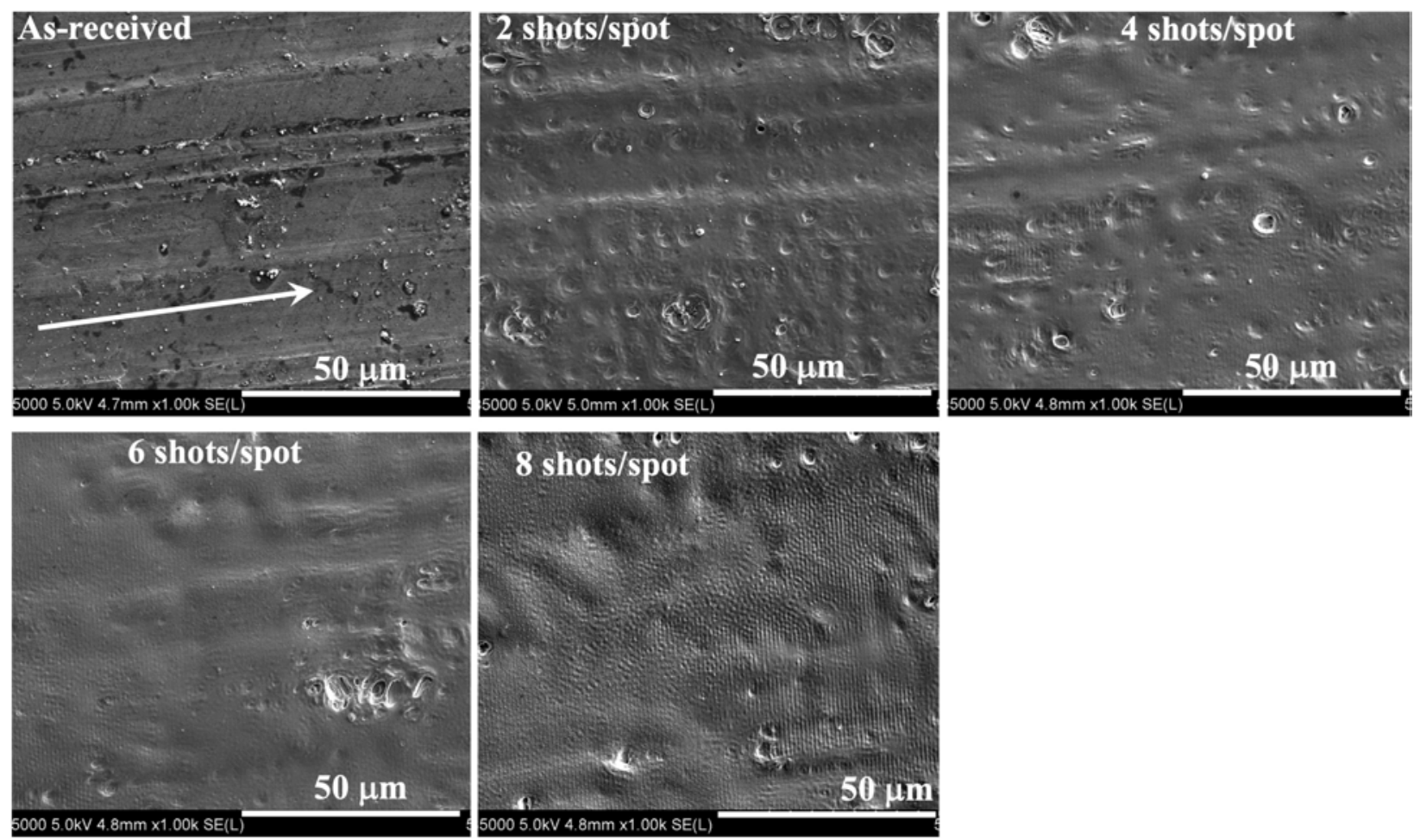

Figure 7. Top view FESEM images for the as-received condition and four laser-structured conditions at a spatial period of $1.7 \mu \mathrm{m}$ with 2, 4, 6, and 8 laser shots/spot (A0, A1_2s, A1_4s, A1_6s, and A1_8s).

In Figure 8, top view FE-SEM images are shown for four laser-structured specimens (A2_2s, A2_4s, A2_6s, and A2_8s) processed with 2, 4, 6, and 8 laser shots-per-spot, respectively; at a spatial period of $\sim 0.6 \mu \mathrm{m}$. The grooves, surface depressions, and other surface nonuniformities from prior rolling 
operations are quite evident; and at the high-magnification of 50k used some of the areas were out of focus. For the 2 shots/spot micrograph (Figure 8a), the rolling groove, located on the top area of the image, appeared smoothed out with several pinholes and many shallow crater-like round features; this is similar to the SEM image for a periodicity of $1.7 \mu \mathrm{m}$ (Figure 7). However, some very faint structuring was present for this condition. For the 4 shots/spot micrograph, a very shallow structuring was found to cover a wide area. The shallow but sharper structuring is seen in the 6 shots/spot micrograph. The structuring ridges were narrower than that of the depressions between them. The structuring ridges appear to connect to the adjacent ridges across the structuring depressions at $\sim 3 \mu \mathrm{m}$ lengths. The 8 shots/spot micrograph exhibited smoother and wider ridges than those seen for the 6 shots/spot. In summary, the similar shallow laser-structuring is exhibited for a spatial period of $\sim 0.6 \mu \mathrm{m}$ as that for the spatial period of $\sim 1.7 \mu \mathrm{m}$. This spatial period of $\sim 0.6 \mu \mathrm{m}$ is smaller than the minimum spatial period $d_{\min } \sim 1.3 \mu \mathrm{m}$, which was estimated for A12024 in Section 2.3 for an 8 ns laser pulse and a thermal diffusion length of $L_{t h} \sim 0.65 \mu \mathrm{m}$. Understanding the formation of the structuring pattern at this smaller spatial period would require detailed modeling of the energy transport (Lasagni et al., 2007, D’Alessandria et al., 2008, and Bieda et al., 2010), by including additional effects due to alloy solidification over a wide solidification temperature range and fluid dynamics effects that can affect the melt-pool shape during laser processing (Sabau et al. 2020). 

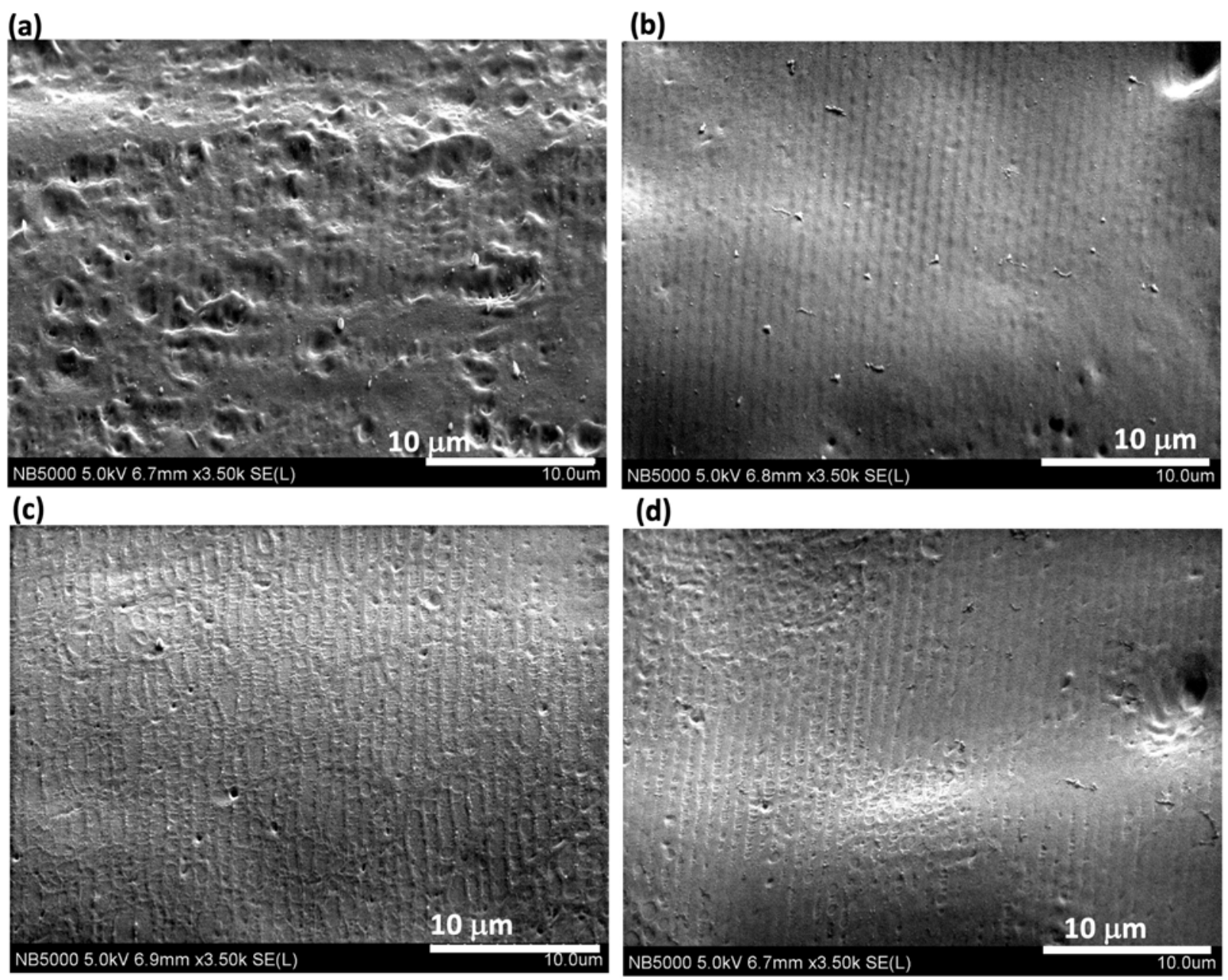

Figure 8. Top view FESEM images for laser-structured specimens at a spatial period of $\sim 0.6 \mu \mathrm{m}$ with $2,4,6$, and 8 laser shots/spot (A2_2s, A2_4s, A2_6s, and A2_8s).

\subsection{PROFILOMETRY OF AL2024-T3 SURFACES OBTAINED BY SPOT-BY-SPOT LASER- INTERFERENCE STRUCTURING}

The 3D height distributions of typical surfaces in the as-received (unprocessed condition) and after laserinterference processing with 8 shots/spot for at a spatial period of $\sim 1.7 \mu \mathrm{m}$ are shown in Figure 9 . For the laser-structure specimens, the profiled area was located approximately $1 \mathrm{~mm}$ away from the center of each laser spot. The specimen orientation with respect to the rolling direction was identical for all three profiles shown in Figure 9. The structuring induced by the laser-interference is evidenced by the very fine striations normal to the rolling direction, i.e., along the $174 \mu \mathrm{m}$ long axis (Figure $9 \mathrm{~b}$ and $\mathrm{c}$ ). The $\mathrm{R}_{\mathrm{a}}, \mathrm{R}_{\mathrm{q}}$, and $\mathrm{R}_{\mathrm{t}}$ are shown in 
Table 4. The roughness was measured for the sake of completeness, not as a quantifying measure of "smoothing out of the sharp features." The as-received Al sheet surface was found to have an RMS roughness of $0.462 \mu \mathrm{m}$ and maximum height of features of $4.61 \mu \mathrm{m}$. An increase in all surface roughness metrics can be observed for the laser structured specimens with respect to the those measured for the asreceived specimens, i.e., without any laser structuring. Due to the small resolution (vertical $<0.1 \mathrm{~nm}$; lateral $\sim 130 \mathrm{~nm}$ ) the differences reported can be considered statistically significant between the asreceived and laser structured specimens. The mean deviations of the roughness profile, $\mathrm{R}_{\mathrm{a}}$ and $\mathrm{R}_{\mathrm{q}}$, were found to increase for the laser structured surfaces by approximately $2-5 \%$ and $\sim 9 \%$, respectively. The increase in $R_{a}$ and $R_{q}$ is thought to be attributed to the additional roughness created by periodic laserinterference structuring, on top of the already "grooved" Al sheet from rolling operations and the exposure of the near sub-surface voids.
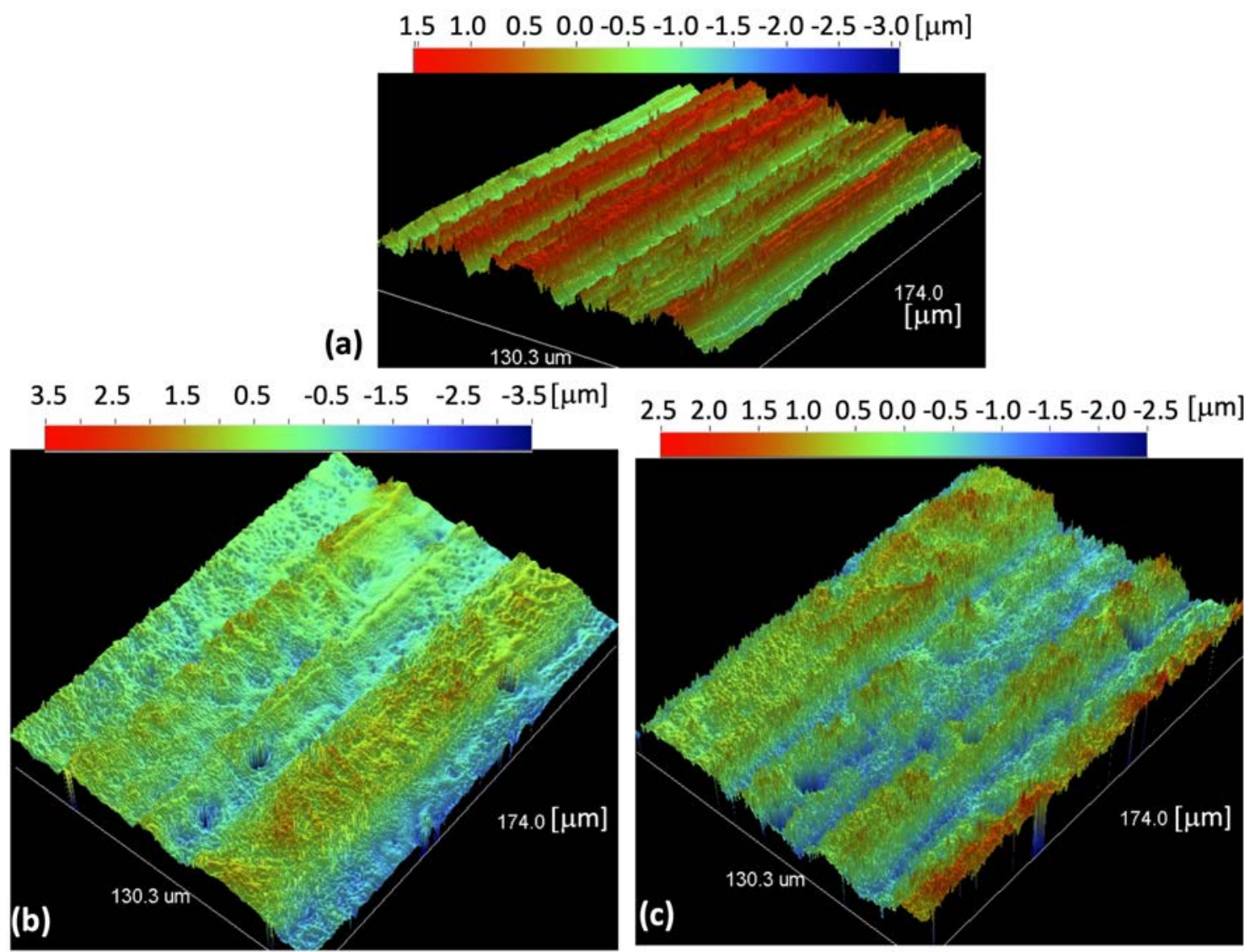

Figure 9. Surface profiles for: (a) as-received specimen (A0) and (b, c) 8 shots/spot specimen A1_8s at a spatial period of $\sim 1.7 \mu \mathrm{m}$. 
Table 4. Surface roughness for specimen A1_8s at a spatial period of $\sim 1.7 \mu \mathrm{m}$

\begin{tabular}{|c|c|c|c|}
\hline Specimen & $\mathrm{R}_{\mathrm{a}}[\mathrm{nm}]$ & $\mathrm{R}_{\mathrm{q}}[\mathrm{nm}]$ & $\mathrm{R}_{\mathrm{t}}[\mu \mathrm{m}]$ \\
\hline $\mathrm{A} 0$ & 462 & 545 & 4.61 \\
\hline A1_8s & 472 & 593 & 6.75 \\
\hline A1_8s & 487 & 597 & 7.36 \\
\hline
\end{tabular}

*as-received, without any laser processing

\subsection{PROFILOMETRY OF AL2024-T3 SURFACES OBTAINED BY RASTER LASER- INTERFERENCE STRUCTURING}

The profiling surface covered an area of $130 \times 174 \mu \mathrm{m}$. The 3D height distributions of typical surfaces in the as-received, and unprocessed condition and after laser-interference processing are shown in Figure 10. The surface profiles for the as-received surfaces are shown in Figure 10a and Figure 10b for two specimens. The first specimen contains areas that were laser processed with scanning speeds of 2 and $4 \mathrm{~mm} / \mathrm{s}$ while the second specimen contains areas processed with scanning speeds of 6 and $8 \mathrm{~mm} / \mathrm{s}$. The surface profiles are shown in Figure $10 \mathrm{c}$, d, e, and f for all laser scanning speeds of 2, 4, 6, and $8 \mathrm{~mm} / \mathrm{s}$, respectively. The laser scan direction was identical to that of the rolling direction.

Optical images obtained with the profilometer are shown for the sake of completion in Figure 11 while high-resolution SEM images for these laser processing conditions are recently presented in Meyer et al. [19]. Due to the height variation and optical limitations, some regions would appear out of focus in the optical micrographs, however, the information from the profilometry and its corresponding optical image are complimentary. For example, the surface defects intrinsic to the prior rolling operations and interference-induced structuring is more evident in the optical micrographs than in the 2D profilometry images. For better detail, optical images obtained with the profilometer are shown only for a quarter of the profiling surface, covering an area of $65 \times 87 \mu \mathrm{m}$. The exact quarter where each optical micrograph was taken is indicated with two letters that indicate the position of the optically imaged area in the overall profiling area, namely LL, LR, TL, and TR for lower-left, lower-right, top-left, and top-right, respectively. As evidenced by Figures 1a, 2a, 1b, and 2b, the as-received and unprocessed aluminum surfaces are not smooth at all, exhibiting grooves in the horizontal direction from the rolling operation, quite a few microcracks, and pin-holes. The grooves are evidenced by horizontal dark stripes while the pinholes by round, dark-like features. The microcracks are evidenced by the jagged like dark-lines between the rolling grooves and are more visible in the optical micrographs (Figure 11a and b) than in the profilometry images. 

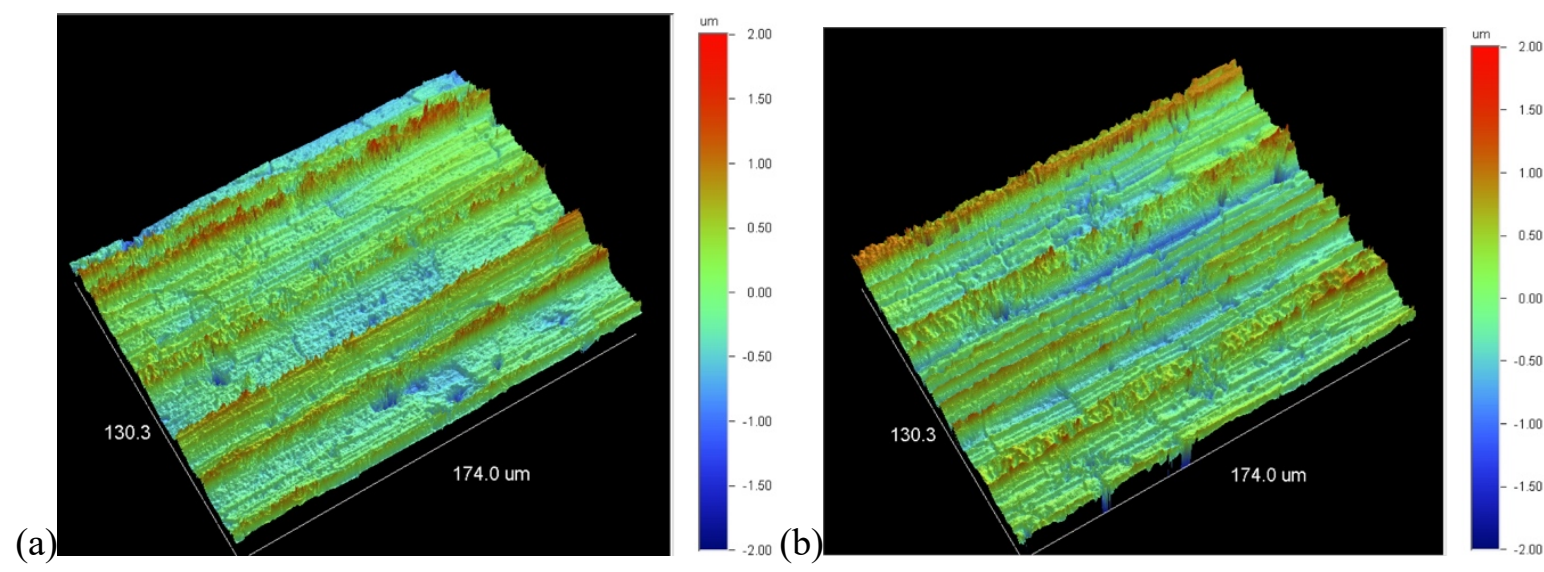

(c)
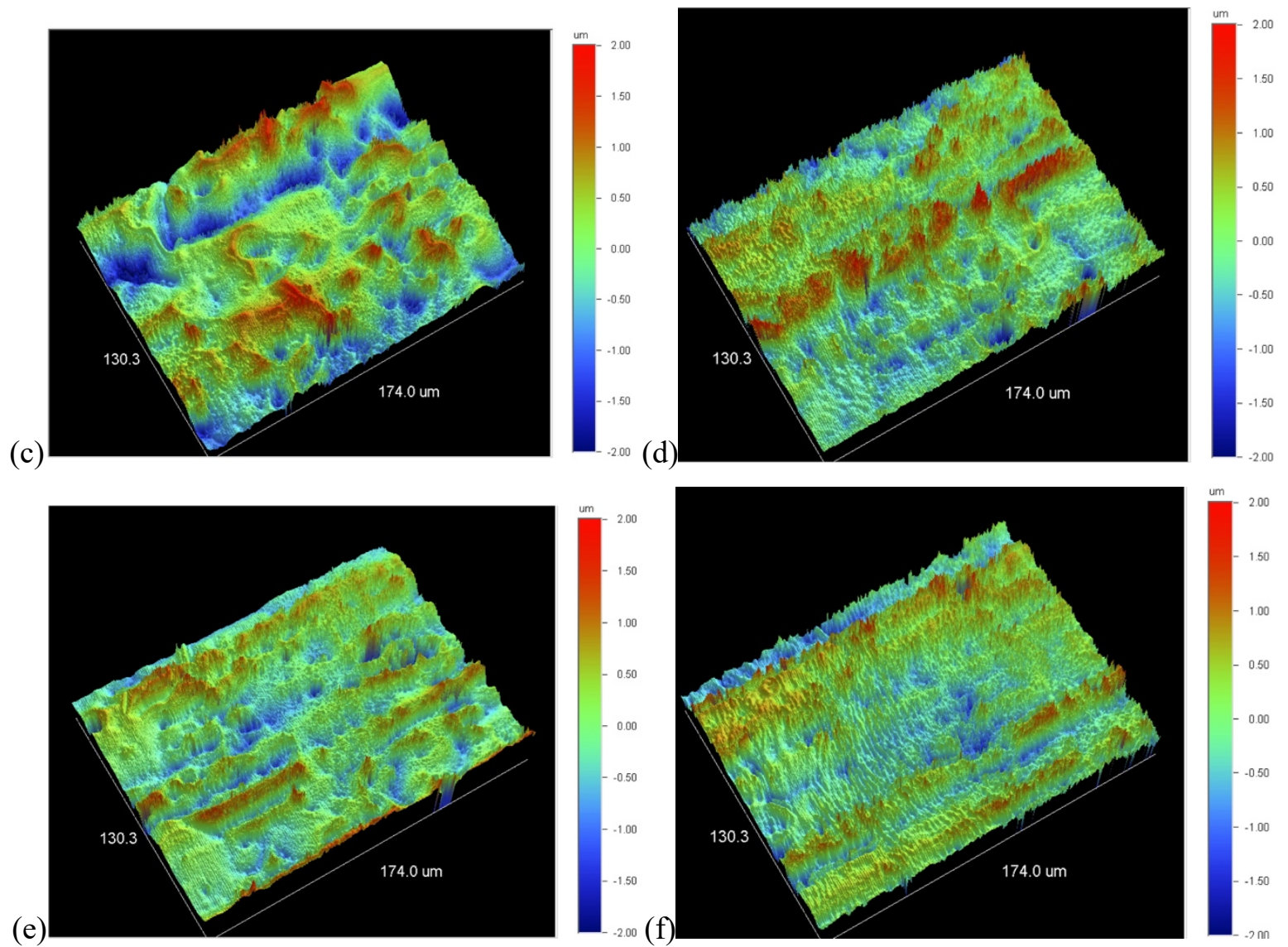

Figure 10. Surface profiles at a laser fluence of $F 1=1.238 \mathrm{~J} / \mathrm{cm}^{2}$ per pulse for $(a, b)$ the as-received specimens and in typical centerlines of a laser scan for raster speeds of: (c) $2 \mathrm{~mm} / \mathrm{s}$, (d) $4 \mathrm{~mm} / \mathrm{s}$, (e) $6 \mathrm{~mm} / \mathrm{s}$, and (f) 8 $\mathbf{m m} / \mathbf{s}$. 
(a)

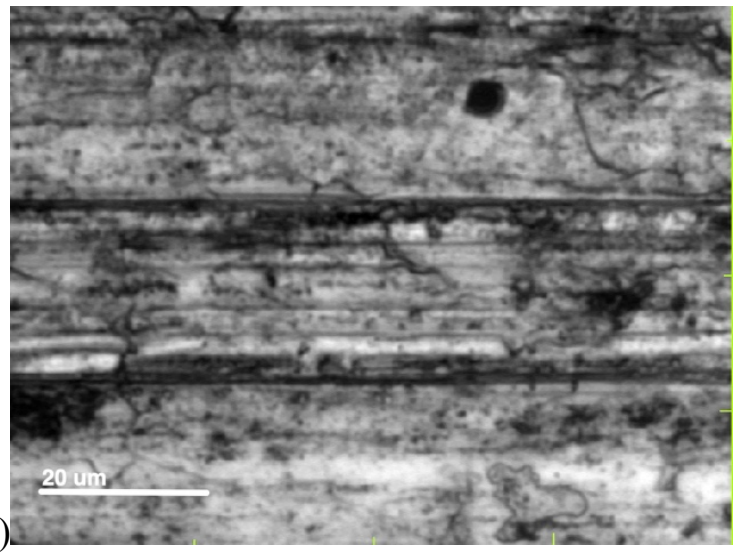

(c)

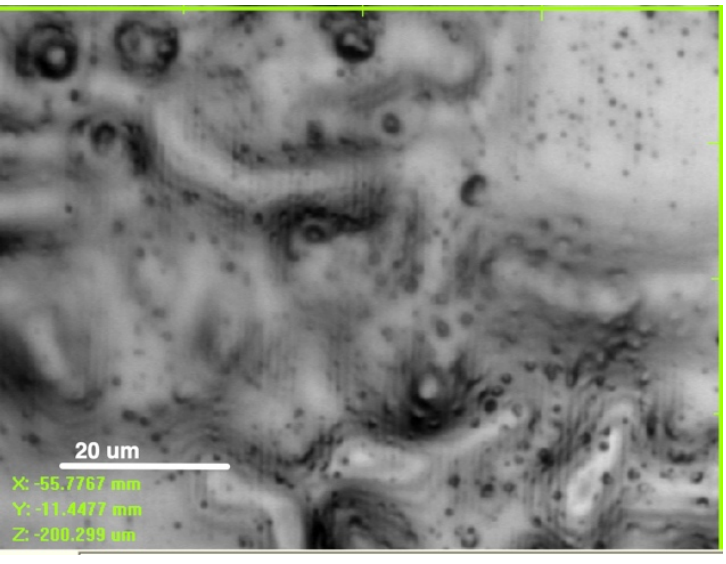

(e)

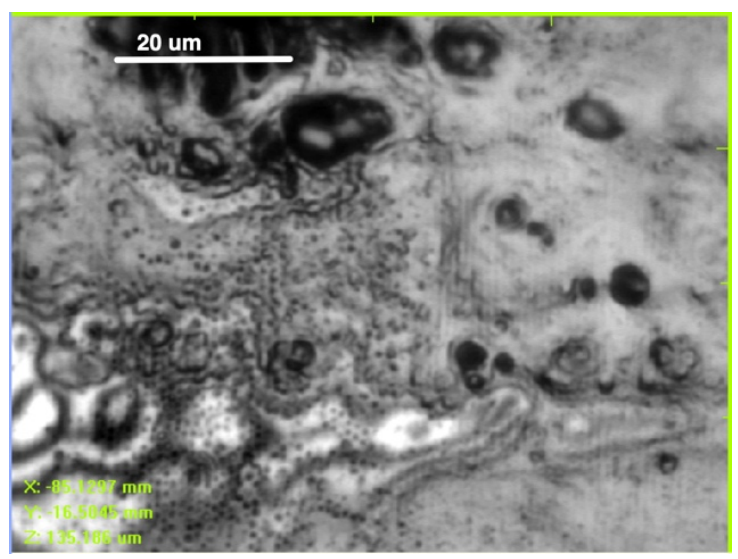

(b)

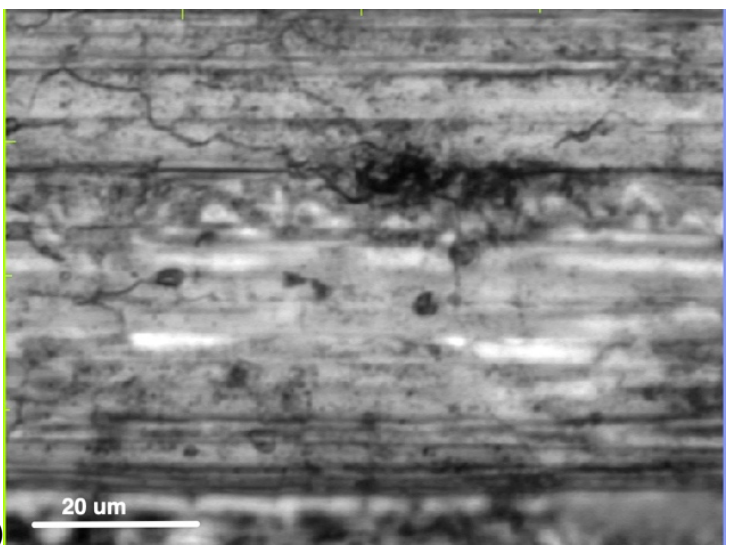

(d)
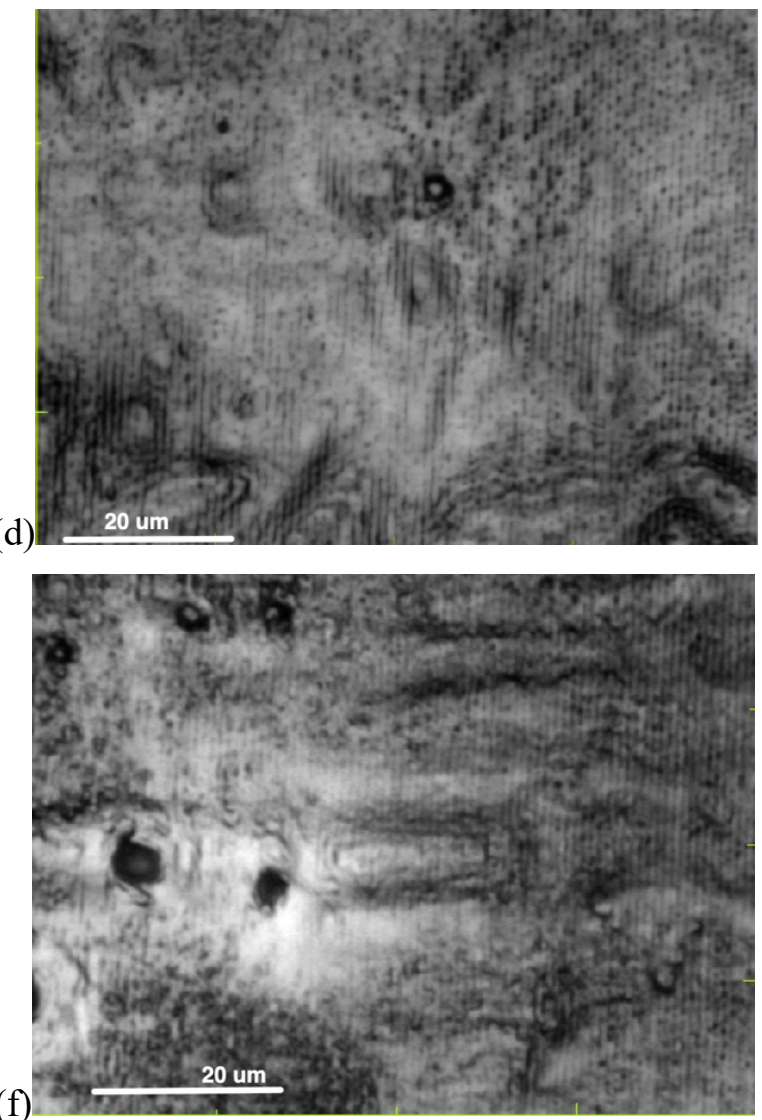

Figure 11. Optical micrographs acquired by the profilometer. The corresponding location of the quarter area to the profilometry images shown in Figure 10 is indicated in parenthesis: $(a, b)$ the as-received specimens (TL, BL) and in the typical centerline of a laser scan for raster speeds of: (c) $2 \mathrm{~mm} / \mathrm{s}$ (BL), (d) $4 \mathrm{~mm} / \mathrm{s}$ (TR), (e) $6 \mathrm{~mm} / \mathrm{s}(B L)$, and (f) $8 \mathrm{~mm} / \mathrm{s}$ (TL). Laser fluence was $F_{1}=1.238 \mathrm{~J} / \mathrm{cm}^{2}$ per pulse.

The structuring induced by the laser-interference is evidenced by the very fine striations in the vertical direction, i.e., normal to the rolling direction (Figure $9 \mathrm{c}$ and e for the 3D profilometry; and Figure 10d and $\mathrm{f}$ in the optical micrographs). In spite of the relatively rough original surface, the structuring by the laser- 
interference is thus shown to be pretty robust. This observation is consistent with that reported by (Sabau, Greer, et al. 2016) for the interference-induced structuring of Al5182 aluminum alloy, where the variation in the structuring quality was explained by the considerations related to the energy absorption and evaporation of surface contaminants, such as lubrication oils, in addition to the optical phenomena related to the non-smooth original surfaces.

\subsection{RESULTS FOR ROUGHNESS PARAMETERS}

The average roughness, or arithmetical mean deviation of the roughness profile, $R_{a}$, root-mean-square deviation of the roughness profile, $R_{\mathrm{q}}$, maximum roughness, $\mathrm{R}_{\mathrm{z}}$, and maximum height of the roughness profile, $\mathrm{R}_{\mathrm{t}}$, are shown in Table 5. As the height of the laser-interference structuring is expected to be less than 1.5 microns (Lasagni et al. 2007)while the $R_{z}$ and $R_{t}$ are on the order of 4 to $5 \mu m$, the $R_{z}$ and $R_{t}$ are expected to be the least affected by the laser structuring. An increase in all surface roughness metrics can be observed between the indicators measured for the laser structured specimens with respect to the indicators measured for the as-received specimens, i.e., without any laser structuring. The increase in the surface roughness indicators with respect to that of the unprocessed condition, i.e., $d R[\%]=$ $100\left(R / R_{O}-1\right)$, is shown in

Table 6. The mean deviations of the roughness profile $\left(R_{a}\right.$ and $\left.R_{q}\right)$ were found to increase for all of the laser structured conditions by approximately 20 to $50 \%$.

Table 5. The surface roughness at a fluence of $F_{1}=1.238 \mathrm{~J} / \mathrm{cm}^{2}$ per pulse

\begin{tabular}{|c|c|c|c|c|}
\hline $\begin{array}{c}\text { Raster speed } \\
{[\mathrm{mm} / \mathrm{s}]}\end{array}$ & $\begin{array}{c}\mathrm{R}_{\mathrm{a}} \\
{[\mathrm{nm}]}\end{array}$ & $\begin{array}{c}\mathrm{R}_{\mathrm{q}} \\
{[\mathrm{nm}]}\end{array}$ & $\begin{array}{c}\mathrm{R}_{\mathrm{z}} \\
{[\mu \mathrm{m}]}\end{array}$ & $\begin{array}{c}\mathrm{R}_{\mathrm{t}} \\
{[\mu \mathrm{m}]}\end{array}$ \\
\hline$* 0$ & 311.9 & 394 & 4.3 & 4.98 \\
\hline 2 & 483.5 & 611.1 & 4.61 & 5.12 \\
\hline 4 & 382.3 & 511.6 & 5.29 & 5.81 \\
\hline$* 0$ & 282.5 & 358.9 & 4 & 4.87 \\
\hline 6 & 334.6 & 439.9 & 5.79 & 7.45 \\
\hline 8 & 359.4 & 442 & 5.26 & 6.15 \\
\hline
\end{tabular}

*as-received, without any laser processing 
Table 6. The percentage increase in surface roughness metrics with respect to those for the asreceived condition at a fluence of $F 1=1.238 \mathrm{~J} / \mathrm{cm}^{2}$ per pulse

\begin{tabular}{|c|c|c|c|c|}
\hline $\begin{array}{c}\text { Raster speed } \\
{[\mathrm{mm} / \mathrm{s}]}\end{array}$ & $\begin{array}{c}\mathrm{dR}_{\mathrm{a}} \\
{[\%]}\end{array}$ & $\begin{array}{c}\mathrm{dR}_{\mathrm{q}} \\
{[\%]}\end{array}$ & $\begin{array}{c}\mathrm{dR}_{\mathrm{z}} \\
{[\%]}\end{array}$ & $\begin{array}{c}\mathrm{dR}_{\mathrm{t}} \\
{[\%]}\end{array}$ \\
\hline 2 & 55 & 55 & 7 & 2 \\
\hline 4 & 22 & 29 & 23 & 16 \\
\hline 6 & 18 & 22 & 44 & 52 \\
\hline 8 & 27 & 23 & 31 & 26 \\
\hline
\end{tabular}

*as-received, without any laser processing

\subsection{RESULTS FOR SURFACE AREA OF LASER STRUCTURED SPECIMENS}

As evidenced by the profilometry data, LIS increases the surface area for adhesion. An attempt is made here to quantify the "roughness factor," $r_{L I S}$, to use same the terminology introduced for coating adhesion (Wenzel 1936). $r_{L I S}$ is thus defined as the ratio of the LIS solid surface area to the original and unprocessed surface area. For one laser pulse, the expected surface morphology is shown in Figure 12 and would consist in alternating depressions unto the as-received surface (in regions of maxima interference laser power) and mounds over the as-received surface due to the solidification of the migrated liquid metal (from maxima power regions). These alternating depression-and-mound structures are generated by the freezing of the nanosecond Marangoni-induced metal flow as indicated by (Lasagni et al. 2007) and (Sabau, Greer, et al. 2016). For the raster mode, the surface is exposed to multiple laser pulses, as indicated by the number of equivalent pulses, $N_{P}$, in Section 3.2. As the regions of maxima power created by the interference may not be superimposed for successive laser pulses, and some of the mounds formed at previous pulses maybe partially remelted and liquid metal would be moved partially to the depressions and partially to the mounds. Thus, the resulting surface morphology may be more complex than that illustrated in Figure 12. In order to estimate the surface area after LIS, the wetted perimeter of the depression-and-mound LIS in the direction normal to the structuring direction has to be evaluated. The following considerations and assumptions can be made based on experimental evidence of the LIS geometry from (Lasagni et al. 2007) and Figure 11, as illustrated in Figure 12: (a) per each periodicity interval, $\mathrm{d}$, the LIS profile can be approximated by a trapezoid with a lower base, $b$, and top base, a, and height, $\mathrm{h}$; for low fluences, there would be two mounds per periodicity as the mounds, which grow from each of the neighboring depressions, do not merge; (b) the trapezoid shape is considered to be 
weakly dependent on the fluence, as the LIS induced depressions and mounds are based on

migrating/spreading of the Marangoni-induced liquid metal and freezing it in place after the 2-3 ns pulse, hence the ratio a/b is considered to be fixed at 0.5 ; (c) $h(F, U)$ varies with fluence and raster speed $\mathrm{U}$ (through $N_{P}$ ); (d) the base size, b, was considered to be fixed at $\sim 0.5 \mathrm{~d}$ (as it can be inferred from Figure

11). To summarize, the roughness factor can be calculated, as:

$$
r_{L I S}(F, U)=1+\frac{b}{d}\left[2 \sqrt{\frac{1}{4}\left(\frac{a}{b}-1\right)^{2}+\left(\frac{h(F, U)}{b}\right)^{2}}+\frac{a}{b}-1\right],
$$

with $\frac{a}{b}=0.5$ and $\frac{b}{d}=0.5$. The height of the first "interference" mound, i.e., after one laser pulse, $h_{l}$, depends on the fluence. For one laser pulse, the $h_{1}$ was considered to be 0.05 and $1.2 \mu \mathrm{m}$ for fluences of 1.24 and $1.78 \mathrm{~J} / \mathrm{cm}^{2}$, respectively, as indicated by (Lasagni et al. 2007) for pure Al. Several observations can be made to offer a glimpse in the variation of $\mathrm{h}$ with respect $N_{P}$ (and consequently with $\mathrm{U}$ ). For subsequent laser pulses, the same Marangoni forces as those at previous pulses would move less and less molten metal over the uphill slope of the mound; hence the incremental increase in the mound height, $\Delta \mathrm{h}_{\mathrm{i}}$, for each pulse, $\mathrm{i}$, would decrease with each subsequent pulse (i.e., $\Delta h_{i+1}<\Delta h_{i}$ ). Also, the larger $\mathrm{h}_{1}$, the smaller $\Delta \mathrm{h}_{\mathrm{i}}$ is expected to be, as it more kinetic energy would be required to push the liquid metal uphill for larger mounds. To qualitatively estimate $h(F, U)$, it is assumed that $\Delta h_{i+1}=\beta(F) \Delta h_{i}$ and that $\beta$ is 0.5 and 0.8 for fluences of $1.78 \mathrm{~J} / \mathrm{cm}^{2}\left(h_{l}=1.2 \mu \mathrm{m}\right)$ and $1.24 \mathrm{~J} / \mathrm{cm}^{2}\left(h_{l}=0.05 \mu \mathrm{m}\right)$, respectively. With these assumptions, $h(F, U)$, can be calculated, as:

$$
h(F, U)=h_{1}(F) \frac{1-\beta(F)^{N_{P}(U)}}{1-\beta(F)}
$$

The calculated values for $h(F, U)$ and $r_{L I S}$ are shown in Table 7. For comparison, $r_{L I S}$ for the first pulse was estimated to be 1.014 and 2.18 for fluences 1.24 and $1.78 \mathrm{~J} / \mathrm{cm}^{2}$, respectively. As expected, $h$ and $r_{L I S}$ exhibited weaker variation with $N_{P}$ (and U) especially at medium $/$ high fluences (e.g., $1.78 \mathrm{~J} / \mathrm{cm}^{2}$ with $h_{l}=1.2 \mu \mathrm{m}$ ) than those at low fluences (e.g., $1.24 \mathrm{~J} / \mathrm{cm}^{2}$ with $\left.h_{l}=0.05 \mu \mathrm{m}\right)$. The U-dependence of $r_{L I S}$ is weaker at lower $\mathrm{U}$ values ( 4 to $6 \mathrm{~mm} / \mathrm{s}$ ) and weaker at the larger fluence of $1.78 \mathrm{~J} / \mathrm{cm}^{2} . r_{L I S}$ is smallest at highest speed $(10 \mathrm{~mm} / \mathrm{s})$. This qualitative data will be used further on to understand the effect of raster speed on the coating adhesion. 


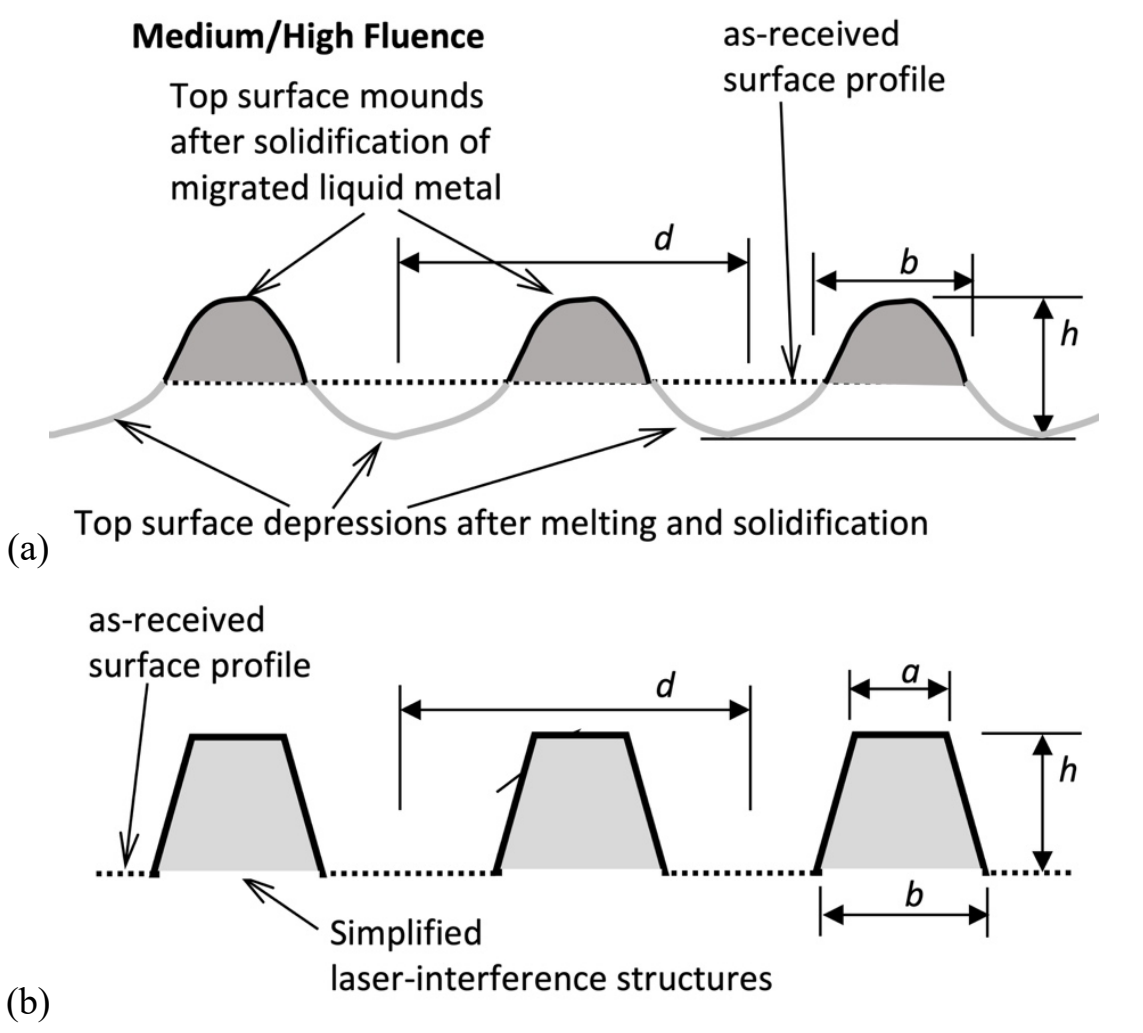

Figure 12. Schematic of: (a) LIS surface topology and (b) simplified surface profile.

Table 7. Estimated LIS height, $h$, and roughness factor, $r_{L I S}$, for several raster speeds

\begin{tabular}{|c|c|c|c|c|c|c|}
\hline$F_{1}\left[\mathrm{~J} / \mathrm{cm}^{2}\right]$ & \multicolumn{3}{|c|}{1.238} & \multicolumn{3}{c|}{1.782} \\
\hline$U[\mathrm{~mm} / \mathrm{s}]$ & $N_{P}$ & $h[\mathrm{um}]$ & $r_{\text {LIS }}$ & $N_{P}$ & $h[\mathrm{um}]$ & $r_{\text {LIS }}$ \\
\hline 4 & 15 & 0.24 & 1.26 & 13 & 2.40 & 3.58 \\
\hline 6 & 10 & 0.22 & 1.23 & 8 & 2.39 & 3.57 \\
\hline 8 & 7 & 0.20 & 1.18 & 6 & 2.36 & 3.54 \\
\hline 10 & 6 & 0.18 & 1.16 & 5 & 2.33 & 3.50 \\
\hline
\end{tabular}

\subsection{VERTICAL SUB-SURFACE MICROSTRUCTURE OF AL2024-T3 OBTAINED BY SPOT- BY-SPOT LASER-INTERFERENCE STRUCTURING}

The STEM characterization is presented in vertical cross-sections to investigate the laser-interference effects on the sub-surface microstructure. The STEM Z-contrast images for as-received and laserinterference processed specimens are shown in Figure 13. The structuring is evident for the 8-shot specimens, as shown in details of the transmission electron microscopy (TEM) foil liftout were shown for 
the 8-shot specimen in (Sabau, Meyer, and Leonard 2020). Due to the variation in structuring, such as discontinuity in structuring lines (Figure 11), and the nm thickness of TEM specimens, this type STEM characterization is not appropriate to investigate the surface structuring seen in these specimens.

The STEM images of the as received material reveal the presence of typical precipitates in A12024 alloy (Brown and Kobayashi 2001); (Ryan and Prangnell 2008) and show that precipitates are uniformly distributed through the thickness of the as-received specimen, without any laser processing (Figure 13a). For the laser-structured specimens, the STEM images shown in Figure 13b and Figure 13c indicate that the laser-interference processing affected the top 500 to $800 \mathrm{~nm}$ of the specimen surface. Within that first 500-800 nm, the $\mathrm{Al}_{2} \mathrm{Cu}$ precipitates were simply not present at all for the 2-shot laser specimen, while these precipitates are expected in the un-processed specimen as evidenced by typical STEM images, such as the one shown in Figure 13a. For the 8-shot specimen, a relatively large microstructure feature can be observed, in an otherwise precipitate free region. Both the 2-shot (A1_2s) and 8-shot (A1_8s) samples showed microstructure details that suggested that the nano-scale precipitates observed in the as-received specimen had in fact been dissolved into the matrix material in the top $500 \mathrm{~nm}$ region of the laser processed samples. Evidence of this proposed laser processing induced precipitate dissolution, observed in the STEM micrographs as well as EDS X-ray maps, are discussed further below.

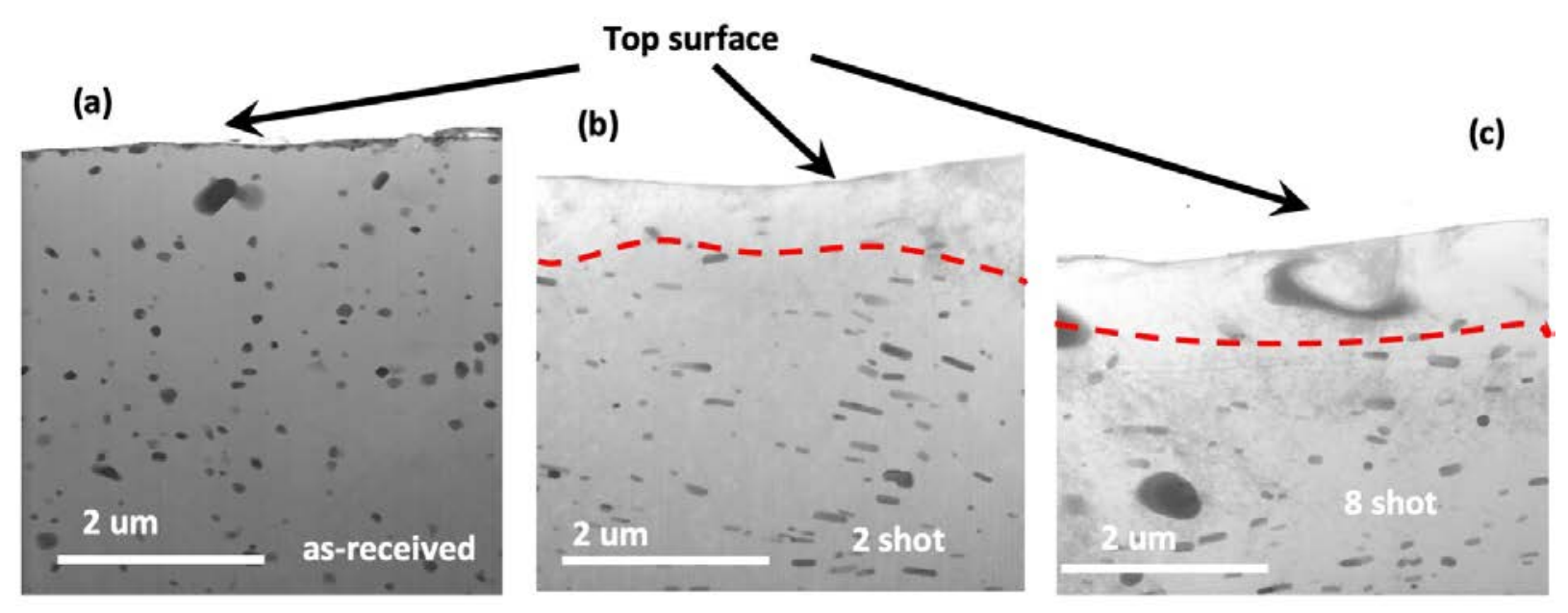

Figure 13. STEM micrographs showing distribution of precipitates (black or dark gray colors) in vertical cross-sections near the top surface for A12024-T3 specimens: (a) as-received and laser-interference processed with (b) 2 shots-per-spot (A1_2s) and (c) 8 shots-per-spot (A1_8s). 


\subsection{ELEMENTAL MAPS IN SUBSURFACE CROSS-SECTIONS OF AL 2024 OBTAINED BY SPOT-BY-SPOT LASER-INTERFERENCE STRUCTURING}

The elemental maps for $\mathrm{Al}, \mathrm{Cu}, \mathrm{Fe}, \mathrm{Mg}, \mathrm{Mn}$, and $\mathrm{O}$ are shown in Figure 14 and Figure 15 for the asreceived specimen and the 8-shot laser-structured specimen, respectively. The imaging was obtained with the high-angle annular dark field (HAADF) technique. The elemental maps can be used to identify CuFerich precipitates (larger size) and Mn-rich precipitates (smaller in size). The elemental maps for the laserstructured specimen with 8-shot per spot were sized such that the scale marker has the same length as that for the as-received specimen. The elemental maps for the 8-shot sample, A1_8s (Fig. 6) were obtained for the first 1 micron, trying to image the detail on a large, curved feature observed in STEM images (Figure 13c). The elemental maps show that this feature contains $\mathrm{Cu}$ and $\mathrm{Fe}$ and that it is not $\mathrm{Mn}$ rich as most of the precipitates observed in the as-received specimen. The $\mathrm{Cu}$ and $\mathrm{Fe}$ content are also smaller than those in the large precipitate evidenced in the EDS maps for the as-received specimen.

For the as-received sample (Figure 14), the average matrix composition (without precipitates included) in wt.\%, as determined by EDS, was found to be $91.3 \% \mathrm{Al}, 6.0 \% \mathrm{Cu}, 0.1 \% \mathrm{Mn}, 1.7 \% \mathrm{Mg}$. The largest particle measuring $400 \mathrm{~nm}$ long and $200 \mathrm{~nm}$ wide, labeled 1 , was found to have a nominal elemental composition in wt. $\%$ of $45 \% \mathrm{Al}, 40 \% \mathrm{Cu}, 3.5 \% \mathrm{Mn}$ and $11 \% \mathrm{Fe}$. Smaller precipitates, examples labeled 2 and 3, were determined to have a nominal elemental composition of $69 \% \mathrm{Al}, 15 \% \mathrm{Cu}, 14.7 \% \mathrm{Mn}$ with less than $1 \%$ of both $\mathrm{Mg}$ and Si. Also observed in the EDS analysis was an increase in the $\mathrm{Mg}$ and $\mathrm{O}$ signal from the surface of the specimen which was also found by XPS analysis and discussed in the following sections. 

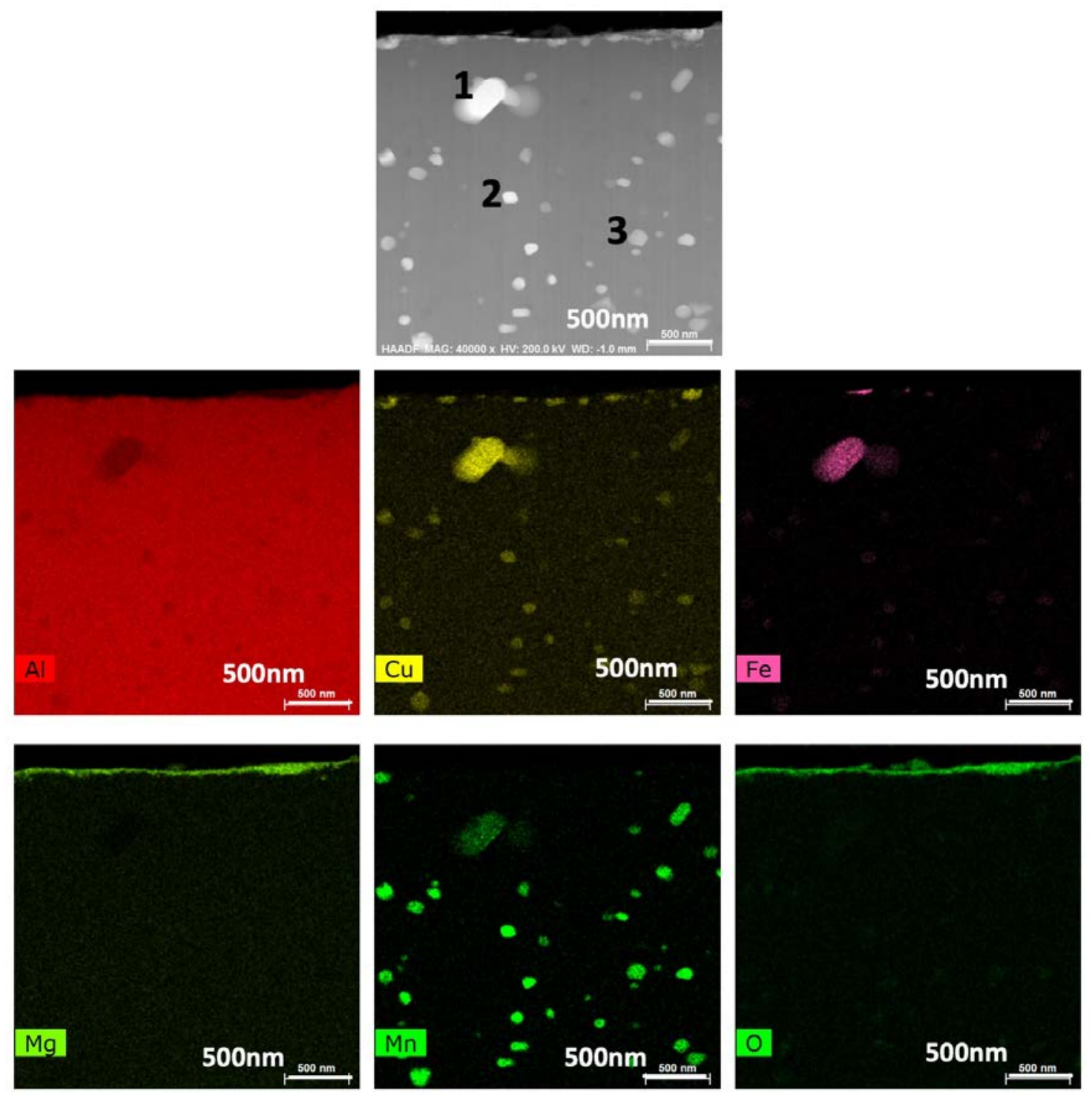

Figure 14. Maps for HAADF STEM image for elements $\mathrm{Al}, \mathrm{Cu}, \mathrm{Fe}, \mathrm{Mg}, \mathrm{Mn}$, and O. Data for the vertical cross-section as-received specimen (without any laser processing).

To compare the elemental composition of the matrix in as-received (A0) and as processed samples EDS data from the 8-shot sample (A1_8s) was acquired in the region indicated in Figure 15. Like the 2-shot sample, EDS analysis was done in a region $\sim 1$ um below the surface. The average as-processed 8-shot matrix contained 90 wt.\% Al, 6.9 wt.\% Cu, $0.3 \mathrm{Wt} . \% \mathrm{Mn}, 2$ wt.\% Mg and 1 wt.\% O. But the STEM HAADF micrograph of Figure 15 also clearly shows a change in $2^{\text {nd }}$ phase particle morphology after laser processing. Second phase particles common in the 2024 alloy have already been discussed and identified above. The morphology of these second phases in the baseline material consist of faceted and elongated structures on the nano and micro scale, but all have a well delineated boundary at the particle/matrix 
interface. In the first 1 um below the surface of 8 -shot as-processed 2024 samples $2^{\text {nd }}$ phase particles exhibited a blurred interfacial region between the particle/matrix interface. At higher magnifications this laser processed particle/matrix boundary was observed to have a dendritic structure and may indicate dissolution of the very large second phase particles. To further explore the large $2^{\text {nd }}$ phase particle dissolution as a result of laser processing hypothesis EDS of the bulk of the $2^{\text {nd }}$ phase particle and in the interfacial boundary region of the particle was compared. The large $2^{\text {nd }}$ phase particle had a bulk composition of 0.6 wt.\% Mg, 61.4 wt.\% Al, 0.1 wt.\% Si, 2.9 wt. \% Mn, 9 wt. \% Fe and 25.4 wt.\% Cu. The elemental composition of the dissolved particle boundary region, as indicated in Figure 15, had an average composition of 1.43 wt.\% Mg, 76.1 wt.\% Al, 1.3 wt. \% Mn, 3.6 wt. \% Fe and 16.7 wt.\% Cu. The increase in $\mathrm{Mg}$ and decrease in $\mathrm{Al}, \mathrm{Mn}, \mathrm{Fe}$ and $\mathrm{Cu}$ indicates that the laser processing began to dissolve the very large $2^{\text {nd }}$ phase particle which resided in the near surface region of the 2024 alloy.
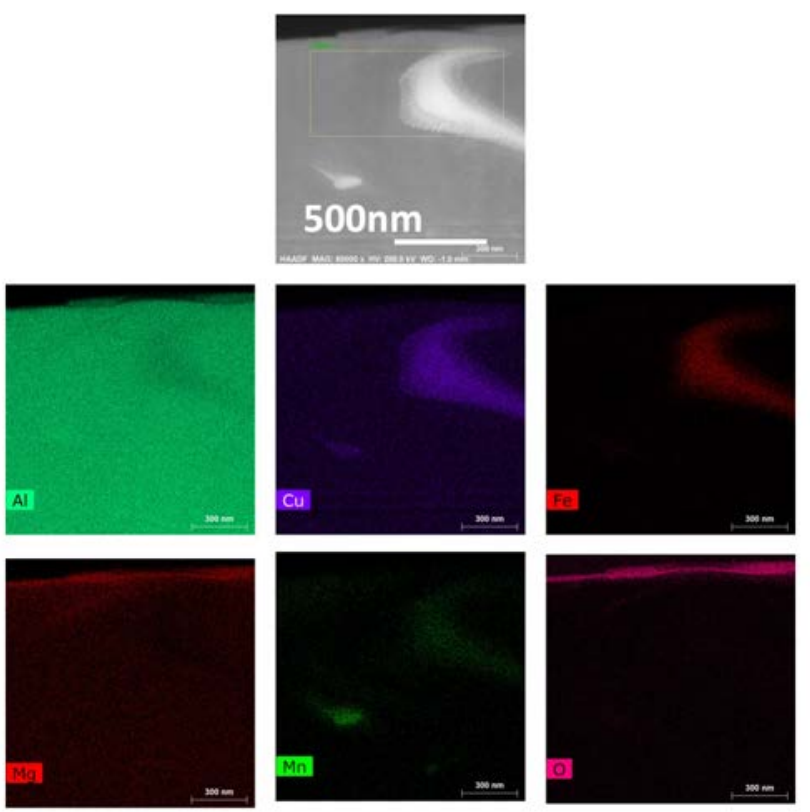

Figure 15. Maps for HAADF STEM image for elements $\mathrm{Al}, \mathrm{Cu}, \mathrm{Fe}, \mathrm{Mg}, \mathrm{Mn}$, and $\mathrm{O}$. Data for the vertical cross-section of the laser-interference processed specimen A1_8s with 8 shots per spot. 


\section{SURFACE CHEMISTRY ANALYSIS}

\subsection{XPS SURFACE ANALYSIS OF AA 2024-T3 SURFACES: AS-RECEIVED, UNPROCESSED}

Data were acquired first on the as received (no cleaning, no laser processing) Al-2024 specimen. Three separate analysis areas were examined (Table 8). First, the XPS measurement was conducted without any $\mathrm{Ar}^{+}$etch (labeled "surf." in Table 8) to reveal the surface contaminants. A wide energy range survey spectrum was first acquired to determine all elements present and then a set of narrow energy range core level spectra were acquired for each identified element. Second, after the initial examination at one analysis area, a very short (labeled "30 sec" in Table 8) Ar-ion etch was performed to remove surface adsorbed contaminants. This short duration etch removes approximately $5 \mathrm{~nm}$ of material at the surface of the specimen based on the calibrated sputter rate of $10 \mathrm{~nm} / \mathrm{min}$ and it was used to reveal the surface of the material. Third, at a second analysis spot, a full depth profile was done using the same ion gun conditions for a total of $\sim 2600 \mathrm{sec}$, approximately $500 \mathrm{~nm}$ depth below the surface, to reveal bulk composition.

Table 8. Overall composition (at.\%) in three areas for the Al-2024 baseline specimen (A0), without any laser processing, in the as-received condition

\begin{tabular}{|l|l|l|l|l|l|l|l|l|l|l|l|l|l|}
\hline Area & XPS & Al & Mg & $\mathbf{C u}$ & $\mathbf{O}$ & $\mathbf{C}$ & $\mathbf{S i}$ & $\mathbf{N a}$ & $\mathbf{C l}$ & $\mathbf{S}$ & $\mathbf{F e}$ & $\mathbf{N}$ & $\mathbf{M n}$ \\
\hline 1 & Surf. & 0.9 & 2.6 & 0.0 & 8.7 & 86.2 & 0.6 & 0.5 & 0.2 & 0.2 & 0.1 & 0.1 & - \\
\hline 2 & Surf. & 1.2 & 3.2 & 0.0 & 9.2 & 84.7 & 0.4 & 0.5 & 0.2 & 0.3 & 0.1 & 0.2 & - \\
\hline 3 & Surf. & 1.2 & 3.1 & 0.0 & 9.4 & 84.6 & 0.5 & 0.4 & 0.2 & 0.3 & 0.2 & 0.1 & - \\
\hline & & & & & & & & & & & & & \\
\hline 1 & $30 \mathrm{~s}$ & 12.8 & 28.0 & 0.2 & 29.7 & 28.0 & - & 0.8 & 0.4 & - & 0.1 & - & 0.1 \\
\hline & & & & & & & & & & & & & \\
\hline 2 & $2600 \mathrm{~s}$ & 87.9 & 1.7 & 3.0 & 1.6 & 5.4 & - & - & - & - & 0.2 & - & 0.3 \\
\hline
\end{tabular}

Surf. = XPS measurement conducted without any $\mathrm{Ar}^{+}$etch

The first three data lines in Table 8, shows overall composition of the as-received surface (without any laser processing). Carbon was the most prevalent element observed, comprising $\sim 85$ atomic $\%$ of the surface with $\mathrm{Mg}, \mathrm{Al}$, and $\mathrm{O}$ making up $\sim 13$ atomic $\%$. The remaining 2 atomic $\%$ was comprised of varying levels of $\mathrm{Si}, \mathrm{Na}, \mathrm{Cl}, \mathrm{S}, \mathrm{N}$ and $\mathrm{Fe}$. Significantly, no $\mathrm{Cu}$ was observed although it is the second 
most abundant element in bulk Al-2024. The fourth line in Table $8(30 \mathrm{sec})$ gives the surface composition data that was acquired after $30 \mathrm{sec}$ of Ar-ion etching. The brief Ar-ion etch removes about two thirds of the surface carbon. Removal of this outer carbon-rich layer increases the signals from Al, $\mathrm{Mg}$, and $\mathrm{O}$. Likewise, small $\mathrm{Cu}$ and $\mathrm{Mn}$ signals are now observed. The increase in $\mathrm{O}$ would indicate that the layer just under the initial C-rich outer layer is an oxide layer on the Al-alloy surface. This will be examined in more detail using the depth profile data.

One important finding to note is that the $\mathrm{Na}$ and $\mathrm{Cl}$ signals both increased slightly after $30 \mathrm{sec}$ of etching (Table 8), indicating that they are more than just a surface adsorbed species. The data at the end of the profile (5200 sec) shows (a) no more $\mathrm{Na}$ or $\mathrm{Cl}$, (b) nearly complete removal of $\mathrm{C}$ and $\mathrm{O}$, and (c) composition that is close to the bulk.

We next will present depth profile data for the as-received baseline material. For the sake of completeness, examples of the $\mathrm{Al} 2 \mathrm{p}, \mathrm{Mg} 2 \mathrm{p}, \mathrm{C} 1 \mathrm{~s}$, and $\mathrm{O} 1 \mathrm{~s}$ spectra collected at specific sputtering cycles of the depth profile are shown in the appendix of (Sabau, Meyer, and Leonard 2020). Clearly the Al 2p shows both metal and oxide components for intermediate depth. The $\mathrm{Mg}$, however, only showed an oxide component until a depth of approximately $250 \mathrm{~nm}$. A depth profile on the baseline material is shown in Figure 16. Figure $16(\mathrm{a}, \mathrm{b})$ shows the $\mathrm{C} 1 \mathrm{~s}, \mathrm{O} 1 \mathrm{~s}, \mathrm{Al} 2 \mathrm{p}$ (peak fit to give both Al-oxide and Al-metal) and the $\mathrm{Cu} 2 \mathrm{p}$ (multiplied by 5). Figure 16(c) shows the $\mathrm{Mn} 2 \mathrm{p}, \mathrm{Na} 1 \mathrm{~s}$, and $\mathrm{Cl} 2 \mathrm{p}$ profiles plotted on a scale from 0 to 1 at. \%. Based on the analysis of the depth profile for the baseline material the following observations were made. Carbon dropped from $\sim 85$ at. $\%$ to 11 at. $\%$ by a depth of $50 \mathrm{~nm}$ and then to $\sim 5$ at. \% by $100 \mathrm{~nm}$ depth. As the carbon signal decreased, the $\mathrm{Mg}$, Al-oxide/Al-metal, and O signals increased. $\mathrm{O}$ and $\mathrm{Mg}$ continued to increase to a depth of $\sim 30 \mathrm{~nm}$ before decreasing with further sputtering, reaching values of 2.8 and 3.2 at. \%, respectively, at $\sim 400 \mathrm{~nm}$ depth. The Al-oxide profile increased to $\sim 11$ at. $\%$ by a depth of $\sim 20 \mathrm{~nm}$ before it began decreasing with continued sputtering reaching a value of $\sim 4$ at. $\%$ by a depth of $400 \mathrm{~nm}$. As the $\mathrm{O}, \mathrm{Mg}$, and Al-oxide signals approached their maximum values between 20 and $30 \mathrm{~nm}$, the Al-metal signal began to increase rapidly, reaching a value of $\sim 85$ at. $\%$ by a depth of $400 \mathrm{~nm}$ and a final value of $\sim 88$ at. $\%$ by the end of the depth profile (depth $\sim 500 \mathrm{~nm}$ ). Likewise, the $\mathrm{Cu}$ signal emerge and grew at this same depth $(\sim 17 \mathrm{~nm})$ and reached a value of 2.3 at. $\%$ at $400 \mathrm{~nm}$ depth and 2.5 at. $\%$ by the end of the depth profile. 


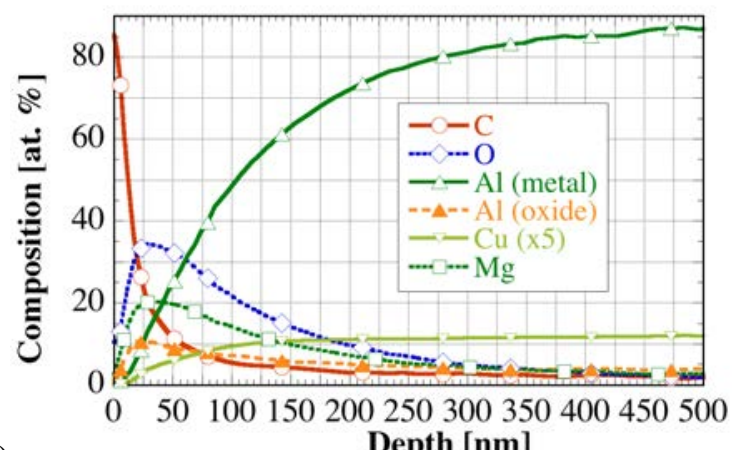

(a)

Depth $[\mathrm{nm}]$

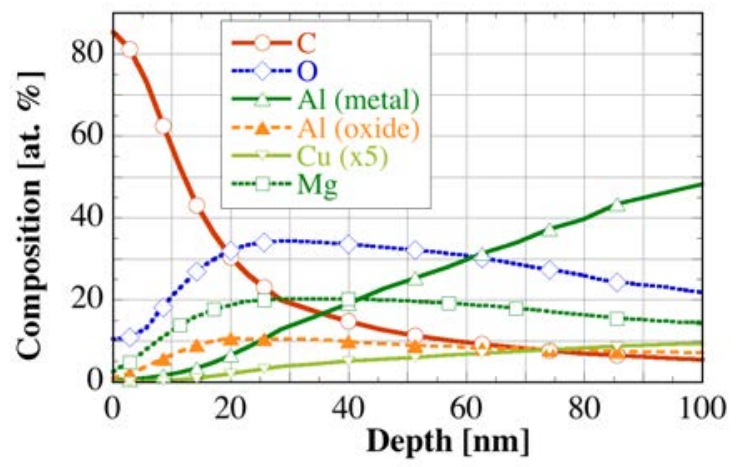

(b)

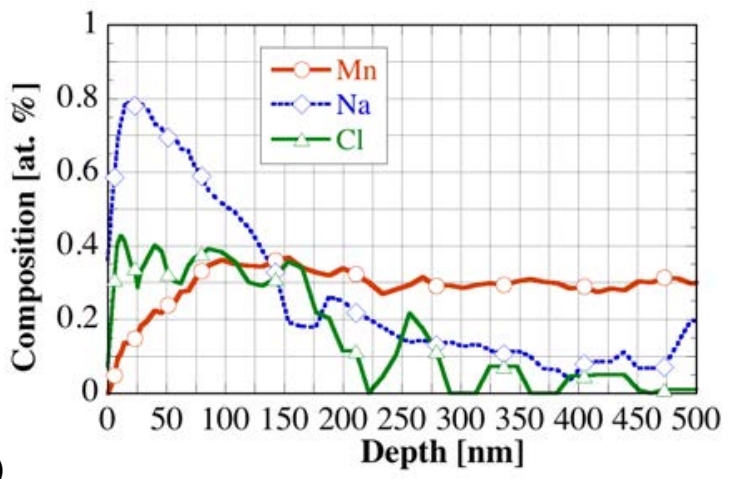

(c)

Depth [nm]

Figure 16. Ar-ion depth profile for AA2024 baseline specimen (A0) (without any laser processing, the asreceived condition: (a, b) $\mathrm{C}$ 1s, $\mathrm{O}$ 1s, Al $2 \mathrm{p}$ (peak fit to give both Al-oxide and Al-metal), $\mathrm{Mg}$, and the $\mathrm{Cu} 2 \mathrm{p}$ signal (multiplied by 5 ) on a scale of 0-100 at. \% and (c) Mn 2p, Na 1s, and Cl 2p profiles.

Like the $\mathrm{Cu}$ signal, $\mathrm{Mn}$ increased from 0 at. \% on the surface to $\sim 0.3$ at. $\%$ by a depth of $100 \mathrm{~nm}$ and then changed very little with further sputtering. The initially weak $\mathrm{Na}$ and $\mathrm{Cl}$ signals increased slightly $(\mathrm{Na}$ from $\sim 0.4$ to $\sim 0.8 ; \mathrm{Cl}$ from $\sim 0.1$ to $\sim 0.4$ ) as the $\mathrm{C}$ was removed from the surface and then were decreased with further sputtering and were essentially gone by a depth of $\sim 200 \mathrm{~nm}$.

The depth profile on the baseline material (A0) yields the following observations. The outermost surface of the as received material has a carbon-rich layer. Underneath this carbon layer is an oxide layer that is comprised of both Al-oxide and Mg-oxide. Because the Al-oxide signal reaches its maximum value a bit sooner, this outer portion oxide is likely a mixed Al-oxide/Mg-oxide near the carbon/oxide interface (5-17 $\mathrm{nm}$ ) and then is a Mg-oxide-rich region from 17-30 $\mathrm{nm}$. As the oxide region is being removed, the base alloy is being revealed as the $\mathrm{Al}, \mathrm{Cu}$, and $\mathrm{Mn}$ signals are observed to increase. An Al-metal signal is observed with initial sputtering (5-10 nm depth) and indicates that the native oxide has thin spots and/or pin-holes. This is supported by the cross-section STEM/EDS data shown in Figure 14 on the oxygen EDS map. There are regions as thick as $\sim 100 \mathrm{~nm}$, but the map shows that thinner regions $(\sim 20 \mathrm{~nm})$ are more 
typical. The data obtained using XPS averages over a much larger area ( 400 micron diameter spot) compared to the cross-section shown in Figure 14 and within that area are likely regions even thinner than $20 \mathrm{~nm}$. At a depth of about $200 \mathrm{~nm}$, the outer layer of (carbon + oxide) materials is mostly gone and the base alloy is observed. At the end of the depth profile, the amounts of $\mathrm{Al}(\sim 88$ at. \%), $\mathrm{Mg}(\sim 2$ at. $\%), \mathrm{Cu}$ $(\sim 3$ at. $\%)$, and $\mathrm{Mn}(\sim 0.3$ at. $\%)$ are near their bulk values of $\mathrm{Al}-96$ at. $\%$; $\mathrm{Mg}-1.5$ at. $\%$; $\mathrm{Cu}-1.9$ at. $\%$; and $\mathrm{Mn}-0.3$ at. $\%$.

For comparing the results of the baseline material with data that will be presented for the laser processed sample, several characteristics of the depth profile were selected. First is the point that the Al-metal signal crosses the Al-oxide signal and is taken as an estimate of the oxide thickness. For the baseline depth profile this occurs at a depth of $\sim 25 \mathrm{~nm}$. Next, we look at the both the maximum values reached by the $\mathrm{Mg}$ and Al-oxide profiles and the depth at which these maximum values are attained. For the baseline material depth profile, the Al-oxide profile reached it maximum composition value of $\sim 11$ at. $\%$ at a depth of $\sim 20 \mathrm{~nm}$, while the $\mathrm{Mg}$ profile reached $\sim 20$ at. $\%$ by $\sim 28 \mathrm{~nm}$ depth.

\subsection{XPS SURFACE ANALYSIS OF AA 2024-T3 SURFACES LASER-INTERFERENCE PROCESSED SPECIMEN A1_2S}

The AA2024 sheet sample A1_2s was laser processed with 2 laser shots per spot using a laser beam angle of $12^{\circ}$. The sample was not cleaned prior to or subsequent to the laser processing. On the as-processed sample, four separate areas were selected for analysis: two areas right at the center of irradiated spots and two areas that were $\sim 1.5 \mathrm{~mm}$ away from the spot center: points 1 and 3 were at the center and points 2 and 4 were near the perimeter. The surface compositions, i.e., without any $\mathrm{Ar}^{+}$etch, are given in Table 9.

Table 9. Surface composition (at.\%), without any Ar+ etch, for the laser processed specimen A1_2s (2shots/spot, laser beam angle of $12^{\circ}$ )

\begin{tabular}{|c|l|l|l|l|l|l|l|l|l|l|}
\hline Area & Location & Al & $\mathbf{M g}$ & $\mathbf{C u}$ & $\mathbf{O}$ & $\mathbf{C}$ & $\mathbf{M n}$ & $\mathbf{S i}$ & $\mathbf{N a}$ & $\mathbf{C l}$ \\
\hline 1 & Spot center & 6.3 & 4.1 & 0.1 & 16.8 & 71.0 & 0.1 & 0.7 & 0.6 & 0.3 \\
\hline 2 & 1.5 mm off center & 7.6 & 4.6 & 0.1 & 17.7 & 68.2 & 0.1 & 0.5 & 0.6 & 0.6 \\
\hline 3 & Spot center & 9.3 & 5.2 & 0.2 & 18.2 & 65.8 & 0.1 & 0.3 & 0.4 & 0.5 \\
\hline 4 & 1.5 mm off center & 7.7 & 5.3 & 0.1 & 17.5 & 67.8 & 0.1 & 0.4 & 0.5 & 0.6 \\
\hline
\end{tabular}


No significant difference was observed between the center and the perimeter and remaining comments pertain to all four areas. Compared to the baseline material, carbon was decreased from $\sim 85$ at. $\%$ to $\sim 68$ at.\%. This modest removal of carbon results in increases of alloy components $\mathrm{Al}$ and $\mathrm{Mg}$. The $\mathrm{O}$ signal also increases, indicating an oxide layer beneath the outer C-layer. Unlike the as received baseline material, a $\mathrm{Cu}$ signal is now observed for the as received laser processed surface. A few, but not all, of the minor components observed on the baseline are still present for the 2-shot laser process, namely $\mathrm{Si}$, $\mathrm{Na}$, and $\mathrm{Cl}$. All values for the elements observed on the as received 2-shot laser processed surface are roughly the same as those observed on the baseline material after $\sim 45 \mathrm{sec}$ of sputtering, or roughly the equivalent of removing $\sim 9 \mathrm{~nm}$ of the outer carbonaceous material (based on examination of the depth profile data of the baseline material). We therefore conclude that the 2-shots into one spot on the alloy surface removes a portion of the carbonaceous outer layer.

A depth profile was next performed on the 2-shot laser processed material at one of the center analysis areas. The depth profile is shown on three plots in Figure 17. Similar to data presented in Figure 16, Figure $17(\mathrm{a}, \mathrm{b}$ ) shows the $\mathrm{C} 1 \mathrm{~s}, \mathrm{O} 1 \mathrm{~s}, \mathrm{Al} 2 \mathrm{p}$ (peak fit to give both Al-oxide and Al-metal) and the $\mathrm{Cu} 2 \mathrm{p}$ (multiplied by 5). Figure 17(c) shows the $\mathrm{Mn} 2 \mathrm{p}, \mathrm{Na} 1 \mathrm{~s}$, and $\mathrm{Cl} 2 \mathrm{p}$ profiles plotted on a scale from 0 to 1 at. \%. Carbon dropped from its initial value of $\sim 50$ at. $\%$ to $\sim 5$ at. $\%$ by a depth of $15 \mathrm{~nm}$ and then to $\sim 2$ at. \% by $100 \mathrm{~nm}$ depth. As the carbon signal decreased, the $\mathrm{Mg}$ and $\mathrm{O}$ signals increased sharply and reached their maximum values at a depth of $\sim 9-10 \mathrm{~nm}$. After that point both $\mathrm{O}$ and $\mathrm{Mg}$ decreased reaching values of $\sim 2.5$ at. $\%$ at $\sim 300 \mathrm{~nm}$ depth. The Al-oxide profile increased to $\sim 23$ at. $\%$ at a depth of $\sim 17 \mathrm{~nm}$ before it began decreasing with continued sputtering reaching a value of 2.8 at. \% by a depth of $300 \mathrm{~nm}$. The Al-metal signal increased linearly with each sputtering increment to $\sim 65$ at. $\%$ by a depth of $100 \mathrm{~nm}$ and then more slowly reaching a value of $\sim 89$ at. $\%$ by the end of the depth profile (depth $\sim 500$ $\mathrm{nm})$. Likewise, the $\mathrm{Cu}$ signal which was already observed on the as received surface grew linearly to about $100 \mathrm{~nm}$ reaching a value of 2.9 at.\%. Above $100 \mathrm{~nm}$ depth, the $\mathrm{Cu}$ profile was nearly flat till the end of sputtering. Similarly, the Mn increased from $\sim 0.1$ at. $\%$ on the surface to $\sim 0.4$ at. $\%$ by a depth of $100 \mathrm{~nm}$ and then changed little with further sputtering. The initially weak $\mathrm{Na}$ and $\mathrm{Cl}$ signals were essentially gone by $\sim 40 \mathrm{~nm}$ depth. 

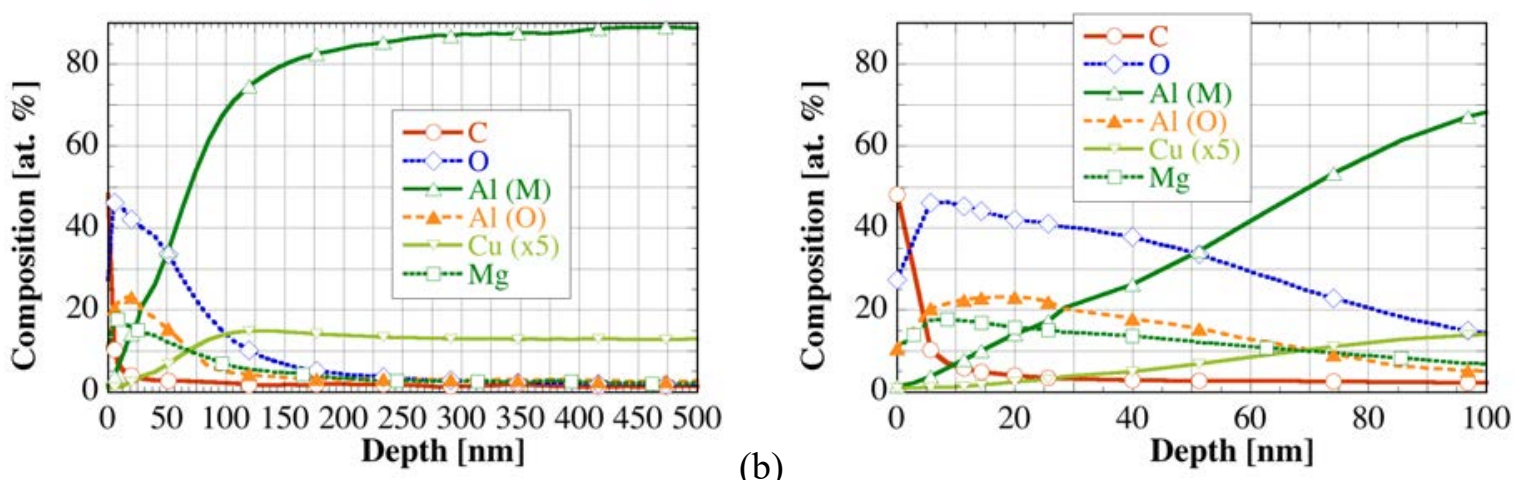

(a)

(b)

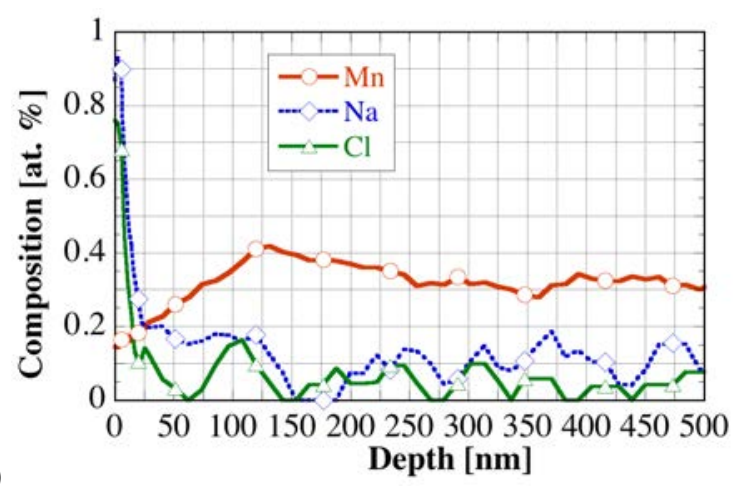

Figure 17. Ar-ion depth profile for A AA2024 laser treated material with 2-shots/spot (A1_2s): (a, b) C 1s, O 1s, Al 2p (peak fit to give both Al-oxide and Al-metal), $\mathrm{Mg}$, and the $\mathrm{Cu} 2 \mathrm{p}$ signal (multiplied by 5 ) and (c) $\mathrm{Mn}$ 2p, Na 1s, and Cl 2p profiles.

The depth profile for the 2-shot laser processed surface shows the following. The outermost surface of the as received material has a thin carbon-rich layer which is mostly removed by the first few sputter increments. Underneath this carbon layer is an oxide layer that is comprised of both Al-oxide and Mgoxide. Differing from the baseline material, the $\mathrm{O}$ and $\mathrm{Mg}$ profile signals peak at a very shallow depth $(\sim 9-10 \mathrm{~nm})$ because the $\mathrm{C}$ outer layer was so thin. The Al-oxide profile signal peaks at a maximum depth of $\sim 20 \mathrm{~nm}$, similar to the baseline material. However, the concentration of the Al-oxide at its maximum is $\sim 23$ at. \% compared to $\sim 11$ at. \% for the baseline sample. Alloy signals belonging to Al-metal, $\mathrm{Cu}$, and Mn were all observed on the as received surface and their values increased with each sputter increment in a linear manner from $0 \mathrm{~nm}$ to $\sim 100 \mathrm{~nm}$. This observation indicates that the 2-shot laser processed surface has thin regions and pin-holes. A cross-section EDS oxygen map (not shown) over a limited region $(\sim 1$ micron) shows much less variation in thickness (35-50 nm thick) compared to the to the baseline material. The much larger areas samples by XPS analysis and depth profiling likely probing thinner areas and pinholes. Above $100 \mathrm{~nm}$ the $\mathrm{Al}-\mathrm{metal}, \mathrm{Cu}$, and $\mathrm{Mn}$ profiles all tend towards plateau values. Compared to the baseline depth profile, the point of crossing of the Al-metal and Al-oxide profiles was nearly the same 
( $28 \mathrm{~nm}$ vs $25 \mathrm{~nm}$ ) but, as mentioned above, the maximum that that the Al-oxide profile reached was greater (23 at. \% vs. 11 at. \%). At the end of the depth profile, the amounts of $\mathrm{Al}(\sim 88$ at. $\%), \operatorname{Mg}(\sim 1.4$ at. \%), $\mathrm{Cu}(\sim 3$ at. \%), and $\mathrm{Mn}(\sim 0.3$ at. \%) are near their bulk values of $\mathrm{Al}-96$ at. \%; $\mathrm{Mg}-1.5$ at. \%; $\mathrm{Cu}-1.9$ at. $\%$; and Mn-0.3 at.\%.

In a previous study on AA5182 alloy (Meyer et al., 2019), it was found that even at 1-shot/spot that the oxide overlayer was thickened compared to the baseline value (75 nm vs. $60 \mathrm{~nm}$ ). We were surprised that in this case it did not do the same. The likely difference was that the baseline material for the AA5128 alloy had only $35-45$ at. \% carbon on the as received surface compared to $>80$ at. $\%$ for the AA2024 alloy. The thicker initial layer of carbon acts to "protect" the native oxide on the as received baseline material and the onset of removal of the oxide is delayed. Because the depth scale on the profile is determined by a calibrated sputter rate and measured sputter time, the thickness of the native oxide on the baseline AA-2024 material appears thicker. Given this observation and the fact that the oxide layer on the 2-shot laser treated surface was slightly thicker ( $28 \mathrm{~nm}$ vs $25 \mathrm{~nm}$ ), we conclude that the oxide layer on the laser treated material was modified by the processing. This claim is supported by the fact that the maximum of the Al-oxide profile for the 2-shot laser treated sample reaches 23 at. \% compared to 11 at. $\%$ for the baseline material.

\subsection{XPS SURFACE ANALYSIS OF AA 2024-T3 SURFACES LASER PROCESSED SPECIMEN A1_8S}

An AA2024 plate sample was laser processed with 8-shots/spot using a laser beam angle of $12^{\circ}$. As before, four separate areas were selected for analysis: two areas right at the center of irradiated spots and two areas that were closer to the perimeter of the circular shot area, $\sim 1.5 \mathrm{~mm}$ away from the spot center: points 1 and 3 were at the center and point 2 and 4 were near the perimeter. The surface compositions, i.e., without any $\mathrm{Ar}^{+}$etch, are given in 
Table 10. 
Table 10. Surface composition (at.\%), without any Ar+ etch, for the laser processed specimen A1_8s (8shots/spot, laser beam angle of $12^{\circ}$ )

\begin{tabular}{|c|l|l|l|l|l|l|l|l|l|l|l|}
\hline Area & Location & Al & Mg & $\mathbf{C u}$ & $\mathbf{O}$ & $\mathbf{C}$ & $\mathbf{M n}$ & $\mathbf{S i}$ & $\mathbf{N a}$ & $\mathbf{C l}$ & $\mathbf{F}$ \\
\hline 1 & Spot center & 18.4 & 3.2 & 0.2 & 32.4 & 43.6 & - & 0.4 & 0.6 & 0.4 & 0.9 \\
\hline 2 & 1.5 mm off center & 20.0 & 5.3 & 0.2 & 36.3 & 35.6 & - & 0.3 & 0.7 & 0.7 & 1.0 \\
\hline 3 & Spot center & 24.1 & 9.3 & 0.2 & 41.6 & 21.7 & - & 0.3 & 0.7 & 0.7 & 1.3 \\
\hline 4 & 1.5 mm off center & 21.8 & 11.4 & 0.3 & 40.7 & 22.7 & - & 0.3 & 0.7 & 0.8 & 1.4 \\
\hline
\end{tabular}

No trend was observed between the center and the perimeter and remaining comments pertain to all 4 areas. Compared to the baseline material (Table 8 for A0), carbon was decreased from $\sim 85$ at. $\%$ to $\sim 30$ at. \%, much more than was observed for the 2-shot laser treated sample. This represents a more significant removal of carbon compared to the 2-shot treatment (Table 9 for A1_s2). Removal of carbon results in increases of alloy components $\mathrm{Al}$ and $\mathrm{Mg}$. Likewise, the $\mathrm{O}$ signal increases indicating that the material below the $\mathrm{C}$-layer is an oxide. As with the 2-shot treatment, a $\mathrm{Cu}$ signal is now observed for the as received laser processed surface where it was not on the baseline as received surface. A few, but not all, of the minor components observed on the baseline are still present for the 2-shot laser process, namely $\mathrm{Si}, \mathrm{Na}, \mathrm{Cl}$ and $\mathrm{F}$. Based on the amount of $\mathrm{C}$ on the as-received 8-shot laser processed surface, the 8shot/spot laser processing was equivalent to the baseline material after $\sim 90 \mathrm{sec}$ of sputtering, or roughly the removing $\sim 18 \mathrm{~nm}$ of outer carbonaceous material. It is clear that the 8 -shots/spot on the alloy surface removes a more significant portion of the carbonaceous outer layer as compared to the 2 -shot processing. After measuring the surface composition of the as-received 8-shot/spot laser processed sample (A1_s8), a depth profile was performed at one of the center analysis areas. The depth profile is shown in Figure 18, in a similar way as those shown in Figure 16 and Figure 17 for the baseline specimen (A0) and 2shot/spot specimen (A1_s2). Carbon dropped from its initial value of $\sim 20$ at. $\%$ to $\sim 3$ at. $\%$ by a depth of $10 \mathrm{~nm}$ and then to $\sim 2$ at. \% by $40 \mathrm{~nm}$ depth. The removal of the outer C-layer increased the $\mathrm{Mg}, \mathrm{Al}-$ oxide, and $\mathrm{O}$ signals sharply, quickly reaching their maximum values by a depth of $\sim 6 \mathrm{~nm}$. Beyond that depth the $\mathrm{O}$ and Al-oxide profiles plateaued at levels of $\sim 40$ at. $\%$ and 30 at $\%$ till a depth of $\sim 50 \mathrm{~nm}$. The $\mathrm{Mg}$ profile decreased continuously after its maximum value of $\sim 12$ at. $\%$ at $6 \mathrm{~nm}$ depth finally reaching a value of $\sim 2$ at. $\%$ by $300 \mathrm{~nm}$ where it remined for the rest of the profile. At $50 \mathrm{~nm}$ depth, the $\mathrm{O}$ and Aloxide profiles decreased steadily thereafter, reaching values of $\sim 2$ and $\sim 5$ at. $\%$ at $300 \mathrm{~nm}$. The Al-metal signal increased linearly with each sputtering increment till about $15 \mathrm{~nm}$ and then plateaued at a value of $\sim 12$ at. \% till about $50 \mathrm{~nm}$ depth. From $50 \mathrm{~nm}$ to $150 \mathrm{~nm}$ the Al-metal signal increased to $\sim 70$ at. \% and finally reached $\sim 85$ at. $\%$ by $300 \mathrm{~nm}$. The $\mathrm{Cu}$ signal which was already observed on the as received surface stayed at $\sim 0.2$ at. \% till a depth of $50 \mathrm{~nm}$ and the grew between 50 and $150 \mathrm{~nm}$ to a value of $\sim 3$ at. $\%$ where it remained for the rest of the profile. The Mn increased from $\sim 0.1$ at. $\%$ on the surface to $\sim 0.3$ 
at. \% by a depth of $150 \mathrm{~nm}$ and then changed little with further sputtering. The initially weak $\mathrm{Na}, \mathrm{Cl}$, and F signals were essentially gone by $\sim 40 \mathrm{~nm}$ depth.
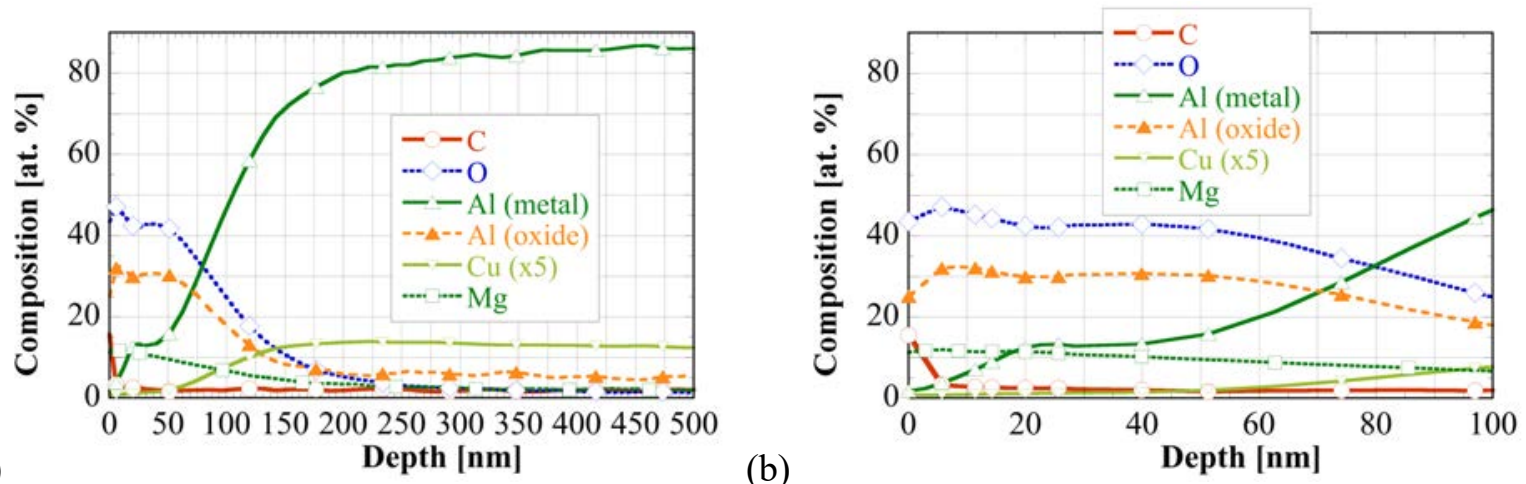

(a)

(b)

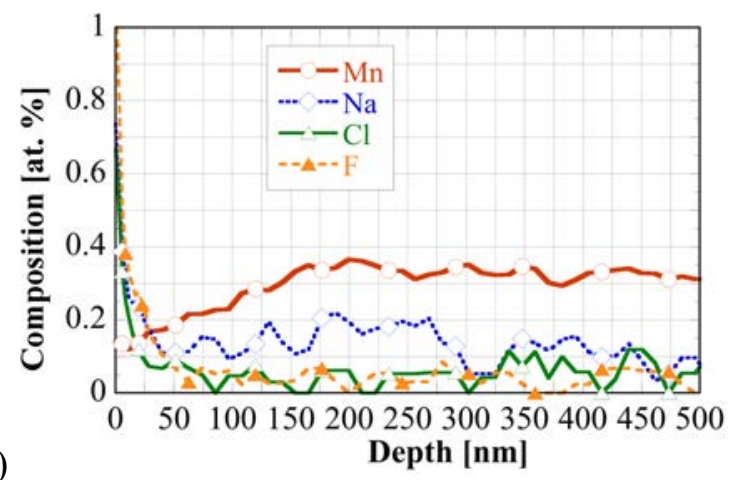

(c)

Depth [nm]

Figure 18. Ar-ion depth profile for A AA2024 laser treated material with 8-shots/spot (A1_8s): (a, b) C 1s, O 1s, Al 2p (peak fit to give both Al-oxide and Al-metal), $\mathrm{Mg}$, and the $\mathrm{Cu} 2 \mathrm{p}$ signal (multiplied by 5) and (c) $\mathrm{Mn}$ $2 p$, Na 1s, and $\mathrm{Cl} 2 \mathrm{p}$ profiles

The depth profile for the 8-shot/spot laser processed surface shows the following. The outermost surface of the as received material has a thin carbon-rich layer which is mostly removed by the first few sputter increments. Underneath this carbon layer is an oxide layer that is comprised of both Al-oxide and Mgoxide. As with the 2-shot/spot sample, the $\mathrm{O}$ and $\mathrm{Mg}$ profile signals peak at a very shallow depth because the $\mathrm{C}$ outer layer was so thin. The $\mathrm{O}$ and $\mathrm{Al}$-oxide profiles reach their maximum values by $\sim 6-9 \mathrm{~nm}$ deep and remain near those values till $\sim 50 \mathrm{~nm}$ deep. The values of the Al-oxide and $\mathrm{O}$ profiles at their maxima are $\sim 30$ at. $\%$ and 40 at. $\%$, respectively. These maxima are higher than both the baseline and 2-shot samples. The Al-metal profile jumps sharply with the removal of the outer C-layer, then stays flat till about a depth of $20 \mathrm{~nm}$ before rising steadily until $\sim 150 \mathrm{~nm}$ where it begins to approach its maximum value of $\sim 88$ at $\%$. The point of crossing of the Al-metal and Al-oxide profiles was at $\sim 75 \mathrm{~nm}$, which is much higher than the values for the 2-shot or baseline values of $28 \mathrm{~nm}$ and $25 \mathrm{~nm}$, respectively. 
Therefore, we conclude that a mixed Al-oxide/Mg-oxide region exists just under the thin $\mathrm{C}$-rich layer and extends to $50-75 \mathrm{~nm}$ deep. Because a clear Al-metal signal is observed in this $50 \mathrm{~nm}$ range, there must be regions that are much thinner than $50 \mathrm{~nm}$. In other words, the $50 \mathrm{~nm}$ Al-oxide/Mg-oxide layer is of nonuniform thickness, consistent with the STEM micrographs. Figure 15 shows an EDS oxygen map that varies in thickness from $\sim 35$ to $75 \mathrm{~nm}$ over a length scale of $\sim 1$ micron. Clearly the larger sampling area of the XPS analysis is observing thinner regions and pin-holes. Alloy signals belonging to $\mathrm{Cu}$ and $\mathrm{Mn}$ were observed on the as received surface and remained low during the first $50 \mathrm{~nm}$ of sputtering before increasing to their plateau values of $\sim 2$ and 0.3 at. \%. At the end of the depth profile, the amounts of Al ( $~ 88$ at. \%), $\mathrm{Mg}(\sim 2.0$ at. \%), $\mathrm{Cu}(\sim 2$ at. \%), and $\mathrm{Mn}(\sim 0.3$ at. \%) are near their bulk values of Al-96 at. \%; $\mathrm{Mg}-1.5$ at. $\%$; $\mathrm{Cu}-1.9$ at. $\%$; and $\mathrm{Mn}-0.3$ at. $\%$.

In Figure 19, the depth profiles of the A1-oxide are shown for the Baseline (A0), 2-Shot (A1_s2) and 8Shot (A1_s8) specimens. From the data shown in Figure 19, the thickness of the Al-oxide can be compared for the Baseline, 2-Shot and 8-Shot specimens. The data clearly shows that the nominal thickness of the $\mathrm{Al}$ oxide layer is larger than that for the baseline specimen and that the oxide thickness increases with the number of shots per spot. The larger value of the Al-oxide concentrations for the laserstructured specimens than that for the baseline specimen at depths less than $50 \mathrm{~nm}$ indicates that there is more oxide material for the laser-structured specimens than that for the baseline surface. The steeper slope in the concentration profiles indicates a more uniform oxide-alloy interface for the laser-structured specimens.

(a)

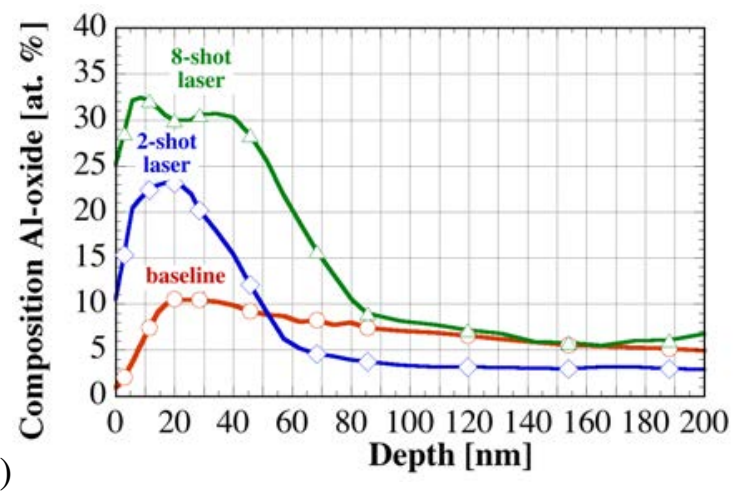

Figure 19. Al-oxide depth profiles (at. \%) for baseline and laser-interference processed specimens A1_2s and A1_8s. 


\subsection{EFFECT OF LASER BEAM ANGLE ON SURFACE COMPOSITION}

Specimens were processed at two other laser beam angles larger than the first beam angle of $12^{\circ}(\mathrm{A} 1)$; namely $36^{\circ}$ (A2) and $72^{\circ}$ (A3). Based on the reduction of the amount of carbon and the increase of A1, $\mathrm{Mg}$, and $\mathrm{Cu}$ on the surface following laser processing, changing the angle of incidence did not have a dramatic effect on the as received surface composition (Table 11) for the 2-shot/spot. For the 8 -shot/spot processing, the angle change from $12^{\circ}$ (A1) to either $36^{\circ}$ (A2) or $72^{\circ}$ (A3) may have decreased the effectiveness of laser removal of surface carbon (Table 11).

Table 11. Surface Composition (at.\%), without any Ar+ etch, for 2 -shot/spot and 8-shot/spot specimens processed with all three beam angle considered

\begin{tabular}{|c|c|c|c|c|c|c|c|c|c|c|}
\hline Specimen & $\begin{array}{c}\text { Beam } \\
\text { Angle [ }^{\mathbf{0}} \text { ] }\end{array}$ & $\mathbf{A l}$ & $\mathbf{M g}$ & $\mathbf{C u}$ & $\mathbf{O}$ & $\mathbf{C}$ & $\mathbf{S i}$ & $\mathbf{N a}$ & $\mathbf{C l}$ & $\mathbf{F}$ \\
\hline A1_2s (Avg.) & 12 & 7.7 & 4.8 & 0.1 & 17.5 & 68.3 & 0.5 & 0.5 & 0.5 & - \\
\hline A2_2s & 36 & 5.1 & 4.3 & 0.1 & 11.7 & 78.5 & 0.0 & 0.2 & 0.2 & - \\
\hline A3_2s & 72 & 5.2 & 5.5 & 0.2 & 25.9 & 61.9 & 0.5 & 0.5 & 0.3 & - \\
\hline A1_8s (Avg.) & 12 & 21.1 & 7.3 & 0.2 & 37.8 & 30.9 & 0.3 & 0.7 & 0.6 & 1.1 \\
\hline A2_8s & 36 & 14.3 & 6.1 & 0.1 & 26.2 & 52.5 & 0.3 & 0.2 & 0.3 & 0.0 \\
\hline A3_8s & 72 & 12.2 & 5.1 & 0.1 & 22.2 & 59.3 & 0.6 & 0.2 & 0.3 & 0.0 \\
\hline
\end{tabular}

Figure 20 compares the Al-oxide depth profiles for the three angles using (a) 2-shots/spot and (b) 8shots/spot. For the 2 shots/spot series, using the point at which the Al-oxide crosses the Al-metal (not shown) as a measure of the oxide thickness, all three process conditions, i.e. angles, give approximately the same thickness of $\sim 28-30 \mathrm{~nm}$. However, the overall amount of Al-oxide, as measured by the maximum in the Al-oxide depth profiles, changes a bit from $\sim 20$ at. $\%$ for $72^{\circ} ; \sim 23$ at. $\%$ for $12^{\circ}$; to $\sim 25$ at. $\%$ for $36^{\circ}$. This implies that an optimum process angle, at least in terms of creating more Al-oxide, exists as one goes through all possible angles from $12^{\circ}$ to $72^{\circ}$, and is likely near $36^{\circ}$. A similar inspection of the 8-shot/spot data shows that all three process angles produces a maximum amount of Al-oxide of $\sim 30$ at. \%. However, the thickness for each process angle, again as measured by the crossing of the Aloxide and Al-metal profiles (not shown), shows differences. For the $36^{\circ}$ angle data, the Al-oxide/Almetal crossing is at $\sim 20 \mathrm{~nm}$, the shallowest, while the $12^{\circ}$ angle data shows a crossing at $\sim 70 \mathrm{~nm}$, the deepest. The $72^{\circ}$ data show an intermediate value of $\sim 50 \mathrm{~nm}$. This indicates that a beam angle exists between $12^{\circ}$ to $72^{\circ}$ that creates a minimum of Al-oxide thickness and that thicker oxides are produced at smaller and larger process angles. These results indicate that the beam angle is an effective process 
variable to control the oxide thickness. However, for a more specific determination of the minimum and maximum oxide thickness from a process map, more beam angles would need to be examined.

(a)

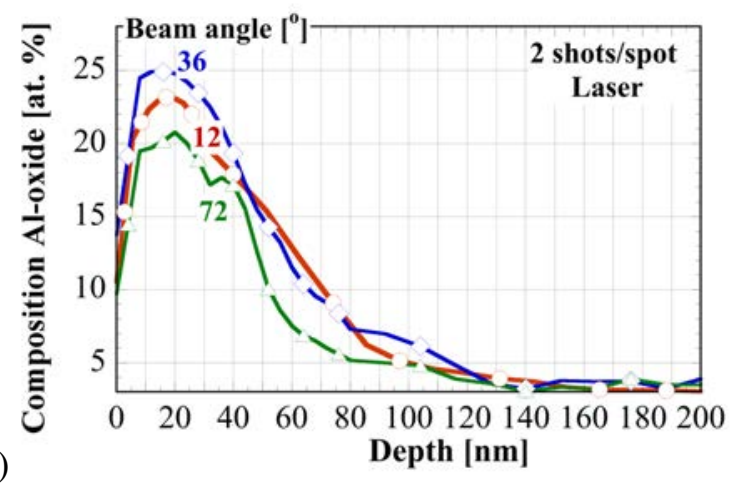

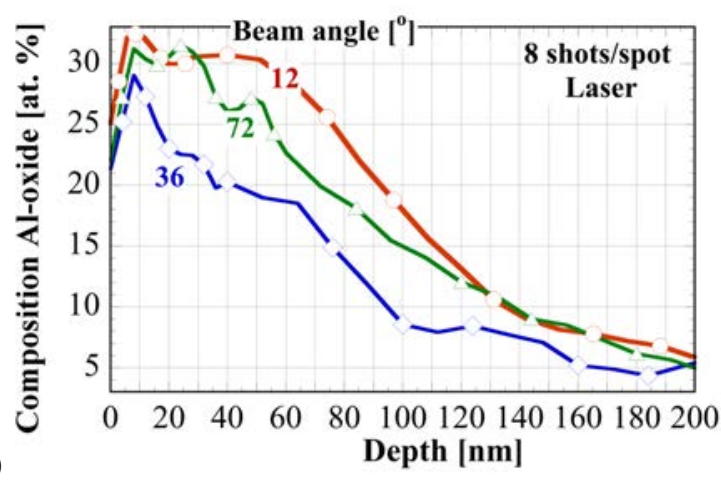

Figure 20. Depth profiles for Al-oxide (at. \%) for laser-interference structured specimens with laser-beam angles of $12^{\circ}, 36^{\circ}$ and $72^{\circ}$ for (a) 2 shots/spot (specimens A1_2s, A3_2s, and A2_2s) and (b) 8 shots/spot (specimens A1_8s, A3_8s, and A2_8s). 


\section{WETTING BEHAVIOR AND SURFACE ENERGIES OF SPOT-BY-SPOT LASER- INTERFERENCE}

Ten laser-interference conditions were used to process specimens for contact angle measurement and subsequent estimate of surface energies (Table 12). In addition, two baseline specimens were considered: (a) chemical conversion coating (CCC) prepared according to MIL-DTL-5541 type I with Cr(VI), having a gold and/or brown color, and (b) sulfuric acid anodization (SAA) having a silver color. As shown Table 12 , three laser beam angles of 12,72 , and $36^{\circ}$ were considered for which the theoretical periodicity would be $1.7,0.3$, and 0.6 micron, respectively. For each beam angle, specimens were laser-interference processed by varying the number of shots-per-spot from 2 to 8 . In total, 40 specimens were prepared. Four specimens per each condition were prepared such that one specimen was used for contact angle measurements for each of the liquids considered. For spot-by-spot laser-interference structuring, the contact angle measurements were conducted at in the center of selected laser spots and on their boundaries.

Table 12. Laser processing conditions for spot-by-spot for contact angle measurements

\begin{tabular}{|c|c|c|c|}
\hline Labels & A1 & A2 & A3 \\
\hline Laser beam angle & $\mathbf{1 2}^{\mathbf{0}}$ & $\mathbf{7 2}^{\mathbf{0}}$ & $\mathbf{3 6}^{\mathbf{0}}$ \\
\hline $\begin{array}{c}\text { Number of shots } \\
\text { per spot }\end{array}$ & & & \\
\hline 2 & - & A2-2 & A3-2 \\
\hline 4 & - & A2-4 & A3-4 \\
\hline 6 & A1-6 & A2-6 & A3-6 \\
\hline 8 & $1-7$ & A2-8 & A3-8 \\
\hline Periodicity $[\boldsymbol{\mu m}]$ & & 0.3 & 0.6 \\
\hline
\end{tabular}

\subsection{EXPERIMENTAL PROCEDURE FOR CONTACT ANGLE MEASUREMENT}

In this section, surface energies after laser-interference structuring (LIS) of as-received specimens, i.e., without employing any polishing, cleaning or any other surface alteration techniques were estimated. This study on contact angle measurement seeks to clarify open questions on the effectiveness of this laser technique at changing the wetting behavior, given that the height of LIS features are less than 2 microns in height. The equipment used consisted of a standard goniometer (Rame-hart, 260-F4) and an image 
processing software DROPimage Advanced version 2.5.02. Table 13 summarizes the surface tension of the four liquids used for contact angle measurement. Volume of the liquids is controlled at $3 \mu \mathrm{L}$ for contact angle measurement. The surface tension of the liquids was characterized using a BP100 bubble pressure tensiometer (Kruss, Hamburg, Germany) to determine the total surface tension. Then, the dispersive and polar components were determined by their contact angle on a polytetrafluoroethylene (PTFE) plate. The detailed procedure was reported in (Davoodabadi et al. 2018).

Table 13. Surface tension of liquids used for contact angle measurements

\begin{tabular}{|c|c|c|c|}
\hline $\begin{array}{c}\text { Total Surface } \\
\text { Tension } \\
{[\mathbf{m N} / \mathbf{m}]}\end{array}$ & $\begin{array}{c}\text { Dispersive } \\
\text { component }[\mathbf{m N} / \mathbf{m}]\end{array}$ & $\begin{array}{c}\text { Polar component } \\
{[\mathbf{m N} / \mathbf{m}]}\end{array}$ & $\begin{array}{c}\text { Total Surface } \\
\text { Tension } \\
{[\mathbf{m N} / \mathbf{m}]}\end{array}$ \\
\hline Diiodomethane & 42.7 & 42.7 & 0.0 \\
\hline Ethylene Glycol & 48.3 & 28.1 & 20.3 \\
\hline 2,2'Thiodethanol & 54.0 & 39.2 & 14.8 \\
\hline De-ionic water & 71.9 & 19.5 & 52.4 \\
\hline
\end{tabular}

Before measurement, the goniometer was properly calibrated, and the tilt of sample holder baseline was adjusted to zero. The inertia effect on the final liquid drop spread on the surface was avoided following the procedure presented below. A drop of liquid with precise controlled volume was formed in the tip of pipette, and then the pipette was slowly moved down until the liquid droplet touched the spot of interest. Then, the pipette was slowly raised to leave the liquid droplet on the surface. Examples of drops for contact angle measurements can be found in Figure 21. Finally, the contact angle was measured on each side of the drop contacting the surface using the instrument software DROPimage Advanced based on a profile method (Figure 22). The average value of the contact angle on the left and right side was used for further surface energy characterization. Five independent tests were conducted on different spots of the same panel (Figure 21). 

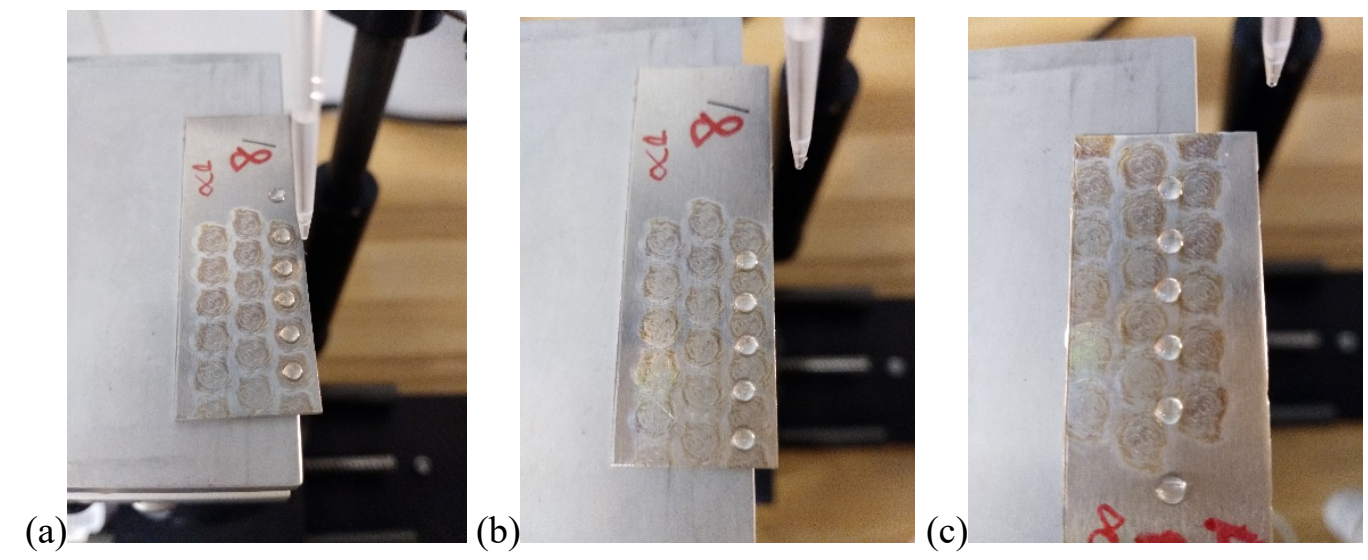

Figure 21. Examples of contact angle measurement on spot-by-spot treated samples (a) laser spot center locations, (b) 2 spot overlap region, and (c) 3 spot overlap region.

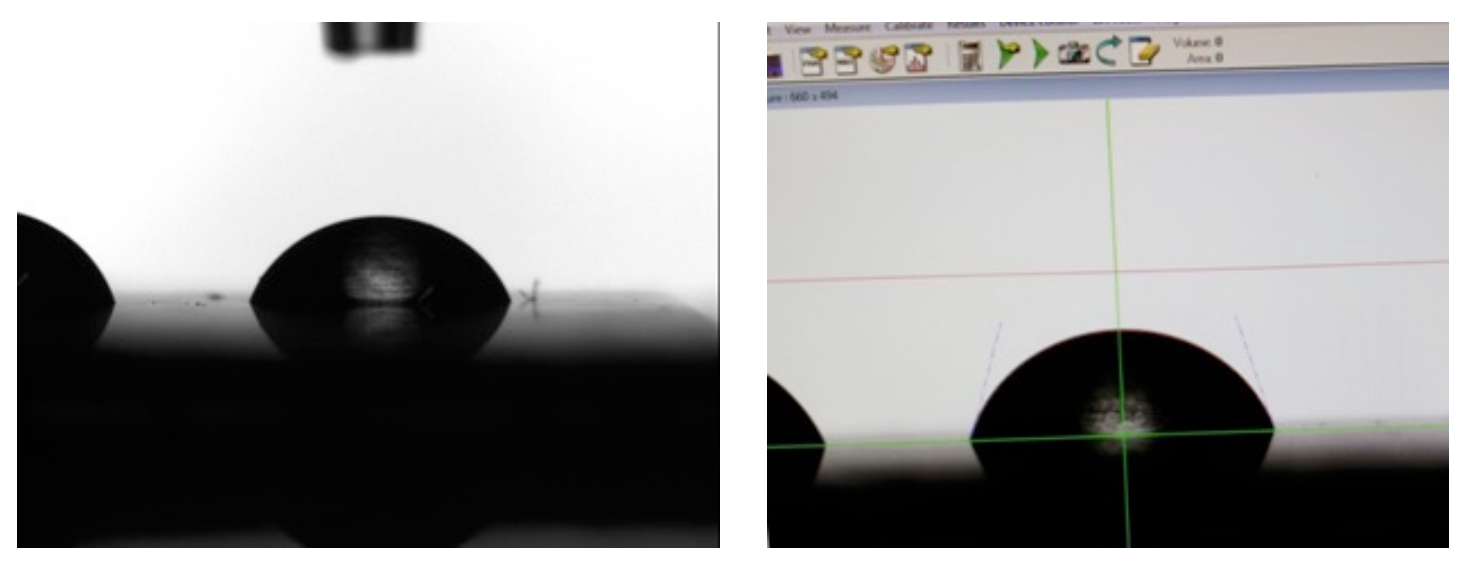

Figure 22. Images of a typical liquid drop (left) and a profile-based contact angle measurement (right).

\subsection{CONTACT ANGLE MEASUREMENTS FOR SPOT-BY-SPOT LASER INTERFERENCE PROCESSED SURFACES}

Contact angle measurements were conducted at three locations for laser-structured spot-by-spot: spotcenter, dual-overlap region, and tri-overlap region in order to assess the variation in the contact angle due to different coverage types. For each fluid, 150 contact angle measurements were conducted for 10 laserstructured surfaces: three locations per each laser condition, and five measurements per each condition investigated. The mean value and standard deviation, $\sigma_{C A}$, for the contact angle was obtained using the 5 measurements conducted at each location. The mean contact angles and their standard deviations are shown Table 15, Table 16, and Table 17 for the baseline conditions, A1, A2, and A3 LIS conditions, respectively. The static contact angle was not available for ethylene glycol on the SAA sample as the ethylene glycol spread out instantly when being added to the sample surface. 
Table 14. Mean contact angle $\left[{ }^{\circ}\right]$ and its standard deviation $\left[{ }^{\circ}\right]$ for the two baseline alloys

\begin{tabular}{|l|c|c|c|c|}
\hline \multicolumn{1}{|c|}{ Liquids } & \multicolumn{2}{c|}{ CCC } & \multicolumn{2}{c|}{ SAA } \\
\hline & Mean $\left[^{\circ}\right]$ & $\sigma_{C A}\left[^{\circ}\right]$ & Mean $\left[^{\circ}\right]$ & $\sigma_{C A}\left[^{\circ}\right]$ \\
\hline Diiodomethane & 54.96 & 0.75 & 9.40 & 0.90 \\
\hline Ethylene Glycol & 53.90 & 1.46 & & \\
\hline 2,2'Thiodethanol & 67.86 & 1.04 & 20.40 & 0.98 \\
\hline \multicolumn{1}{|c|}{ Water } & 90.10 & 2.50 & 27.25 & 12.24 \\
\hline
\end{tabular}

Table 15. Contact angle $\left[^{\circ}\right]$ its standard deviation $\left[^{\circ}\right]$ for A1 samples, LIS with $12^{\circ}$ laser beam angle

\begin{tabular}{|c|c|c|c|c|c|c|}
\hline \multicolumn{7}{|c|}{ Contact angle $\left[{ }^{\circ}\right]$ measured in the laser spot center } \\
\hline Liquids & A1-6 & & A1-8 & & A1-R8 & \\
\hline & Mean $\left[{ }^{\circ}\right]$ & $\sigma_{C A}\left[{ }^{\circ}\right]$ & Mean $\left[^{\circ}\right]$ & $\sigma_{C A}\left[{ }^{0}\right]$ & Mean $\left[{ }^{\circ}\right]$ & $\sigma_{C A}\left[{ }^{\circ}\right]$ \\
\hline Diiodomethane & 48.84 & 2.31 & 43.58 & 1.40 & 45.06 & 1.17 \\
\hline Ethylene Glycol & 73.56 & 0.74 & 72.36 & 0.52 & 72.66 & 1.24 \\
\hline 2,2'Thiodethanol & 68.60 & 1.22 & 67.14 & 0.44 & 66.78 & 0.30 \\
\hline Water & 96.92 & 1.17 & 97.16 & 1.83 & 97.30 & 0.48 \\
\hline \multicolumn{5}{|c|}{ Contact angle $\left[{ }^{\circ}\right]$ measured in the 2 spot overlap region } & \multicolumn{2}{|c|}{$\begin{array}{l}\text { Contact angle }\left[{ }^{\circ}\right] \\
\text { measured in the } 2 \\
\text { spot overlap region }\end{array}$} \\
\hline & Mean $\left[{ }^{\circ}\right]$ & $\sigma_{C A}\left[{ }^{\circ}\right]$ & Mean $\left[{ }^{\circ}\right]$ & $\sigma_{C A}\left[{ }^{\circ}\right]$ & Mean $\left[{ }^{\circ}\right]$ & $\sigma_{C A}\left[{ }^{\circ}\right]$ \\
\hline Diiodomethane & 50.80 & 0.95 & 47.10 & 3.74 & 47.96 & 1.79 \\
\hline Ethylene Glycol & 70.42 & 0.92 & 69.50 & 1.07 & 66.26 & 3.26 \\
\hline 2,2'Thiodethanol & 64.56 & 1.25 & 62.00 & 1.59 & 67.16 & 1.31 \\
\hline Water & 91.32 & 3.65 & 96.10 & 0.46 & 71.20 & 2.40 \\
\hline \multicolumn{5}{|c|}{ Contact angle $\left[{ }^{\circ}\right]$ measured in the 3 spot overlap region } & & \\
\hline & Mean $\left[{ }^{\circ}\right]$ & $\sigma_{C A}\left[^{\circ}\right]$ & Mean $\left[{ }^{\circ}\right]$ & $\sigma_{C A}\left[{ }^{\circ}\right]$ & & \\
\hline Diiodomethane & 58.50 & 4.22 & 47.16 & 0.78 & & \\
\hline Ethylene Glycol & 68.34 & 2.62 & 61.68 & 1.98 & & \\
\hline 2,2'Thiodethanol & 63.64 & 1.71 & 57.54 & 5.14 & & \\
\hline Water & 74.93 & 1.71 & 72.12 & 2.33 & & \\
\hline
\end{tabular}


Table 16. Contact angle $\left[{ }^{\circ}\right]$ its standard deviation $\left[^{\circ}\right]$ for $\mathrm{A} 2$ samples, LIS with $72^{\circ}$ laser beam angle

\begin{tabular}{|c|c|c|c|c|c|c|c|c|}
\hline \multicolumn{9}{|c|}{ Contact angle $\left[{ }^{\circ}\right]$ measured in the laser spot center } \\
\hline Liquids & \multicolumn{2}{|c|}{ A2-2 } & \multicolumn{2}{|c|}{ A2-4 } & \multicolumn{2}{|c|}{ A2-6 } & \multicolumn{2}{|c|}{ A2-8 } \\
\hline & Mean $\left[{ }^{\circ}\right]$ & $\begin{array}{c}\sigma_{C A} \\
{\left[^{\circ}\right]}\end{array}$ & Mean $\left[{ }^{\circ}\right]$ & $\sigma_{C A}\left[^{\circ}\right]$ & Mean $\left[{ }^{\circ}\right]$ & $\sigma_{C A}\left[{ }^{\circ}\right]$ & Mean $\left[{ }^{\circ}\right]$ & $\sigma_{C A}\left[{ }^{\circ}\right]$ \\
\hline Diiodomethane & 47.94 & 2.43 & 49.52 & 2.44 & 46.38 & 3.49 & 42.24 & 1.85 \\
\hline Ethylene Glycol & 73.60 & 1.00 & 73.06 & 1.16 & 71.60 & 0.36 & 71.02 & 1.12 \\
\hline 2,2'Thiodethanol & 69.66 & 1.25 & 65.76 & 1.93 & 62.12 & 0.55 & 65.56 & 1.13 \\
\hline Water & 77.84 & 4.57 & 90.26 & 2.96 & 95.84 & 0.49 & 96.10 & 2.94 \\
\hline \multicolumn{9}{|c|}{ Contact angle $\left[{ }^{\circ}\right]$ measured in the 2 spot overlap region } \\
\hline & Mean $\left[^{\circ}\right]$ & $\begin{array}{c}\sigma_{C A} \\
{\left[^{\circ}\right]}\end{array}$ & Mean $\left[^{\circ}\right]$ & $\sigma_{C A}\left[^{\circ}\right]$ & Mean $\left[{ }^{\circ}\right]$ & $\sigma_{C A}\left[{ }^{\circ}\right]$ & Mean $\left[{ }^{\circ}\right]$ & $\sigma_{C A}\left[^{\circ}\right]$ \\
\hline Diiodomethane & 46.98 & 3.71 & 50.36 & 3.09 & 44.26 & 1.12 & 44.08 & 1.35 \\
\hline Ethylene Glycol & 67.04 & 1.65 & 61.82 & 2.42 & 67.66 & 1.31 & 64.68 & 2.88 \\
\hline 2,2'Thiodethanol & 50.12 & 2.75 & 49.48 & 5.61 & 46.94 & 0.91 & 55.46 & 1.28 \\
\hline Water & 77.84 & 4.57 & 85.08 & 1.37 & 88.56 & 1.42 & 83.54 & 4.75 \\
\hline \multicolumn{9}{|c|}{ Contact angle $\left[{ }^{\circ}\right]$ measured in the 3 spot overlap region } \\
\hline & Mean $\left[^{\circ}\right]$ & $\begin{array}{c}\sigma_{C A} \\
{\left[^{\circ}\right]}\end{array}$ & Mean $\left[^{\circ}\right]$ & $\sigma_{C A}\left[^{\circ}\right]$ & Mean $\left[{ }^{\circ}\right]$ & $\sigma_{C A}\left[{ }^{\circ}\right]$ & Mean $\left[{ }^{\circ}\right]$ & $\sigma_{C A}\left[{ }^{\circ}\right]$ \\
\hline Diiodomethane & 49.58 & 3.79 & 48.74 & 2.64 & 47.62 & 4.22 & 41.12 & 1.37 \\
\hline Ethylene Glycol & 66.98 & 3.58 & 67.22 & 1.91 & 66.92 & 0.82 & 68.42 & 1.00 \\
\hline 2,2'Thiodethanol & 52.90 & 4.07 & 52.70 & 3.99 & 56.44 & 1.72 & 59.20 & 1.95 \\
\hline Water & 82.02 & 3.95 & 79.46 & 2.49 & 89.60 & 1.91 & 89.12 & 2.04 \\
\hline
\end{tabular}


Table 17. Contact angle $\left[^{\circ}\right]$ its standard deviation $\left[^{\circ}\right]$ for A3 samples, LIS with $36^{\circ}$ laser beam angle

\begin{tabular}{|c|c|c|c|c|c|c|c|c|}
\hline \multicolumn{9}{|c|}{ Contact angle $\left[{ }^{\circ}\right]$ measured in the laser spot center } \\
\hline \multirow[t]{2}{*}{ Liquids } & \multicolumn{2}{|c|}{ A3-2 } & \multicolumn{2}{|c|}{ A3-4 } & \multicolumn{2}{|c|}{ A3-6 } & \multicolumn{2}{|c|}{ A3-8 } \\
\hline & $\begin{array}{c}\text { Mean } \\
{\left[^{\circ}\right]}\end{array}$ & $\sigma_{C A}\left[{ }^{\circ}\right]$ & Mean $\left[^{\circ}\right]$ & $\begin{array}{c}\sigma_{C A} \\
{\left[^{\circ}\right]}\end{array}$ & $\begin{array}{c}\text { Mean } \\
{\left[^{\circ}\right]}\end{array}$ & $\begin{array}{c}\sigma_{C A} \\
{\left[{ }^{\circ}\right]}\end{array}$ & Mean $\left[{ }^{\circ}\right]$ & $\sigma_{C A}\left[{ }^{\circ}\right]$ \\
\hline Diiodomethane & 48.58 & 3.17 & 46.78 & 2.27 & 43.88 & 3.42 & 45.55 & 1.40 \\
\hline Ethylene Glycol & 72.84 & 0.44 & 72.74 & 0.60 & 72.04 & 0.45 & 72.02 & 0.90 \\
\hline $\begin{array}{l}\text { 2,2'Thiodethano } \\
1\end{array}$ & 67.30 & 1.24 & 65.92 & 0.55 & 65.24 & 0.87 & 66.22 & 0.37 \\
\hline Water & 95.92 & 1.06 & 94.34 & 0.65 & 95.02 & 1.33 & 95.90 & 1.15 \\
\hline \multicolumn{9}{|c|}{ Contact angle $\left[{ }^{\circ}\right]$ measured in the 2 spot overlap region } \\
\hline & $\begin{array}{c}\text { Mean } \\
{\left[^{\circ}\right]}\end{array}$ & $\sigma_{C A}\left[^{\circ}\right]$ & Mean $\left[{ }^{\circ}\right]$ & $\begin{array}{c}\sigma_{C A} \\
{\left[^{\circ}\right]}\end{array}$ & $\begin{array}{c}\text { Mean } \\
{\left[^{\circ}\right]}\end{array}$ & $\begin{array}{c}\sigma_{C A} \\
{\left[{ }^{\circ}\right]}\end{array}$ & Mean $\left[^{\circ}\right]$ & $\sigma_{C A}\left[^{0}\right]$ \\
\hline Diiodomethane & 42.04 & 1.96 & 47.86 & 3.39 & 43.64 & 3.69 & 49.04 & 2.29 \\
\hline Ethylene Glycol & 60.24 & 1.63 & 65.78 & 2.40 & 62.44 & 1.18 & 64.58 & 1.72 \\
\hline $\begin{array}{l}\text { 2,2'Thiodethano } \\
1\end{array}$ & 46.38 & 2.72 & 55.28 & 2.18 & 44.32 & 2.10 & 52.06 & 1.85 \\
\hline Water & 59.78 & 7.00 & 80.52 & 5.43 & 80.34 & 2.38 & 91.42 & 1.67 \\
\hline \multicolumn{9}{|c|}{ Contact angle $\left[{ }^{\circ}\right]$ measured in the 3 spot overlap region } \\
\hline & $\begin{array}{c}\text { Mean } \\
{\left[^{\circ}\right]}\end{array}$ & $\sigma_{C A}\left[{ }^{\circ}\right]$ & Mean $\left[{ }^{\circ}\right]$ & $\begin{array}{c}\sigma_{C A} \\
{\left[^{\circ}\right]}\end{array}$ & $\begin{array}{c}\text { Mean } \\
{\left[^{\circ}\right]}\end{array}$ & $\begin{array}{c}\sigma_{C A} \\
{\left[^{\circ}\right]}\end{array}$ & Mean $\left[{ }^{\circ}\right]$ & $\sigma_{C A}\left[{ }^{\circ}\right]$ \\
\hline Diiodomethane & 42.98 & 1.53 & 47.82 & 3.21 & 42.02 & 1.02 & 44.06 & 3.84 \\
\hline Ethylene Glycol & 65.36 & 0.99 & 65.12 & 2.79 & 64.48 & 0.85 & 69.20 & 0.80 \\
\hline $\begin{array}{l}\text { 2,2'Thiodethano } \\
1\end{array}$ & 43.96 & 5.03 & 57.98 & 1.94 & 48.92 & 2.42 & 51.46 & 3.49 \\
\hline Water & 84.42 & 8.50 & 86.98 & 2.85 & 84.68 & 2.27 & 93.54 & 0.91 \\
\hline
\end{tabular}

For beam angle of $72^{\circ}$, i.e., specimens A2, the results for contact angle measurements indicate that laserstructured specimens were compatible with chemical conversion coating (Figure 23). Contact angle measurement over the entire surface, i.e., from spot-center to dual-overlap and tri-overlap regions, shows acceptable variation (Figure 24). For specimens A2, the variation in contact angle was estimated for the dual-overlap region and triple-overlap region with respect to that over the spot center. Except for one fluid, contact angles were found to vary within $-5 \%$ to $15 \%$ for the dual-overlap and tri-overlap regions 
(Figure 25). The results for contact angle measurements for LIS-processed specimens with beam angles of $12^{\circ}$ and $36^{\circ}$, i.e., specimens A1 and A3, are shown in Figure 26. As compared with the data shown in Figure 23 LIS-processed specimens with beam angles of $72^{\circ}$, the contact angle data shown in Figure 26 beam angles of $12^{\circ}$ and $36^{\circ}$ indicate that the contact angle exhibits a weak variation with beam angle.

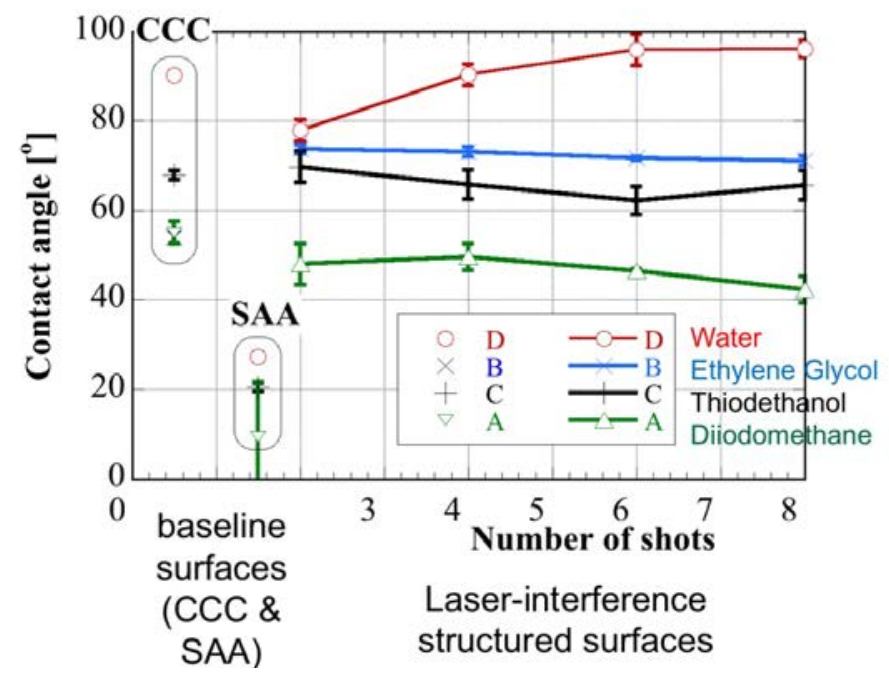

Figure 23. Contact angle for baseline specimens and in the laser-spot center for specimens processed with beam angle of $72^{\circ}$.

(a)

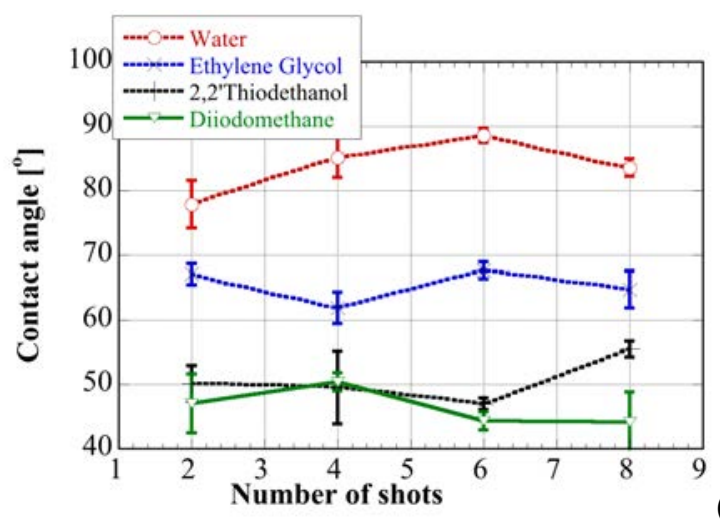

(b)

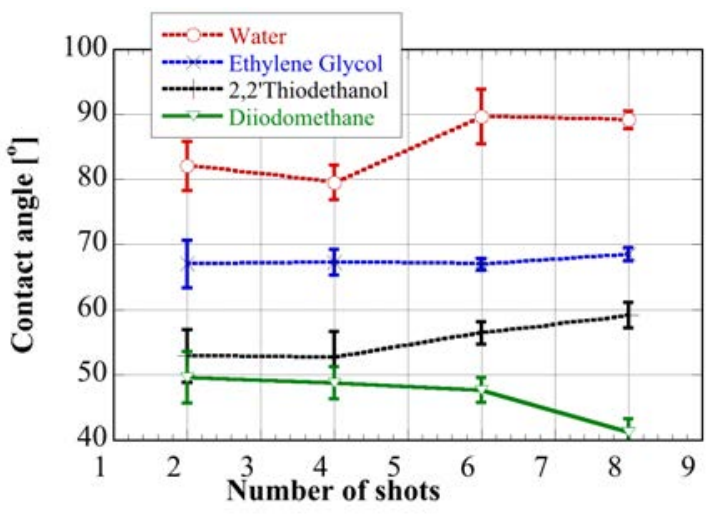

Figure 24. Contact angle for laser-spot center for specimens processed with beam angle of $72^{\circ}$ : (a) dualoverlap and (b) tri-overlap regions. 
(a)

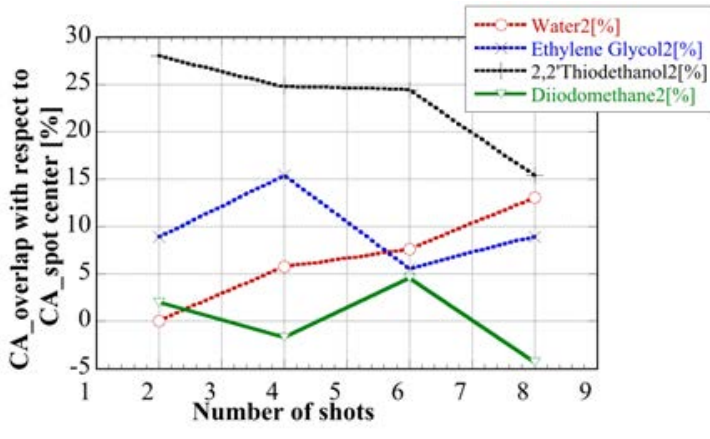

(b)

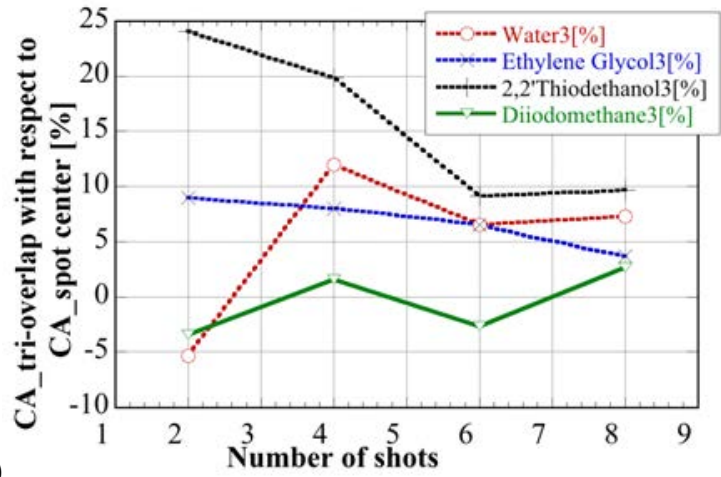

Figure 25. Deviation [\%] of contact angles: (a) dual-overlap regions and (b) tri-overlap regions with respect to the laser spot center (beam angle of $72^{\circ}$ ).

(a)

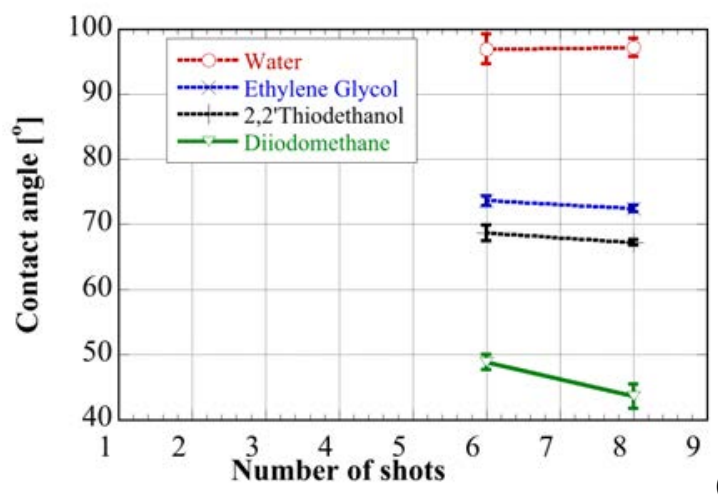

(b)

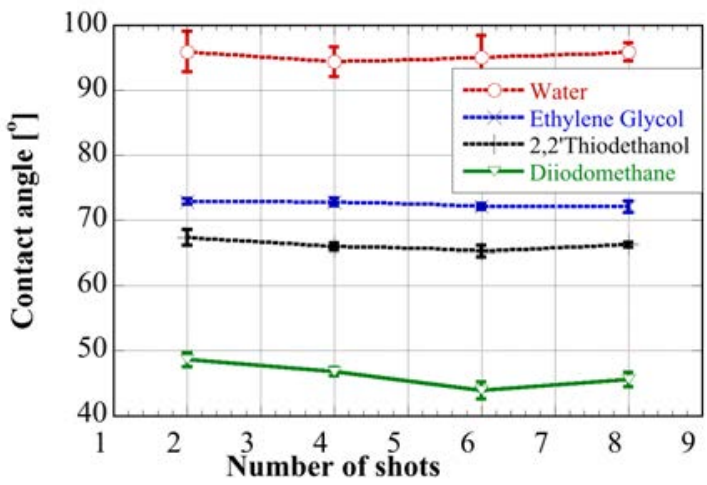

Figure 26. Contact angle in the laser-spot center for specimens processed with beam angles of: (a) $12^{\circ}$ and (b) $36^{\circ}$.

\subsection{RESULTS FOR SURFACE ENERGIES}

The surface energies of the specimens considered were calculated according the Owens-Wendt-RabelKaelble (WORK) method (Owens and Wendt 1969), which is a standard method for calculating the surface energy of a solid from the contact angles with various liquids. The detailed approach was published in (Davoodabadi et al. 2018). As a first approximation, we assumed that the metal surface was ideal, i.e., by not considering the surface porosity and roughness effects in calculating the surface energies. The values of $\mathrm{x}, \mathrm{y}$ components, as shown in Equation 1, are from the probe liquids with known surface tension and measured contact angles. The contact angle results from Table 15, Table 16, and Table 17 for the baseline conditions, A1, A2, and A3 LIS conditions, respectively, were used to obtain the surface energies. The $m$ 
and $c$ are the slope and interception of linear fitted curve of $y$ and $\mathrm{x}$, respectively. The total surface energy of the alloy and relative dispersive and polar components can be calculated according to Equation 2.

$$
\begin{gathered}
\underbrace{\frac{\sigma_{l}(1+\cos \theta)}{2 \sqrt{\sigma_{l}^{l}}}}_{y}=\underbrace{\sqrt{\sigma_{s}^{p}}}_{m} \cdot \underbrace{\sqrt{\frac{\sigma_{l}^{p}}{\sigma_{l}^{d}}}}_{x}+\underbrace{\sqrt{\sigma_{s}^{d}}}_{c} \\
\sigma_{s}=\underbrace{m^{2}}_{\sigma_{s}^{p}}+\underbrace{c^{2}}_{\sigma_{s}^{d}}
\end{gathered}
$$

In this test, the surface energy results are calculated using software SC21 (DataPhysics). Di-ion water was not utilized in surface energy calculation because the correlation coefficient was much lower when including it. The results of surface energy are summarized Table 18 for the baseline conditions and Table 19, Table 20, and 
Table 21 for A1, A2, and A3 LIS conditions, respectively.

Table 18. Calculated surface energies for baseline conditions

\begin{tabular}{|l|l|l|l|l|l|}
\hline OWRK & $\begin{array}{l}\text { Total surface } \\
\text { energy }\end{array}$ & Polar & Dispersive & Polarity & R2 \\
\hline & $\mathrm{mN} / \mathrm{m}$ & $\mathrm{mN} / \mathrm{m}$ & $\mathrm{mN} / \mathrm{m}$ & & \\
\hline SAA & 65.71 & 31.00 & 34.71 & $47.18 \%$ & 0.97 \\
\hline CCC1 & 30.05 & 3.56 & 26.49 & $11.85 \%$ & 0.93 \\
\hline
\end{tabular}

Table 19. Calculated surface energies for A1 samples, LIS with $12^{\circ}$ laser beam angle

\begin{tabular}{|c|l|c|c|c|c|c|}
\hline OWRK & \multicolumn{1}{|c|}{ Location } & Total surface energy & Polar & Dispersive & Polarity & $\mathrm{R}^{2}$ \\
\hline & & $\mathrm{mN} / \mathrm{m}$ & $\mathrm{mN} / \mathrm{m}$ & $\mathrm{mN} / \mathrm{m}$ & & \\
\hline \multirow{4}{*}{ A1-6 } & spot center & 29.91 & 0.32 & 29.58 & $1.06 \%$ & 0.99 \\
\cline { 2 - 7 } & 2 spot overlap & 29.89 & 0.98 & 28.90 & $3.27 \%$ & 0.95 \\
\cline { 2 - 7 } & 3 spot overlap & 27.87 & 2.60 & 25.27 & $9.32 \%$ & 0.95 \\
\hline \multirow{3}{*}{ A1-8 } & spot center & 32.10 & 0.17 & 31.93 & $0.52 \%$ & 0.99 \\
\cline { 2 - 7 } & 2 spot overlap & 31.62 & 0.83 & 30.79 & $2.62 \%$ & 0.94 \\
\cline { 2 - 7 } & 3 spot overlap & 32.91 & 2.38 & 30.53 & $7.23 \%$ & 0.97 \\
\hline
\end{tabular}

Table 20. Calculated surface energies for A2 samples, LIS with $72^{\circ}$ laser beam angle

\begin{tabular}{|c|l|l|c|c|c|c|}
\hline \multirow{2}{*}{ OWRK } & $\begin{array}{l}\text { Total } \\
\text { surface } \\
\text { energy }\end{array}$ & Polar & Dispersive & Polarity & $\mathrm{R}^{2}$ \\
\hline \multirow{3}{*}{ A2-2 } & $\mathrm{mN} / \mathrm{m}$ & $\mathrm{mN} / \mathrm{m}$ & $\mathrm{mN} / \mathrm{m}$ & & \\
\cline { 2 - 7 } & spot center & 30.09 & 0.24 & 29.84 & $0.80 \%$ & 1.00 \\
\cline { 2 - 7 } & 2 spot overlap & 33.76 & 1.74 & 32.02 & $5.15 \%$ & 0.82 \\
\hline \multirow{3}{*}{ A2-4 } & spot center & 32.51 & 1.99 & 30.52 & $6.12 \%$ & 0.84 \\
\cline { 2 - 7 } & 2 spot overlap & 30.10 & 0.50 & 29.60 & $1.66 \%$ & 0.95 \\
\cline { 2 - 7 } & 3 spot overlap & 33.52 & 3.49 & 30.04 & $10.41 \%$ & 0.88 \\
\hline \multirow{3}{*}{ A2-6 } & spot center & 32.78 & 1.83 & 30.95 & $5.58 \%$ & 0.84 \\
\cline { 2 - 7 } & 2 spot overlap & 35.08 & 1.43 & 33.65 & $4.07 \%$ & 0.79 \\
\cline { 2 - 7 } & 3 spot overlap & 32.51 & 1.53 & 30.98 & $4.70 \%$ & 0.89 \\
\hline \multirow{3}{*}{ A2-8 } & spot center & 32.79 & 0.23 & 32.57 & $0.70 \%$ & 0.99 \\
\cline { 2 - 7 } & 2 spot overlap & 33.93 & 1.50 & 34.12 & $4.42 \%$ & 0.91 \\
\cline { 2 - 7 } & 3 spot overlap & 34.15 & 0.56 & 33.59 & $1.64 \%$ & 0.93 \\
\hline
\end{tabular}


Table 21. Calculated surface energies of A3 samples, LIS with $36^{\circ}$ laser beam angle

\begin{tabular}{|c|l|c|c|c|c|c|}
\hline \multirow{2}{*}{ OWRK } & $\begin{array}{c}\text { Total } \\
\text { surface } \\
\text { energy }\end{array}$ & Polar & Dispersive & Polarity & $\mathrm{R}^{2}$ \\
\hline \multirow{3}{*}{ A3-2 } & spot center & $\mathrm{mN} / \mathrm{m}$ & $\mathrm{mN} / \mathrm{m}$ & $\mathrm{mN} / \mathrm{m}$ & & \\
\cline { 2 - 7 } & 2 spot overlap & 30.20 & 0.40 & 29.79 & $1.32 \%$ & 0.98 \\
\cline { 2 - 7 } & 3 spot overlap & 36.44 & 2.54 & 33.90 & $6.97 \%$ & 0.87 \\
\hline \multirow{3}{*}{ A3-4 } & spot center & 36.09 & 1.80 & 34.29 & $4.98 \%$ & 0.79 \\
\cline { 2 - 7 } & 2 spot overlap & 32.73 & 1.83 & 30.90 & $5.59 \%$ & 0.89 \\
\cline { 2 - 7 } & 3 spot overlap & 32.32 & 1.79 & 30.53 & $5.53 \%$ & 0.93 \\
\hline \multirow{3}{*}{ A3-6 } & spot center & 32.27 & 0.24 & 32.03 & $0.74 \%$ & 0.97 \\
\cline { 2 - 7 } & 2 spot overlap & 36.11 & 2.45 & 33.66 & $6.78 \%$ & 0.82 \\
\cline { 2 - 7 } & 3 spot overlap & 35.66 & 1.60 & 34.06 & $4.48 \%$ & 0.84 \\
\hline \multirow{3}{*}{ A3-8 } & spot center & 31.49 & 0.31 & 31.18 & $0.98 \%$ & 0.98 \\
\cline { 2 - 7 } & 2 spot overlap & 33.06 & 2.44 & 30.62 & $7.38 \%$ & 0.87 \\
\cline { 2 - 7 } & 3 spot overlap & 34.37 & 0.99 & 33.39 & $2.88 \%$ & 0.81 \\
\hline
\end{tabular}

For A2 spot by spot treated samples, the total surface energy, and the dispersive component increased with increasing number of shots per spot from 2 to 8 . The polar component increased from 0.24 to $0.52 \mathrm{mN} / \mathrm{m}$, as number of shots per spot increased from 2 to 6 , and decreased to $0.23 \mathrm{mN} / \mathrm{m}$ at 8 shots per spot. The total surface energy of treated spots is also smaller than those in overlap regions, i.e., between 2 and 3 spots. For the 2-spot overlap, the highest surface energy was obtained at 6 shots per spot, while the highest surface energy was obtained in the 3 spot overlap region.

For A3 spot by spot treated samples, the surface energies of in the spot centers, including the dispersive, polar part and total surface energy, are smaller than those at boundaries between 2 and 3 spots. For the spot centers, the total surface energy and the dispersive component reached maximum at 6 shots per spot. The polar component was found to be relatively constant. For the positions outside of the central spots (i.e., regions with the 2 or 3 spot overlap), the total surface energy and dispersive part varied but it seemed independent on the number of shots.

\section{RESULTS FOR COATING ADHESION TESTING OF RASTER SPECIMENS}

Before proceeding to the presentation of the coating adhesion results, the process variables related to the laser processing and coating application are presented. The coating Adhesion Rating (AR) depends on 
the laser fluence, $F_{l}$, laser raster speed, $U$, coating thickness, $C T$, and storage open-time, $t_{o}$. In short notation, $A R$ is a function of four variables $\left(F_{1}, U, C T, t_{o}\right)$. As a proof-of-principle investigation, this study was limited to few combinations of process parameters. In this study, the number of conditions and ranges for the process parameters, which were investigated, include: (a) two values for $F_{l}$ of 1.24 and $1.78 \mathrm{~J} / \mathrm{cm}^{2}$, (b) four raster speeds $U$ of $4,6,8$, and $10 \mathrm{~mm} / \mathrm{s}$; and (c) three open times of 20, 70, and 228 days at which the number of data was larger than five. The values selected for the fluence and raster speeds were based on the study by (Sabau, Greer, et al. 2016) on another Al alloy.

For these conditions, most of the data was available for a coating thickness range of 15 to $23 \mu \mathrm{m}$ (the nominal recommended coating thickness by the primer supplier is $15-30 \mu \mathrm{m}$ with a mean of approximately $23 \mu \mathrm{m}$ ). Moreover, the adhesion results from the panels with the average coating thickness larger than the mean value of $23 \mu \mathrm{m}$ that is specified by the manufacturer are briefly presented in the appendix. The number of conditions were identified in Table 22, where the selection of process parameters for the three analyses conducted in this study are shown.

Table 22. Selection of process parameters for three coating adhesion analyses conducted

\begin{tabular}{|c|c|c|c|c|c|}
\hline $\begin{array}{c}\text { Parameters } \\
\text { Investigated }\end{array}$ & $\begin{array}{c}\boldsymbol{F}_{\boldsymbol{1}} \\
{\left[\mathbf{J} / \mathbf{c m}^{2}\right]}\end{array}$ & $\begin{array}{c}\mathbf{U} \\
{[\mathbf{m m} / \mathbf{s}]}\end{array}$ & $\mathbf{C T}[\boldsymbol{\mu m}]$ & $\boldsymbol{t}_{\boldsymbol{o}}$ [days] & $\begin{array}{c}\text { No. data } \\
\text { sets }\end{array}$ \\
\hline $\begin{array}{c}\text { Fluence and } \\
\text { speed }\end{array}$ & $\begin{array}{c}1.24 \text { and } \\
1.78\end{array}$ & $\begin{array}{c}4,6,8, \text { and } \\
10\end{array}$ & 15 to 23 & All data & $2 \times 4$ \\
\hline Open time & $\begin{array}{c}1.24 \text { and } \\
1.78\end{array}$ & $\begin{array}{c}4 \text { and } 6 \\
\text { combined }\end{array}$ & 15 to 23 & $\begin{array}{c}\text { Six } \\
\text { values }\end{array}$ & 6 \\
\hline $\begin{array}{c}\text { Coating } \\
\text { thickness }\end{array}$ & 1.24 & $\begin{array}{c}4,6,8, \text { and } \\
10\end{array}$ & $\begin{array}{c}15 \text { to } 23, \\
23 \text { to } 30, \\
\text { and } \\
30 \text { to } 44\end{array}$ & All data & 3 \\
\hline
\end{tabular}

\subsection{COATING ADHESION TEST RATINGS}

All primer adhesion results discussed in this section were obtained from the panels with the average thickness of the coating of 15 to $23 \mu \mathrm{m}$, investigating the coating adhesion rating dependence on the other three variables $\left(F_{l}, U, t_{o}\right)$. For most conditions investigated, the AR was measured for at least five specimens per each condition. The AR results can be presented either in a raw format, such as histogram, which counts the number of measurements that have a certain rating, or as an average. The average was applied to the measurement set using several filters. For example, the average adhesion rating over a given coating thickness range can be defined for each laser fluence, $F_{l}$, raster speed, $U$, and open time, $t_{o}$, as: 
$\overline{A R} 1\left(F_{1}, U, t_{o}\right)=\operatorname{AVE}\left[A R\left(F_{1}, U, C T, t_{o}\right)\right]$ for $15 \mu \mathrm{m}<\mathrm{CT}<23 \mu \mathrm{m}$

First, the results for the X-cut adhesion test are presented. Pictures of selected LIS panels after X-cut adhesion tests are shown in Figure 27. The average primer thickness and the adhesion rating, from 0A, 1A, $2 \mathrm{~A}, 3 \mathrm{~A}, 4 \mathrm{~A}$, or $5 \mathrm{~A}$, are indicated at the bottom of each picture. It would have been more appropriate to assign fractional ratings of $4.5 \mathrm{~A}$ and $3.5 \mathrm{~A}$ for the panel shown in Figure 27(b) and Figure 27(c), respectively, based on the fact that its rating was considered to be below the $5 \mathrm{~A}$ but definitely above the $4 \mathrm{~A}$.
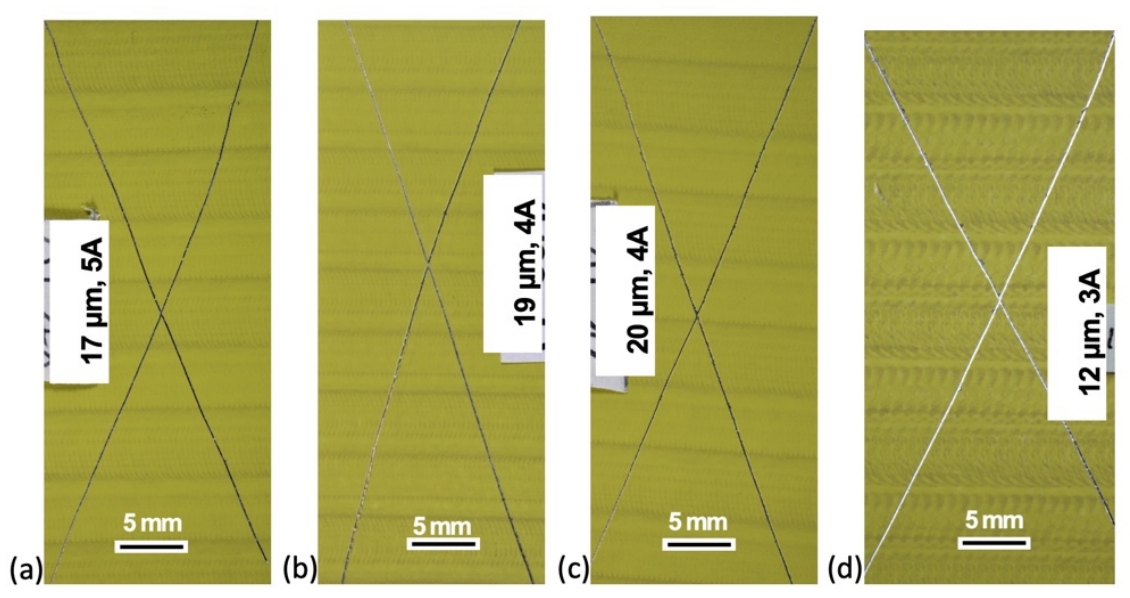

Figure 27. Pictures of $X$-cut tested laser-structured Al panels at rastering speeds of (a-c) $4 \mathrm{~mm} / \mathrm{s}$ and (d) 10 $\mathrm{mm} / \mathrm{s}$ at a laser fluence of $F_{1}=1.238 \mathrm{~J} / \mathrm{cm}^{2}$ per pulse. Coating thickness and $\mathrm{X}$-cut test score according to ASTM D3359, section 7, are indicated for each figure.

Pictures of selected Al control panels after X-cut adhesion test are shown in Figure 28. For the CCC control specimens, the X-cut rating was found to exhibit a large variation, from $5 \mathrm{~A}$ for the specimen shown in Figure 28(a) to 1A for the specimen shown in Figure 28(c). 


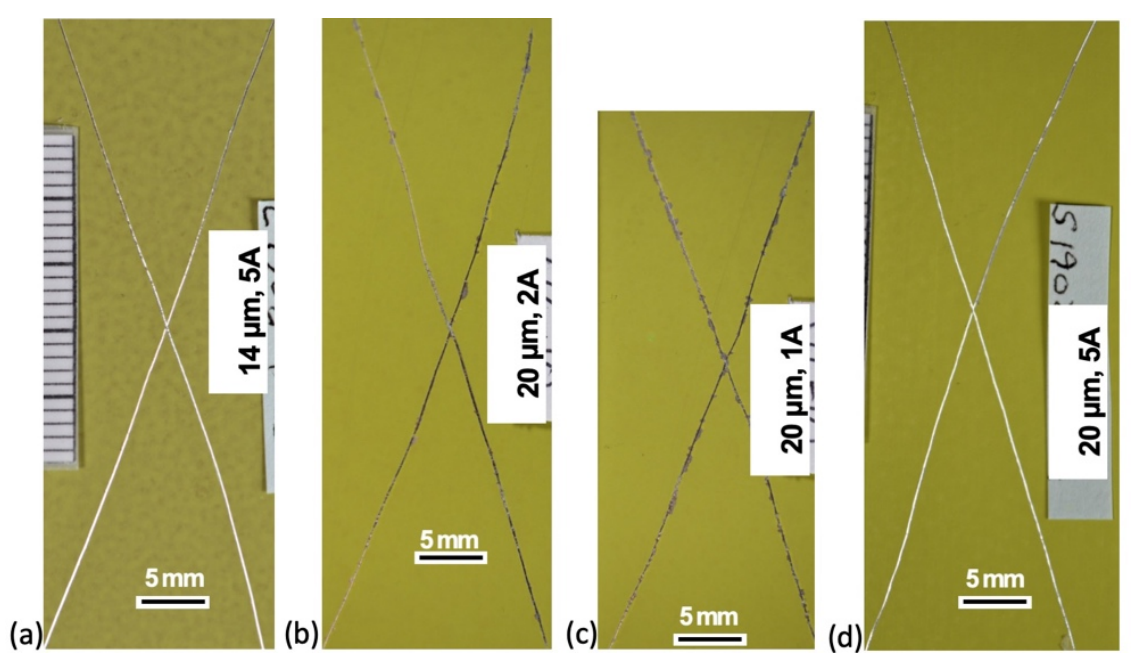

Figure 28. Pictures of X-cut tested Al panels prepared with (a-c) CCC and (d) SAA treatments. Coating thickness and X-cut test score according to ASTM D3359, section 7, are indicated for each figure.

Second, the results for the cross-hatch adhesion tests according to ASTM D3359, Section 12, are presented. Pictures of selected cross-hatch adhesion test are shown in Figure 29 and Figure 30 for six laser-structured specimens and four control specimens, respectively. It would have been more appropriate to assign a fractional rating of 4.5B for the LIS panel shown in Figure Figure 29(d), based on the fact that its rating seemed to be below the 5B but definitely above the 4B. Pictures of selected cross-hatch adhesion test for five control specimens, three specimens prepared with CCC and two specimens prepared by SAA are shown in Figure 30. The coating adhesion of LIS specimens is very good, with some specimens exhibiting the highest coating adhesion rating. Moreover, the cross-hatch test rating of coated LIS specimens outperforms that of the control specimens, which were prepared with CCC. 


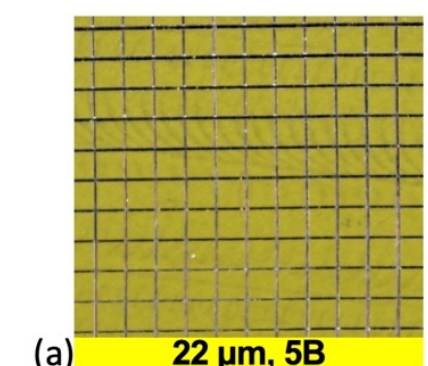

(a)

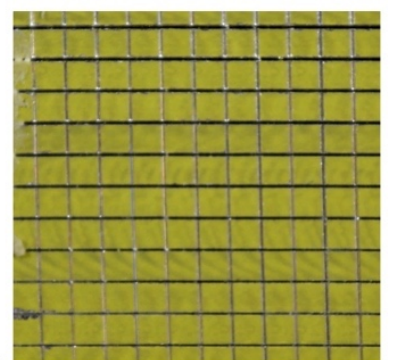

(d)

$19 \mu \mathrm{m}, 4 \mathrm{~B}$

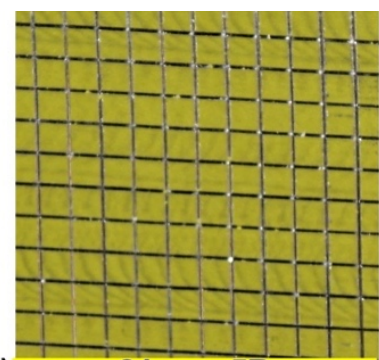

(b)

(e)

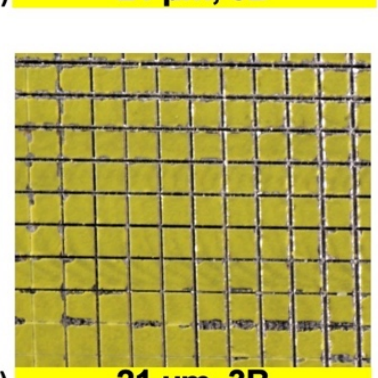

$21 \mu \mathrm{m}, 3 B$

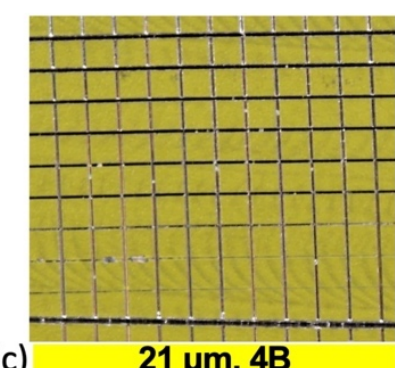

(c)

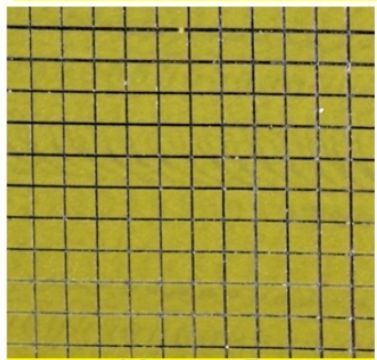

$21 \mu \mathrm{m}, 5 B$

Figure 29. Pictures of cross-hatch tested laser-structured Al panels, test scores (ASTM D3359, section 12), and coating thickness at rastering speed of $(a-c) 6 \mathrm{~mm} \cdot \mathrm{s}^{-1}$ and $(\mathrm{d}-\mathrm{f}) 8 \mathrm{~mm} \cdot \mathrm{s}^{-1}$ at a laser fluence of $F_{1}=1.782$ $\mathrm{J} / \mathrm{cm} 2$ per pulse. Grid size is $1 \mathrm{~mm} \times 1 \mathrm{~mm}$.

(a)

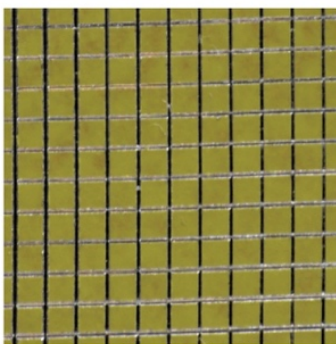

$14 \mu \mathrm{m}, 5 B$

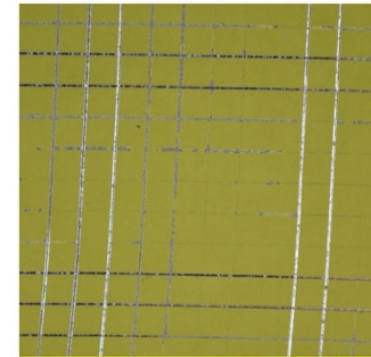

(c)

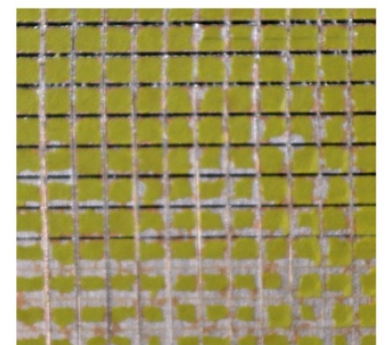

(b)

$18 \mu \mathrm{m}, 2 \mathrm{~B}$

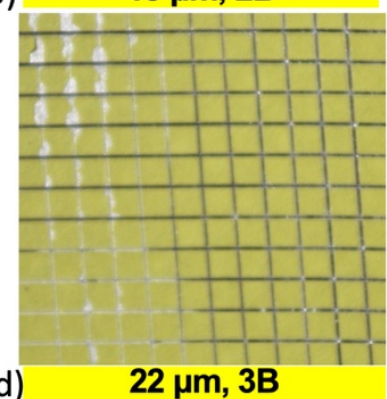

Figure 30. Pictures of cross-hatch tested Al panels test scores (ASTM D3359, section 12), and coating thickness for (a, b) CCC and (c, e) SAA treatments. Grid size is $1 \mathrm{~mm} \times 1 \mathrm{~mm}$.

The results for all of the X-cut and cross-hatch coating adhesion tests, which were conducted on coupons for which the coating thickness was between 15-23 $\mu \mathrm{m}$, are summarized in Figure 31 ( $\mathrm{a}$ and b), respectively, 
for LIS, CCC, and SAA coated panels. In this histogram data, the ratings were combined for all coating sessions, i.e., irrespective of the open-time. The histograms shown in Figure 31 indicate the number of data per each coating adhesion rating, $A R$, for each laser fluence, $F_{l}$, and each raster speed, $\mathrm{U}$. The data shown with empty symbols and solid symbols are for LIS panels with a laser fluence of 1.78 and $1.24 \mathrm{~J} / \mathrm{cm}^{2}$, respectively. As the $\mathrm{X}$-cut test is considered in industry as a less severe test than the cross-hatch test, more specimens were tested for the cross-hatch test than for the X-cut test, once the laser structured specimens would pass this test.

(a)
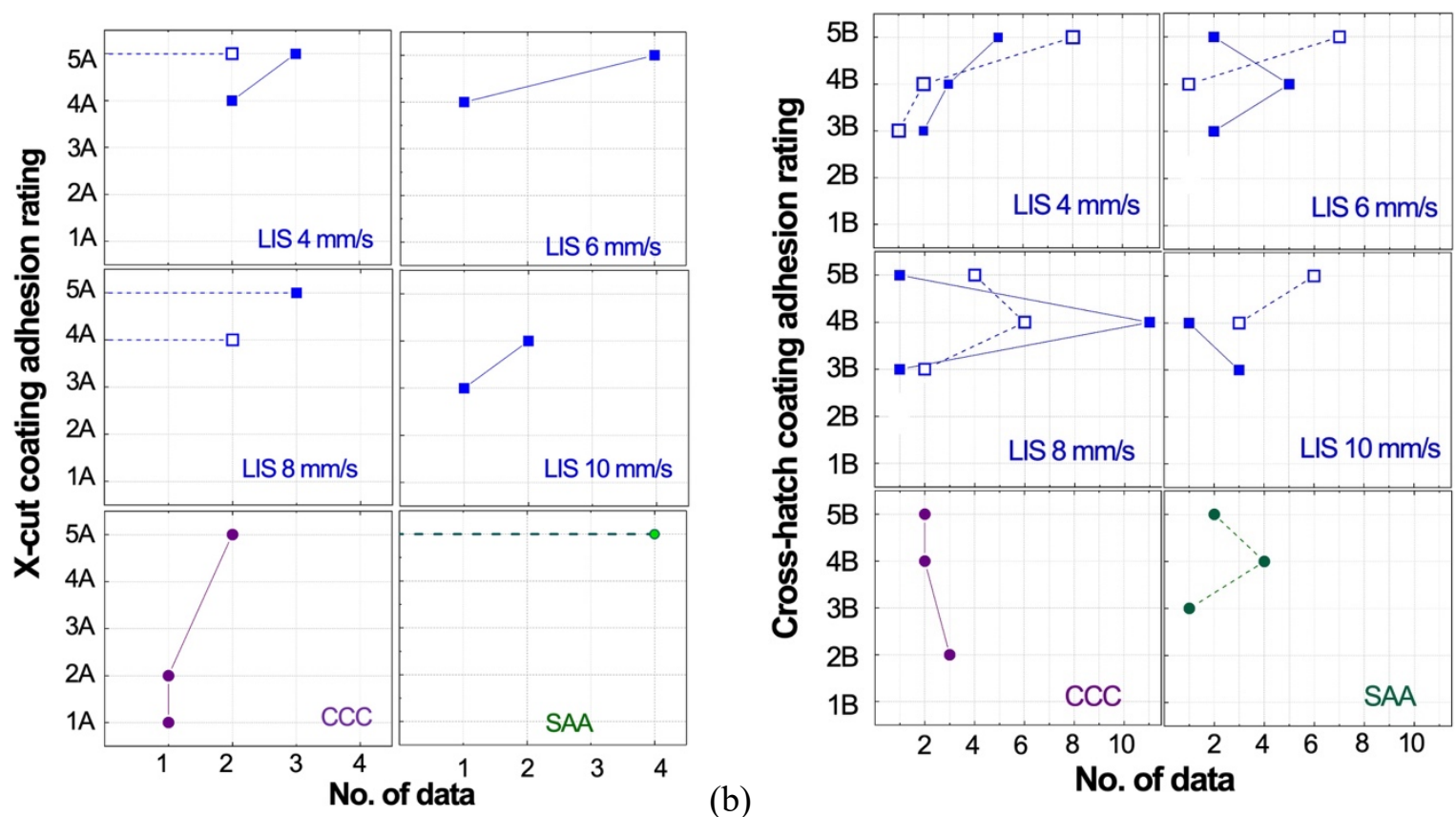

Figure 31. Histograms for coating adhesion rating for LIS, CCC, and SAA coated panels for the: (a) X-cut and (b) cross-hatch tests. The solid symbols and empty symbols indicate data obtained at fluences $F_{1}$ of 1.238 and $1.782 \mathrm{~J} / \mathrm{cm}^{2}$ per pulse, respectively.

For the data shown in Figure 31a, the total number of X-cut tests was 4 and 16 for specimens prepared with laser fluences of $1.78 \mathrm{~J} / \mathrm{cm}^{2}$ and $1.24 \mathrm{~J} / \mathrm{cm}^{2}$, respectively. The calculated average X-cut ratings were 3.3A and 5A for the CCC and SAA controls, respectively. For the laser structured panels with laser fluence of $1.24 \mathrm{~J} / \mathrm{cm}^{2}$, the calculated average X-cut ratings were 4.6A, 4.5A, 5A, and 3.7A for 4, 6, 8, and $10 \mathrm{~mm} / \mathrm{s}$ raster speeds, respectively. For the X-cut test, the SAA panels exhibited the highest adhesion rating while CCC panels showed a spread in the rating indicating quite a variation in the adhesion. Among all of the laser-structured conditions, the LIS panels with $10 \mathrm{~mm} / \mathrm{s}$ raster speed exhibited the lowest rating; however, the average rating for this condition was still above that of the CCC panels. The average X-cut adhesion 
rating for the LIS surface treatment with 4,6 , and $8 \mathrm{~mm} / \mathrm{s}$ was comparable to that for SAA surface treatment panels and higher than that for the CCC panels.

The results of cross-hatch coating tests of primer-coated Al panels are summarized in Figure 31b. For the data shown in Figure 3 lb, the total number of cross-hatch tests was forty-one and thirty-six for laser fluences of 1.78 and $1.24 \mathrm{~J} / \mathrm{cm}^{2}$, respectively. The statistical analysis of the cross-hatch test data shown in Figure $3 \mathrm{lb}$ is now presented. Several statistical Student $\mathrm{t}$-Tests were conducted for the cross-hatch AR. The statistical analysis was performed using the Kaleidagraph software by calculating Satterthwaite's approximate t-Test; a method in the Behrens-Welch family for unpaired data with unequal variance as indicated by (Armitage, Berry, and Matthews 2008). The following variables were computed for each test of unpaired data $(x i ; i=1, N$ and $y j ; j=1, M)$ with unequal variance student $t$-Test, as given by (Armitage, Berry, and Matthews 2008): mean values $(\bar{X}$ and $\bar{Y})$, mean difference $(\bar{X}-\bar{Y})$, standard deviations $\left(\sigma_{x}\right.$ and $\left.\sigma_{y}\right)$, t-Value $t=(\bar{X}-\bar{Y}) / s_{\bar{\Delta}}$ with $s_{\bar{\Delta}}=\sqrt{\sigma_{x}{ }^{2} / N+\sigma_{y}{ }^{2} / M}$, degree of freedom (DOF) as given by the Welch-Satterthwaite equation, and the two-tail (or two-sided) P-value. This P-value, or calculated probability, is the probability of finding the observed value "equal to or more extreme than what was actually observed." In our case, we used the Student t-Tests to determine if there is a statistically significant difference between the two means compared. If this P-value would be below a certain level (e.g., 0.05 or 0.01 ), the conclusion would be that there would be a difference between the two group means, a difference which would be statistically significant at the $95 \%$ or $99 \%$ confidence level, respectively.

The first Student t-Test was used to statistically compare the differences in AR between LIS $\left(1.24 \mathrm{~J} / \mathrm{cm}^{2}\right)$ and LIS $\left(1.78 \mathrm{~J} / \mathrm{cm}^{2}\right)$ at each laser speed. As shown in Table 23, the t-Tests results indicate with $99 \%$ confidence level that each of the following datasets means are statistically different: (a) AR( $1.24 \mathrm{~J} / \mathrm{cm}^{2}, 6$ $\mathrm{mm} / \mathrm{s})$ and $\operatorname{AR}\left(1.78 \mathrm{~J} / \mathrm{cm}^{2}, 6 \mathrm{~mm} / \mathrm{s}\right),(\mathrm{b}) \operatorname{AR}\left(1.24 \mathrm{~J} / \mathrm{cm}^{2}, 10 \mathrm{~mm} / \mathrm{s}\right)$ and $\operatorname{AR}\left(1.78 \mathrm{~J} / \mathrm{cm}^{2}, 10 \mathrm{~mm} / \mathrm{s}\right)$, and (c) $\operatorname{AR}\left(1.24 \mathrm{~J} / \mathrm{cm}^{2}\right.$, all speeds) and $\operatorname{AR}\left(1.78 \mathrm{~J} / \mathrm{cm}^{2}\right.$, all speeds). Here the "all speeds" was used to indicate lumped data for all the speeds. The means for data sets with LIS with 6 and $10 \mathrm{~mm} / \mathrm{s}$ are not statistically different. The second Student t-Test was used to statistically compare the differences in AR data between SAA and LIS $\left(1.78 \mathrm{~J} / \mathrm{cm}^{2}\right)$. As shown in Table 24, the t-Tests results indicate that for all of the individual laser speeds (except $6 \mathrm{~mm} / \mathrm{s}$ ) the AR means for LIS $1.78 \mathrm{~J} / \mathrm{cm}^{2}$ are not statistically different than those for the AR with SAA. The mean values for $6 \mathrm{~mm} / \mathrm{s}$ LIS at $1.78 \mathrm{~J} / \mathrm{cm}^{2}$ are statistically different than the mean for SAA as the mean for this LIS conditions is significantly higher than that for the SAA. 
Table 23. Statistical results from Student t-Tests for individual pairs (LIS-LIS) of cross-hatch ratings at fluences of $1.78 \mathrm{~J} / \mathrm{cm} 2$ and $1.24 \mathrm{~J} / \mathrm{cm} 2$ at each rastering speed

\begin{tabular}{|c|c|c|c|c|c|}
\hline $\begin{array}{c}\text { Rastering } \\
\text { Speed }[\mathrm{mm} / \mathrm{s}]\end{array}$ & $\begin{array}{c}\text { Mean } \\
\text { difference }\end{array}$ & DOF & t Value & P-value & $\begin{array}{c}\text { *Statistically } \\
\text { different }\end{array}$ \\
\hline 4 & 0.34 & 17 & 1.02 & 0.322 & - \\
\hline 6 & 0.88 & 12 & 3.28 & 0.00655 & $\mathrm{Y}$ \\
\hline 8 & 0.17 & 17 & 0.706 & 0.49 & $\mathrm{Y}$ \\
\hline 10 & 1.42 & 5 & 4.72 & 0.00354 & $\mathrm{Y}$ \\
\hline All & 0.49 & 74 & 3.01 & 0.00356 & \\
\hline
\end{tabular}

*statistically significant at the $99 \%$ confidence level, i.e., with P-value $<0.01$.

Table 24. Statistical results from Student t-Tests for individual pairs (SAA-LIS) of cross-hatch ratings. LIS data was at fluences of $1.78 \mathrm{~J} / \mathrm{cm} 2$

\begin{tabular}{|c|c|c|c|c|c|}
\hline $\begin{array}{c}\text { Rastering } \\
\text { Speed }[\mathrm{mm} / \mathrm{s}]\end{array}$ & $\begin{array}{c}\text { Mean } \\
\text { difference }\end{array}$ & DOF & t Value & P-value & $\begin{array}{c}\text { Statistically } \\
\text { different }\end{array}$ \\
\hline 4 & 0.42 & 12 & 1.27 & 0.2283 & $\mathrm{~N}$ \\
\hline 6 & 0.66 & 8 & 2.26 & 0.0513 & $* \mathrm{Y}$ \\
\hline 8 & -0.048 & 12 & -0.142 & 0.8894 & $\mathrm{~N}$ \\
\hline 10 & 0.45 & 10 & 1.45 & 0.1767 & $\mathrm{~N}$ \\
\hline All & 0.27 & 8 & 0.948 & 0.3694 & $\mathrm{~N}$ \\
\hline
\end{tabular}

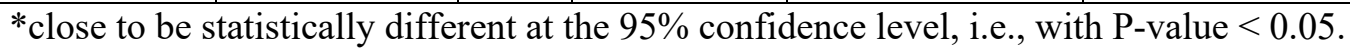

\subsection{DISCUSSION OF COATING ADHESION TESTING RESULTS}

Having established based on the mean values that LIS $\left(1.24 \mathrm{~J} / \mathrm{cm}^{2}\right)$ and $\operatorname{LIS}\left(1.78 \mathrm{~J} / \mathrm{cm}^{2}\right)$ are mainly statistically different and that LIS $\left(1.78 \mathrm{~J} / \mathrm{cm}^{2}\right)$ and SAA (the highest AR for the control specimens) are statistically identical, an analysis of the AR data is made in order to study possible correlations for the coating adhesion performance to the laser processing space. Coating adhesion rating data can be also analyzed by averaging the data for each of the variables considered. The adhesion results from both X-cut and cross-hatch tests are summarized for the average and minimum adhesion ratings, AR, in Table 25. Due to the limited number of data for the X-cut test, the average shown was the proper average for the two laser fluences used instead of showing the averages per each fluence. For the cross-hatch test, both averages at the two laser fluences are shown in Table 25. The standard deviation is also shown for the cross-hatch 
ratings for the specimens prepared with the two laser fluences of 1.24 and $1.78 \mathrm{~J} / \mathrm{cm}^{2}$. Using the $2 \sigma$ as a 95\% confidence level interval, for an assumed student t-distribution of the ratings, the Lower Confidence Limit (LCL) of the mean was calculated and it is shown in Table 25. The LCL can be used as a design basis value for the coating adhesion which represents the minimum value above which $95 \%$ of the coating adhesion ratings would be expected, i.e., minimum property values that would be reached with $95 \%$ confidence. For all the speeds, but the $8 \mathrm{~mm} / \mathrm{s}$, the LCL is higher for LIS at $1.78 \mathrm{~J} / \mathrm{cm}^{2}$ fluence than those at $1.24 \mathrm{~J} / \mathrm{cm}^{2}$ fluence. At $1.78 \mathrm{~J} / \mathrm{cm}^{2}$ fluence, for all of the speeds, but the $8 \mathrm{~mm} / \mathrm{s}$, the LCL is larger than 3 , value which is close to that for the LCL of 2.8 for SAA. At $1.24 \mathrm{~J} / \mathrm{cm}^{2}$ fluence, the LCL is higher than 3 only for the $8 \mathrm{~mm} / \mathrm{s}$. Thus, more laser speeds were found to yield a higher LCL than the SAA at the 1.78 $\mathrm{J} / \mathrm{cm}^{2}$ fluence than at the $1.24 \mathrm{~J} / \mathrm{cm}^{2}$ fluence. A wider laser processing space was found at the $1.78 \mathrm{~J} / \mathrm{cm}^{2}$ fluence than at the $1.24 \mathrm{~J} / \mathrm{cm}^{2}$ fluence.

Based on the X-cut and cross-hatch ratings, surfaces prepared with LIS, at rastering speeds of 4, 6, and 8 $\mathrm{mm} / \mathrm{s}$, and anodizing treatment provided similar adhesion performance, which was higher than that of the $\mathrm{CCC} \mathrm{Al}$ surfaces. Concerning the rastering speed effect on cross-hatch ratings, the following considerations can be made based on the data shown in Table 25:

- The average rating for a laser fluence of $1.24 \mathrm{~J} / \mathrm{cm}^{2}$ is highest at the lowest rastering speed $(4 \mathrm{~mm} / \mathrm{s})$, however, the ratings at the 4 and $6 \mathrm{~mm} / \mathrm{s}$ are very close to each other,

- The average rating for the laser fluence of $1.24 \mathrm{~J} / \mathrm{cm}^{2}$ does not vary significantly as the raster speed is increased to 6 and $8 \mathrm{~mm} / \mathrm{s}$,

- The average rating for the laser fluence of $1.24 \mathrm{~J} / \mathrm{cm}^{2}$ is lowest at the highest rastering speed (10 $\mathrm{mm} / \mathrm{s})$,

- The average rating for the laser fluence of $1.78 \mathrm{~J} / \mathrm{cm}^{2}$ is highest at the rastering speed of $6 \mathrm{~mm} / \mathrm{s}$; however, the ratings at the 4 and $6 \mathrm{~mm} / \mathrm{s}$ are very close to each other,

- With the exception of the $8 \mathrm{~mm} / \mathrm{s}$ series of specimens, the average rating for the laser fluence of $1.78 \mathrm{~J} / \mathrm{cm}^{2}$ does not vary significantly for the other rastering speeds, and it is higher than those for laser fluence of $1.24 \mathrm{~J} / \mathrm{cm}^{2}$,

- The average rating for the laser fluence of $1.78 \mathrm{~J} / \mathrm{cm}^{2}$ is the lowest at the rastering speed of $8 \mathrm{~mm} / \mathrm{s}$,

- Overall, the lower confidence limit for the ratings at a laser fluence of $1.78 \mathrm{~J} / \mathrm{cm}^{2}$ were higher than those ratings at laser fluence of $1.24 \mathrm{~J} / \mathrm{cm}^{2}$,

- The highest ratings, and with the smallest standard deviation, were found for the laser fluence of $1.78 \mathrm{~J} / \mathrm{cm}^{2}$ and lower rastering speeds $(4 \mathrm{~mm} / \mathrm{s}$ and $6 \mathrm{~mm} / \mathrm{s})$. 
Thus, based on the above analysis, the rastering with either $4 \mathrm{~mm} / \mathrm{s}$ or $6 \mathrm{~mm} / \mathrm{s}$ with a laser beam setup at a laser fluence of $1.78 \mathrm{~J} / \mathrm{cm}^{2}$ is recommended to attain acceptable coating adhesion, even higher than that with the anodizing surface treatment.

Table 25. Average, minimum, and lower confidence limit adhesion ratings from $X$-cut and cross-hatch tests for LIS, CCC, and SAA panels coated with CA7233 primer

\begin{tabular}{|c|c|c|c|c|c|c|c|c|c|}
\hline \multirow{2}{*}{$\begin{array}{c}\text { Al surface } \\
\text { treatment }\end{array}$} & $\begin{array}{c}\text { Rastering } \\
\text { Speed } \\
{[\mathrm{mm} / \mathrm{s}]}\end{array}$ & $\begin{array}{c}\text { X-cut rating } \\
\text { (both } \\
\text { fluences) }\end{array}$ & \multicolumn{3}{|c|}{$\begin{array}{c}\text { Cross-hatch rating } \\
\left(\mathrm{F}_{1}=1.24 \mathrm{~J} / \mathrm{cm}^{2}\right)\end{array}$} & \multicolumn{3}{|c|}{$\begin{array}{c}\text { Cross-hatch rating } \\
\left(\mathrm{F}_{1}=1.78 \mathrm{~J} / \mathrm{cm}^{2}\right)\end{array}$} \\
\cline { 3 - 11 } & Avg. & Min. & Avg. & LCL & Std-dev. & Avg. & LCL & Std-dev. \\
\hline \multirow{3}{*}{ LIS } & 4 & 4.7 & 4 & 4.3 & 2.66 & 0.82 & 4.6 & 3.26 & 0.67 \\
\cline { 2 - 11 } & 6 & 4.8 & 4 & 4.0 & 2.58 & 0.71 & 4.9 & 4.2 & 0.35 \\
\cline { 2 - 10 } & 8 & 4.6 & 4 & 4.0 & 3.18 & 0.41 & 4.2 & 2.76 & 0.72 \\
\cline { 2 - 10 } & 10 & 3.7 & 3 & 3.25 & 2.25 & 0.5 & 4.67 & 3.67 & 0.5 \\
\hline LIS & All & & & 4 & 2.64 & 0.68 & 4.55 & 3.07 & 0.74 \\
\hline CCC & N/A & 3.3 & 1 & 3.6 & 0.64 & 1.48 & 3.6 & 0.64 & 1.48 \\
\hline SAA & N/A & 5 & 5 & 4.2 & 2.8 & 0.7 & 4.2 & 2.8 & 0.7 \\
\hline
\end{tabular}

The coating adhesion would depend on the surface cleanliness, surface energy, and actual topology of the surface and a correlation of the coating adhesion performance to the laser processing space can be inferred by tracking these important factors. The cleaning of the Al 2024 alloy surface from the surface contaminants due to lubrication oils from prior rolling operations was observed by (Sabau, Meyer, and Leonard 2020) at a fluence of $1.24 \mathrm{~J} / \mathrm{cm}^{2}$. The XPS data shows that this laser technique is effective at removal of some surface contaminants, particularly for $\mathrm{C}$ which was modestly reduced for 2 laser pulses processing dramatically reduced for 8 laser pulses. It is expected that at the higher fluence of $1.78 \mathrm{~J} / \mathrm{cm}^{2}$ the cleaning would be even more efficient, i.e., requiring even fewer laser shots to clean the surface. Preliminary data on contact angle measurements for LIS surfaces at a fluence of $1.24 \mathrm{~J} / \mathrm{cm}^{2}$ were used to calculate the surface energies of $\sim 30 \mathrm{mN} / \mathrm{m}$ for CCC and LIS surfaces with $2,4,6$, and 8 pulses/spot and $\sim 66 \mathrm{mN} / \mathrm{m}$ for SAA. This indicate that the wetting behavior indicate that LIS surfaces were compatible with CCC and that the wetting behavior exhibited a weak variation with respect to the number laser shots per spot (all smaller than 8). Concerning the topology variation with processing, the roughness factor for LIS surfaces, $r_{L I S}$, was qualitatively evaluated in Section 3.2. $r_{\text {LIS }}$ was estimated to monotonically decrease with increasing $\mathrm{U}$; being smallest at highest speed $(10 \mathrm{~mm} / \mathrm{s}) . r_{L I S}$ is likely to exhibit a small variation with $\mathrm{U}$, especially at lower $\mathrm{U}$ values (4 to $6 \mathrm{~mm} / \mathrm{s}$ ). Moreover, $r_{L I S}$ is likely to exhibit a weak dependence on $U$ at the higher fluence considered $\left(1.78 \mathrm{~J} / \mathrm{cm}^{2}\right)$. Finally, $r_{L I S}$ is likely to be larger at the 
$1.78 \mathrm{~J} / \mathrm{cm}^{2}$ fluence than at the $1.24 \mathrm{~J} / \mathrm{cm}^{2}$ fluence. All of these findings enumerated in this paragraph indicate that CA would be likely to exhibit the following trends: (a) be higher at the lower end of the speed range considered (4 to $6 \mathrm{~mm} / \mathrm{s}$ ), (b) be lowest at the highest speed considered (10 mm/s), and (c) be higher at the $1.78 \mathrm{~J} / \mathrm{cm}^{2}$ fluence.

\subsection{EFFECT OF OPEN-TIME AND COATING THICKNESS EFFECT ON ADHESION RATING}

First, the coating adhesion rating dependence of the storage open time is presented. For each laser fluence, the cross-hatch adhesion rating data for both the $4 \mathrm{~mm} / \mathrm{s}$ and $6 \mathrm{~mm} / \mathrm{s}$ were averaged together since their ratings were close and ordered according to the open time. The cross-hatch adhesion ratings at several open times are shown in Table 26 for the two laser fluences considered in this study. The number of ratings used to obtain each reported coating adhesion average is also indicated in Table 26 . There were only few specimens at open times of 49 days and 235 days. Although there was not a dedicated effort to systematically investigate the storage open time effect, the data shows that the adhesion of primer after 200 days was still high, above 3.8 rating, and not significantly decreased compared to that for the shortest open time (20-21 days) investigated, indicating that LIS would allow extended open time for the application of CA7233 primer.

Table 26. The average cross-hatch rating for both $4 \mathrm{~mm} / \mathrm{s}$ and $6 \mathrm{~mm} / \mathrm{s}$ laser raster speeds at two fluences and several open times

\begin{tabular}{|c|c|c|c|c|}
\hline$F_{1}\left[\mathrm{~J} / \mathrm{cm}^{2}\right]$ & \multicolumn{2}{|c|}{1.238} & \multicolumn{2}{c|}{1.782} \\
\hline $\begin{array}{c}\text { Open time } \\
{[\text { day }]}\end{array}$ & $\begin{array}{c}\text { Average } \\
\text { Rating }\end{array}$ & $\begin{array}{c}\text { No. of } \\
\text { ratings }\end{array}$ & $\begin{array}{c}\text { Average } \\
\text { Rating }\end{array}$ & $\begin{array}{c}\text { No. of } \\
\text { ratings }\end{array}$ \\
\hline 20 & - & - & 4.9 & 16 \\
\hline 49 & 4.5 & 2 & - & - \\
\hline 70 & 3.8 & 11 & - & - \\
\hline 228 & 4.8 & 5 & - & - \\
\hline 235 & - & - & 3.7 & 3 \\
\hline
\end{tabular}

The results of the average X-cut and cross-hatch tests were presented in Figure 32 for all surface treatments considered and three coating thickness ranges, namely 15 to $23 \mu \mathrm{m}, 23$ to $30 \mu \mathrm{m}$, and 30 to 44 $\mu \mathrm{m}$, at a laser fluence of $1.24 \mathrm{~J} / \mathrm{cm}^{2}$. For each coating thickness range, the datapoints were connected for all surface pretreatments in order better illustrate the coating thickness dependence. The data for the 
ratings were combined for all of the coating sessions, i.e., irrespective of the open-time. The average adhesion ratings were obtained from at least two specimens per each condition.

(a)

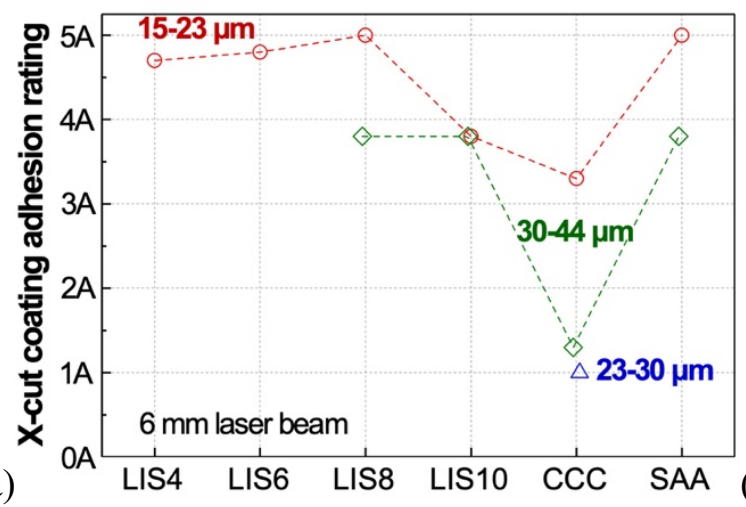

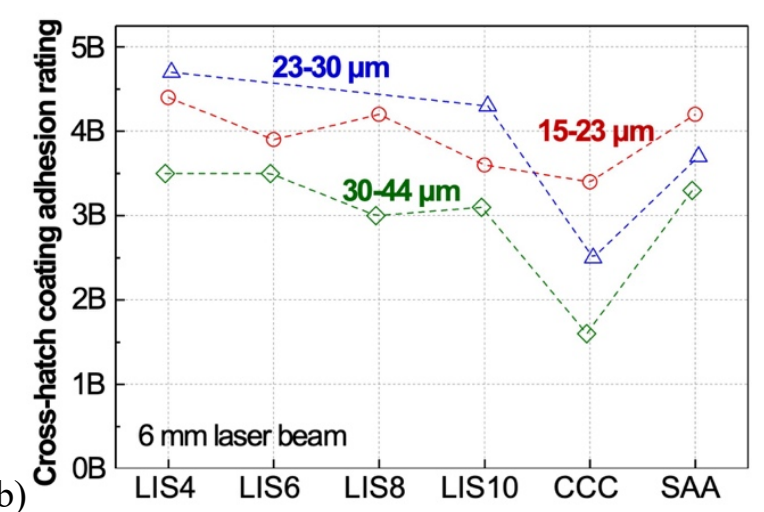

(b)

b) cross-hatch tests for different coating thickness

Based on the X-cut test results, increased coating thickness resulted in lower adhesion on CCC, SAA, and some LIS panels. Similarly, cross-hatch test results also showed lowered adhesion in the primer coating thicker than $30 \mu \mathrm{m}$ for all LIS surfaces as well as CCC and SAA surfaces. Moreover, the adhesion performance of the primer coating for the standard coated specimens (both 15-23 $\mu \mathrm{m}$ and 23-30 $\mu \mathrm{m}$ coating thickness) were not significantly different. These results are consistent with the vendor specifications that the thickness of primer coating should not exceed $30 \mu \mathrm{m}$ in order to avoid reduction of adhesion performance. 


\section{LASER TREATMENT FOR CORROSION PROTECTION}

It was reported that the surface microstructures evolved by laser-induced local melting and rapid solidification appeared to influence corrosion behavior of $\mathrm{Al}$ alloys and resulted in either increased or decreased corrosion resistance(McCafferty, Moore, and Peace 1982; McMahon et al. 1994; Li et al. 1996; Watkins, McMahon, and Steen 1997; Watkins et al. 1998). Short-pulse lasers, such as excimer lasers, were found to provide a mechanism for changing the surface morphology without causing significant damage to the underlying material (Kai et al. 2001). However, these proof-of-principle studies were conducted using laser systems that employed small laser beam sizes, e.g., between 10 to $500 \mu \mathrm{m}$, without aiming at large-scale applications.

In this study, a laser-interference structuring technique, which was developed for surface treatments of metals, polymers, and ceramics (Daniel, Mücklich, and Liu 2003; Veith et al. 2005; Sabau, Greer, et al. 2016), is considered as an alternative pretreatment for primer application. The detailed experimental setup

and the physical insights on the laser-interference structuring on metal surfaces were described in (Daniel, Mücklich, and Liu 2003; Mücklich, Lasagni, and Daniel 2005). This technique, recently referred to as Direct Laser Interference Patterning (Lasagni et al. 2011; Lasagni et al. 2015; Lasagni et al. 2017), involves the overlapping of two or more incident laser beams, which originate from a main laser beam on the specimen surface to yield a periodic power distribution on the material surface. This LIS technique using a nano-second laser (Lasagni et al. 2007; D'alessandria, Lasagni, and Mücklich 2008) was found to achieve both surface cleaning, i.e., surface contaminant removal, and surface structuring (Sabau, Meyer, and Leonard 2020). The adhesion of Desoprime ${ }^{\text {TM }}$ HS CA7233 Military Epoxy Primer on treated CCC, SAA. and LIS A12024-T3 panels were recently reported to be very similar (Sabau, Jun, and McClurg 2020), supporting the possibility of LIS as an alternative pretreatment.

\subsection{SPECIMENS FOR CORROSION TESTING}

The specimens were processed in raster mode by translating the sample while the laser fired pulses at 10 $\mathrm{Hz}$ frequency. At the end of each line scan, the specimen was then positioned straight up from last spot such that the next line scan will be laser processed. The overlap between adjacent rows of scans was 1 $\mathrm{mm}$. The scan direction was identical to that of the rolling direction. The beam angle was $12^{\circ}$, for a theoretical periodicity of the structures of $\sim 1.7 \mu \mathrm{m}$. In this work, two laser fluences of $F_{l}=1.238$ and $1.782 \mathrm{~J} / \mathrm{cm}^{2}$ per pulse were used by varying the laser spot size ( $d_{b}=6$ and $5 \mathrm{~mm}$, respectively) while keeping the same average power of $3.5 \mathrm{~W}$. The pulse repetition rate was $f_{L}=10 \mathrm{~Hz}$. To quantify the effect 
rastering speed, $U$, of the laser beam on the energy deposited on the specimen surface, the two process variables were introduced: (a) the number of pulses that a local area is exposed, $N_{P}(U)=d_{b} \cdot f_{L}$, and (b) the accumulated fluence on the specimen surface, $F_{A}=N_{P} \cdot F_{l}$, where the fluence of each shot is $F_{l} . N_{P}$ and $F_{A}$, are given in Table 27 for all LIS conditions considered in this study. The specimen labels for each LIS condition are also shown in Table 27. As the raster speed is increased, the surface is exposed to a smaller number of shots and smaller accumulated fluences. Figure 33 shows several images of the laserstructured surfaces. The effect of the raster speed is evident by the curved laser spot imprints.
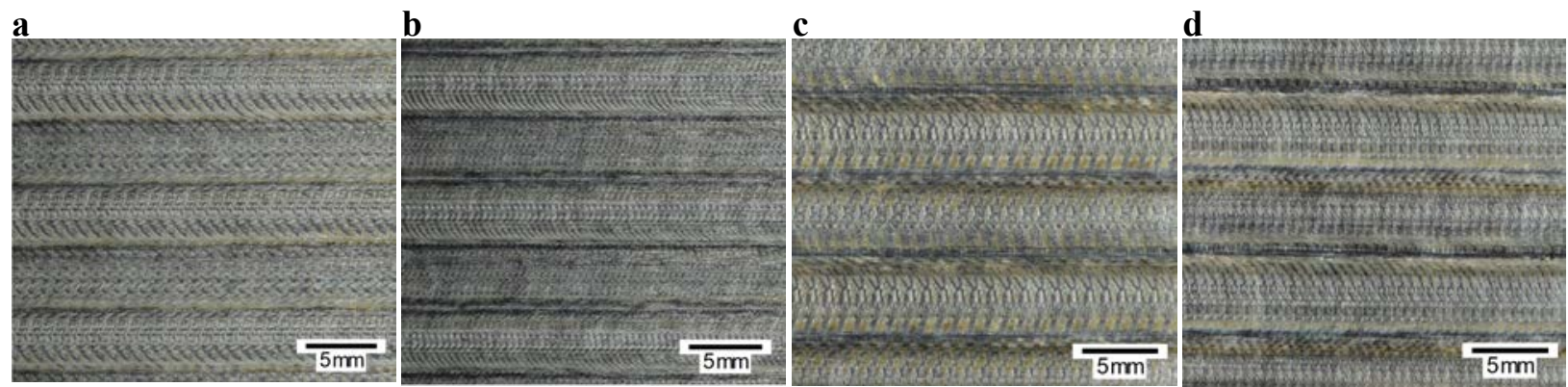

Figure 33. Laser-structured surfaces of Al 2024 panels with different raster speeds and beam spot sizes: (a) 8 $\mathrm{mm} / \mathrm{s}$ with $\emptyset=5 \mathrm{~mm}$, (b) $4 \mathrm{~mm} / \mathrm{s}$ with $\emptyset=5 \mathrm{~mm}$, (c) $10 \mathrm{~mm} / \mathrm{s}$ with $\emptyset=6 \mathrm{~mm}$ and (d) $6 \mathrm{~mm} / \mathrm{s}$ with $\emptyset=6 \mathrm{~mm}$.

Table 27. Energy metrics for several raster speeds for laser fluences $F_{1}$ of 1.782 and $1.238 \mathrm{~J} / \mathrm{cm}^{2}$ and identification of specimen process conditions (process ID)

\begin{tabular}{|c|c|c|c|c|c|c|}
\hline$F_{1}\left[\mathrm{~J} / \mathrm{cm}^{2}\right]$ & \multicolumn{3}{|c|}{1.782} & \multicolumn{3}{c|}{1.238} \\
\hline$U[\mathrm{~mm} / \mathrm{s}]$ & Sample ID & $N_{P}$ & $F_{A}\left[\mathrm{~J} / \mathrm{cm}^{2}\right]$ & $*$ Sample ID & $N_{P}$ & $F_{A}\left[\mathrm{~J} / \mathrm{cm}^{2}\right]$ \\
\hline 2 & - & - & - & LIS 2B & 30 & 37.14 \\
\hline 4 & LIS 4A & 13 & 22.28 & LIS 4B & 15 & 18.57 \\
\hline 6 & LIS 6A & 8 & 14.85 & LIS 6B & 10 & 12.38 \\
\hline 8 & - & - & - & LIS 8B & 7 & 9.28 \\
\hline 10 & LIS 10A & 5 & 8.91 & - & - & - \\
\hline
\end{tabular}

* Samples LIS 2B, 4B, 6B and 8B were acetone wiped and dry-wiped with lint-free wipes immediately after the LIS. 
A WYKO NT9100 surface profilometer was used to obtain surface roughness data for the alloy surfaces in the as-received condition, i.e., without any laser processing, and after laser-interference processing (Appendix A). Due to the space limitation, the topology, microstructure, and surface chemistry are not presented in this study as they were addressed in detail in other publications (Sabau, Jun, and McClurg 2020; Sabau, Meyer, and Leonard 2020) for laser fluences $F_{l}$ of $1.238 \mathrm{~J} / \mathrm{cm}^{2}$ and are highlighted here for the sake of completion. First, the X-ray photoelectron spectroscopy (XPS) data showed that this laser technique is effective at removal of some surface contaminants, particularly for carbon which was modestly reduced for two laser pulses processing dramatically reduced for eight laser pulses (Sabau, Meyer, and Leonard 2020). Second, the laser-interference processing was found to reduce the formation of CuMn-rich precipitates over a 500-800 nm depth from the top surface, which may lead to an increase in corrosion protection as the localized corrosion would be reduced. Due to the high energy, it is expected that at the higher fluence of $1.782 \mathrm{~J} / \mathrm{cm}^{2}$ the cleaning effect would be even more efficient, i.e., requiring even fewer laser shots to clean the surface. Based on an analytical model for the topology variation with $U$ (Sabau, Jun, and McClurg 2020), the surface topology would be the least affected at highest speed (10 $\mathrm{mm} / \mathrm{s}$ ), exhibit a small variation between $U=4 \mathrm{~mm} / \mathrm{s}$ and $U=6 \mathrm{~mm} / \mathrm{s}$, variation which would be larger at the $1.782 \mathrm{~J} / \mathrm{cm}^{2}$ fluence than at the $1.238 \mathrm{~J} / \mathrm{cm}^{2}$ fluence.

\subsection{CORROSION TESTS}

The corrosion behavior of LIS, CCC and SAA Al panels was electrochemically investigated without the primer coating to assess the corrosion resistance for each pretreatment. For electrochemical measurements, LIS Al panels with 4A and 10A process conditions (refer Table 27) were used along with $\mathrm{CCC}$ and SAA panels. Bare Al panels abraded with 600 grit SiC paper, which were referred to as ABR, were also considered as one of the reference conditions. In the first set of electrochemical measurements, a paint cell was used to expose $14.8 \mathrm{~cm}^{2}$ of panel surface, i.e., working electrode, in $0.1 \mathrm{M} \mathrm{NaCl}$ solution with a graphite rod and a saturated calomel electrode (SCE) as the counter and reference electrodes, respectively. The surface area of the graphite rod in contact with the solution was approximately $7 \mathrm{~cm}^{2}$. The area of the counter electrode was about one half of that of the working electrode. However, the measured current during anodic polarization of Al panels was not limited by the small area of the counter electrode. For this first test, a 40 min period for open circuit potential (OCP) was considered, which is longer than the 30 min OCP period that is commonly used for A12024(Ralston, Young, and Buchheit 2009; Wang, Yang, and Frankel 2017). After OCP delay for 40 min, potentiodynamic polarization was 
applied for the pretreated $\mathrm{Al}$ panels from $-0.1 \mathrm{~V}$ to $0.5 \mathrm{~V}$ with respect to the last OCP. The scan rate was $2.5 \mathrm{mV} / \mathrm{s}$.

The second set of electrochemical measurements were conducted only for the LIS and ABR panels using a similar setup used in the first set of measurements but with a much smaller working electrode area of $0.7 \times 0.7 \mathrm{~cm}^{2}$. Here, a masking tape applied to the sample surface to selectively expose the smaller working electrode area. After a 30 min OCP delay, the working electrode was polarized in either anodic or cathodic direction from the last OCP. The scan rate and final potential were $0.5 \mathrm{mV} / \mathrm{s}$ and $0.2-0.22 \mathrm{~V}$ above the OCP for the anodic polarization and $-1 \mathrm{mV} / \mathrm{s}$ and $0.4 \mathrm{~V}$ below the OCP for the cathodic polarization. All electrochemical measurements were open to air and conducted at room temperature $\left(23 \pm 1^{\circ} \mathrm{C}\right)$.

To assess the corrosion resistance of the primer-coated Al panels, ASTM B117 salt spray tests(B117-16 2016) were conducted for two batches of primer-coated Al panels summarized in

Table 28. Batch A was processed at a fluence of $F_{l}=1.782 \mathrm{~J} / \mathrm{cm}^{2}$ without any additional processing steps between laser structuring and coating application. Batch B was processed at a fluence of $F_{l}=1.238 \mathrm{~J} / \mathrm{cm}^{2}$ with additional acetone wiping right after the laser structuring. The acetone wiping consisted of two steps: (1) actual acetone wiping and (2) dry-wipe with lint-free wipes. The specific laser structuring conditions can be found in Table 27 for both batches.

Table 28. Number of panels per each condition for the ASTM B117 salt spray exposure test.

\begin{tabular}{|c|c|c|c|}
\hline Batch & $\begin{array}{c}\text { Surface } \\
\text { preparation }\end{array}$ & $\begin{array}{c}\text { Primer coating thickness } \\
\text { (min./mean/max./standard deviation) } \\
{[\mu \mathrm{m}]}\end{array}$ & $\begin{array}{c}\text { Number of } \\
\text { panels }\end{array}$ \\
\hline \multirow{5}{*}{ A } & LIS 4A & \multirow{3}{*}{$14 / 20 / 32 / 4.7$} & 4 \\
\hline & LIS 6A & & 4 \\
\hline & LIS 10A & & 4 \\
\hline & $* \mathrm{CCC}$ & $29 / 32 / 34 / 2.4$ & 4 \\
\hline & $* \mathrm{SAA}$ & $26 / 28 / 32 / 2.6$ & 4 \\
\hline \multirow{4}{*}{$* * \mathrm{~B}$} & LIS 2B & \multirow{4}{*}{$9 / 13 / 18 / 2.8$} & 6 \\
\hline & LIS 4B & & 6 \\
\hline & LIS 6B & & 6 \\
\hline & LIS 8B & & 6 \\
\hline
\end{tabular}

*rinsed with ethanol and dry-wiped with lint-free wipes prior to coating.

**Wiped with acetone and dry-wiped with lint-free wipes immediately after LIS. 
Prior to the salt spray tests, X-scribes were made on the diagonals of coated panels using a box cutter to expose the Al substrate. The diagonal cuts fully penetrated the coating. The edges of all the panels were masked with beeswax. The panels were then placed on horizontal racks in the salt spray chamber. Panels were slightly inclined from the vertical direction by approximately $30^{\circ}$. To prepare $3.5 \mathrm{wt} . \% \mathrm{NaCl}$ solution reservoir and produce the inlet steam, tap water was used instead of $4 \mathrm{M} \Omega$ grade water. The quality report of tap water used in this work is given in the Appendix B. Salt spray tests experienced multiple off-times at every 120-140 h exposure due to a setup limitation. Each off-time lasted approximately $40-50 \mathrm{~h}$ in most cases. During the off-time period, photo images of the coated panels were taken without removing the panels from the test chamber. The pictures of these "wet" panels were used to track the evolution of the corrosion damage, such as blister appearance and corrosion on scribe. The creepage rating was assessed according to ASTM D1654 after the specimens were taken from the salt spray chamber, cleaned, and imaged.

\subsection{RESULT AND DISCUSSION FOR CORROSION TESTING}

\subsubsection{Electrochemical measurement results for uncoated specimens}

The potentiodynamic curves of SAA, CCC, ABR and LIS (4A and 10A) panels, collected in the first set, are shown in Figure 34. SAA panel exhibited noisy passive current from the initial potential, i.e., $0.1 \mathrm{~V}$ below OCP, to $-0.25 \mathrm{~V}_{\text {SCE. }}$. The sharp current increase is considered to indicate a local insulation failure of the anodized layer (Figure 34a). Meanwhile, the CCC, ABR and LIS 4A, and LIS 10A panels showed cathodic and anodic currents with increasing potential and zero current potential $\left(E_{\mathrm{zc}}\right)$ which distinguished the anodic and cathodic regions. CCC panel showed lower cathodic current compared to that of the ABR panel in the cathodic region due to the cathodic inhibition of chromates. Interestingly, both LIS panels showed some reduction in cathodic current with respect to that of the ABR panel (both LIS and ABR did not have any cathodic inhibitors). One possibility to account for the decreased cathodic current is that laser structuring reduced Cu-rich intermetallics (Sabau, Meyer, and Leonard 2020) that are favorable cathodic reaction sites.

Once $E_{\mathrm{zc}}$ was passed, uninterrupted increase in anodic current was observed with increasing potential in ABR, LIS, and CCC panels, indicating the spontaneous breakdown of passivity (Lopez-Garrity and Frankel 2014a, 2014b; Wang et al. 2013; Wang et al. 2014; Wang et al. 2015; Seong et al. 2015). Spontaneous breakdown, which is evidenced by localized attacks, is different from active corrosion, 
which is characterized by uniform corrosion. For the spontaneous breakdown cases, the breakdown potential $\left(E_{\mathrm{b}}\right)$ was defined as the potential at which the current density reached $0.7 \mu \mathrm{A} \cdot \mathrm{cm}^{-2}$ (Figure $34 \mathrm{~b}$ ). The anodic current on CCC panel increased slower than ABR and LIS panels likely due to anodic inhibition of hexa-chromates. This result somewhat contradicts several studies, in which it was asserted that chromates had no effect on inhibiting the rate of localized dissolution in Al alloys (Sehgal, $\mathrm{Lu}$, and Frankel 1998; Sehgal et al. 2000). Thus, a reasonable explanation would be that chromates did not retard the growth of $\mathrm{Al}$ pitting but did decrease the frequency of pit initiation. The anodic currents increased uninterrupted on ABR and LIS panels, implying that the laser structuring did not suppress pitting initiation and propagation in $\mathrm{Al} 2024$.

$\mathbf{a}$

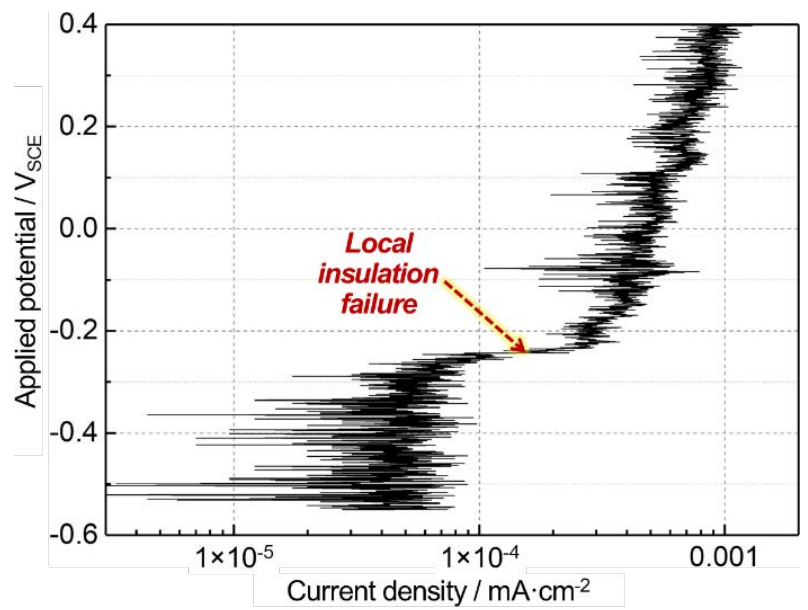

b

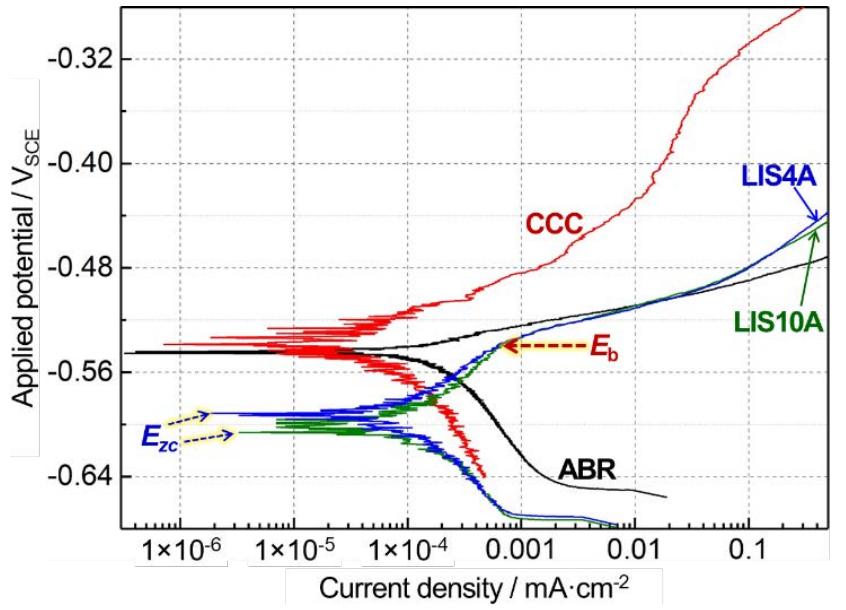

Figure 34. Potentiodynamic curves of (a) a SAA specimen and (b) ABR, CCC, and two LIS specimens at a fluence of $F 1=1.782 \mathrm{~J} / \mathrm{cm}^{2}$ per pulse (LIS 4A and LIS 10A) obtained with a working electrode area of 14.8 $\mathbf{c m}^{2}$. Zero current and breakdown potentials are denoted as $E_{z c}$ and $E_{b}$, respectively. For the SAA specimen, a sharp current increase is considered to indicate a local insulation failure.

Values of $E_{\mathrm{b}}, E_{\mathrm{zc}}$, and the cathodic current at the potential $50 \mathrm{mV}$ below $E_{\mathrm{zc}}, i_{\mathrm{c}-50}$, from the first set polarization measurement are shown in Figure 35. $i_{\mathrm{c}-50}$ was used for a quantitative comparison of the cathodic reaction rates in $\mathrm{ABR}, \mathrm{CCC}$, and LIS surfaces. $E_{\mathrm{zc}}$ was about $40 \mathrm{mV}$ lower in LIS surfaces than the others. $E_{\mathrm{b}}$ values of LIS and ABR panels were similar to each other and lower than the $E_{\mathrm{b}}$ values of CCC panels. This suggests that LIS in this work had no observable effect on Al passivity. The values of $i_{\mathrm{c}-50}$ on LIS panels were about one half of that of the ABR panels but 2-2.5 times of that of the CCC panels, confirming that LIS surface reduced cathodic reaction in Al 2024 but not as much as CCC treatment did. 

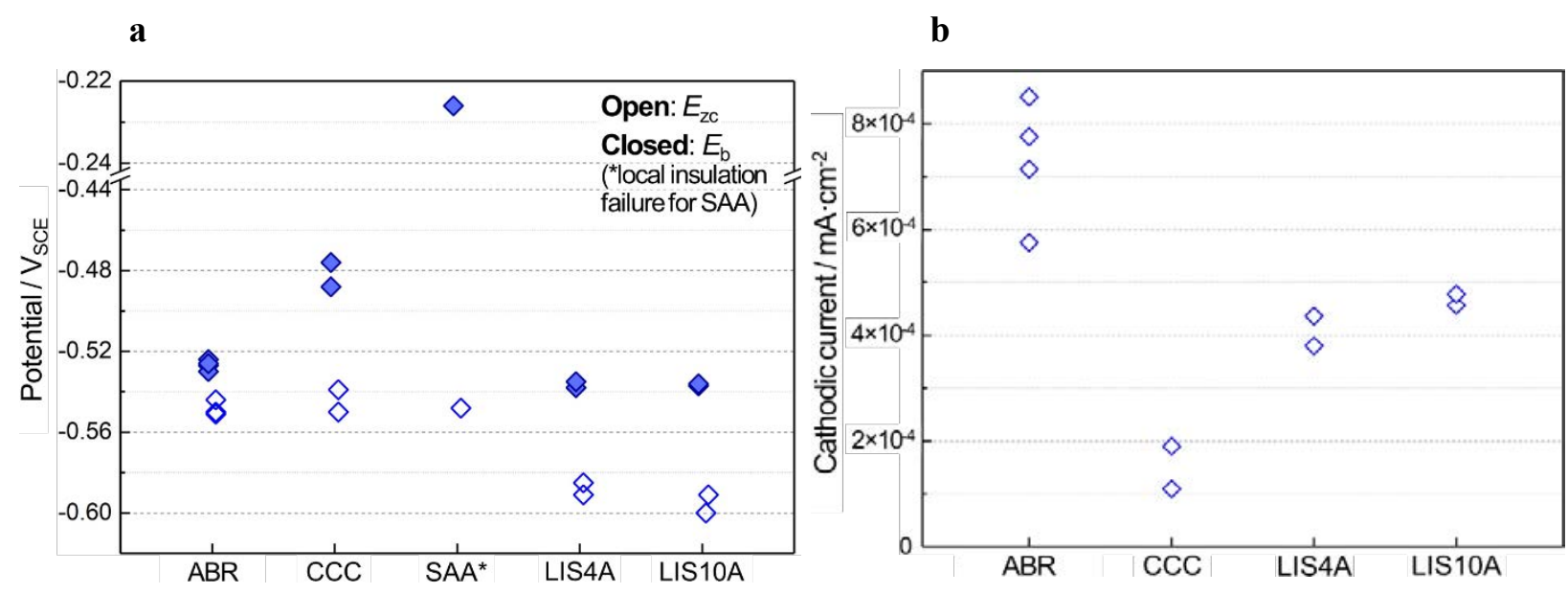

Figure 35. Comparison of (a) $E_{z c}$ and $E_{b}$ values and (b) $i_{c-50}$ values for ABR, CCC, and two LIS specimens at a fluence of $F_{1}=1.782 \mathrm{~J} / \mathrm{cm}^{2}$ per pulse (LIS $4 \mathrm{~A}$ and LIS 10A).

In the second set of electrochemical measurements, the anodic and cathodic polarizations were separately conducted for LIS (4A and 10A) and ABR panels with a much smaller working electrode area $\left(0.49 \mathrm{~cm}^{2}\right)$ than that of the first set measurements $\left(14.8 \mathrm{~cm}^{2}\right)$ to rule out any possible measurement artifact due to the use of a very large working electrode area. The anodic and cathodic curves in the second set are presented in Figure 36. A relatively fast increase of the current associated with passivity breakdown was observed for all panels around $-0.53 \mathrm{~V}_{\mathrm{SCE}}$ in the anodic curves (Figure 36a), implying that there was no or only little difference in corrosion resistance between ABR and LIS Al panels. This agrees well with the results from the first set measurement. LIS (10A) panels showed noisy current fluctuation before the breakdown, which is considered as metastable pitting behavior commonly observed in passive alloys (Jun 2016; Jun, Holguin, and Frankel 2013).

Meanwhile, the comparison of cathodic curves showed that the current decreased in the following order: ABR, LIS (10A) and LIS (4A) panels (Figure 36b). It is again confirmed that the cathodic reaction rate is lower in LIS than ABR panels. However, different cathodic reaction rates for the LIS (10A) and LIS (4A) were observed in the second set measurement, differences which were not captured in the first set measurement (Figure 34b and Figure 35b). One explanation for the difference in the current density for LIS (10A) and (4A) panels is that the slower laser structuring $(4 \mathrm{~mm} / \mathrm{s})$ was more effective removing the $\mathrm{Cu}$-rich intermetallics because the laser energy input per area was higher for the lower raster speed, yielding higher local temperatures likely causing more intensive solutionizing heat treatment near the treated surface. 
a

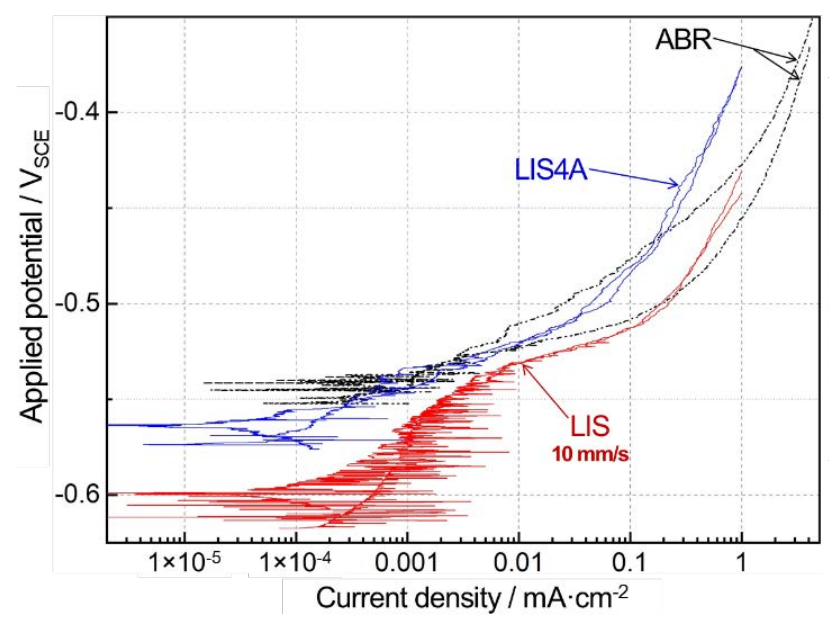

b

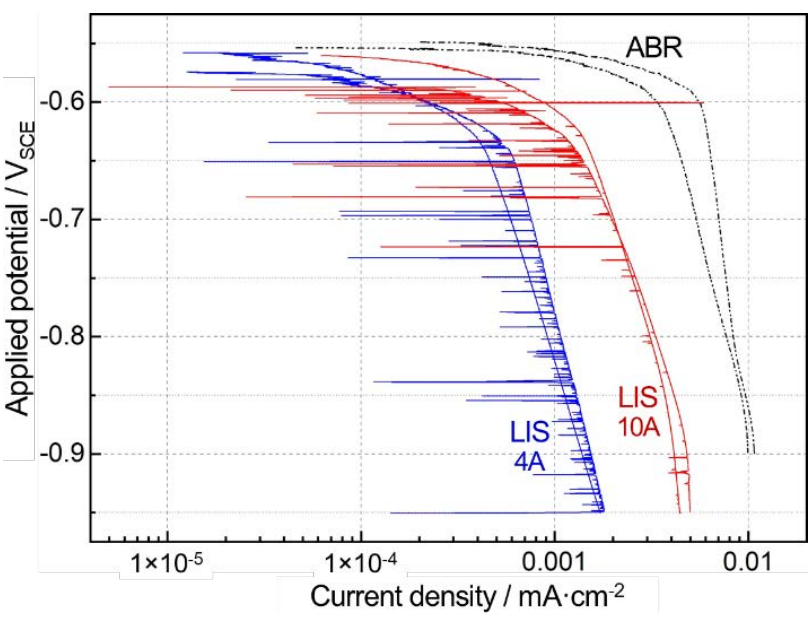

Figure 36. Potentiodynamic (a) anodic curves and (b) cathodic curves of ABR, CCC, and two LIS specimens at a fluence of $F_{1}=1.782 \mathrm{~J} / \mathrm{cm}^{2}$ per pulse (LIS4A and LIS10A) obtained with a working electrode area of 0.49 $\mathbf{c m}^{2}$.

\subsubsection{Salt spray test results for batch A of coated specimens}

The corrosion damage of primer-coated panels after the salt spray exposure tests is presented in this section. The surface images after $500 \mathrm{~h}$ and $1000 \mathrm{~h}$ exposure are shown in Figure 37, Figure 38, and Figure 39 for primer coated CCC, SAA, and LIS panels in the batch A, respectively. The coated CCC and SAA panels exhibited minor corrosion damage along the scribes with no significant differences between the 500 and $1000 \mathrm{~h}$ exposures (Figure 37). The coated LIS panels, i.e. LIS (4A), (6A) and (10A), showed one blister spot per each specimen on/near the scribes after 500 and $1000 \mathrm{~h}$ exposure (Figure 38 and Figure 39). 
$\mathbf{a}$

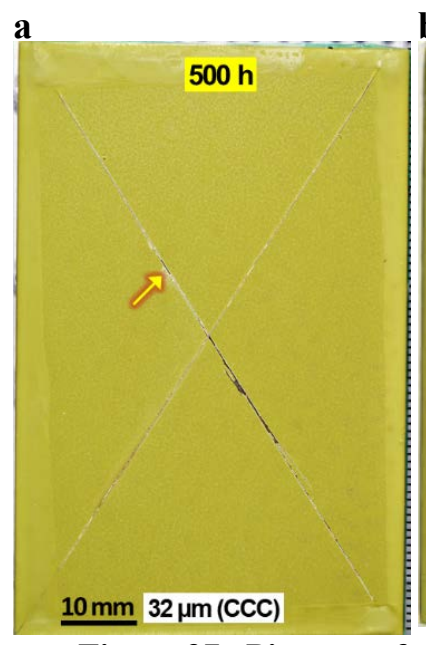

b

Figure 37. Pictures of coated panels with CCC $(a, b)$ and SAA $(c, d)$ pre-treatments indicating minor corrosion damage after $500 \mathrm{~h}$ or $1000 \mathrm{~h}$ exposures.

a

$10 \mathrm{~mm} 20 \mu \mathrm{m}$ (LIS 4A)

b

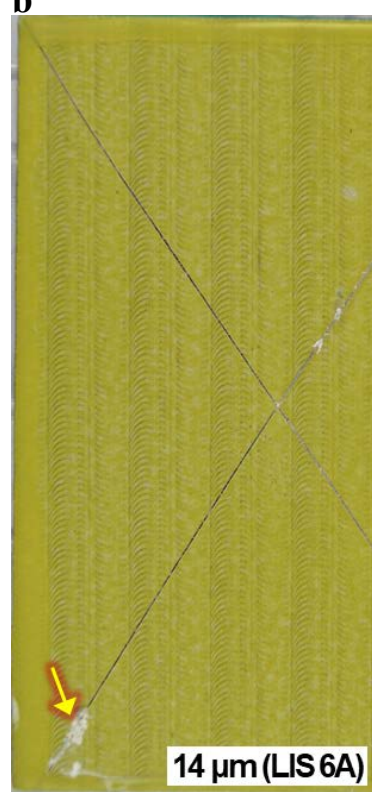

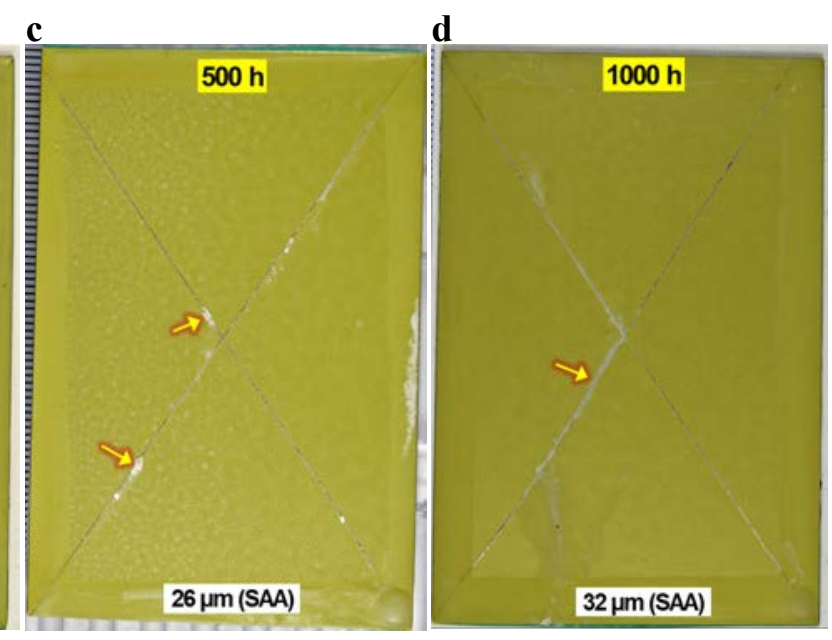

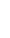



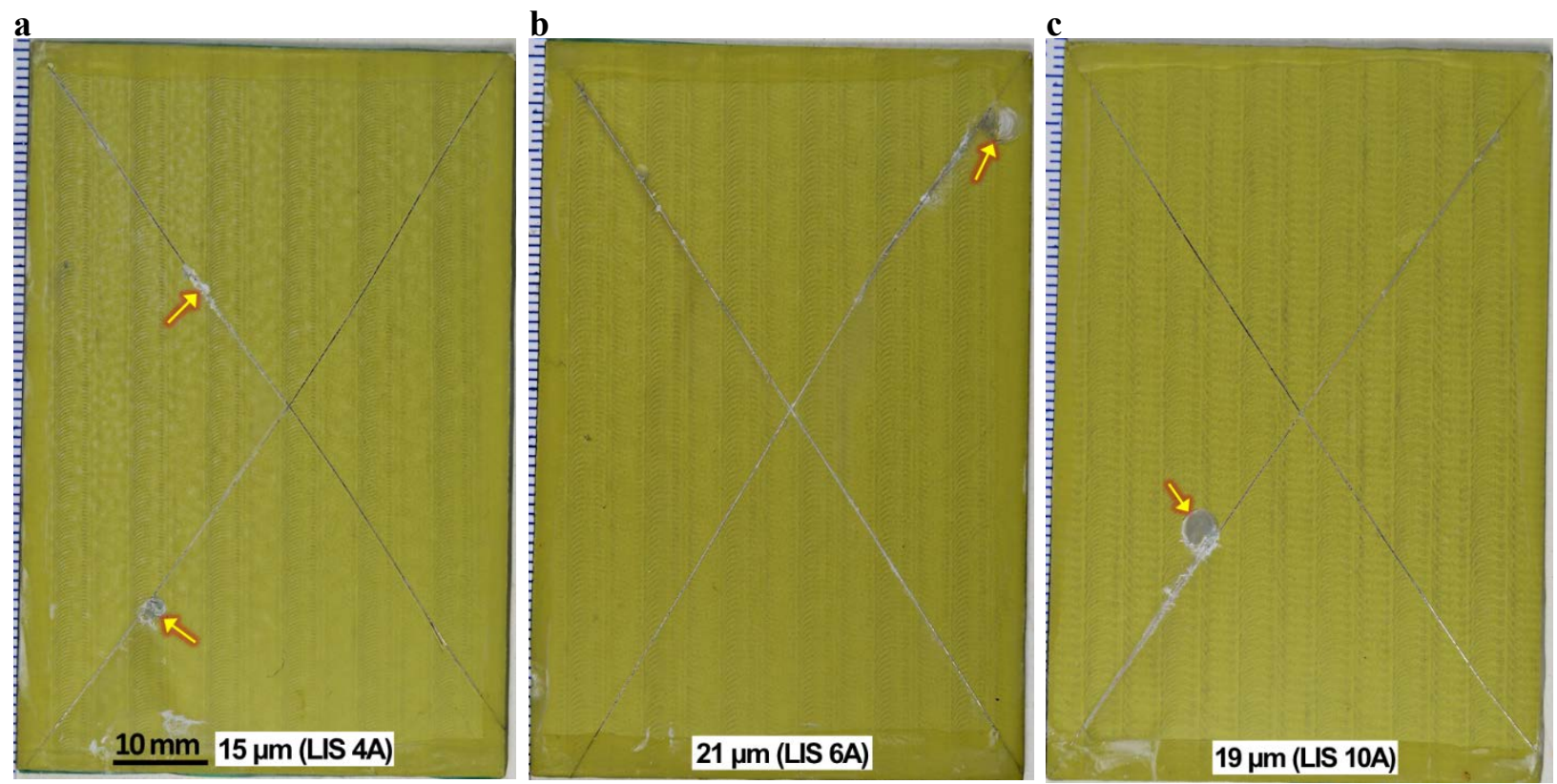

Figure 39. Pictures of selected LIS test panels with the most corrosion damage after $1000 \mathrm{~h}$ exposure for conditions: (a) LIS 4A, (b) LIS 6A, and (c) LIS 10A. LIS A batch was processed at a fluence of $F_{1}=1.782$ $\mathbf{J} / \mathrm{cm}^{2}$ with no additional wiping after LIS.

The inspection after $96 \mathrm{~h}$ exposure indicated that all coated LIS panels from batch A developed only one very small blister. However, the growth of these blisters was insignificant with the salt spray exposure. As an example, pictures taken after exposures of 96, 208, 500, and $1000 \mathrm{~h}$ were used to illustrate the evolution of corrosion damage and blister growth is shown for two coated panels in Figure 40 and Figure 41. There were several (three to five) tiny blisters developed per each specimen, but only one blister grew to $\sim 5 \mathrm{~mm}$ in size. This evidence presented in Figure 38, Figure 39, Figure 40, and Figure 41 indicates that the primer-alloy interface (LIS fluence $F_{l}=1.782 \mathrm{~J} / \mathrm{cm}^{2}$ without additional acetone wiping) is susceptible to blister formation. The creepage rating was assessed for the post-exposure specimens. One specimen per each condition was taken out from the salt spray chamber after $500 \mathrm{~h}$ of exposure and three specimens per each condition were taken out from the salt spray chamber after 1,000 h of exposure. The ASTM D1654 creepage rating for all coated panels (CCC, SAA, and LIS) in batch A was larger than nine after both 500 and $1000 \mathrm{~h}$ exposure. In the batch A, the coated LIS panels exhibited the similar creepage rating with the CCC and SAA panels, but the overall corrosion resistance is considered lower due to the blister susceptibility observed in the LIS panels. 

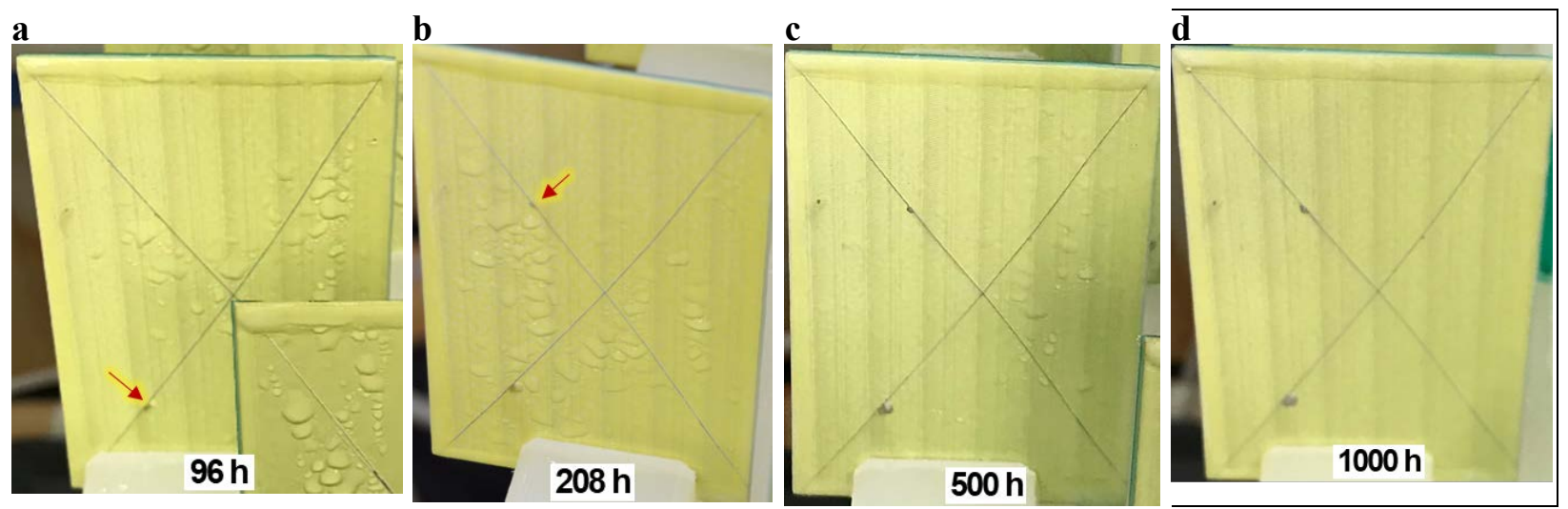

Figure 40. Evolution of minor blister growth during ASTM B117 salt-spray test for the same panel $(5.08 \mathrm{~cm} \times$ $7.62 \mathrm{~cm}$ ) with processing condition LIS $4 \mathrm{~A}$ and coating thickness $15 \mu \mathrm{m}$.
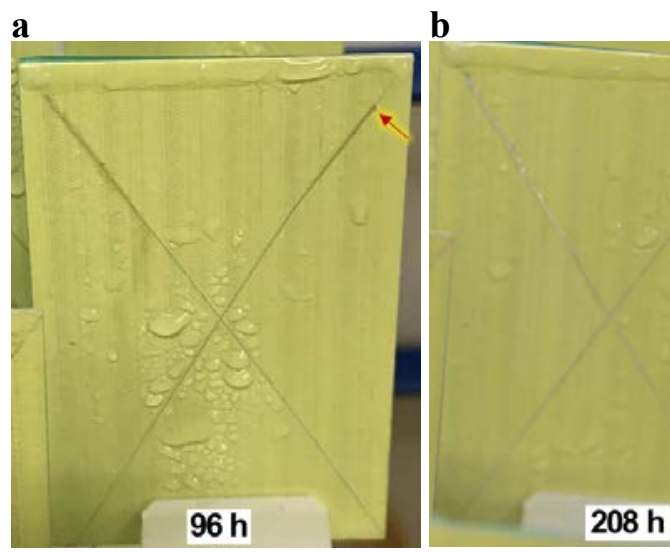

c

d

Figure 41. Evolution of minor blister growth during ASTM B117 salt-spray test for the same panel (5.08 $\times$ $7.62 \mathrm{~cm}$ ) with processing condition LIS2A and coating thickness $21 \mu \mathrm{m}$.

\subsubsection{Salt spray test results for batch B of coated specimens}

In an attempt to mitigate the blister susceptibility observed in batch A, the LIS panels in batch B were prepared by using an additional acetone wiping step right after laser structuring, with all the other processing steps similar to those for batch $\mathrm{A}$. However, for batch $\mathrm{B}$, the $\mathrm{Al}$ panels were laser processed at a fluence of $F_{l}=1.238 \mathrm{~J} / \mathrm{cm}^{2}$, lower than that used for the batch A. After an initial exposure to salt spray for $409 \mathrm{~h}$, the coated LIS panels from the batch B were kept in a non-corrosive atmospheric environment for five months. The surface images of some representative panels after $409 \mathrm{~h}$ exposure are shown in Figure 42. Neither blister formation nor corrosion at scribes was found on the panels shown and all of the 
others that are not shown, indicating that the corrosion resistance of coated LIS panels improved by an immediate acetone cleaning conducted after laser structuring. Note that all of the coated LIS panels in the batch A formed blisters within $96 \mathrm{~h}$ salt spray exposure while the batch B panels had none for $409 \mathrm{~h}$.

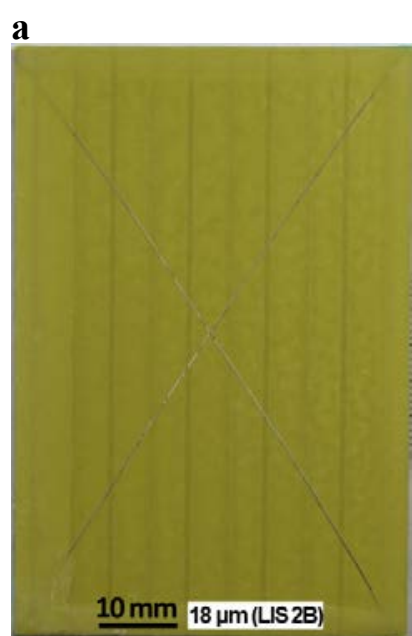

b
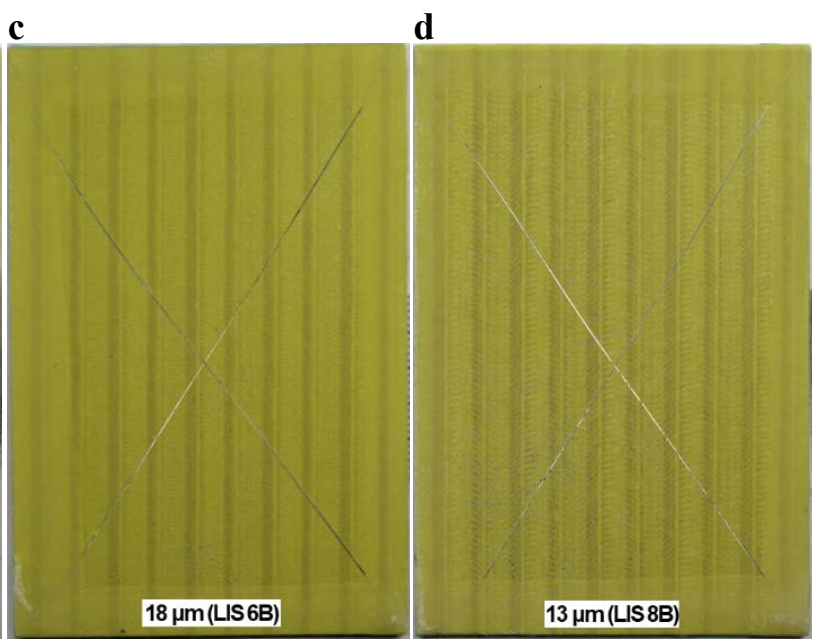

Figure 42. Pictures of selected LIS test panels showing no corrosion damage after $409 \mathrm{~h}$ exposure for the conditions: (a) LIS 2B, (b) LIS 4B, (c) LIS 6B, and (d) LIS 8B. The second batch was processed at a fluence of $F_{1}=1.238 \mathrm{~J} / \mathrm{cm}^{2}$ and acetone wiped right after LIS.

After the initial $409 \mathrm{~h}$ exposure and storage for five months, the second batch panels were re-exposed in salt spray environment for $1654 \mathrm{~h}$ to further investigate corrosion performance. After the total exposure time of $2063 \mathrm{~h}(409 \mathrm{~h}+1654 \mathrm{~h})$, the images of the coated panels with the most corrosion damage are shown in Figure 43. The formation of blister was limited to one if any, and the corrosion at the scribes appeared minor. For most LIS panels in the batch B, neither blisters nor any other form of corrosion damage was observed, as shown in Figure 44. The coated LIS panels in the batch B were inspected for corrosion damage multiple times between 505 and $2063 \mathrm{~h}$, without taking the specimens out of the test chamber. An example of corrosion damage evolution pictures taken after exposures of 790, 890, 945, and $1183 \mathrm{~h}$ for one coated panel are shown in Figure 45. For this specimen, a tiny, i.e., less than $1 \mathrm{~mm}$ in size, blister-like feature was found at each of these inspection times, without any additional growth till the end of the exposure. 
(a)

(b)

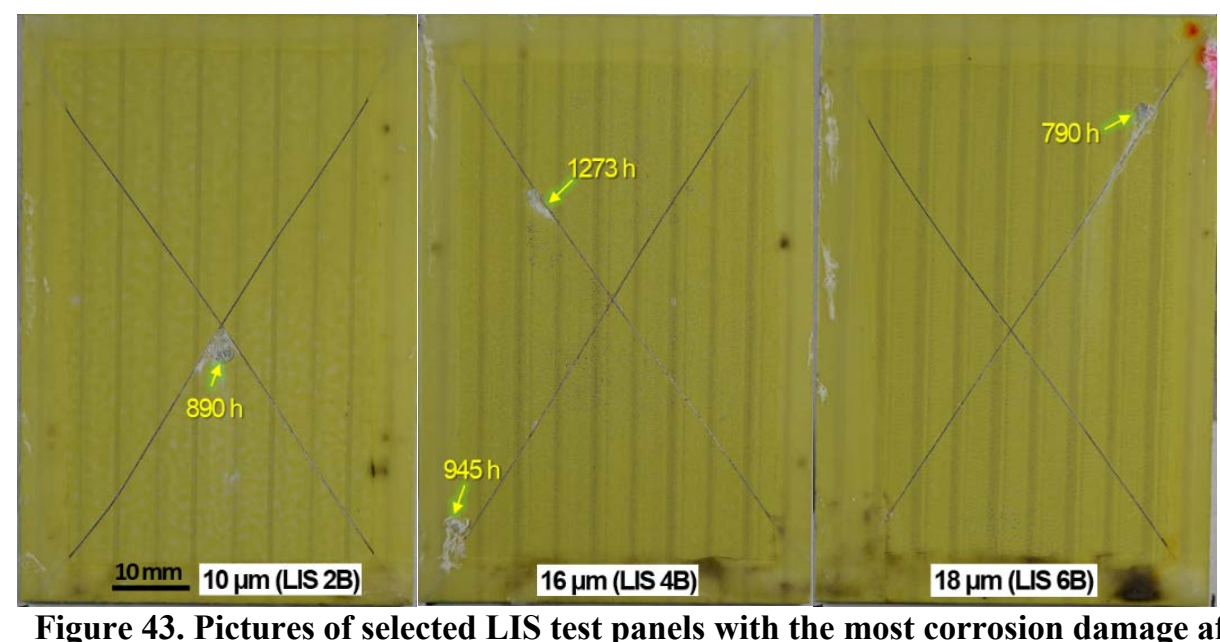

(c)

(d)

conditions: (a) LIS2B, (b) LIS4B, (c) LIS6B, and (d) LIS8B. The second batch was processed at a fluence of

$F 1=1.238 \mathrm{~J} / \mathrm{cm}^{2}$ and acetone wiped right after LIS. The initial appearance of blisters at the off-time inspection is presented in Figure 45 for each coated LIS panel in (a-d).

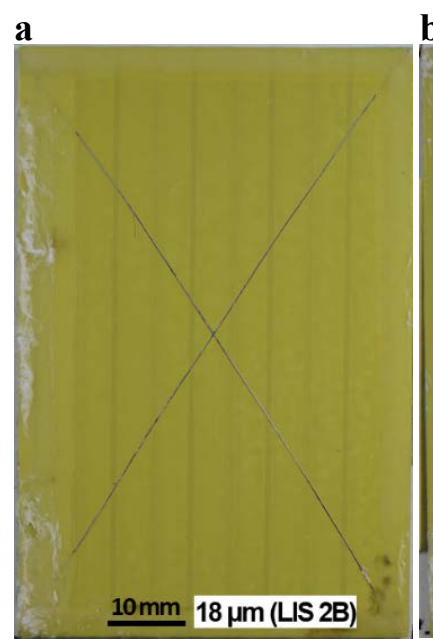

b

c

d

Figure 44. Pictures of selected LIS test panels with the least corrosion damage after $2063 \mathrm{~h}$ exposure for the conditions: (a) LIS2B, (b) LIS4B, (c) LIS6B, and (d) LIS8B. The second batch was processed at a fluence of $F 1=1.238 \mathrm{~J} / \mathrm{cm}^{2}$ and acetone wiped right after LIS. 
(a)

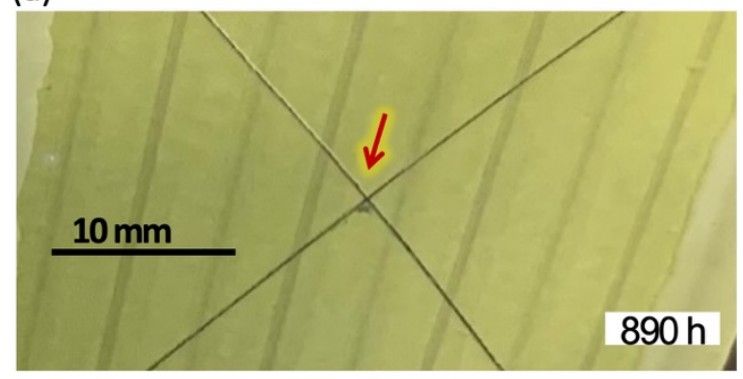

(c)

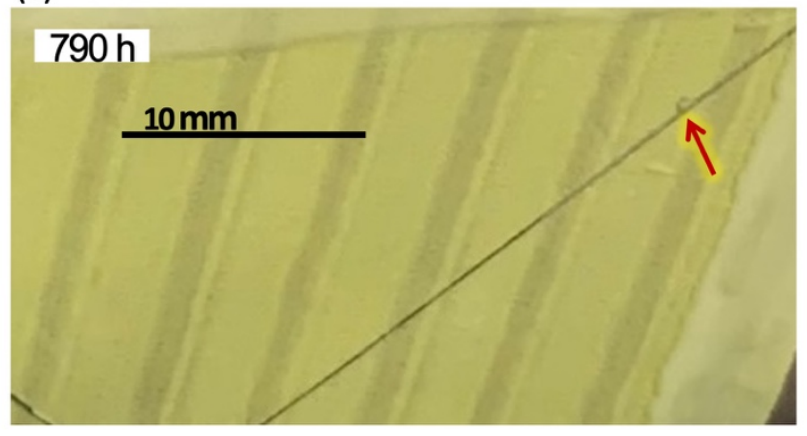

(b)

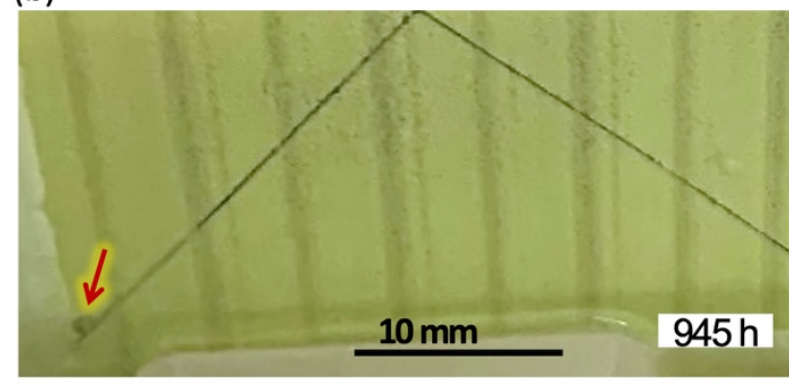

(d)

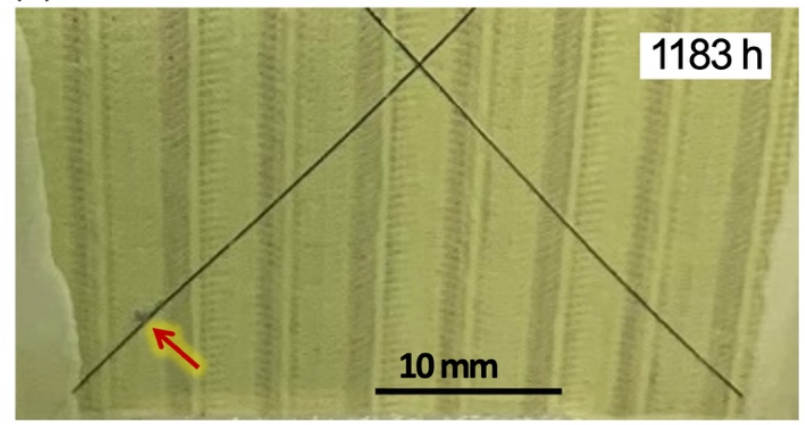

Figure 45. Evolution of the initial blister during ASTM B117 salt-spray test for the coated panels $(5.08 \times 7.62$ $\mathrm{cm}^{2}$ ) (a-d) in this figure correspond to (a-d) in Figure 43.

Table 29 summarizes the details of all individual coated panels that developed corrosion damage and blister(s) along with the blister initiation time, $t_{l}$, which is defined as the shortest time to observe blisters from the intermittent inspection times. These surface inspections indicated that the coated LIS panels developed blister-like features after $790 \mathrm{~h}$ and longer exposure. Approximately $50 \%$ of corrosion damage was found to be very minor (e.g., Figure $43 \mathrm{~d}$ showing less than $1 \mathrm{~mm}$ creepage/blister), but some coated panels developed more pronounced corrosion damage (e.g., Figure 43a, b, c with $5 \mathrm{~mm}$ creepage/blister). For the panels with no apparent corrosion damage, N/A was marked in the $t_{I}$ column. Overall, LIS (4B) coated panels exhibited the least corrosion damage with the lowest number of panels with blister formation (33\%). LIS (2B) and LIS (6B) coated panels appeared to have similar corrosion performance ranking with regard to the blister formation rate and similar initiation times. LIS (8B) coated panels exhibited more severe corrosion damage than the other LIS conditions as evidenced by the highest blister formation rate (66\%). The ASTM D1654 creepage ratings (without considering blisters) were at least nine for all coated panels in the batch $\mathrm{B}$, which is similar to the batch A. 
Table 29. Blister initiation time ( $\left.t_{\mathrm{I}}\right)$ for the coated panels in batch $B$ with the percentage of blistered panels per each LIS condition. Refer to Table 27 for the details of LIS conditions

\begin{tabular}{|c|c|c|c|c|c|c|c|c|}
\hline Condition & LIS (2B) & & LIS (4B) & & LIS (6B) & & LIS (8B) & \\
\hline \multirow{7}{*}{ Panels } & Sample ID & $t_{I}[\mathrm{~h}]$ & Sample ID & $t_{I}[\mathrm{~h}]$ & Sample ID & $t_{I}[\mathrm{~h}]$ & Sample ID & $t_{I}[\mathrm{~h}]$ \\
\hline & L2a & 790 & L4a & $\mathrm{N} / \mathrm{A}$ & L6a & $\mathrm{N} / \mathrm{A}$ & L8a & 1887 \\
\hline & $\mathrm{L} 2 \mathrm{~b}$ & N/A & $\mathrm{L} 4 \mathrm{~b}$ & 945 & L6b & N/A & $\mathrm{L} 8 \mathrm{~b}$ & N/A \\
\hline & L2c & $\mathrm{N} / \mathrm{A}$ & $\mathrm{L} 4 \mathrm{c}$ & $\mathrm{N} / \mathrm{A}$ & L6c & 1273 & L8c & 790 \\
\hline & L2d & $\mathrm{N} / \mathrm{A}$ & L4d & 1759 & L6d & 790 & L8d & $\mathrm{N} / \mathrm{A}$ \\
\hline & L2e & 890 & $\mathrm{~L} 4 \mathrm{e}$ & N/A & L6e & 790 & L8e & 1345 \\
\hline & L2f & 945 & L4f & N/A & L6f & N/A & L8f & 1183 \\
\hline $\begin{array}{l}\text { Percentage } \\
\text { of specimens } \\
\text { with blisters }\end{array}$ & \multicolumn{2}{|c|}{50} & \multicolumn{2}{|c|}{33} & \multicolumn{2}{|c|}{50} & \multicolumn{2}{|c|}{66} \\
\hline
\end{tabular}

Aside from the corrosion damage analysis on the coated panels, it could be worthwhile to review the coating adhesion as it can indirectly impact the corrosion protection(Funke 1985). In an attempt to understand the corrosion behavior presented in this study, the effect of LIS processing on coating adhesion of LIS surfaces is briefly reviewed. The coating adhesion depends on the surface cleanliness, surface energy, and actual topology of the surface. Concerning the topology variation with $U$ raster speed, the roughness factor for LIS surfaces, $r_{L I S}$, which was defined as the ratio of the LIS solid surface area to the original and unprocessed surface area, was qualitatively shown to monotonically decrease with increasing $U$ (Sabau, Jun, and McClurg 2020), being the smallest at the highest speed $(10 \mathrm{~mm} / \mathrm{s})$, exhibiting a small variation between $U=4 \mathrm{~mm} / \mathrm{s}$ and $U=6 \mathrm{~mm} / \mathrm{s}$, and becoming larger at $1.782 \mathrm{~J} / \mathrm{cm}^{2}$ fluence than at $1.238 \mathrm{~J} / \mathrm{cm}^{2}$ fluence. Based on this variation of the surface roughness by LIS and the results of coating adhesion testing, the adhesion of coating exhibited the following trends: (a) was higher at the lower end of the speed range considered (4 to $6 \mathrm{~mm} / \mathrm{s}$ ), (b) was lowest at the highest speed considered $(10 \mathrm{~mm} / \mathrm{s})$, and (c) was higher at the $1.782 \mathrm{~J} / \mathrm{cm}^{2}$ fluence than at the $1.238 \mathrm{~J} / \mathrm{cm}^{2}$ fluence. Thus, the fact that better corrosion protection was observed at raster speeds of 4 and $6 \mathrm{~mm} / \mathrm{s}$ is consistent with the trend observed for the coating adhesion. Moreover, one would expect that corrosion protection would be better at the higher fluence than at the lower fluence considered. The fact that the corrosion protection was better at the lower fluence (with additional acetone wiping) than that at higher fluence (without acetone wiping), highlights even more the importance of the additional acetone wiping right after LIS.

In summary, a comparative assessment of the coated LIS panels in the batches A and B clearly indicates the importance of immediate acetone wiping after laser structuring on suppressing and delaying the blister 
formation. It is presumed that the immediate cleaning removed any organic and inorganic surface contaminants that remained even after the laser structuring. Thus, for the sake of completion, future work may characterize the LIS surface with and without the immediate cleaning (Sabau, Meyer, and Leonard 2020).

\subsubsection{Results for roughness parameters: corrosion paper}

For the sake of completion, the roughness is characterized for the specimens used for corrosion testing. A magnification of 50X was used to image a profiling area of $130 \mu \mathrm{m} \times 174 \mu \mathrm{m}$, which was located along the centerline of a laser scan. The 3D height distributions of typical surfaces in the as-received (unprocessed condition) and after laser-interference processing are shown in Figure 46. All the imaged areas were identical in size $(130 \times 174 \mu \mathrm{m})$. The surface profiles for the as-received surfaces are shown in Figure 46(a, b) for two specimens. The surface profiles are shown in Figure 46 for laser scanning speeds of 4, 6, 8, and $10 \mathrm{~mm} / \mathrm{s}$ in Figure 46(c, d, e, f), respectively. As evidenced by Figure 46(a, b), the as-received and unprocessed aluminum surfaces are not smooth at all, exhibiting grooves in the rolling direction, quite a few microcracks, and pinholes. The laser scan direction was identical to that of the rolling direction (i.e., from the lower left corner to the upper right corner of the rotated image). The structuring induced by the laser-interference is evidenced by the very fine striations in the vertical direction, i.e., normal to the rolling direction (Figure 46(c, d, e, f)). In spite of the relatively rough original surface, the structuring by the laser-interference is evident. 
(a)
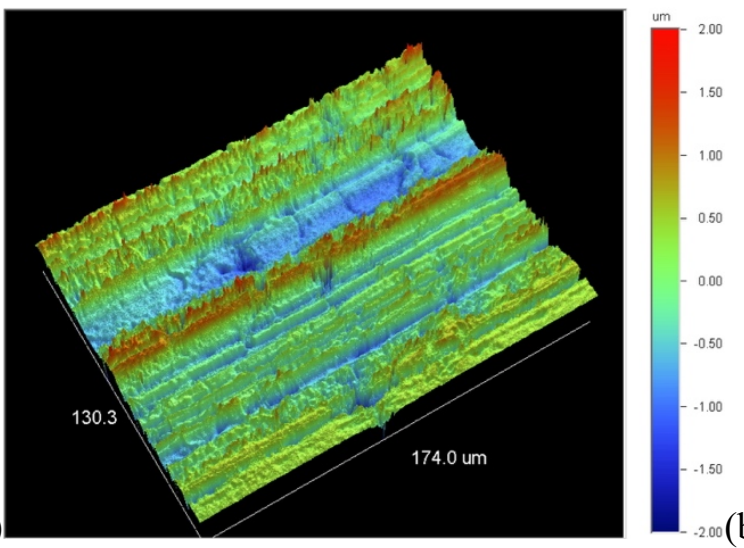

(c)

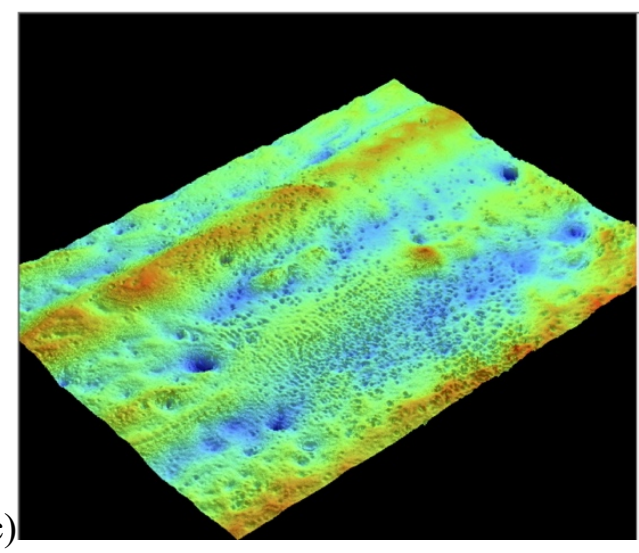

(e)

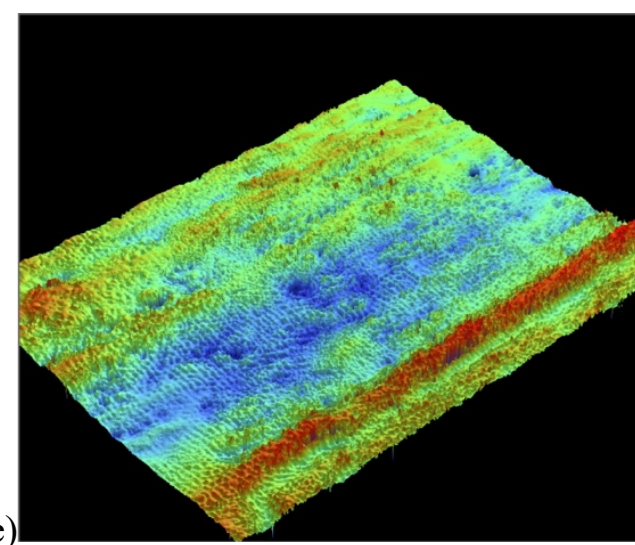

Figure 46. Surface profiles at a laser fluence of $\mathrm{F} 1=1.782 \mathrm{~J} / \mathrm{cm} 2$ per pulse for $(\mathrm{a}, \mathrm{b})$ the as-received specimens and in typical centerlines of a laser scan for raster speeds of: (c) $4 \mathrm{~mm} / \mathrm{s}$, (d) $6 \mathrm{~mm} / \mathrm{s}$, (e) $8 \mathrm{~mm} / \mathrm{s}$, and (f) 10 $\mathbf{m m} / \mathbf{s}$.

Although the roughness measurements cannot be used as a sole metric to quantify the laser-interference structuring effect, they are presented here for the sake of completion. The average roughness, or arithmetical mean deviation of the roughness profile, $\mathrm{R}_{\mathrm{a}}$, root-mean-square deviation of the roughness profile, $\mathrm{R}_{\mathrm{q}}$, and maximum height of the roughness profile, $\mathrm{Rt}$, are shown in Table 30 . As the height of the 
laser-interference structuring is expected to be less than 1.5 microns (Sabau, Meyer, and Leonard 2020), while the $\mathrm{R}_{\mathrm{t}}$ is on the order of 4 to $6 \mu \mathrm{m}$, the $\mathrm{R}_{\mathrm{t}}$ is expected to be the least affected by the laser structuring. An increase in all surface roughness metrics can be observed between the indicators measured for the laser structured specimens with respect to the indicators measured for the as-received specimens, i.e., without any laser structuring.

Table 30. The surface roughness at a fluence of $F_{1}=1.238 \mathrm{~J} / \mathrm{cm}^{2}$ per pulse

\begin{tabular}{|c|c|c|l|}
\hline $\begin{array}{c}\text { Raster speed } \\
{[\mathrm{mm} / \mathrm{s}]}\end{array}$ & $\begin{array}{c}\mathrm{R}_{\mathrm{a}} \\
{[\mathrm{nm}]}\end{array}$ & $\begin{array}{c}\mathrm{R}_{\mathrm{q}} \\
{[\mathrm{nm}]}\end{array}$ & $\begin{array}{c}\mathrm{R}_{\mathrm{t}} \\
{[\mu \mathrm{m}]}\end{array}$ \\
\hline$* 0$ & 311.9 & 394 & 4.98 \\
\hline$* 0$ & 282.5 & 358.9 & 4.87 \\
\hline$* 0$ & 389.8 & 471.9 & 6.2 \\
\hline 4 & 365.8 & 451.3 & 5.2 \\
\hline 6 & 500.8 & 623.9 & 5.18 \\
\hline 8 & 470.3 & 574.8 & 6.2 \\
\hline 10 & 367 & 452.9 & 4.1 \\
\hline
\end{tabular}

*as-received, without any laser processing- first two data points from (Sabau, Jun, and McClurg 2020).

\subsubsection{Chemical analysis of water used in salt spray tests and evolution of corrosion damage}

The composition of the water used in the salt spray tests is shown in Table 31 .

Table 31. Chemical analysis on the tap water used in salt spray tests

\begin{tabular}{|l|c|}
\hline Properties/concentration of chemical species & Values \\
\hline $\mathrm{pH}$ & 7.93 \\
\hline Conductivity, $\mu \mathrm{mho}$ & 267 \\
\hline Calcium $\mathrm{Hardness}$, as $\mathrm{CaCO}_{3}, \mathrm{mg} / \mathrm{L}$ & 79 \\
\hline Iron, as $\mathrm{Fe}, \mathrm{mg} / \mathrm{L}$ & 0.06 \\
\hline Copper, as $\mathrm{Cu}, \mathrm{mg} / \mathrm{L}$ & 0.01 \\
\hline Zinc, as $\mathrm{Zn}, \mathrm{mg} / \mathrm{L}$ & 0.03 \\
\hline Sodium, as $\mathrm{Na}, \mathrm{mg} / \mathrm{L}$ & 5.8 \\
\hline Potassium, as $\mathrm{K}, \mathrm{mg} / \mathrm{L}$ & 1.6 \\
\hline Chloride, as $\mathrm{Cl}, \mathrm{mg} / \mathrm{L}$ & 8.6 \\
\hline Sulfate, as $\mathrm{SO}_{4}, \mathrm{mg} / \mathrm{L}$ & 16 \\
\hline Nitrate, as $\mathrm{NO}_{3}, \mathrm{mg} / \mathrm{L}$ & 2.4 \\
\hline Phosphate, $\mathrm{as}_{4}, \mathrm{mg} / \mathrm{L}$ & $<0.1$ \\
\hline Silica, as $\mathrm{SiO}_{2}, \mathrm{mg} / \mathrm{L}$ & 5.6 \\
\hline
\end{tabular}




\subsubsection{X-ray photoelectron spectroscopy of blister regions}

Two blisters and the corresponding metal substrate below the blisters were analyzed using XPS as follows. The specimens were washed with water prior to the XPS analysis. XPS was performed with a Thermo Scientific Model K-Alpha XPS instrument, using a relatively large X-ray spot size $(400 \mu \mathrm{m})$ to obtain an average surface composition over the largest possible area(Sabau, Meyer, and Leonard 2020). First, two blisters were removed using a double-sided tape from specimen to reveal the underlying material. The blister material on the tape was mounted and analyzed. In order to identify possible causes for blister formation, the back-side surface of the blister, which was in contact with the metal surface, and its corresponding metal surface was analyzed. In the top two rows of 
Table 32, the elemental analysis of coating surface away from the blister regions after a short Ar-ion etching is presented; $\mathrm{C}, \mathrm{O}, \mathrm{Al}$, and $\mathrm{N}$ made up $>97$ at.\% with small amounts of $\mathrm{Cl}, \mathrm{Mg}, \mathrm{Si}, \mathrm{Na}, \mathrm{Ca}, \mathrm{Cu}$, and $\mathrm{Zn}$. After the Ar-ion etching for 1,600s, which would have removed $\sim 150 \mathrm{~nm}$ of the $\sim 20$ micron thick coating, $\mathrm{C}$ made up $>90$ at. $\%$ with $\mathrm{O}, \mathrm{Al}$, and $\mathrm{N}$ at $\sim 1-5$ at.\% and small amounts of $\mathrm{Cl}, \mathrm{Mg}, \mathrm{Si}, \mathrm{Na}$, $\mathrm{Ca}, \mathrm{Cu}$, and $\mathrm{Zn}$. In third and fourth data rows present the data for the surface composition of the backside of the coating in the blister 1 and 2, which are labeled as Coating B1 and B2, respectively. The backside of the coating had primarily $\mathrm{C}, \mathrm{O}$, and $\mathrm{Al}$ (all three $\sim 97$ at. \%) and small amounts of $\mathrm{N}, \mathrm{Cl}, \mathrm{Mg}, \mathrm{Na}, \mathrm{Ca}$, $\mathrm{Cu}$, and $\mathrm{Zn}$. In the last two rows, the metal substrates that were right below the blisters showed that $\mathrm{C}, \mathrm{O}$, and $\mathrm{Al}$ were most abundant (all three at $>98$ at.\%) with small amounts of $\mathrm{Cl}, \mathrm{Mg}, \mathrm{Na}, \mathrm{N}$, and $\mathrm{Cu}$. In all measured spots, no distinctive difference of elemental composition was found, indicating that this chemical analysis does not identify any obvious cause for the blister formation. 
Table 32. Surface composition (at.\%) nominal coating, back-surface of the blister coating, and metal surface below the blister area

\begin{tabular}{|c|c|c|c|c|c|c|c|c|c|c|c|c|}
\hline Material & Location & $\mathbf{C}$ & $\mathbf{O}$ & $\mathbf{A l}$ & $\mathbf{N}$ & $\mathbf{C l}$ & $\mathbf{M g}$ & $\mathbf{N a}$ & $\mathbf{C a}$ & $\mathbf{C u}$ & $\mathbf{Z n}$ & $\mathbf{S i}$ \\
\hline Coating & Top surface & 71.7 & 17.1 & 1.8 & 7.6 & 0.2 & 0.1 & 0.5 & 0.4 & 0.2 & 0.1 & 0.5 \\
\hline Coating & $\begin{array}{c}\sim 150 \mathrm{~nm} \\
\text { below top surf. }\end{array}$ & 91.4 & 4.7 & 1.1 & 1.8 & 0.2 & 0.0 & 0.1 & 0.2 & 0.1 & $\operatorname{tr}$ & 0.4 \\
\hline Coating B1 & Back-side & 40.8 & 40.5 & 17.5 & 0.4 & 0.2 & 0.2 & 0.3 & 0.1 & $\operatorname{tr}$ & 0.0 & - \\
\hline Coating B2 & Back-side & 38.9 & 41.8 & 18.1 & 0.3 & 0.1 & 0.4 & 0.2 & 0.1 & 0.1 & tr & - \\
\hline $\begin{array}{c}\text { Metal } \\
\text { (below B1) }\end{array}$ & Top surface & 40.0 & 41.4 & 18.0 & 0.0 & 0.2 & 0.2 & 0.2 & - & 0.0 & - & - \\
\hline $\begin{array}{c}\text { Metal } \\
\text { (below B2) }\end{array}$ & Top surface & 37.5 & 42.3 & 19.4 & 0.2 & 0.2 & 0.2 & 0.2 & - & 0.1 & - & - \\
\hline
\end{tabular}

Blister 1 and 2 are designated by B1 and B2, respectively. 


\section{FATIGUE TESTING NEEDED FOR DEMONSTRATION/IMPLEMENTATION}

The objective of this section is to identify the fatigue testing needs and funding resources needed to complete this testing. The fatigue testing will assess any possible reduction in fatigue life due to laserinterference structuring and provide critical additional data on fatigue life of the laser-interference treated specimens. The extensive microstructural characterization of surfaces/sub-surface coupled with lifetime performance data would provide the scientific basis for understanding the specific chemical, morphological, and microstructural changes induced by the laser-interference that affect the surface adhesion and enhance corrosion protection. This wealth of data will provide the community the basis for developing, optimizing, and transitioning these non-chemical, laser-based surface treatments.

\subsection{PROPOSED FATIGUE TESTING PLAN}

Consistent with prior Air Force efforts on single-laser beam depainting efforts, the testing will be conducted at performed at Westmoreland Testing and Research (WMTR). The testing plans considers the testing of the following types of A12024-T3 specimens:

- 3 baseline conditions: bare aluminum, sulfuric acid anodized (SAA), chromated conversion coated (CCC), and

- 5 laser-interference conditions.

The fatigue testing tasks envisioned include:

- Procurement of baseline specimens ( 1 " $\times 5$ " size),

- $\quad$ Laser structuring (ORNL),

- Machining of test specimens according to ASTM E466 (WMTR)

- $\quad$ Fatigue testing ASTM E466 (WMTR)

- 64, 128, or 192 specimens as budget allows (3 baseline conditions, 5 laser-structured conditions, 1 to 3 stress levels, 8 samples/stress level/condition)

- At each stress level the following testing paramters are considered: $\mathrm{R}=0.1$, a frequency of $30 \mathrm{~Hz}$, and a discontinue limit of 2,000,000 cycles.

- Data review, interpretation, and reporting

Three options were selected to test at one stress level, two, or three stress levels. Three quotes were obtained for WMTR for fatigue testing at one, two, or three stress levels. The WMTR quotes are based on 
estimated average runtime $=<24$ Hours; For tests slightly above the $24 \mathrm{~h}$, the costs will be billed based on actual runtime at the following rate: $\$ 145.00$ (Machine) $+\$ 216.00+\$ 9 / \mathrm{h}$ over 24 hours.

Table 33. Fatigue testing options and costs.

\begin{tabular}{|c|c|c|c|c|c|c|c|}
\hline Option & $\begin{array}{c}\text { Total } \\
\text { specimens }\end{array}$ & $\mathbf{4 2}$ ksi & $\mathbf{3 9}$ ksi & $\mathbf{4 6}$ ksi & $\begin{array}{c}* \text { WMTR } \\
\text { quote [\$K] }\end{array}$ & $\begin{array}{c}* * \text { ORNL } \\
\text { costs } \\
{[\mathbf{S K}]}\end{array}$ & $\begin{array}{c}\text { Total costs } \\
{[\mathbf{S K}]}\end{array}$ \\
\hline 1 & 64 & 0 & 64 & 0 & 27 & 25 & 52 \\
\hline 2 & 128 & 64 & 64 & 0 & 54 & 35 & 89 \\
\hline 3 & 192 & 64 & 64 & 64 & 80 & 45 & 125 \\
\hline
\end{tabular}

*Include ORNL composite overhead rate of $14.10 \%$ on subcontracts

**ORNL costs include materials, laser setup, and data review, interpretation, and reporting 


\section{CONCLUSIONS AND IMPLICATIONS FOR FUTURE RESEARCH}

In this study, the surface chemistry, sub-surface microstructural changes including those in the oxide layer, coating adhesion, and corrosion resistance of coated specimens of AA 2024-T3 aluminum alloy were investigated for a novel surface processing method using laser interferometry produced by two beams of a pulsed Nd:YAG laser. The as-received A12020-T3 specimens, with cracking, rolling marks and hydrocarbon contamination, were laser-interference structured without employing any polishing, cleaning or any other surface alteration techniques. The specimens were spray painted with a chromatecontaining epoxy primer, CA7233, compliant to MIL-PRF-23377 Type I Class C2 specification.

Aside from demonstrating outstanding corrosion protection, project objectives were met. For example, laser processed specimens meet the performance requirements in the coating adhesion specifications. On corrosion protection, our major accomplishment was to attain only very minor corrosion damage on only $33 \%$ of specimens by simply using acetone wiping right after the laser structuring. These results indicate that the laser-interference technique with the minor additional acetone wiping has the potential to be further developed as a nonchemical surface preparation technique for chromatecontaining epoxy primers coating systems. The main findings are summarized in this section, mainly for a laser-interference induced structuring with a spatial period of $\sim 1.7 \mu \mathrm{m}$. The finding implications on the use of this laser technique as a surface preparation for coating applications are discussed.

Surface chemistry changes due to laser processing were monitored using XPS. Near surface microstructural changes have been investigated with scanning electron microscopy and energy dispersive x-ray spectroscopy (SEM/EDS) and scanning transmission electron microscopy (STEM) for several numbers of laser-interference shots. The as-received and unprocessed aluminum surfaces are not smooth at all, exhibiting grooves in the horizontal direction from the rolling operation, quite few microcracks, and pinholes. In spite of the relatively rough original surface, the structuring by the laser-interference is thus shown to be pretty robust. SEM microstructure pictures of the top surface for spatial period of $\sim 1.7 \mu \mathrm{m}$ shows not only the interference-induced structuring but also blister-like features and end even micro-holes. These blister-like features and the tiny holes are likely due to the melting of the wall of the top-most sub-surface voids, likely contributing to roughness and increase in surface area. The as-received A12020-T3 specimens exhibited a uniform distribution of the voids and precipitates. The SEM micrographs also indicate the minimization of surface defects as all the sharp features from a rolling surface were smoothed by laserstructuring. The sub-surface microstructure modifications due to the laser-interference include precipitate 
dissolution. For the laser-structured Al 2024 specimens, the STEM images as well as EDS X-ray maps indicate that the laser-interference processing reduced the formation of CuMn-rich precipitates over a 500$800 \mathrm{~nm}$ depth from top surface. The precipitate dissolution is expected to lead to an increase in corrosion protection of the laser-interference treated surface as the localized corrosion would be reduced.

The XPS data shows that this laser technique is effective at removal of some surface contaminants, particularly for $\mathrm{C}$ which was modestly reduced for 2-shot processing dramatically reduced for 8-shot. Following laser processing, a new oxide grows on the much cleaner surface. The XPS data clearly shows that the thickness of the Al oxide layer is larger than that for the baseline specimen and that the oxide thickness increases with the number of shots per spot. Based on the XPS analysis, the amount of newly grown Al-oxide was found more uniformly distributed than that for the baseline specimen. The additional thicker oxide on Al alloys is likely to increase the corrosion resistance of the coated Al 2024.

The oxide thickness for three laser beam angles of $12^{\circ}$ (spatial period of $\sim 1.7 \mu \mathrm{m}$ ) $36^{\circ}$ (spatial period of $\sim 0.6 \mu \mathrm{m}$ ) and $72^{\circ}$ (theoretical periodicity of $\sim 0-.3 \mu \mathrm{m}$ was not attained due to widespread melting), was found to reach a maximum for 2 shots/spot and minimum for 8 shots/spot at the same laser beam angle of $36^{\circ}$ (periodicity of $\sim 0.6 \mu \mathrm{m}$ ). These results for three laser beam angles indicate that the beam angle would be an effective process variable for controlling the oxide thickness, as needed for various applications. Coating adhesion test results were presented for a primer that was coated on laser-interference structured surfaces of as-received A12024-T3 specimens, i.e., without employing any polishing, cleaning, or any other surface alteration techniques. The specimens were spray painted with a chromate-containing epoxy primer, CA7233, compliant to MIL-PRF-23377 Type I Class C2 specification. The adhesion of primer on Al surface with different treatments was assessed using the ASTM D3359 X-cut and cross-hatch tests. It was found that the laser processed specimens meet the performance requirements in the coating adhesion specifications by having a higher or identical ranking for coating adhesion specimens than those prepared with current state-of-the-art chemical conversion or sulfuric acid anodizing. The rastering with either $4 \mathrm{~mm} / \mathrm{s}$ or $6 \mathrm{~mm} / \mathrm{s}$ with a laser fluence of $1.78 \mathrm{~J} / \mathrm{cm}^{2}$ is recommended to attain acceptable coating adhesion, even higher than that with the anodizing surface treatment. These results indicate that laserinterference shows significant potential as a non-chemical surface preparation technique for these coatings.

After being stored in plastic cases for up to $70 \mathrm{~d}$, without any additional cleaning, the specimens were spray painted with a chromate-containing epoxy primer, CA7233, compliant to MIL-PRF-23377 Type I Class C2 specification. The corrosion behavior of laser-interference specimens was assessed 
against that of specimens prepared by chromated conversion coating and sulfuric acid anodizing treatments. After the ASTM B117 corrosion exposure, it was found that the laser processed specimens exhibited only few blisters. On one hand, most specimens prepared at a laser fluence of $1.78 \mathrm{~J} / \mathrm{cm}^{2}$, without any additional chemical cleaning, were found to develop one very small blister after only $96 \mathrm{~h}$ of exposure. However, the growth of these blisters was not significant even after 1,000 $\mathrm{h}$ of salt spray exposure. On the other hand, only a fraction of the specimens prepared at a laser fluence of $1.24 \mathrm{~J} / \mathrm{cm}^{2}$ and acetone wiped right after the laser structuring were found to develop several tiny blisters after $790 \mathrm{~h}$ and longer exposure. Overall, it was found that the corrosion damage was minimized at a laser rastering speed of $4 \mathrm{~mm} / \mathrm{s}$, a condition for which only $33 \%$ of specimens developed very minor corrosion damage. The ASTM D1654 creepage ratings, which was used to evaluate the corrosion damage along the scribe lines, were found to be at least nine for all coated panels. These results indicate that the laser-interference technique with the additional acetone wiping has the potential to be further developed as a nonchemical surface preparation technique for chromatecontaining epoxy primers coating systems.

In conclusion, the data shows that this laser technique is effective at removal of most of surface organic and inorganic contaminants without detrimentally affecting the surface while yielding beneficial precipitate dissolution, removal of native oxide, promoting oxide regrowth, increased oxide thickness, and a more uniform oxide scale than that of baseline specimens, coating adhesion ranking comparable with chemically intensive processes, and only small blister corrosion defects. These findings indicate that the proposed interference laser processing of AA2024 sheet material would be a promising candidate as a non-chemical method for preparing the surface for coating and joining, reducing the costly and environmentally unfriendly surface treatments for aerospace $\mathrm{Al}$ alloys.

A main knowledge gap is related to size of the spatial periodicity of the laser-interference. Due to funding limitations, the proof of principle was established in this study for laser-interference induced structuring with a spatial period of $\sim 1.7 \mu \mathrm{m}$. Future work should assess the use of larger spatial periodicities, which would produce deeper surface structures than that with a spatial period of $\sim 1.7 \mu \mathrm{m}$. The open research questions should aim at identifying the optimum periodicity for coating applications. Due to our groundwork, the future would not be as exhaustive but targeted at coating adhesion and corrosion testing while documenting the surface topology. All of the laser-interference processing was performed by splitting the primary beam of a Q-switched Nd:YAG pulsed nanosecond laser into two beams and focusing them to the same spot. Thus, another knowledge gap is related to exploring the use of other new, more powerful, and high-productivity $+50 \mathrm{kHz}$ lasers. Finally, the last knowledge gap is 
related to the reducing and elimination of corrosion blister defects. This wealth of data in this report will provide the community the basis for developing, optimizing, and transitioning these non-chemical, laserbased surface treatments.

\section{ACKNOWLEDGEMENT}

This research was sponsored by Strategic Environmental Research and Development Program (SERDP) managed by U.S. Department of Defense, U.S. Department of Energy, and U.S. Environmental Protection Agency. This manuscript has been authored by UT-Battelle, LLC under Contract No. DE-AC0500OR22725 with the U.S. Department of Energy. The authors acknowledge the following technical support: Dr. Michael Lance of ORNL for providing useful guidance on profilometry, Dana McClurg of ORNL for conducting the profilometry measurements; Tom Naguy, Deputy Technical Director, Air Force Materiel Command, W-P AFB; Michael Casey Jones, Materials Engineer, W-P AFB; Rick Osterman and Stan Bean of M\&P Solutions LLC, Pahrump, NV for guidance on aerospace applications. 


\section{REFERENCES}

Arenas, M. A., A. Conde, and J. J. de Damborenea. 2010. 'Effect of acid traces on hydrothermal sealing of anodising layers on 2024 aluminium alloy', Electrochimica Acta, 55: 8704-08.

Armitage, Peter, Geoffrey Berry, and John Nigel Scott Matthews. 2008. Statistical methods in medical research (John Wiley \& Sons).

B117-16, ASTM Standard. 2016. "Standard Practice for Operating Salt Spray (Fog) Apparatus." In.: ASTM International West Conshohocken, PA.

Berger, Jana, Marzellus Grosse Holthaus, Nicola Pistillo, Teja Roch, Kurosch Rezwan, and Andrés Fabián Lasagni. 2011. 'Ultraviolet laser interference patterning of hydroxyapatite surfaces', Applied Surface Science, 257: 3081-87.

Bieda, Matthias, Eckhard Beyer, and Andrés F Lasagni. 2010. 'Direct fabrication of hierarchical microstructures on metals by means of direct laser interference patterning', Journal of Engineering Materials and Technology, 132.

Boisier, Grégory, Nadine Pébère, Catherine Druez, Martine Villatte, and Stéphane Suel. 2008. 'FESEM and EIS Study of Sealed AA2024 T3 Anodized in Sulfuric Acid Electrolytes: Influence of Tartaric Acid', Journal of The Electrochemical Society, 155: C521-C29.

Brown, GM, and K Kobayashi. 2001. 'Nucleation and growth of a chromate conversion coating on aluminum alloy AA 2024-T3', Journal of The Electrochemical Society, 148: B457.

Costil, S, A Lamraoui, C Langlade, O Heintz, and R Oltra. 2014. 'Surface modifications induced by pulsedlaser texturing - Influence of laser impact on the surface properties', Applied Surface Science, 288: 542-49.

Critchlow, GW, DM Brewis, DC Emmony, and CA Cottam. 1995. 'Initial investigation into the effectiveness of CO2-laser treatment of aluminium for adhesive bonding', International Journal of Adhesion and Adhesives, 15: 233-36.

D'alessandria, M, A Lasagni, and F Mücklich. 2008. 'Direct micro-patterning of aluminum substrates via laser interference metallurgy', Applied Surface Science, 255: 3210-16.

Daniel, C., F. Mücklich, and Z. Liu. 2003. 'Periodical micro-nano-structuring of metallic surfaces by interfering laser beams', Applied Surface Science, 208-209: 317-21.

Davoodabadi, Ali, Jianlin Li, Yongfeng Liang, Robert Wang, Hui Zhou, David L Wood III, Timothy J Singler, and Congrui Jin. 2018. 'Characterization of surface free energy of composite electrodes for lithium-ion batteries', Journal of The Electrochemical Society, 165: A2493.

Funke, W. 1985. 'The role of adhesion in corrosion protection by organic coatings', Journal of the oil and colour chemists' association, 68: 229-32.

González, J. A., V. López, E. Otero, and A. Bautista. 2000. 'Postsealing Changes in Porous Aluminum Oxide Films Obtained in Sulfuric Acid Solutions', Journal of The Electrochemical Society, 147: 984-90.

Iannuzzi, M., and G. S. Frankel. 2007. 'Inhibition of Aluminum Alloy 2024 Corrosion by Vanadates: An In Situ Atomic Force Microscopy Scratching Investigation', CORROSION, 63: 672-88.

Ilevbare, G. O., and J. R. Scully. 2001a. 'Mass-Transport-Limited Oxygen Reduction Reaction on AA2024T3 and Selected Intermetallic Compounds in Chromate-Containing Solutions', CORROSION, 57: 134-52.

2001b. 'Oxygen Reduction Reaction Kinetics on Chromate Conversion Coated Al-Cu, Al-Cu-Mg, and Al-Cu-Mn-Fe Intermetallic Compounds', Journal of The Electrochemical Society, 148: B196B207.

Ilevbare, G. O., J. R. Scully, J. Yuan, and R. G. Kelly. 2000. 'Inhibition of Pitting Corrosion on Aluminum Alloy 2024-T3: Effect of Soluble Chromate Additions vs Chromate Conversion Coating', CORROSION, 56: 227-42. 
Jeong, Yong-Hoon, Han-Cheol Choe, and William A Brantley. 2011. 'Nanostructured thin film formation on femtosecond laser-textured Ti-35Nb-xZr alloy for biomedical applications', Thin Solid Films, 519: 4668-75.

Jun, Jiheon. 2016. 'Localized Corrosion of Super 13Cr Stainless Steel in 1D Pit for H2S-free and Sour Brines at An Elevated Temperature', Localized Corrosion of Super 13Cr Stainless Steel in 1D Pit for $\mathrm{H}_{2} \mathrm{~S}$-free and Sour Brines at An Elevated Temperature, $\mathrm{PhD}$ thesis, The Ohio State University.

Jun, Jiheon, K. Holguin, and G. S. Frankel. 2013. 'Pitting Corrosion of Very Clean Type 304 Stainless Steel', CORROSION, 70: 146-55.

Kai, Dou, R. L. Parkhill, J. Wu, and E. T. Knoobe. 2001. 'Surface microstructuring of aluminum alloy 2024 using femtosecond excimer laser irradiation', IEEE Journal of Selected Topics in Quantum Electronics, 7: 567-78.

Kalantary, M. R., D. R. Gabe, and D. H. Ross. 1992. 'A model for the mechanism of nickel fluoride cold sealing of anodized aluminium', Journal of Applied Electrochemistry, 22: 268-76.

Kendig, M. W., and R. G. Buchheit. 2003. 'Corrosion Inhibition of Aluminum and Aluminum Alloys by Soluble Chromates, Chromate Coatings, and Chromate-Free Coatings', CORROSION, 59: 379-400.

Laget, V., C. S. Jeffcoate, H. S. Isaacs, and R. G. Buchheit. 2003. 'Dehydration-Induced Loss of Corrosion Protection Properties in Chromate Conversion Coatings on Aluminum Alloy 2024-T3', Journal of The Electrochemical Society, 150: B425-B32.

Lasagni, A., M. D'Alessandria, R. Giovanelli, and F. Mücklich. 2007. 'Advanced design of periodical architectures in bulk metals by means of Laser Interference Metallurgy', Applied Surface Science, 254: 930-36.

Lasagni, Andres, Dimitri Benke, Tim Kunze, Matthias Bieda, Sebastian Eckhardt, Teja Roch, Denise Langheinrich, and Jana Berger. 2015. 'Bringing the Direct Laser Interference Patterning Method to Industry: a One Tool-Complete Solution for Surface Functionalization', Journal of Laser Micro/Nanoengineering, 10.

Lasagni, Andrés F, Carsten Gachot, Kim E Trinh, Michael Hans, Andreas Rosenkranz, Teja Roch, Sebastian Eckhardt, Tim Kunze, Matthias Bieda, and Denise Günther. 2017. "Direct laser interference patterning, 20 years of development: from the basics to industrial applications." In Laser-based Micro-and Nanoprocessing XI, 1009211. International Society for Optics and Photonics.

Lasagni, Andrés F, Teja Roch, Denise Langheinrich, Matthias Bieda, and Andreas Wetzig. 2011. 'Large area direct fabrication of periodic arrays using interference patterning', Physics Procedia, 12: 21420.

Li, R., M. G. S. Ferreira, A. Almeida, R. Vilar, K. G. Watkins, M. A. McMahon, and W. M. Steen. 1996. 'Localized corrosion of laser surface melted 2024-T351 aluminium alloy', Surface and Coatings Technology, 81: 290-96.

Lopez-Garrity, O., and G. S. Frankel. 2014a. 'Corrosion Inhibition of AA2024-T3 By Sodium Silicate', Electrochimica Acta, 130: 9-21.

2014b. 'Corrosion Inhibition of Aluminum Alloy 2024-T3 by Sodium Molybdate', Journal of The Electrochemical Society, 161: C95-C106.

Mansfeld, F., C. Chen, C. B. Breslin, and D. Dull. 1998. 'Sealing of Anodized Aluminum Alloys with Rare Earth Metal Salt Solutions', Journal of The Electrochemical Society, 145: 2792-98.

Marshall, George C. 1963. "Optical Properties of Satellite Materials-The Theory of Optical and Infrared Properties of Metals." In.: National Aeronautics and Space Administration Washington DC.

McCafferty, E., P. G. Moore, and G. T. Peace. 1982. 'Effect of Laser-Surface Melting on the Electrochemical Behavior of an Al-1\% Mn Alloy', Journal of The Electrochemical Society, 129: 917.

McDaniel, Clare, Olga Gladkovskaya, Aiden Flanagan, Yury Rochev, and Gerard M O'Connor. 2015. 'In vitro study on the response of RAW264. 7 and MS-5 fibroblast cells on laser-induced periodic surface structures for stainless steel alloys', RSC Advances, 5: 42548-58. 
McMahon, M. A., K. G. Watkins, W. M. Steen, Z. Liu, R. Vilar, and M. G. S. Ferreira. 1994. 'Laser surface alloying of 2014 aluminium alloy for enhanced corrosion resistance', International Congress on Applications of Lasers \& Electro-Optics, 1994: 135-44.

Meyer, Harry M., Adrian S. Sabau, and Claus Daniel. 2019. 'Surface chemistry and composition-induced variation of laser interference-based surface treatment of Al alloys', Applied Surface Science, 489: 893-904.

Mücklich, F., A. Lasagni, and C. Daniel. 2005. 'Laser interference metallurgy—periodic surface patterning and formation of intermetallics', Intermetallics, 13: 437-42.

Nardeli, J. V., D. V. Snihirova, C. S. Fugivara, M. F. Montemor, E. R. P. Pinto, Y. Messaddecq, and A. V. Benedetti. 2016. 'Localised corrosion assessement of crambe-oil-based polyurethane coatings applied on the ASTM 1200 aluminum alloy', Corrosion Science, 111: 422-35.

Nardeli, Jéssica V., Cecilio S. Fugivara, Maryna Taryba, M. F. Montemor, Sidney J. L. Ribeiro, and Assis V. Benedetti. 2020. 'Novel healing coatings based on natural-derived polyurethane modified with tannins for corrosion protection of AA2024-T3', Corrosion Science, 162: 108213.

Nguyen, Anh Son, and Nadine Pébère. 2016. 'A local electrochemical impedance study of the self-healing properties of waterborne coatings on 2024 aluminium alloy', Electrochimica Acta, 222: 1806-17.

Owens, Daniel K, and RC Wendt. 1969. 'Estimation of the surface free energy of polymers', Journal of applied polymer science, 13: 1741-47.

Ralston, Kevin Douglas, Tanya L Young, and Rudi G Buchheit. 2009. 'Electrochemical evaluation of constituent intermetallics in aluminum alloy 2024-T3 exposed to aqueous vanadate inhibitors', Journal of The Electrochemical Society, 156: C135.

Renshaw, T. A. 1961. 'A Study of Pore Structures on Anodized Aluminum', Journal of The Electrochemical Society, 108: 185.

Ryan, P, and PB Prangnell. 2008. 'Grain structure and homogeneity of pulsed laser treated surfaces on Alaerospace alloys and FSWs', Materials Science and Engineering: A, 479: 65-75.

Sabau, A. S., H. M. Meyer, and D. N. Leonard. 2020. 'Laser-interference Purse Number Dependence of Surface Chemistry and Sub-surface Microstructure of AA2024-T3 Alloy', Optics and Laser Technology, 102.

Sabau, Adrian S, Jiheon Jun, and Dana McClurg. 2020. 'Coating adhesion of a chromate-containing epoxy primer on A12024-T3 surface processed by laser-interference', International Journal of Adhesion and Adhesives, 102: 102641.

Sabau, Adrian S, Charles David Warren, DONALD L ERDMAN III, Claus Daniel, Timothy Skszek, and Mary M Caruso-Dailey. 2016. "A Laser Interference-Based Surface Treatment of Al and Carbon Fiber Polymer Composites for Enhanced Bonding." In.: Oak Ridge National Lab.(ORNL), Oak Ridge, TN (United States).

Sabau, Adrian S, Lang Yuan, Narendran Raghavan, Matthew Bement, Srdjan Simunovic, John A Turner, and Vipul K Gupta. 2020. 'Fluid dynamics effects on microstructure prediction in single-laser tracks for additive manufacturing of IN625', Metallurgical and Materials Transactions. B, Process Metallurgy and Materials Processing Science, 51.

Sabau, Adrian S., Clayton M. Greer, Jian Chen, Charles D. Warren, and Claus Daniel. 2016. 'Surface Characterization of Carbon Fiber Polymer Composites and Aluminum Alloys After Laser Interference Structuring', JOM, 68: 1882-89.

Scully, J. R., and Marta A. Jakab. 2019. 'Design of Cathodic Inhibitors for AA2024-T3 Guided by Understanding Heterogeneous Cathodic Reaction Kinetics', ECS Transactions, 1: 47-63.

Sehgal, A., G. S. Frankel, B. Zoofan, and S. Rokhlin. 2000. 'Pit Growth Study in Al Alloys by the Foil Penetration Technique', Journal of The Electrochemical Society, 147: 140-48.

Sehgal, A., D. Lu, and G. S. Frankel. 1998. 'Pitting in Aluminum Thin Films: Supersaturation and Effects of Dichromate Ions', Journal of The Electrochemical Society, 145: 2834-40.

Seong, Jinwook, Fan Yang, Frank Scheltens, G. S. Frankel, and N. Sridhar. 2015. 'Influence of the Altered Surface Layer on the Corrosion of AA5083', Journal of The Electrochemical Society, 162: C209C18. 
Thompson, G. E., L. Zhang, C. J. E. Smith, and P. Skeldon. 1999. 'Boric/Sulfuric Acid Anodizing of Aluminum Alloys 2024 and 7075: Film Growth and Corrosion Resistance', CORROSION, 55: 1052-61.

Veith, M., K. Andres, C. Petersen, C. Daniel, C. Holzapfel, and F. Mücklich. 2005. 'Periodical MicroStructuring of Hydride Containing Metastable Aluminumoxide using Laser Interference Metallurgy', Advanced Engineering Materials, 7: 27-30.

Walters, Craig T. 1997. 'Laser-based cleaning processes for solvent replacement', Evolving technologies for the competitive edge: 247-55.

Wang, Shan-Shan, G. S. Frankel, Jian-Tang Jiang, Jun-Feng Chen, Sheng-Long Dai, and Liang Zhen. 2013. 'Mechanism of Localized Breakdown of 7000 Series Aluminum Alloys', Journal of The Electrochemical Society, 160: C493-C502.

Wang, Shan-Shan, I. Wen Huang, Li Yang, Jian-Tang Jiang, Jun-Feng Chen, Sheng-Long Dai, David N. Seidman, G. S. Frankel, and Liang Zhen. 2015. 'Effect of Cu Content and Aging Conditions on Pitting Corrosion Damage of 7xxx Series Aluminum Alloys', Journal of The Electrochemical Society, 162: C150-C60.

Wang, Shan-Shan, Jian-Tang Jiang, Sheng-Long Dai, D. N. Seidman, G. S. Frankel, and Liang Zhen. 2014. 'Effect of Surface Roughness on Breakdown Behavior of Al-Zn-Mg-Cu Alloy', Journal of The Electrochemical Society, 161: C433-C40.

Wang, Shan-Shan, Fan Yang, and GS Frankel. 2017. 'Effect of altered surface layer on localized corrosion of aluminum alloy 2024', Journal of The Electrochemical Society, 164: C317.

Watkins, K. G., Z. Liu, M. McMahon, R. Vilar, and M. G. S. Ferreira. 1998. 'Influence of the overlapped area on the corrosion behaviour of laser treated aluminium alloys', Materials Science and Engineering: A, 252: 292-300.

Watkins, K. G., M. A. McMahon, and W. M. Steen. 1997. 'Microstructure and corrosion properties of laser surface processed aluminium alloys: a review', Materials Science and Engineering: A, 231: 55-61.

Wenzel, Robert N. 1936. 'Resistance of solid surfaces to wetting by water', Industrial \& Engineering Chemistry, 28: 988-94.

Yu, Q. S., C. M. Reddy, C. E. Moffitt, D. M. Wieliczka, R. Johnson, J. E. Deffeyes, and H. K. Yasuda. 2000. 'Improved Corrosion Protection of Aluminum Alloys by System Approach Interface Engineering: Part 2-AA2024-T3', CORROSION, 56: 887-900.

Zhao, J., L. Xia, A. Sehgal, D. Lu, R. L. McCreery, and G. S. Frankel. 2001. 'Effects of chromate and chromate conversion coatings on corrosion of aluminum alloy 2024-T3', Surface and Coatings Technology, 140: 51-57. 


\section{APPENDIX A. LIST OF SCIENTIFIC/TECHNICAL PUBLICATIONS}

The following scientific and technical publications were produced during the course of the project:

1. J. Jun, A.S. Sabau, and M.S. Stephens, Corrosion Behavior of Laser Interference Structured Al 2024 with Chromate-containing Epoxy Primer Coatings, CORROSION, 2021, Vol. 77 (5): pp. 577-590. doi: https://doi.org/10.5006/3717.

2. Chen, J., Sabau, A.S., H.M. Meyer III, and D.N., Leonard, Surface and subsurface characterization of laser-interference structured Ti6A14V, Applied Surface Science, Vol. 555, 149576, doi: 10.1016/j.apsusc.2021.149576, 2021.

3. A.S. Sabau, H.M. Meyer III, and D.N., Leonard, Laser-interference Pulse Number Dependence of Surface Chemistry and Sub-surface Microstructure of AA2024-T3 Alloy, Optics and Laser Tech., Vol. 131, Nov. 2020, doi:10.1016/j.optlastec.2020.106457

4. A.S. Sabau, J. Jun, D. McClurg, Coating adhesion of a chromate-containing epoxy primer on A12024-T3 surface processed by laser-interference, Int. J. of Adhesion and Adhesives, Vol. 102, 2020: p. 102641. doi:10.1016/j.ijadhadh.2020.102641

5. H.M. Meyer III, A.S. Sabau, and C. Daniel, Surface chemistry and composition-induced variation of laser interference-based surface treatment of Al alloys, Applied Surface Science, September 2019, Vol. 489, pp. 893-904.

6. A.S. Sabau, J. Jun, Z. Burns and M. Stephens, Corrosion Resistance of Laser-Interference Structured Aluminum alloy 2024 Coated with MIL-PRF-85582 Primer, Paper no: 0218_0314_000108, 2019 DoD-Allied Nations Technical Corrosion Conference: Corrosion Prevention and Control, Oklahoma City, OK, Aug. 11-15, 2019.

7. J. Jun, AS Sabau, Z Burns and M. Stephens, Corrosion Performance of MIL PRF- 23377 Primer on Laser-Interference Structured Aluminum alloy 2024, Paper no: 0218_0314_000109, 2019 DoD-Allied Nations Technical Corrosion Conference: Corrosion Prevention and Control, Oklahoma City, OK, Aug. 11-15, 2019.

The following webinar was presented with the proposed laser-interference technique:

T. Naguy and A.S. Sabau, (invited) "Laser De-Paint and Surface Preparation Mechanism Technologies." Sabau presented the 2-nd part "Laser-Interference Surface Preparation for Enhanced Coating Adhesion and Adhesive Joining of Multi-Materials." Presentation was part of the SERDP \& ESTCP Webinar Series, October 19, 2017, https://www.serdp-estcp.org/Tools-andTraining/Webinar-Series/10-19-2017 
The following abstracts for conference or symposium presentations (i.e., without a companion paper) were written during the course of the project:

Adrian S. Sabau, Harry M. Meyer III, Donovan N. Leonard, and Jian Chen, (invited) Sub-Surface Microstructure of Aluminum Alloys after Laser-Interference Structuring, International Conference on Processing \& Manufacturing of Advanced Materials - Processing, Fabrication, Properties, Applications; THERMEC 2018, Paris, France, July 9-13, 2018.

The following patent was granted during the course of this project:

- A.S. Sabau, J. Li, H. Meyer III, C. Daniel, Laser-Interference Surface Preparation for Enhanced Coating Adhesion, U.S. Patent No. 11,065,719 07/20/2021. 